Facts and Figures

\author{
on Afghanistan
}

Input to the Integrated Computerised Data Base

UNOCHA,ISAMABAD. 
PART III

Facts and Figures

on Afghanistan

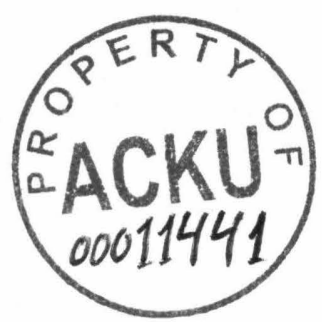

Input to the Integrated Computerised Data Base

UNOCHA,ISAMABAD. 


\begin{tabular}{|c|c|c|c|c|}
\hline $\bar{\star}$ & AFG & DATABASE & & $\begin{array}{c}\star \\
\star\end{array}$ \\
\hline ^ & & 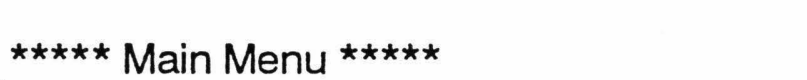 & 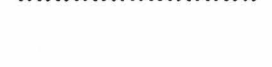 & * \\
\hline & & & & * \\
\hline * & I. & Summary Tables & & * \\
\hline * & II. & National Accounts and Production & & * \\
\hline * & III. & Public Finance & & * \\
\hline * & IV. & Monetary and Financial Statistics & & * \\
\hline * & V. & External Sector & & * \\
\hline * & vi. & Labor Force and Population & & * \\
\hline * & VII. & Infrastructure and Natural Resources & & * \\
\hline * & $\mathrm{X}$. & EXIT & & * \\
\hline * & & & & * \\
\hline * & & & & * \\
\hline * & & & & * \\
\hline & & & & * \\
\hline & & & & * \\
\hline
\end{tabular}

\section{SUMMARY TABLES}

1. Selected Macroeconomic Aggregates

2. Growth of Selected Macroeconomic Indicators

3. Basic IMF Macroeconomic Series

0. RETURN TO MAIN MENU 
Table A 1-1. Selected Macroeconomic Indicators, 1978/79-86/87

(fiscal years, beginning March 21)

\begin{tabular}{|c|c|c|c|c|c|c|c|c|c|c|c|}
\hline \multirow[b]{2}{*}{ National Accounts (constant 78/79 prices) } & \multirow[t]{2}{*}{$1978 / 79$} & \multirow[t]{2}{*}{$1979 / 80$} & \multirow[t]{2}{*}{$1980 / 81$} & \multirow[t]{2}{*}{$1981 / 82$} & \multirow[t]{2}{*}{$1982 / 83$} & \multirow[t]{2}{*}{$1983 / 84$} & \multirow[t]{2}{*}{$1984 / 85$} & \multirow[t]{2}{*}{$1985 / 86$} & \multicolumn{2}{|c|}{$\begin{array}{r}\text { Annual Avg. } \\
\text { Change (\%) } \\
1986 / 8778 / 79-86 / 87\end{array}$} & \multirow[t]{2}{*}{$\begin{array}{c}\text { Total } \\
\text { Change (\%) } \\
78 / 79-86 / 87\end{array}$} \\
\hline & & & & & & & & & & & \\
\hline GDP (unadjusted, millions of afs.) [a] & 143,500 & 139,900 & 134,700 & 138,800 & 147,300 & 150,300 & 155,600 & 152,900 & 156,300 & 1.1 & 9.0 \\
\hline GDP (adjusted UN data, millions of afs.) [b] & 117,000 & 105,000 & 100,000 & 103,000 & 104,000 & 103,000 & 107,000 & 106,000 & 108,000 & -1.0 & -8.0 \\
\hline GDP (adjusted UN data, APC) & 7.3 & -10.3 & -4.8 & 3.0 & 1.0 & -1.0 & 3.9 & -0.9 & 1.9 & -1.0 & -8.0 \\
\hline Ag., Livestock \& For. (adjusted UN data; $\%$ share of GDP) [b] & 52.8 & -- & -- & -- & -- & -- & 47.2 & 46.9 & 45.5 & -- & -- \\
\hline Ag., Livestock \& Forestry (adjusted UN data, APC) [b] & 6.1 & 2.8 & -0.8 & 3.1 & 0.0 & -3.0 & -3.0 & -2.4 & -1.1 & -2.6 & -21.0 \\
\hline Ind., Mining \& Energy (adjusted UN data; $\%$ share of GDP) [b] & 12.3 & -- & -- & -- & -- & -- & 12.5 & 13.6 & 13.6 & -- & -- \\
\hline Industry, Mining \& Energy (adjusted UN data, APC) [b] & 9.2 & -7.5 & -6.2 & -2.1 & 2.1 & -1.1 & -1.1 & 6.7 & 2.3 & 0.2 & 2.0 \\
\hline $\begin{array}{l}\text { GDP (adjusted RRNA series based on UNDP } \\
\text { and Swedish Committee revisions.) [c] }\end{array}$ & 113,699 & 99,124 & 91,628 & 91,518 & 89,520 & 85,801 & 86,163 & 82,416 & 80,973 & -3.6 & -29.0 \\
\hline Ag., Livestock \& For. (based on Swedish Committee data) [d] & -- & -- & -- & -- & -- & -- & -- & -- & -- & -6.2 & -55.0 \\
\hline \multicolumn{12}{|l|}{ Public Finance } \\
\hline Total revenue (APC) & 10.8 & -4.1 & 66.6 & 14.0 & 12.2 & 3.3 & 5.3 & 14.6 & 8.1 & 22.0 & 176.0 \\
\hline Tax revenue ( $\%$ of total) & 57.4 & 45.9 & 37.7 & 30.2 & 32.1 & 40.2 & 44.9 & 41.7 & 41.7 & -- & -- \\
\hline Non-tax revenue ( $\%$ of total) & 42.6 & 54.1 & 62.3 & 69.8 & 67.9 & 59.8 & 55.1 & 58.3 & 58.3 & -- & -- \\
\hline Total expenditure (APC) & 11.8 & 14.7 & 36.1 & 16.0 & 42.0 & 10.3 & 14.4 & 18.5 & 20.1 & 26.4 & 211.0 \\
\hline Ordinary or recurrent expenditure ( $\%$ of total) & 46.9 & 67.3 & 63.4 & 65.6 & 56.3 & 60.5 & 77.1 & 76.6 & 77.5 & -- & -- \\
\hline Development expenditure ( $\%$ of total) & 53.1 & 32.7 & 36.6 & 34.4 & 43.7 & 39.5 & 22.9 & 23.4 & 22.5 & -- & -- \\
\hline $\begin{array}{l}\text { Overall central government deficit } \\
\text { (as } \% \text { of total expenditures) }\end{array}$ & 17.0 & 28.1 & 16.4 & 13.7 & 33.7 & 37.0 & 36.6 & 38.7 & 44.8 & -- & -- \\
\hline
\end{tabular}

Note: APC = Annual Percentage Change.

a] Conversion by the UN of the official NMP series into GDP on the basis of GOA responses to UN questionnaires, without adjusting the official figures to reflect the depressed economic conditions.

b] Above GDP series, adjusted by the UN to reflect the economic deterioration not shown in the official figures.

c] Adjusted UNGDP data revised to reflect the reduction in agricultural production. The absolute figures for agricultural production were derived based on the sector's proportion of GDP, as shown in Table All-2. These figures were then revised downward based on the reduction in agricultural production shown under the

[d] These adjustments, based on data from the First Swedish Committee for Afghanistan Report of May 1988, show a larger drop in agricultural production than the UN adjusted data.

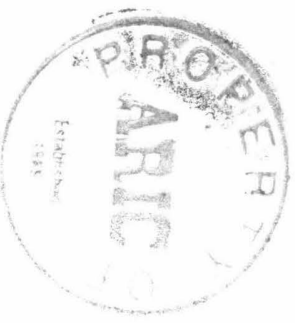


Table A I-1 (continued). Selected Macroeconomic Indicators, 1978/79-86/87
(fiscal years, beginning March 21)

\begin{tabular}{|c|c|c|c|c|c|c|c|c|c|c|c|}
\hline & $1978 / 79$ & $1979 / 80$ & $1980 / 81$ & $1981 / 82$ & $1982 / 83$ & $1983 / 84$ & $1984 / 85$ & $1985 / 86$ & $1986 / 87$ & $\begin{array}{r}\text { Annual Avg. } \\
\text { Change }(\%) \\
78 / 79-86 / 87\end{array}$ & $\begin{array}{c}\text { Total } \\
\text { Change (\%) } \\
78 / 79-86 / 87\end{array}$ \\
\hline \multicolumn{12}{|l|}{ Monetary Survey } \\
\hline Net foreign assets (APC) & 20.6 & 9.0 & 79.6 & 14.2 & -13.9 & -- & -- & -13.7 & -21.1 & -1.4 & -11.0 \\
\hline Net domestic credit (NDC, APC) [e] & 24.8 & 27.1 & -29.2 & 35.9 & 23.2 & -- & -- & 24.6 & 25.1 & 44.1 & 353.0 \\
\hline Credit to central gov't ( $\%$ of NDC) [e] & 70.5 & 65.8 & 53.5 & 57.7 & 55.1 & -- & 65.3 & 67.0 & 66.8 & -- & \\
\hline Credit to official entities (\% of NDC) & -- & -- & 0.0 & -- & 24.8 & -- & 25.1 & 22.8 & 22.5 & -- & \\
\hline Credit to private sector ( $\%$ of NDC) [e] & 29.5 & 33.6 & 46.5 & 35.8 & 16.9 & -- & 7.2 & 8.1 & 8.4 & -- & \\
\hline Money (index 1985/86 = 100) & 36.1 & 42.4 & 52.1 & 58.5 & 68.7 & 78.3 & 89.5 & 100.0 & 111.8 & -- & \\
\hline Money (as $\%$ of total liquidity) & 85.6 & 86.2 & 85.8 & 83.9 & 82.2 & 81.8 & 83.6 & 84.2 & 83.6 & -- & \\
\hline Quasi-money (as $\%$ of total liquidity) & 14.4 & 13.8 & 14.2 & 16.1 & 17.8 & 18.2 & 16.4 & 15.8 & 16.4 & -- & \\
\hline \multicolumn{12}{|l|}{ Prices \& Exchange Rates } \\
\hline Kabul Cońsumer Price Index (APC) & 5.2 & 9.9 & 12.6 & 21.5 & 16.6 & 19.6 & 27.4 & 9.7 & 12.2 & 13.7 & 109.0 \\
\hline Official exchange rate, annual avg. (afs. per U.S. dollar) & 45.00 & 42.25 & 45.85 & 50.20 & 50.60 & 50.60 & 50.60 & 50.60 & 50.60 & -- & \\
\hline Bazaar exchange rate, annual avg. (afs. per U.S. dollar) & 38.80 & 42.93 & 46.13 & 56.62 & 76.75 & 99.31 & 122.01 & 137.10 & 148.20 & -- & \\
\hline \multicolumn{12}{|l|}{ Balance of Payments } \\
\hline Exports, FOB, customs valuation (APC) [f] & 4.8 & 47.8 & 41.4 & 3.6 & 1.9 & 3.0 & -13.1 & -12.0 & -0.9 & 9.0 & 72.0 \\
\hline Imports, CIF, customs valuation (APC) [f] & 20.4 & 7.8 & 97.6 & 5.4 & 8.5 & 10.7 & 30.6 & -14.1 & 17.5 & 31.9 & 255.0 \\
\hline Overall balance (millions of U.S. dollars) & 98.2 & 59.3 & 194.5 & -32.9 & -70.3 & -215.3 & -178.6 & 3.9 & -247.0 & -- & \\
\hline Int'I reserves minus gold (in months of imports) & 11.9 & 12.4 & 5.3 & 3.7 & 3.2 & 2.4 & 2.0 & 3.0 & 2.2 & -- & \\
\hline $\begin{array}{l}\text { External public debt, disbursement basis } \\
\text { (millions of U.S. dollars) }\end{array}$ & 1,367 & 1,627 & 1,927 & 2,091 & 2,411 & -- & 2,576 & 2,743 & -1 & -- & \\
\hline Debt service ratio ( $\%$ of exports of goods \& services) & 14.4 & 14.4 & 6.5 & 14.9 & 15.6 & 16.7 & 17.8 & 10.9 & 16.0 & -- & \\
\hline \multicolumn{12}{|l|}{ Population (000) } \\
\hline Official & 13,051 & -- & -- & -- & -- & 17,672 & 18,136 & 18,614 & - & -- & \\
\hline RRNA Revised Estimates [ $\mathrm{g}$ ] & & 12,925 & 11,942 & 10,941 & 10,683 & 10,419 & 10,130 & 9,835 & 9,683 & 9,528 & \\
\hline
\end{tabular}

[e] Partial figures do not add up to totals because of rounding off and because of inadequate information about unclassified items, items in transit and float. For

the same reason, percentage shares do not add up to 100

[f] These figures differ from those in Table A $1-3$. The latter figures are reported on balance of payments basis and include adjustments in coverage, valuation and

(g) See Table A VI-3 for the derivation of these figures.

Sources: 1986 Consultations, Recent Economic Developments, IMF; Key Indicators, ADB; International Financial Statistics, IMF; "The Agricultural Survey of

Afghanistan", Swedish Committee for Afghanistan, May 1988; UNDP Discussion Paper on Afghanistan of April 1988; Yearbook of National Account Statistics, UN. 
Table A I-2. Selected Macro Financial Aggregates, 1965-88

(millions of afghanis, unless otherwise indicated)

\begin{tabular}{|c|c|c|c|c|c|c|c|c|c|c|c|c|}
\hline & 1965 & 1966 & 1967 & 1968 & 1969 & 1970 & 1971 & 1972 & 1973 & 1974 & 1975 & 1976 \\
\hline $\begin{array}{l}\text { Market exchange rate, afghanis per U.S. } \\
\text { dollar (line rf, period average) [a] }\end{array}$ & 45.00 & 45.00 & 45.00 & 45.00 & 45.00 & 45.00 & 45.00 & 45.00 & 45.00 & 45.00 & 45.00 & 45.00 \\
\hline \multicolumn{13}{|l|}{$\begin{array}{l}\text { International Reserves (millions of U.S. } \\
\text { dollars) }\end{array}$} \\
\hline Total reserves & NA & NA & NA & NA & 39.19 & 43.54 & 55.96 & 56.20 & 60.96 & 66.99 & 125.90 & 170.27 \\
\hline Total reserves minus gold (line $11 \mathrm{~d}$ ) & 8.98 & 12.10 & 5.30 & 6.12 & 8.18 & 12.53 & 26.95 & 20.80 & 21.61 & 27.64 & 86.55 & 130.92 \\
\hline Gold, national valuation (line 1and) & NA & NA & NA & NA & 31.01 & 31.01 & 29.01 & 35.40 & 39.35 & 39.35 & 39.35 & 39.35 \\
\hline \multicolumn{13}{|l|}{ Monetary Authorities } \\
\hline Foreign assets (line 11) & 2,240 & 2,256 & 1,934 & 1,689 & 1,830 & 2,148 & 2,722 & 2,821 & 3,185 & 3,550 & 6,848 & 9,350 \\
\hline Minus foreign liabilities (line 16c) & 452 & 741 & 571 & 777 & 908 & 751 & 772 & 501 & 434 & 315 & 592 & 234 \\
\hline Net domestic credit $[b]$ & 5,376 & 5,273 & 5,845 & 7,396 & 7,913 & 8,892 & 9,690 & 11,232 & 11,446 & 13,430 & 13,752 & 16,336 \\
\hline Claims on cert. govt. (line 12a) & 5,476 & 5,736 & 6,082 & 7,486 & 7,614 & 8,363 & 9,859 & 11,657 & 11,355 & 12,270 & 13,204 & 15,466 \\
\hline Claims on local government (line 12b) & 119 & 88 & 142 & 260 & 177 & 287 & 129 & 9 & 11 & 770 & 1,313 & 400 \\
\hline Claims on nonfin. pub. enter. (line 12c) & NA & NA & NA & NA & NA & NA & NA & NA & NA & NA & NA & NA \\
\hline Claims on private sector (line 12d) & 485 & 628 & 517 & 662 & 1,097 & 1,020 & 833 & 1,186 & 1,261 & 1,706 & 2,273 & 3,678 \\
\hline Claims on commercial banks (line 12e) & 70 & 130 & 174 & 109 & 130 & 658 & 266 & 367 & 408 & 1,153 & 825 & 415 \\
\hline Claims on other fin. insts. (line 12f) & NA & NA & NA & NA & NA & NA & NA & NA & NA & NA & NA & NA \\
\hline Minus cent. gov't. dep. (line 16d) & 774 & 1,309 & 1,070 & 1,121 & 1,105 & 1,436 & 1,397 & 1,987 & 1,589 & 2,469 & 3,863 & 3,623 \\
\hline Currency outside banks (line 14a) $[c]$ & 4,797 & 4,722 & 4,614 & 5,111 & 5,769 & 6,540 & 6,435 & 7,518 & 8,979 & 9,693 & $(11,427)$ & 14,225 \\
\hline Private sector deposits (line 14d) [c] & 992 & 1,158 & 1,128 & 1,792 & 1,630 & 1,419 & 2,297 & 2,134 & 1,616 & 2,019 & $(2,494)$ & 3,002 \\
\hline \multicolumn{13}{|l|}{ Time, savings, and foreign currency } \\
\hline deposits (line 15) & 297 & 253 & 295 & 263 & 589 & 680 & 681 & 811 & 1,254 & 1,748 & 1,445 & 2,306 \\
\hline Import deposits (line 16b) & NA & NA & NA & NA & 291 & 522 & 611 & 552 & 585 & 1,043 & 2,179 & 4,020 \\
\hline Capital accounts (line 17a) & 813 & 841 & 689 & 692 & 787 & 985 & 1,325 & 1,510 & 1,579 & 1,512 & 1,627 & 1,702 \\
\hline Other items, net (line 17r) & 58 & (338) & 190 & 174 & (635) & $(657)$ & (488) & 344 & $(109)$ & $(44)$ & 214 & (342) \\
\hline \multicolumn{13}{|l|}{ Commercial Banks } \\
\hline Currency and reserve deposits (line 20) & 150 & 117 & 212 & 229 & 321 & 509 & 519 & 604 & 308 & 539 & 462 & 520 \\
\hline Net foreign ass ets & NA & NA & NA & NA & NA & NA & NA & NA & NA & NA & NA & NA \\
\hline Foreign assets (line 21) & 93 & 126 & 135 & 165 & 222 & 40 & 339 & 231 & 282 & 380 & 492 & 467 \\
\hline Minus foreign liabilties (line 26c) & NA & NA & NA & NA & NA & NA & NA & NA & NA & NA & NA & NA \\
\hline Net domestic credit & 1,651 & 1,682 & 1,797 & 1,662 & 1,916 & 2,478 & 2,247 & 2,691 & 2,904 & 3,404 & 2,623 & 2,559 \\
\hline
\end{tabular}

Note: Line numbers refer to those in the country pages of IMF's International Financial Statistics.

[a] Official exchange rate.

[b] Totals do not take into accourt the NA entries.

[c] Partial figures do not add up to totals because other (minor) components of reserve money are not published in IFS 
Table A I-2 (continued). Selected Macro Financial Aggregates, 1965-88

(millions of at

\begin{tabular}{|c|c|c|c|c|c|c|c|c|c|c|c|c|}
\hline & 1965 & 1966 & 1967 & 1968 & 1969 & 1970 & 1971 & 1972 & 1973 & 1974 & 1975 & 1976 \\
\hline Claims on nonfin. pub. enter. (line 22c) & NA & NA & NA & NA & NA & NA & NA & NA & NA & NA & NA & NA \\
\hline Claims on private sector (line 22d) & 1,767 & 1,767 & 1,932 & 1,826 & 1,992 & 2,563 & 2,326 & 2,795 & 3,020 & 3,509 & 2,743 & 2,565 \\
\hline Claims on other fin. insts. (line 22f) & NA & NA & NA & NA & NA & NA & NA & NA & NA & NA & NA & NA \\
\hline Minus cent. gov't. dep. (line 26d) & 116 & 85 & 135 & 164 & 76 & 85 & 79 & 104 & 116 & 105 & 120 & 6 \\
\hline Demand deposits (line 24) & 315 & 263 & 359 & 381 & 772 & 719 & 936 & 911 & 723 & 810 & 1,054 & 1,095 \\
\hline Time, savings, and for. cur. dep. (line 25) & 654 & 665 & 639 & 667 & 825 & 973 & 1,211 & 1,541 & 1,612 & 1,825 & 1,679 & 1,851 \\
\hline Import deposits (line 26b) & NA & NA & NA & NA & NA & NA & NA & NA & NA & NA & NA & NA \\
\hline Long-term foreign liabilities (line 26cl) & NA & NA & NA & NA & NA & NA & NA & NA & NA & NA & NA & NA \\
\hline Credit from monetary authorties (line $26 \mathrm{~g}$ ) & NA & NA & NA & NA & NA & NA & NA & NA & NA & NA & NA & NA \\
\hline Capital accounts (line 27a) & 1,064 & 1,119 & 1,229 & 1,257 & 1,278 & 1,199 & 1,210 & 1,226 & 1,317 & 1,275 & 1,237 & 1,274 \\
\hline \multicolumn{13}{|l|}{.. Monetary Survey } \\
\hline Net foreign assets (line $31 \mathrm{n}$ ) & 1,881 & 1,641 & 1,498 & 1,077 & 1,143 & 1,437 & 2,288 & 2,550 & 3,032 & 3,615 & 6,748 & 9,583 \\
\hline Net domestic credit (line 32) & 6,957 & 6,825 & 7,468 & 8,949 & 9,699 & 10,712 & 11,671 & 13,556 & 13,942 & 15,682 & 15,551 & 18,480 \\
\hline Claims on cert. gov't., net (line 32an) & 4,586 & 4,342 & 4,877 & 6,201 & 6,433 & 6,842 & 8,383 & 9,565 & 9,651 & 9,697 & 9,222 & 11,837 \\
\hline Claims on local government (line $32 \mathrm{~b}$ ) & 119 & 88 & 142 & 260 & 177 & 287 & 129 & 9 & 11 & 770 & 1,313 & 400 \\
\hline Claims on nonfin. pub. enter. (line 32c) & NA & NA & NA & NA & NA & NA & NA & NA & NA & NA & NA & NA \\
\hline Claims on private sector (line $32 d$ ) & 2,252 & 2,395 & 2,449 & 2,488 & 3,089 & 3,583 & 3,159 & 3,982 & 4,280 & 5,215 & 5,016 & 6,243 \\
\hline Claims on other fin. insts. (line 321) & NA & NA & NA & NA & NA & NA & NA & NA & NA & NA & NA & NA \\
\hline Money, index 1985=100 (line 34i) & 7.60 & 7.64 & 8.68 & 9.47 & 11.05 & 12.42 & 13.08 & 14.91 & 14.95 & 16.12 & 18.90 & 21.98 \\
\hline Money, percent change (line 34x) & 12.40 & 0.44 & 13.67 & 9.05 & 16.74 & 12.40 & 5.29 & 13.96 & 0.27 & 7.83 & 17.27 & 16.28 \\
\hline Quasi-money (line 35) & 951 & 918 & 934 & 930 & 1,414 & 1,653 & 1,892 & 2,352 & 2,866 & 3,573 & 3,124 & 4,157 \\
\hline Import deposits (line 36b) & NA & NA & NA & NA & 291 & 522 & 611 & 552 & 585 & 1,043 & 2,179 & 4,020 \\
\hline Long-term foreign liabilities (line $36 \mathrm{cl}$ ) & NA & NA & NA & NA & NA & NA & NA & NA & NA & NA & NA & NA \\
\hline Other items, net (line 37r) & 1,783 & 1,405 & 1,931 & 1,813 & 965 & 1,295 & 1,790 & 2,640 & 2,204 & 2,160 & 2,022 & 1,564 \\
\hline \multirow{2}{*}{\multicolumn{13}{|c|}{ Prices }} \\
\hline & & & & & & & & & & & & \\
\hline Consumer prices, index $1985=100$ (line 64) & NA & NA & NA & NA & NA & 55.60 & 69.80 & 61.00 & 54.50 & 60.10 & 67.20 & 67.60 \\
\hline Consumer prices, percent change (line $64 x$ ) & NA & NA & NA & NA & NA & NA & 25.51 & (12.52) & $(10.68)$ & 10.23 & 11.86 & 0.60 \\
\hline
\end{tabular}


(millions of afghanis, unless otherwise indicated)

\begin{tabular}{|c|c|c|c|c|c|c|c|c|c|c|c|c|}
\hline & 1965 & 1966 & 1967 & 1968 & 1969 & 1970 & 1971 & 1972 & 1973 & 1974 & 1975 & 1976 \\
\hline \multicolumn{13}{|l|}{ Foreign trade (millions of U.S. dollars) } \\
\hline Exports, FOB (line 70d) & 73.93 & 67.36 & 66.41 & 71.82 & 81.92 & 85.57 & 99.65 & 122.47 & 143.21 & 230.00 & 217.38 & 291.32 \\
\hline Natural gas (line 70bd) & NA & NA & 1.16 & 7.74 & 12.13 & 14.50 & 15.41 & 17.76 & 17.97 & 26.29 & 46.80 & 39.58 \\
\hline Raw cotton (line $70 \mathrm{fd}$ ) & 11.13 & 11.90 & 7.86 & 5.61 & 5.60 & 8.80 & 13.25 & 11.58 & 7.24 & 31.72 & 29.79 & 64.51 \\
\hline Wool (line 70 hd) & 1.99 & 4.98 & 4.86 & 6.99 & 6.73 & 7.10 & 8.47 & 6.19 & 5.79 & 7.11 & 7.99 & 8.51 \\
\hline Carpets (line $70 \mathrm{mrd}$ ) & 8.94 & 8.00 & 5.17 & 4.52 & 6.25 & 7.20 & 8.47 & 11.23 & 14.47 & 19.90 & 17.50 & 24.90 \\
\hline Fruits and nuts (line $70 \mathrm{txd}$ ) & 24.01 & 21.81 & 25.89 & 26.91 & 28.40 & 29.00 & 28.66 & 43.78 & 68.98 & 84.42 & 69.47 & 87.66 \\
\hline Karakul skins (line 70 wld) & 16.08 & 11.72 & 14.05 & 8.32 & 13.05 & 10.60 & 18.89 & 16.39 & 16.76 & 12.18 & 10.79 & 19.77 \\
\hline Imports, CIF (line 71d) & 121.00 & 128.00 & 126.00 & 114.00 & 126.00 & 114.00 & 141.00 & 164.00 & 172.00 & 243.00 & 350.00 & 260.92 \\
\hline Imports, FOB (Line 71vd) & 105.22 & 111.30 & 109.57 & 99.13 & 109.57 & 99.13 & 122.61 & 142.61 & 149.57 & 211.30 & 304.35 & 226.89 \\
\hline Imports, commercial, CIF (line 71xd) & 56.51 & 66.68 & 62.90 & 65.57 & 72.50 & 79.00 & 84.00 & 94.89 & 120.80 & 223.05 & 231.34 & 260.86 \\
\hline
\end{tabular}


Table A I-2 (continued). Selected Macro Financial Aggregates, 1965-88

(millions of afghanis, unless otherwise indicated)

\begin{tabular}{|c|c|c|c|c|c|c|c|c|c|c|c|c|}
\hline & 1977 & 1978 & 1979 & 1980 & 1981 & 1982 & 1983 & 1984 & 1985 & 1986 & 1987 & 1988 \\
\hline \multicolumn{13}{|l|}{ Foreign trade (millions of U.S. dollars) } \\
\hline Exports, FOB (line 70d) & 306.14 & 320.69 & 474.03 & 670.23 & 694.27 & 707.62 & 728.60 & 632.90 & 556.80 & 551.90 & 511.90 & NA \\
\hline Natural gas (line 70bd) & 38.90 & 46.50 & 76.09 & 215.95 & 272.57 & $283 . \curvearrowleft 2$ & 305.30 & 314.30 & 309.40 & 259.60 & 204.50 & NA \\
\hline Raw cotton (line 7 ofd) & 40.51 & 43.47 & 41.62 & 39.78 & 22.61 & 8.95 & 10.20 & 22.40 & 19.20 & 9.80 & 8.90 & NA \\
\hline Wool (line $70 \mathrm{hd}$ ) & 5.68 & 7.37 & 13.87 & 15.22 & 23.42 & 20.53 & 25.40 & 26.10 & 22.50 & 14.60 & 3.80 & NA \\
\hline Carpets (line 70mrd) & 39.61 & 36.49 & 62.89 & 104.12 & 72.49 & 80.76 & 50.40 & 42.20 & 58.40 & 39.52 & 48.90 & NA \\
\hline Fruits and nuts (line $70 t x d$ ) & 107.82 & 124.00 & 210.40 & 216.02 & 222.07 & 153.98 & 192.00 & 181.90 & 120.00 & 134.88 & 128.60 & NA \\
\hline Karakul skins (line 70 wld) & 23.36 & 16.97 & 25.03 & 34.12 & 18.84 & 21.48 & 9.60 & 9.20 & 10.45 & 10.50 & 8.30 & NA \\
\hline Imports, CIF (line 71d) & 327.97 & 394.84 & 425.46 & 840.90 & 886.30 & 961.80 & $1,064.30$ & $1,389.50$ & $1,194.20$ & $1,403.50$ & 995.90 & NA \\
\hline Imports, commercial, CIF (line 71xd) & 328.22 & 394.84 & 315.08 & 551.08 & 651.20 & 695.20 & 846.00 & $1,097.70$ & 883.10 & 861.00 & 786.40 & NA \\
\hline
\end{tabular}


Table A I-2 (continued). Selected Macro Financial Aggregates, 1965-88

(millions of afghanis, unless otherwise indicated)

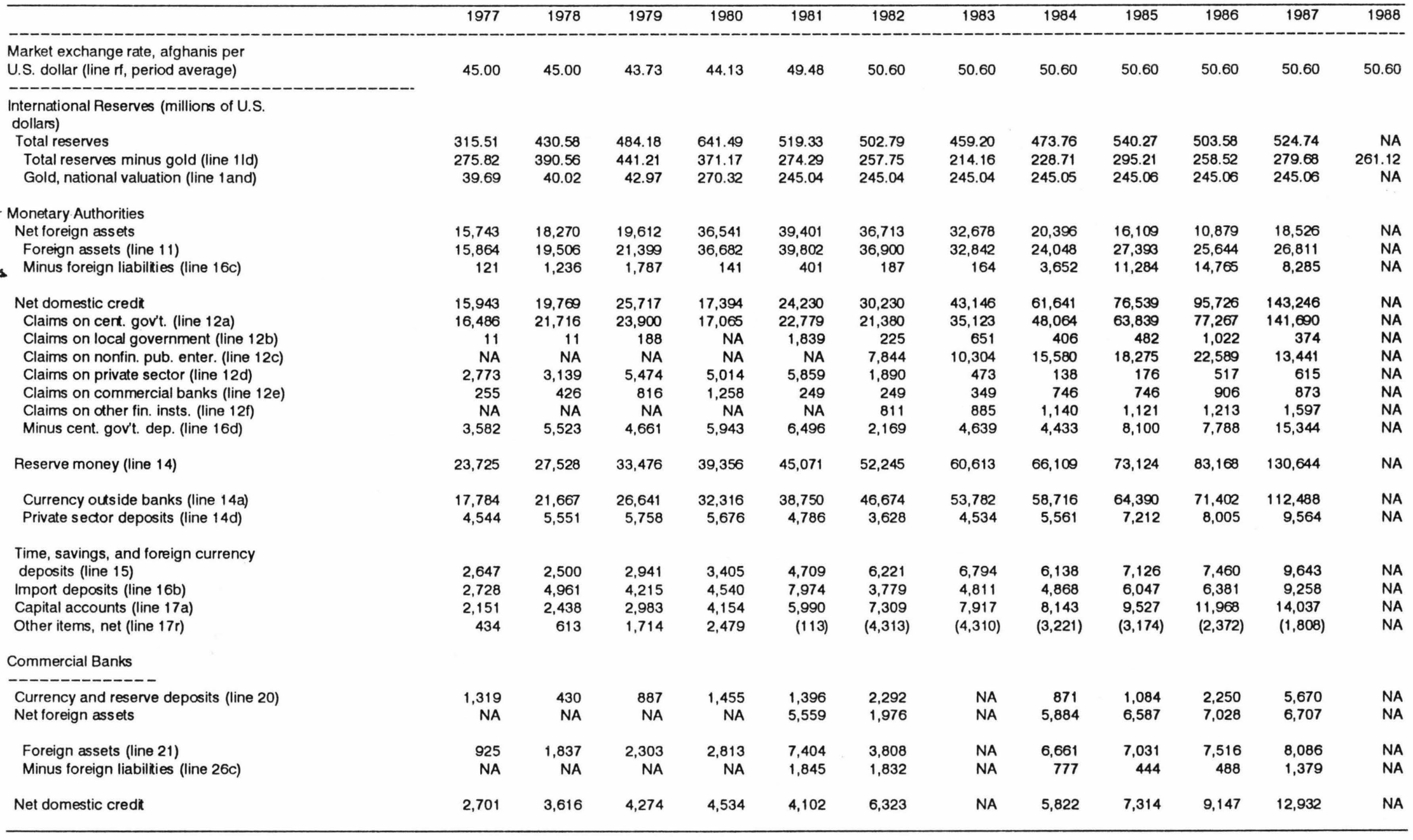


Table A I-2 (continued). Selected Macro Financial Aggregates, 1965-88 (millions of afghanis, unless otherwise indicated)

\begin{tabular}{|c|c|c|c|c|c|c|c|c|c|c|c|c|}
\hline & 1977 & 1978 & 1979 & 1980 & 1981 & 1982 & 1983 & 1984 & 1985 & 1986 & 1987 & 1988 \\
\hline Claims on nonfin. pub. enter. (line 22c) & NA & NA & NA & NA & NA & 725 & NA & 1,150 & 682 & 830 & 695 & NA \\
\hline Claims on private sector (line 22d) & 2,720 & 3,632 & 4,315 & 4,589 & 4,194 & 5,655 & NA & 4,651 & 6,552 & 8,256 & 12,083 & NA \\
\hline Claims on other fin. insts. (line 22f) & NA & NA & NA & NA & NA & 89 & NA & 76 & 106 & 92 & 239 & NA \\
\hline Minus cent. gov't. dep. (line 26d) & 19 & 16 & 41 & 55 & 92 & 146 & NA & 55 & 26 & 31 & 85 & NA \\
\hline Demand deposits (line 24) & 1,424 & 1,407 & 1,788 & 1,786 & 2,128 & 1,850 & NA & 2,946 & 3,705 & 4,127 & 5,642 & NA \\
\hline Time, savings, and for. cur. dep. (line 25) & 2,372 & 2,326 & 2,533 & 3,161 & 4,059 & 5,217 & NA & 7,339 & 7,156 & 9,218 & 12,223 & NA \\
\hline Import deposits (line 26b) & NA & NA & NA & NA & NA & (493) & NA & 1,159 & 1,998 & 2,617 & 3,836 & NA \\
\hline Long-term foreign liabilities (line 26cl) & NA & NA & NA & NA & 624 & 559 & NA & 463 & 439 & 0 & 0 & NA \\
\hline Credit from monetary authorties (line $26 \mathrm{~g}$ ) & NA & NA & NA & NA & NA & 51 & NA & 466 & 1,061 & 498 & 16 & NA \\
\hline Capital accounts (line 27a) & 1,375 & 1,587 & 1,862 & 2,079 & 2,250 & 2,106 & NA & 2,442 & 2,552 & 4,013 & $(6,122)$ & NA \\
\hline Other items, net (line 27r) & (226) & 561 & 1,281 & 1,775 & 1,997 & 1,301 & NA & $(2,238)$ & $(1,926)$ & $(2,048)$ & 9,712 & NA \\
\hline \multicolumn{13}{|l|}{ Monetary Survey } \\
\hline Net foreign assets (line $31 n$ ) & 16,667 & 20,107 & 21,915 & 39,353 & 44,960 & 38,689 & NA & 26,279 & 22,697 & 17,906 & 25,233 & NA \\
\hline Net domestic credit (line 32) & 18,390 & 22,958 & 29,176 & 20,670 & 26,428 & 34,603 & NA & 66,717 & 83,107 & 103,967 & 155,305 & NA \\
\hline Claims on cent. gov't., net (line 32an) & 12,885 & 16,177 & 19,198 & 11,067 & 16,191 & 19,065 & NA & 43,576 & 55,713 & 69,448 & 126,261 & NA \\
\hline Claims on local government (line 32b) & 11 & 11 & 188 & NA & 184 & 225 & 651 & 406 & 482 & 1,022 & 374 & NA \\
\hline Claims on nonfin. pub. enter. (line 32c) & NA & NA & NA & NA & NA & 8,569 & NA & 16,730 & 18,957 & 23,419 & 14,136 & NA \\
\hline Claims on private sector (line 32d) & 5,494 & 6,770 & 9,790 & 9,603 & 10,053 & 5,844 & NA & 4,789 & 6,728 & 8,773 & 12,698 & NA \\
\hline Claims on other fin. insts. (line 321 ) & NA & NA & NA & NA & NA & 900 & NA & 1,216 & 1,227 & 1,305 & 1,836 & NA \\
\hline Money (line 34) & 23,752 & 28,625 & 34,187 & 39,779 & 45,665 & 52,973 & 60,437 & 68,638 & 76,359 & 85,113 & 131,419 & NA \\
\hline Money, index 1985 = 100 (line 34i) & 29.70 & 36.05 & 42.37 & 52.11 & 58.47 & 68.67 & 78.32 & 89.48 & 100.00 & 111.78 & 136.52 & NA \\
\hline Money, percent change (line $34 x$ ) & 35.13 & 21.38 & 17.56 & 22.98 & 12.20 & 17.45 & 14.05 & 14.25 & 11.76 & 11.78 & 22.13 & NA \\
\hline Quasi - money (line 35) & 5,020 & 4,826 & 5,474 & 6,565 & 8,768 & 11,439 & NA & 13,476 & 14,282 & 16,678 & 21,866 & NA \\
\hline Import deposits (line $36 \mathrm{~b})$ & 2,728 & 4,961 & 4,215 & 4,540 & 7,974 & 3,286 & NA & 6,027 & 8,045 & 8,998 & 13,095 & NA \\
\hline Long-term foreign liabilities (line $36 \mathrm{cl}$ ) & NA & NA & NA & NA & 624 & 539 & NA & 463 & 439 & 0 & 0 & NA \\
\hline Other items, net (line 37r) & 3,557 & 4,653 & 7,214 & 9,139 & 10,014 & 5,036 & NA & 4,392 & 6,680 & 11,086 & 14,159 & NA \\
\hline \multicolumn{13}{|l|}{ Prices } \\
\hline & & & & & & & & & & & & \\
\hline Consumer prices, index 1985=100 (line 64) & 72.80 & 78.80 & 78.30 & 79.00 & 82.80 & 87.60 & 85.00 & 91.60 & 100.00 & NA & NA & NA \\
\hline Consumer prices, percent change (line $64 x$ ) & 7.65 & 8.22 & $(0.62)$ & 0.86 & 4.88 & 5.81 & (2.96) & 7.71 & 9.16 & NA & NA & NA \\
\hline
\end{tabular}


Table A I-3. Basic IMF Macroeconomic Series from "Recent Economic Developments" Publication

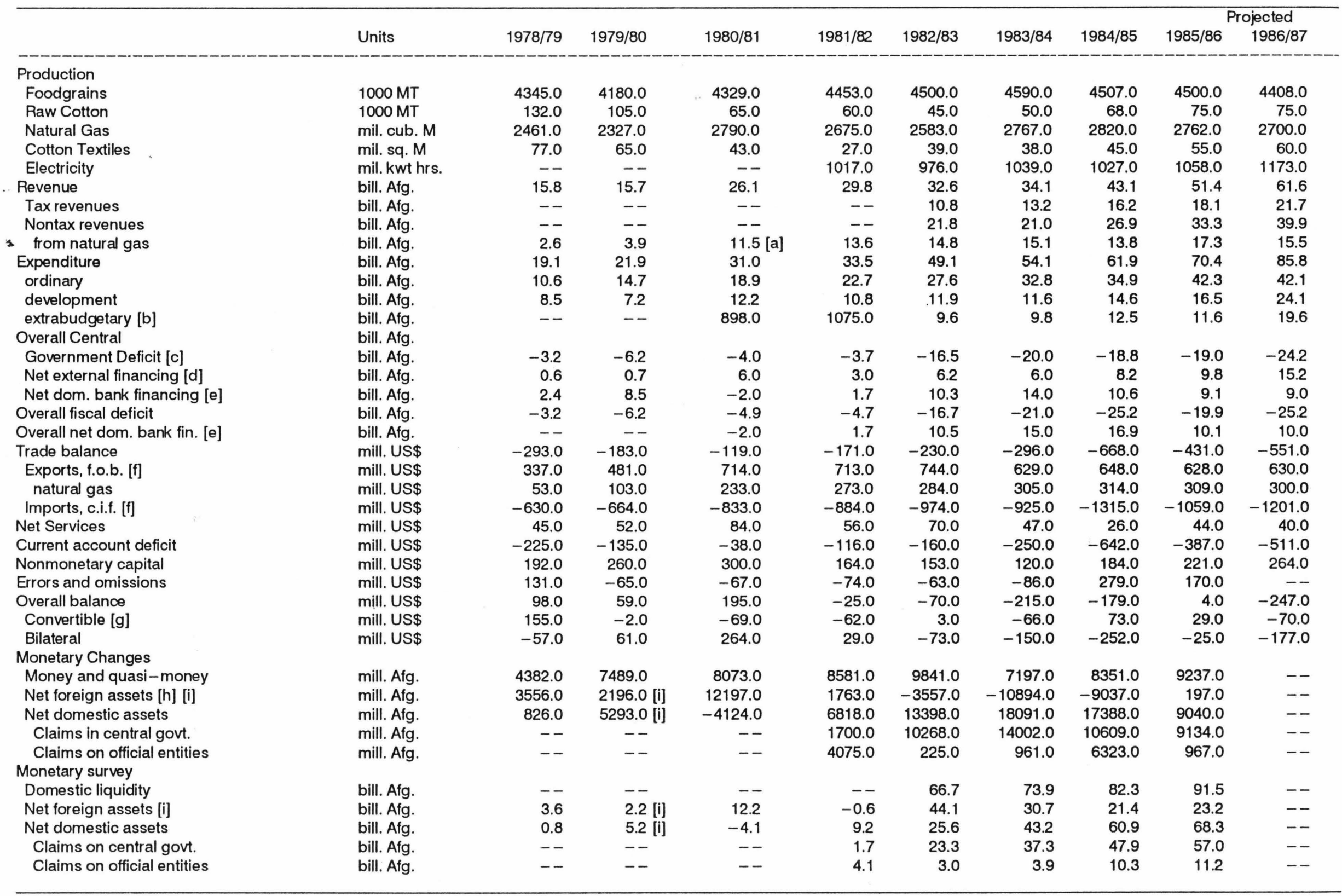


Table A I-3 (continued). Basic IMF Macroeconomic Series from "Recent Economic Developments" Publication

\begin{tabular}{|c|c|c|c|c|c|c|c|c|c|c|}
\hline & Units & $1978 / 79$ & $1979 / 80$ & $1980 / 81$ & $1981 / 82$ & $1982 / 83$ & $1983 / 84$ & $1984 / 85$ & $1985 / 86$ & $\begin{array}{l}\text { Projected } \\
1986 / 87\end{array}$ \\
\hline \multicolumn{11}{|l|}{ Gross official reserves [k] } \\
\hline Convertible assets & mill. \$US & 420.0 & 409.0 & 341.0 & 281.0 & 238.0 & 206.0 & 243.0 & 308.0 & $296.0[1]$ \\
\hline Bilateral assets & mill. \$US & -- & 17.0 & 281.0 & 310.0 & 237.0 & 187.0 & - - & -- & $1.0[$ [I] \\
\hline \multicolumn{11}{|l|}{ External public debt } \\
\hline Amount disbursed & mill. \$US & 1367.0 & 1627.0 & 1927.0 & 2091.0 & 2411.0 & -- & 2576.0 & 2743.0 & -- \\
\hline $\begin{array}{l}\text { Debt service in percent of } \\
\text { goods and services }\end{array}$ & & 144 & & 6.5 & & 150 & 167 & 178 & 100 & 120 \\
\hline \multicolumn{11}{|l|}{ Exchange rate } \\
\hline Average free market rate & Afg./\$US & 38.8 & 42.9 & 46.1 & 56.6 & 76.8 & 99.3 & 122.0 & 136.3 & $144.7[\mathrm{~m}]$ \\
\hline Average official rate & Afg./\$US & -- & -- & -- & 50.2 & 50.6 & 50.6 & 50.6 & 50.6 & $50.6[\mathrm{~m}]$ \\
\hline \multicolumn{11}{|c|}{ Changes in key economic indicators } \\
\hline Net material prod. con. prices & perœent & -- & -- & -- & 1.2 & 1.7 & 4.0 & 1.7 & 0.5 & $.5-1.5$ \\
\hline Kabul price index & perœnt & 5.2 & 9.9 & 12.6 & 21.5 & 16.6 & 19.6 & 27.4 & 9.7 & $5-10$ \\
\hline Net domestic assets & percent & 7.3 & -43.8 & -60.7 & 255.1 & 110.4 & 70.7 & 40.2 & 14.8 & -- \\
\hline Money & percent & -- & -- & -- & 19.1 & 15.9 & 9.4 & 13.8 & 11.8 & -- \\
\hline Money and quasi-money & perœnt & 15.7 & 23.2 & 20.3 & 17.9 & 17.3 & 10.8 & 11.3 & 11.2 & -- \\
\hline
\end{tabular}

[a] Not fully explained by the price and volume increases for gas exports during 1980/81.

[b] Also includes discrepancies due to timing for data from 1982/83-1986/87.

[c] Overall deficits differ from total financing due to data discrepancies and adjustment to cash basis for 1978/79-1981/82 data.

d] Includes external grants for data from 1982/83-1986/87.

[e] As derived from banking statistics for data from 1982/83-1986/87.

[f] See footnote [g] in Table A I-1.

[g] Includes all transactions other than those channeled through bilateral payments accounts for 1978/79-1981/82 data.

[h] The change in net foreign assets here is equivalent to the overall balance in the balance of payments. Any discrepancies between the

change in net foreign assets here and the change in the stock of net foreign assets in the monetary survey are accounted for by valuation and other factors.

[i] Differs from the data on net foreign reserve movements mainly because of the use in the banking statistics of a fixed exchange rate

for the national currency valuation of gold holdings for years 1978/79-1981/82.

[j] Projection.

[k] Excluding gold for data 1982/83-1986/87

[I] September 1986.

[m] Average for the first half of year.

Source: Afghanistan: Recent Economic Developments, IMF, 1983-1986 
PercentagTable 3. Percentage Distribution of Agricultural Land by

Form of Tenenure

\begin{tabular}{|c|c|c|c|c|}
\hline Province & Sharecropped & Mortgaged & Owner operated & Other \\
\hline Kabul & 16.1 & 6.6 & 74.9 & 2.4 \\
\hline Qandahar & 9.1 & 7.5 & 54.1 & 29.3 \\
\hline Herat & 13.4 & 0.7 & 62.7 & 23.2 \\
\hline Mazar-i-Sharif & 23.6 & 1.5 & 60.0 & 14.8 \\
\hline Ningarhar & 5.4 & 43.6 & 45.1 & 5.9 \\
\hline Paktya & 2.0 & 3.7 & 94.3 & - \\
\hline Qataghan & 22.2 & 5.8 & 63.2 & 8.7 \\
\hline Ghazni & 8.1 & 11.2 & 79.6 & 1.1 \\
\hline Girishk & 6.4 & 5.7 & 18.7 & 69.2 \\
\hline Farah & 24.1 & 11.2 & 60.1 & 14.3 \\
\hline Maimana & 2.4 & 0.6 & 75.2 & 21.8 \\
\hline Shibarghan & - & - & - & - \\
\hline Badakhshan & 1.3 & 0.8 & 75.4 & 22.4 \\
\hline Parwan & 5.8 & 9.1 & 80.0 & 5.1 \\
\hline Bamiyan & 1.6 & 0.6 & 73.9 & 23.8 \\
\hline Uruzgan & 13.3 & 5.8 & 79.8 & 1.1 \\
\hline Ghor & 12.1 & 1.2 & 75.8 & 10.9 \\
\hline Average & 13.8 & 5.5 & 60.5 & 20.2 \\
\hline
\end{tabular}

Note: Averages are for all provinces except Shibarghan. Old province names are used.

Source: Ministry of Planning, Survey of Population and Agriculture. Characteristics of 413 Villages in Afghanistan, Table VI, June 1964. 


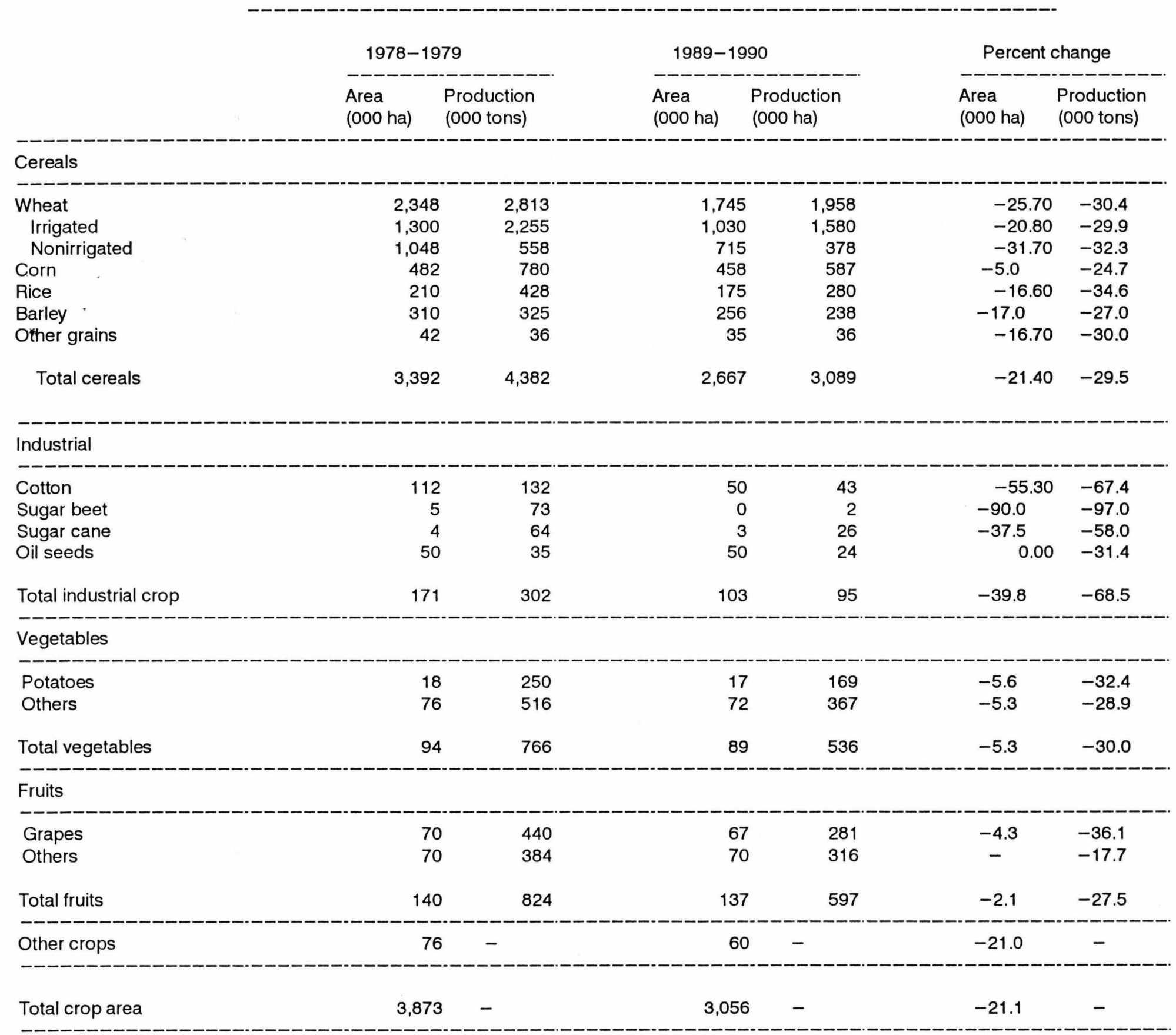

Sources: Statistical Yearbook, Central Statistics Organization, 1989, page 106. Ferogh, A.

in The Current Political and Econnmin. Situation in Afrhanistan 10an, Apnondix A, Tahle A-2 
Shift in Use of Land Resources. 1978-1979 to 1989-1990 (thousand ha)

\begin{tabular}{|c|c|c|c|c|}
\hline \multirow[b]{2}{*}{ Land Classification } & \multicolumn{2}{|c|}{$1978-1979$} & \multicolumn{2}{|c|}{$1989-1990$} \\
\hline & Area & Perœent & Area & Percemt \\
\hline Arable land & 7,900 & 12.0 & 7,910 & 12.0 \\
\hline \multirow{2}{*}{\multicolumn{5}{|c|}{$\begin{array}{l}\text { Cultivated* } \\
\text { Irriqated }\end{array}$}} \\
\hline & & & & \\
\hline Wheat & 1,300 & 2.0 & 1,038 & 1.6 \\
\hline Other cereals & 834 & 1.3 & 742 & 1.1 \\
\hline Fruits and nuts & 141 & 0.2 & 137 & 0.2 \\
\hline Induštrial crops & 134 & 0.2 & 149 & 0.1 \\
\hline${ }^{3}$ Others & 170 & 0.3 & 149 & 0.3 \\
\hline Total irrigated & 2,579 & - & 2,125 & - \\
\hline \multicolumn{5}{|l|}{ Rainfed } \\
\hline Wheat & 1,048 & 1.6 & 715 & 1.1 \\
\hline Other & 246 & 0.4 & 216 & 0.3 \\
\hline Total rainfed & 1,294 & - & 931 & - \\
\hline Total cultivated & 3,873 & 6.0 & 3,056 & 4.7 \\
\hline Uncultivated & 4,037 & 6.0 & 4,854 & 7.3 \\
\hline Total uncultivated & 4,037 & 6.0 & 4,854 & 7.3 \\
\hline Forest & 1,900 & 3.0 & 1,700 & 2.7 \\
\hline Pasture & 40,000 & 61.3 & 40,000 & 61.3 \\
\hline Mountains and desert & 15,453 & 23.7 & 15,653 & 24.0 \\
\hline Total land area & 65,263 & 100.0 & 65,263 & 100.0 \\
\hline
\end{tabular}

*Approximately 25 percent of the irrigated land and 40 percent of the nonirrigated land are fallow in any given year.

Sources: "Afghanistan: Opportunities for Agricultural and Rural

Development, Sector Report", World Bank, 1975, page 8. "Afghanistan:

Journey to Economic Development", Vol.II, World Bank, 1978, Table 7.1

and page 141. Statistical Yearbook, Central Statistics Organization, 
Enterprises with respect to initial and current investments, number

of labours and capital labour ration for public mixed and private sectors

in the year (1977).

\begin{tabular}{|c|c|c|c|c|c|c|c|c|}
\hline \multirow[t]{2}{*}{ Sectors } & \multicolumn{2}{|c|}{ Enterprises } & \multicolumn{2}{|c|}{$\begin{array}{l}\text { Initial investment } \\
\text { (1000 Afs.) }\end{array}$} & \multicolumn{2}{|c|}{$\begin{array}{l}\text { Current investment } \\
\text { (1000 Afs.) }\end{array}$} & Labour & \multirow[t]{2}{*}{ Capita labour ratio } \\
\hline & Numk & ber $\%$ & Number & $\%$ & Number & $\%$ & Number & \\
\hline $\begin{array}{l}\text { Püblic } \\
=\end{array}$ & 35 & 16.13 & 3835134 & 74.0 & 5763716 & 63.04 & 1428739.01 & 403.42 \\
\hline Mixed & 7 & 3.23 & 370912 & 7.2 & 1845392 & 20.18 & $13134 \quad 34.94$ & 240.78 \\
\hline Private & 175 & 80.65 & 970559 & 18.8 & 1533398 & 16.77 & $10170 \quad 27.05$ & 150.78 \\
\hline Total & 217 & 100 & 5177405 & 100 & 9142506 & 100 & 37591100 & 243.21 \\
\hline
\end{tabular}

Source: Statistical information of Afghanistan (1975 - 1977) (45) 
1. Comparative Nat'I Prod. Est.

2. UNDP Adj. GDP

3. IBRD Adjusted GNP Est.

4. IBRD GDP and GNP Adj.

5. IBRD Est. of Exp. on GDP

6. National Income (GOA)

7. GNP at Market Prices

8. RRNA Adjustment of GDP

9. RRNA Adj. Crop Production

10. USDA Series on Ag. Prod.

11. GOA Est. of Crop Prod.

12. Trends in Crop Production

13. Pre-war Area of Crop Prod.

14. Pre-war Ag. Production

15. Wheat Production (East)
16. Area of Cultivation (East)

17. Livestock Pop. (East)

18. Wheat Production (North)

19. Cotton Production (North)

20. Area of Cultivation (North)

21. Livestock Pop. (North)

22. Pre-war Ag. Co-ops

23. Pct. of Cult./Irrigated Land

24. UN Proj. of Essen. Commodity Req.

25. Ind. and Mining Prod.(UN Series)

26. Ind. and Mining Prod.(IMF Series)

27. Pre-war Coal Production

28. Natural Gas Production

O. RETURN TO MAIN MENU 
Table A II-1. Comparative National Product Estimates

(billions of atghanis) [a]

\begin{tabular}{|c|c|c|c|c|c|c|c|c|c|c|c|c|c|c|c|c|c|c|c|}
\hline & $1968 / 691$ & 59/70 & $1970 / 71$ & $1971 / 72$ & 1972/73 & $1973 / 74$ & $1974 / 75$ & $1975 / 76$ & $1976 / 77$ & $1977 / 78$ & $1978 / 79$ & $1979 / 80$ & $1980 / 81$ & $1981 / 82$ & $1982 / 83$ & $1983 / 84$ & $1984 / 85$ & $1985 / 86$ & $1986 / 87$ \\
\hline Official GOA NMP & 69.99 & -- & -- & -- & 63.75 & -- & -- & 80.95 & -- & -1 & -- & -- & -- & 93.20 & 94.80 & 98.60 & 100.20 & 99.90 & 104.00 \\
\hline UNDP Adj. GDP & -- & -- & -- & -- & -- & -- & -1 & -- & -- & -1 & 117.20 & -- & -- & -- & -- & -- & 107.50 & 105.60 & 107.70 \\
\hline RRNA Adj. UNDP GDP & -- & -- & -- & -- & -- & -- & -1 & -- & -- & -1 & 113.70 & 99.12 & 91.63 & 91.52 & 89.52 & 85.80 & 86.16 & 82.42 & 80.97 \\
\hline World Bank GDP & 89.01 & 90.37 & 92.23 & 87.82 & 86.17 & 95.98 & 103.41 & 106.50 & 113.22 & - & -- & -- & -- & -- & -- & -- & -- & -- & -- \\
\hline World Bank GNP & 88.61 & 89.99 & 91.82 & 87.38 & 85.60 & 95.53 & 103.98 & 108.77 & 115.33 & -1 & -- & -- & -- & -- & -- & -- & -- & -- & -- \\
\hline
\end{tabular}

[a] Data through $75 / 76$ are in constant $75 / 76$ prices; all other data are in constant 78/79 prices.

3 Source: UNDP Discussion Paper on Afghanistan of April 1988; Appendix Tables A I-1, A \|-2, A \|-3, A I-5, A I-6. 
Table A II-2. UNDP Estimates of Adjusted Gross Domestic Product at Constant 1978/79 Prices (millions of afghanis, unless otherwise indicated)

\begin{tabular}{|c|c|c|c|c|c|c|}
\hline & $1978-79$ & $1984-85$ & $1985-86$ & $1986-87$ & $\begin{array}{l}\text { Share } \\
\text { of GDP } \\
1986-87\end{array}$ & $\begin{array}{l}\text { Avg. annual } \\
\text { growth rate } \\
1978 / 79-1986 / 87\end{array}$ \\
\hline \multicolumn{7}{|l|}{ 1. Agriculture, Livestock } \\
\hline \& Forestry & 61,900 & 50,758 & 49,520 & 49,000 & 45.5 & -2.6 \\
\hline Crops & 42,158 & 34,569 & 33,726 & 33,350 & 31.0 & -2.8 \\
\hline Livestock & 19,441 & 15,942 & 15,553 & 15,415 & 14.2 & -2.8 \\
\hline Forestry & 301 & 247 & 241 & 235 & 0.3 & -2.8 \\
\hline \multicolumn{7}{|l|}{ 2. Mining Ind ustry } \\
\hline \& Energy & 14,400 & 13,428 & 14,321 & 14,651 & 13.6 & 0.2 \\
\hline Mining & 1,550 & 1,761 & 1,688 & 1,525 & 1.4 & -0.2 \\
\hline Industry & 3,909 & 4,029 & 5,137 & 5,699 & 5.3 & 4.9 \\
\hline Electricity & 376 & 438 & 473 & 527 & 0.5 & 4.3 \\
\hline Handicrafts & 8,565 & 7,200 & 7,023 & 6,900 & 6.4 & -2.4 \\
\hline 3. Construction & 4,300 & 3,900 & 4,000 & 4,800 & 4.4 & 1.4 \\
\hline \multicolumn{7}{|l|}{ 4. Transport } \\
\hline \& Communications & 3,500 & 3,700 & 2,700 & 2,900 & 2.7 & -2.2 \\
\hline 5. Trade \& Distribution & 9,700 & 9,100 & 9,000 & 9,100 & 8.5 & -1.0 \\
\hline 6. Others & 1,700 & 1,800 & 1,700 & 1,700 & 1.6 & \\
\hline 7. Total Production & 95,500 & 82,686 & 81,241 & 82,151 & 76.4 & -2.0 \\
\hline 8. Services & 9,275 & 12,575 & 12,138 & 13,242 & 12.3 & 4.5 \\
\hline Private $(5 \%$ of 7$)$ & 4,775 & 4,150 & 4,050 & 4,102 & 3.8 & -2.0 \\
\hline Public & 4,500 & 8,425 & 8,088 & 9,140 & 8.5 & 9.3 \\
\hline 9. Housing $(6 \%$ of 7$)$ & 5,730 & 4,980 & 4,860 & 4,922 & 4.6 & -2.1 \\
\hline \multicolumn{7}{|l|}{ 10. Total Net Domestic } \\
\hline Product at factor cost & 110,505 & 100,241 & 98,239 & 100,315 & -- & -- \\
\hline 11. Depreciation & 6,700 & 7,300 & 7,400 & 7,400 & 6.8 & -- \\
\hline \multicolumn{7}{|l|}{ 12. Total Gross Domestic } \\
\hline Product at factor cost & 117,205 & 107,541 & 105,639 & 107,715 & 100.0 & -1.0 \\
\hline
\end{tabular}

UNDP Note 1. With the exception of GDP of Construction and Transport and Communications, the figures presented in this table differ from official data as they have been adjusted to reflect the

depressed situation of the economy over the period. Official figures are given in Annex Table 1 of

the UNDP Discussion Paper on Afghanistan of April 1988.

UNDP Note 2. The Government methodology does not provide figures on the GDP of the services sector.

In order to give a complete picture of GDP at factor cost above these flows were added using the

methodology presented in the table. The Government GDP, mainly wages and salaries, has been expressed

at constant prices using the consumer price index.

Sources: Original data from Central Statistical Office; cited from the UNDP Discussion Paper on 
Table A II-3. World Bank Adjusted GNP Estimates by Sector (constant $1975 / 76$ prices)

(billions of afghanis)

\begin{tabular}{|c|c|c|c|c|c|c|c|c|c|c|}
\hline & $1968 / 69$ & $1969 / 70$ & $1970 / 71$ & $1971 / 72$ & $1972 / 73$ & $1973 / 74$ & $1974 / 75$ & $1975 / 76$ & $1976 / 77$ & $1982 / 83$ * \\
\hline Agriculture \& livestock & 51.03 & & & & 43.38 & & & 53.81 & & 73.86 \\
\hline Crop & 31.56 & & & & 31.20 & & & 36.08 & & 47.65 \\
\hline Livestock & 18.81 & & & & 11.48 & & & 17.03 & & 25.45 \\
\hline Forestry & 0.66 & & & & 0.70 & & & 0.70 & & 0.76 \\
\hline Industry \& mining & 8.01 & & & & 9.70 & & & 11.10 & & 17.97 \\
\hline Mining & 0.33 & & & & 0.50 & & & 0.73 & & 1.33 \\
\hline Electricity & 0.10 & & & & 0.14 & & & 0.19 & & 0.62 \\
\hline Manufacturing & 1.12 & & & & 1.73 & & & 2.48 & & 6.32 \\
\hline Handicrafts & 6.46 & & & & 7.33 & & & 7.70 & & 9.70 \\
\hline Construction & 1.43 & & & & 1.39 & & & 2.17 & & 6.10 \\
\hline Transport \& comm. & 2.31 & & & & 3.12 & & & 3.46 & & 5.51 \\
\hline Transport & 2.23 & & & & 2.97 & & & 3.29 & & 5.09 \\
\hline Communications & 0.08 & & & & 0.15 & & & 0.17 & & 0.42 \\
\hline Trade \& food dist. & 8.19 & & & & 9.18 & & & 11.82 & & 19.66 \\
\hline Others & 0.98 & & & & 1.05 & & & 1.09 & & 1.29 \\
\hline Total production & 71.95 & & & & 67.82 & & & 83.45 & & 124.39 \\
\hline Services & 5.98 & & & & 6.26 & & & 8.64 & & 14.69 \\
\hline Private & 3.32 & & & & 3.07 & & & 3.81 & & 5.76 \\
\hline Public & 2.66 & & & & 3.19 & & & 4.83 & & 8.93 \\
\hline Housing & 4.33 & & & & 4.15 & & & 5.00 & & 7.70 \\
\hline NDP at factor cost & 82.26 & 83.00 & 84.50 & 80.00 & 78.23 & 87.50 & 92.60 & 97.09 & 104.74 & 146.78 \\
\hline Depreciation & 4.20 & 4.35 & 4.50 & 4.66 & 4.80 & 4.94 & 5.10 & 5.30 & 5.6 & 9.42 \\
\hline GDP at factor cost & 86.46 & 87.35 & 89.00 & 84.66 & 83.03 & 92.44 & 97.70 & 102.39 & 110.34 & 156.20 \\
\hline Indirect taxes & 2.92 & 3.47 & 3.73 & 3.81 & 3.84 & 4.34 & 6.76 & 6.01 & 6.51 & 10.84 \\
\hline Subsidy & -0.37 & -0.45 & -0.50 & -0.65 & -0.70 & -0.80 & -1.05 & -1.90 & -3.63 & -4.45 \\
\hline GDP at market prices & 89.01 & 90.37 & 92.23 & 87.82 & 86.17 & 95.98 & 103.41 & 106.50 & 113.22 & 162.59 \\
\hline Factor income & -0.40 & -0.38 & -0.41 & -0.44 & -0.42 & -0.45 & 0.51 & 2.27 & 2.11 & -- \\
\hline GNP at market prices & 88.61 & 89.99 & 91.82 & 87.38 & 85.75 & 95.53 & 103.92 & 108.77 & 115.33 & -- \\
\hline
\end{tabular}

* World Bank projections.

Note: Refer to Table A II-4 for derivation of adjustments.

Source: Ministry of Planning and World Bank Mission estimates; cited

from "Afahanistan. The Journev to Economic Develonment" Vol II March 
Table A II-4. World Bank Adjy stments n Derivation of GDP and GNP Estimates
(billions of Atghanis at 1975/76 con stant prices)

Table A All-4 (continued). World Bank Adjustments $h$ Derivation of GDP and GNP Estmates
(billions of Afghanis at 1075/78 constant prices)

\begin{tabular}{|c|c|c|c|c|c|c|c|c|c|c|c|c|c|c|c|c|c|c|}
\hline & \multicolumn{7}{|c|}{$\begin{array}{l}\text { (billions of Atghanis at 1975/78 con stant prices) } \\
\text { 1068/50 }\end{array}$} & & \multicolumn{10}{|c|}{ (billions of Atghanis at 1075/78 constant prices) } \\
\hline & $\begin{array}{l}\text { Total } \\
\text { Prod. }\end{array}$ & $\begin{array}{l}\text { Adj. } \\
\text { Factor }\end{array}$ & $\begin{array}{l}\text { Adj. Tot. } \\
\text { Prod. }\end{array}$ & $\begin{array}{l}\text { Materiat } \\
\text { Exp. }\end{array}$ & $\begin{array}{l}1968860 \\
\text { Adj. } \\
\text { Factor }\end{array}$ & $\begin{array}{l}\text { Adj. Mat. } \\
\text { Exp. }\end{array}$ & $\begin{array}{l}\text { Net } \\
\text { Prod. }\end{array}$ & $\begin{array}{l}\text { Adj. } \\
\text { Factor }\end{array}$ & $\begin{array}{l}\text { Adj. Not } \\
\text { Prod. }\end{array}$ & $\begin{array}{l}\text { Total } \\
\text { Prod. }\end{array}$ & $\begin{array}{l}\text { Adj. } \\
\text { Factor }\end{array}$ & $\begin{array}{l}\text { Adj. Tot } \\
\text { Prod. }\end{array}$ & $\begin{array}{c}\text { Matarilad } \\
\text { Exp. }\end{array}$ & $\begin{array}{c}197273 \\
\text { Adl. } \\
\text { Factor }\end{array}$ & $\begin{array}{l}\text { Ad]. Mat. } \\
\text { Exp. }\end{array}$ & $\begin{array}{l}\text { Not } \\
\text { Prod. }\end{array}$ & $\begin{array}{l}\text { Adj. } \\
\text { Factor }\end{array}$ & $\begin{array}{l}\text { Adj. Not } \\
\text { Prod. }\end{array}$ \\
\hline Crops & 43.95 & 1.00 & 43.95 & 12.39 & 1.00 & 12.30 & 31.56 & 1.00 & 31.56 & 43.85 & 1.00 & 43.85 & 12.05 & 1.00 & 12.05 & 31.20 & 1.00 & 31.20 \\
\hline Uvestock & 22.13 & 1.00 & 22.13 & 3.32 & 1.00 & 3.32 & 18.81 & 1.00 & 18.81 & 13.15 & 1.00 & 13.15 & 1.67 & 1.00 & 1.07 & 11.48 & 1.00 & 11.48 \\
\hline $\begin{array}{l}\text { Forestry } \\
\text { Total }\end{array}$ & $\begin{array}{r}0.609 \\
86.77\end{array}$ & 1.00 & $\begin{array}{r}0.60 \\
68.77\end{array}$ & $\begin{array}{r}0.03 \\
15.74\end{array}$ & 1.00 & $\begin{array}{r}0.03 \\
15.74\end{array}$ & $\begin{array}{r}0.00 \\
51.03\end{array}$ & 1.00 & $\begin{array}{r}0.66 \\
51.03\end{array}$ & $\begin{array}{r}0.73 \\
57.73\end{array}$ & 1.00 & $\begin{array}{r}0.73 \\
57.73\end{array}$ & $\begin{array}{r}0.03 \\
14.35\end{array}$ & 1.00 & $\begin{array}{r}0.03 \\
14.35\end{array}$ & $\begin{array}{r}0.70 \\
43.38\end{array}$ & 1.00 & $\begin{array}{r}0.70 \\
43.38\end{array}$ \\
\hline ndusty & & & & & & & & & & & & & & & & & & \\
\hline Public and mixed sector & 3.62 & 1.00 & 3.62 & 2.25 & 1.00 & 2.25 & 1.37 & 1.00 & 1.37 & 5.81 & 1.00 & 5.81 & 3.75 & 1.00 & 3.75 & 2.00 & 1.00 & 2.00 \\
\hline Private & 0.99 & 1.00 & 0.99 & 0.81 & 1.00 & 0.81 & 0.18 & 1.00 & 0.18 & 1.65 & 1.00 & 1.65 & 1.34 & 1.00 & 1.34 & 0.31 & 1.00 & 0.31 \\
\hline $\begin{array}{l}\text { Handicratts } \\
\text { Total }\end{array}$ & $\begin{array}{l}10.77 \\
15.38\end{array}$ & 1.00 & $\begin{array}{l}10.77 \\
15.38\end{array}$ & $\begin{array}{r}8.19 \\
11.25\end{array}$ & 0.40 & $\begin{array}{l}4.31 \\
7.37\end{array}$ & $\begin{array}{l}2.58 \\
4.13\end{array}$ & 2.50 & $\begin{array}{l}6.46 \\
8.01\end{array}$ & $\begin{array}{l}12.21 \\
19.67\end{array}$ & 1.00 & $\begin{array}{l}12.21 \\
19.67\end{array}$ & $\begin{array}{r}9.28 \\
14.37\end{array}$ & 0.40 & $\begin{array}{l}4.88 \\
0.97\end{array}$ & $\begin{array}{l}2.93 \\
5.30\end{array}$ & 2.80 & $\begin{array}{l}7.33 \\
0.70\end{array}$ \\
\hline Construction & & & & & & & & & & & & & & & & & & \\
\hline Dev. Plan - Public & $\begin{array}{l}2.15 \\
0.03\end{array}$ & 1.00 & & & & & & & & 2.39 & 1.00 & 2.39 & & & & & & \\
\hline Oher private Private & $\begin{array}{l}0.03 \\
1.18\end{array}$ & $\begin{array}{l}1.00 \\
1.00\end{array}$ & $\begin{array}{l}0.03 \\
1.18\end{array}$ & $\begin{array}{l}1.33 \\
0.60\end{array}$ & $\begin{array}{l}1.00 \\
1.00\end{array}$ & 1.33 & $\begin{array}{l}0.85 \\
0.58\end{array}$ & 1.00 & $\begin{array}{l}0.85 \\
0.58\end{array}$ & $\begin{array}{l}0.10 \\
0.88\end{array}$ & 1.00 & 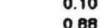 & $\begin{array}{l}1.53 \\
0.5\end{array}$ & 1.00 & $\begin{array}{l}1.53 \\
0.45\end{array}$ & 0.06 & 1.00 & 0.06 \\
\hline Total & $\begin{array}{l}1.18 \\
3.36\end{array}$ & & $\begin{array}{l}1.18 \\
3.36\end{array}$ & $\begin{array}{l}0.60 \\
1.03\end{array}$ & & $\begin{array}{l}0.60 \\
1.03\end{array}$ & $\begin{array}{l}0.58 \\
1.43\end{array}$ & & $\begin{array}{l}0.58 \\
1.43\end{array}$ & $\begin{array}{l}0.88 \\
\mathbf{3 . 3 7}\end{array}$ & & $\begin{array}{l}0.88 \\
3.37\end{array}$ & $\begin{array}{l}0.45 \\
1.08\end{array}$ & 1.00 & $\begin{array}{l}0.45 \\
1.08\end{array}$ & $\begin{array}{l}0.43 \\
1.30\end{array}$ & & $\begin{array}{l}0.43 \\
1.39\end{array}$ \\
\hline Transport & & & & & & & & & & & & & & & & & & \\
\hline Road & 1.58 & 2.00 & $\begin{array}{l}3.16 \\
075\end{array}$ & 0.83 & 2.00 & 1.60 & 0.75 & 2.00 & 1.50 & 2.08 & 2.00 & 4.16 & 1.09 & 2.00 & 2.18 & 0.99 & 2.00 & \\
\hline Air & 0.75 & 1.00 & 0.75 & 0.04 & 1.00 & 0.04 & 0.71 & 1.00 & 0.71 & 1.00 & 1.00 & 1.00 & 0.04 & 1.00 & 0.04 & 0.96 & 1.00 & 0.96 \\
\hline $\begin{array}{c}\text { Water } \\
\text { Total }\end{array}$ & $\begin{array}{l}0.04 \\
2.37\end{array}$ & 1.00 & $\begin{array}{l}0.04 \\
3.95\end{array}$ & $\begin{array}{l}0.01 \\
0.88\end{array}$ & 1.00 & 0.01 & $\begin{array}{l}0.03 \\
1.40\end{array}$ & 1.00 & 0.03 & 0.04 & 1.00 & 0.04 & 0.01 & 1.00 & 0.01 & 0.03 & 1.00 & 0.03 \\
\hline Total & 2.37 & & 3.95 & 0.88 & & 1.71 & 1.40 & & 2.24 & 3.12 & & 5.20 & 1.14 & & 2.23 & 1.98 & & 2.97 \\
\hline - Communications & & & & & & & & & & & & & & & & & & \\
\hline Telephone & 0.05 & 1.00 & 0.05 & 0.01 & 1.00 & 0.01 & 0.04 & 1.00 & 0.04 & 0.09 & 1.00 & 0.09 & 0.02 & 1.00 & 0.02 & 0.07 & 1.00 & 0.07 \\
\hline $\begin{array}{l}\text { Postal service } \\
\text { Oyersens }\end{array}$ & 0.02 & 1.00 & 0.02 & 0.00 & 1.00 & 0.00 & 0.01 & 1.00 & 0.01 & 0.03 & 1.00 & 0.03 & 0.01 & 1.00 & 0.01 & 0.03 & 1.00 & 0.03 \\
\hline $\begin{array}{l}\text { Overssas phone } \\
\text { Total }\end{array}$ & $\begin{array}{l}0.04 \\
0.10\end{array}$ & $\begin{array}{l}1.00 \\
1.00\end{array}$ & $\begin{array}{l}0.04 \\
0.10\end{array}$ & $\begin{array}{l}0.01 \\
0.03\end{array}$ & 1.00 & $\begin{array}{l}0.01 \\
0.03-3-3-1\end{array}$ & $\begin{array}{l}0.03 \\
0.08\end{array}$ & $\begin{array}{l}1.00 \\
.00\end{array}$ & $\begin{array}{l}0.03 \\
0.08\end{array}-3-3$ & $\begin{array}{l}0.07 \\
0.20\end{array}$ & 1.00 & $\begin{array}{l}0.07 \\
0.20\end{array}$ & $\begin{array}{l}0.02 \\
0.05\end{array}-3-3$ & 1.00 & $\begin{array}{l}0.02 \\
0.05\end{array}$ & $\begin{array}{l}0.05 \\
0.05\end{array}$ & 1.00 & ${ }_{0.05}^{0.05}>>-15$ \\
\hline $\begin{array}{l}\text { lotal (excl. community service) } \\
\text { Total (ext) }\end{array}$ & $\begin{array}{l}0.10 \\
0.04\end{array}$ & $\begin{array}{l}1.00 \\
1.00\end{array}$ & $\begin{array}{l}0.10 \\
0.04\end{array}$ & $\begin{array}{l}0.03 \\
0.01\end{array}$ & $\begin{array}{l}1.00 \\
1.00\end{array}-10$ & $\begin{array}{l}0.03 \\
0.01\end{array}$ & $\begin{array}{l}0.08 \\
0.03\end{array}$ & $\begin{array}{l}1.00 \\
1.00\end{array}$ & $\begin{array}{l}0.08 \\
0.03\end{array}-3$ & $\begin{array}{l}0.07 \\
0.07\end{array}$ & $\begin{array}{l}1.00 \\
1.00\end{array}-10$ & $\begin{array}{l}0.20 \\
0.07\end{array}$ & $\begin{array}{l}0.05 \\
0.02\end{array}$ & $\begin{array}{l}1.00 \\
1.00\end{array}$ & $\begin{array}{l}0.05 \\
0.02\end{array}$ & $\begin{array}{l}0.15 \\
0.05\end{array}$ & 1.00 & 0.15 \\
\hline Community sorvices & 0.00 & 1.00 & 0.00 & 0.02 & 1.00 & 0.02 & 0.05 & 1.00 & 0.05 & 0.13 & 1.00 & 0.13 & 0.03 & 1.00 & 0.03 & 0.10 & 1.00 & 0.10 \\
\hline Trade, food distibution & & & & & & & & & & & & & & & & & & \\
\hline Food trade & 4.41 & 1.00 & 4.41 & 1.07 & 1.00 & 1.07 & 3.34 & 1.00 & 3.34 & 4.23 & 1.00 & 4.23 & 1.04 & 1.00 & 1.04 & 3.19 & 1.00 & 3.19 \\
\hline Gov't food distribution & 0.16 & 1.00 & 0.16 & 0.04 & 1.00 & 0.04 & 0.12 & 1.00 & 0.12 & 0.18 & 1.00 & 0.18 & 0.04 & 1.00 & 0.04 & 0.14 & 1.00 & 0.14 \\
\hline $\begin{array}{l}\text { ndustial products } \\
\text { Handitrafts }\end{array}$ & $\begin{array}{l}0.02 \\
1.08\end{array}$ & $\begin{array}{l}1.00 \\
2000\end{array}$ & $\begin{array}{l}0.92 \\
2.16\end{array}$ & $\begin{array}{l}0.22 \\
0.26\end{array}$ & $\begin{array}{l}1.00 \\
200\end{array}$ & 0.22 & $\begin{array}{l}0.70 \\
0.82\end{array}$ & $\begin{array}{l}1.00 \\
200\end{array}$ & 0.70 & 1.49 & $\begin{array}{l}1.00 \\
200\end{array}$ & 1.49 & 0.37 & 1.00 & 0.37 & 1.12 & 1.00 & 1.12 \\
\hline $\begin{array}{l}\text { Mandicrafts } \\
\text { Imported goods }\end{array}$ & $\begin{array}{l}1.08 \\
1.21\end{array}$ & $\begin{array}{l}2.00 \\
1.00\end{array}$ & $\begin{array}{l}2.16 \\
1.21\end{array}$ & $\begin{array}{l}0.26 \\
0.29\end{array}$ & $\begin{array}{l}2.00 \\
1.00\end{array}$ & $\begin{array}{l}0.52 \\
0.29\end{array}-\mathrm{l} \mathrm{l}$ & $\begin{array}{l}0.82 \\
0.92\end{array}$ & $\begin{array}{l}2.00 \\
1.00\end{array}$ & $\begin{array}{l}1.04 \\
0.92\end{array}$ & $\begin{array}{l}1.22 \\
1.64\end{array}$ & $\begin{array}{l}2.00 \\
1.00\end{array}$ & $\begin{array}{l}2.44 \\
1.64\end{array}$ & $\begin{array}{l}0.30 \\
0.40\end{array}-30$ & 2.00 & $\begin{array}{l}0.00 \\
0.40\end{array}$ & 1.24 & 2.00 & $\begin{array}{l}1.84 \\
1.24\end{array}$ \\
\hline $\begin{array}{l}\text { Impotrod goods } \\
\text { Total }\end{array}$ & $\begin{array}{l}1.21 \\
7.78\end{array}$ & & 8.80 & $\begin{array}{l}0.29 \\
1.80\end{array}$ & & $\begin{array}{l}0.29 \\
2.15\end{array}-1$ & 5.80 & & 0.71 & 8.70 & & 0.08 & 2.15 & & 2.46 & 0.01 & & $\begin{array}{l}1.24 \\
7.52\end{array}$ \\
\hline Oher goods $(+25 \%)$ & 1.05 & 1.00 & 1.05 & 0.47 & 1.00 & 0.47 & 1.48 & 1.00 & 1.48 & $\begin{array}{r}2.19 \\
-1.09\end{array}$ & 1.00 & $\begin{array}{r}2.19 \\
12.17\end{array}$ & 0.54 & 1.00 & 0.54 & 1.05 & 1.00 & 1.05 \\
\hline & & & & & & & & & & & & & & & & & & \\
\hline Ohers & 1.46 & 1.00 & 1.48 & 0.48 & 1.00 & 0.48 & 0.08 & 1.00 & 0.98 & 1.60 & 1.00 & 1.80 & 0.61 & 1.00 & 0.01 & 1.05 & 1.00 & 1.05 \\
\hline Total production & 99.17 & & 101.83 & 32.67 & & 29.88 & 66.50 & & 71.95 & 96.70 & & 100.00 & 35.20 & & 32.10 & 01.50 & & 07.81 \\
\hline $\begin{array}{l}\text { Services } \\
\text { Piturat }\end{array}$ & & & & & & & 5.88 & & 5.98 & & & & & & & 0.14 & & 0.26 \\
\hline $\begin{array}{l}\text { Private |a| } \\
\text { Public |b| }\end{array}$ & & & & & & & $\begin{array}{l}3.32 \\
2.36\end{array}$ & $\begin{array}{l}1.00 \\
1.04\end{array}$ & $\begin{array}{l}3.32 \\
2.66\end{array}$ & & & & & & & $\begin{array}{l}3.07 \\
3.07\end{array}$ & $\begin{array}{l}1.00 \\
1.04\end{array}$ & $\begin{array}{l}3.07 \\
3.19\end{array}$ \\
\hline Housing $\mathrm{kl}$ & & & & & & & 4.33 & 1.00 & 4.33 & & & & & & & 4.15 & 1.00 & 4.15 \\
\hline NDP at fector cost & & & & & & & 76.71 & & 82.26 & & & & & & & 71.70 & & 78.22 \\
\hline Depreciation $|d|$ & & & & & & & 1.53 & 2.75 & 4.20 & & & & & & & 1.47 & 3.27 & 4.80 \\
\hline GDP at factor cost & & & & & & & 78.24 & & 86.40 & & & & & & & 73.26 & & 83.02 \\
\hline ndirect taxes & & & & & & & 2.02 & 1.00 & 2.92 & & & & & & & 3.84 & 1.00 & 3.84 \\
\hline Subsidy (-) & & & & & & & 0.37 & 1.00 & 0.37 & & & & & & & 0.70 & 1.00 & 0.70 \\
\hline Factor income $(-)|e|$ & & & & & & & 0.06 & -0.62 & -0.40 & & & & & & & 0.10 & -4.20 & -0.42 \\
\hline GNP at market prices & & & & & & & 80.85 & & 88.61 & & & & & & & 76.50 & & 85.74 \\
\hline
\end{tabular}

"World Bank mission projection.

[a] $5 \%$ of total material product

the

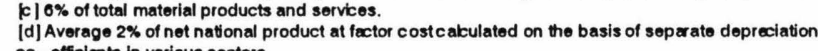

co- efficlents in various sectors.
leI Interst on public debt is used as proxy factor income assuming that the other inflows and outfiows are
balenced.

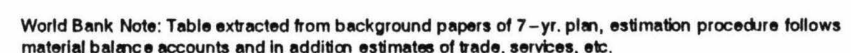

Sources: GOA Ministry of Planning; cited from "Afghanistan: The Journey to Economic Development, Vol.
II, March 1978, World Bank. 
Table A II- -4 (continued). World Bank Adis stments h Derivation of GDP and GNP Estimates
(billions of Atghanis at $1975 / 70$ con stant prices)

Table A Ill-4 (continued). World Bank Adjy stments h Derivation of GDP and GNP Estimates

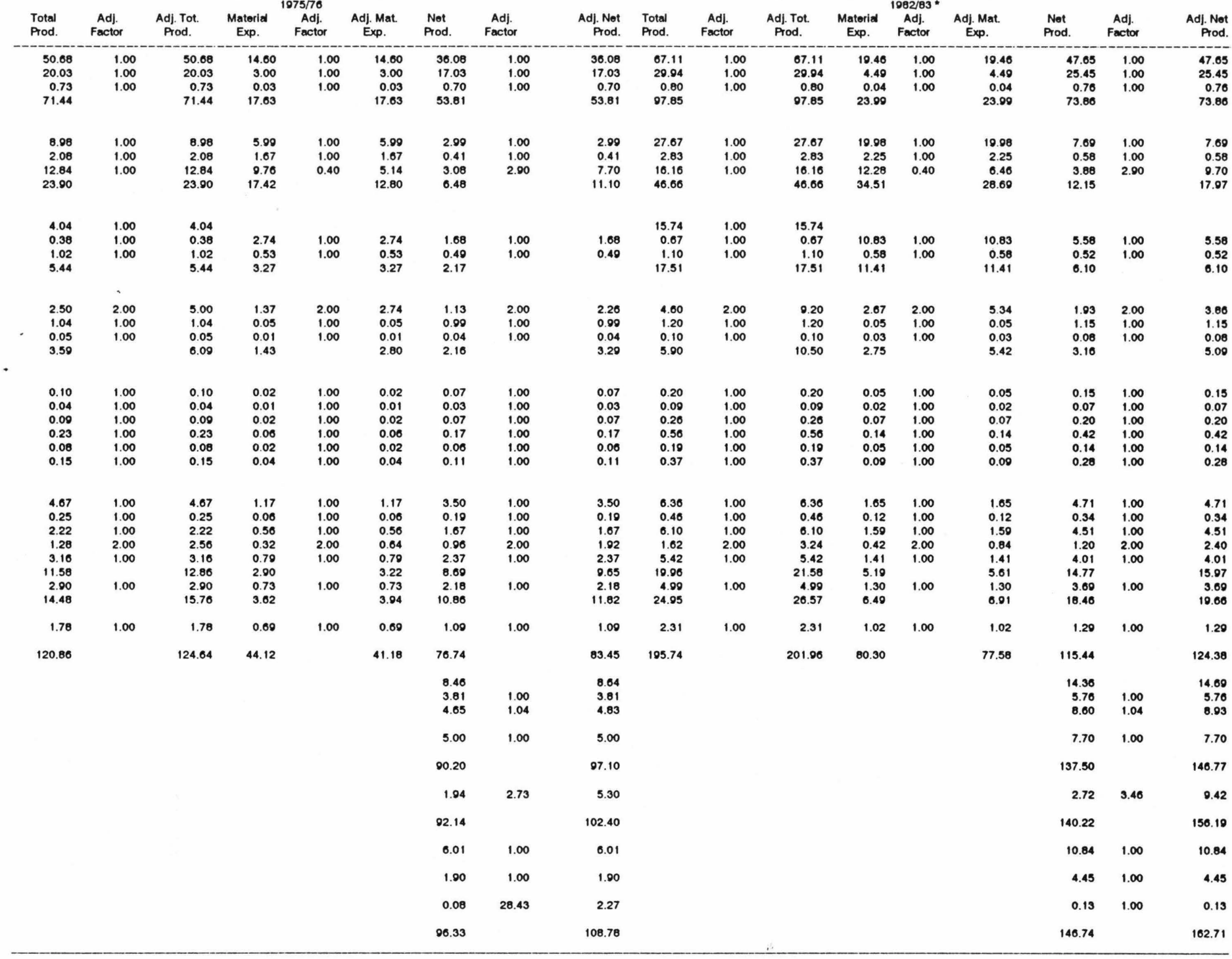




\section{Bollions of a dghaniss}

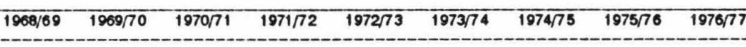

\begin{tabular}{|c|c|c|c|c|c|c|c|c|c|}
\hline Privatio consumption & 81.51 & 81.32 & 82.14 & 78.63 & 76.31 & 84.55 & 90.69 & 91.67 & 92.2 \\
\hline Government consumption & 3.42 & 3.67 & 3.83 & 3.85 & 4.09 & 4.32 & 4.38 & 6.13 & 6.35 \\
\hline $\begin{array}{l}\text { Gross Invostment } \\
\text { Private } \\
\text { Public }\end{array}$ & $\begin{array}{l}2.06 \\
4.00\end{array}$ & $\begin{array}{l}3.00 \\
4.50\end{array}$ & $\begin{array}{l}3.00 \\
4.50\end{array}$ & $\begin{array}{l}3.00 \\
5.00\end{array}$ & $\begin{array}{l}2.73 \\
4.20\end{array}$ & $\begin{array}{l}3.00 \\
4.00\end{array}$ & $\begin{array}{l}3.50 \\
5.50\end{array}$ & $\begin{array}{l}4.03 \\
6.40\end{array}$ & $\begin{array}{r}4.1 \\
11.45\end{array}$ \\
\hline $\begin{array}{l}\text { Export of goods } \\
\text { Less import of goods }\end{array}$ & $\begin{array}{l}4.12 \\
7.03\end{array}$ & $\begin{array}{l}4.49 \\
6.05\end{array}$ & $\begin{array}{l}4.65 \\
6.08\end{array}$ & $\begin{array}{l}5.99 \\
8.90\end{array}$ & $\begin{array}{l}7.10 \\
8.63\end{array}$ & $\begin{array}{l}9.09 \\
0.19\end{array}$ & $\begin{array}{l}12.04 \\
13.46\end{array}$ & $\begin{array}{l}\begin{array}{l}13.42 \\
15.20\end{array} \\
\end{array}$ & $\begin{array}{l}17.67 \\
18.67\end{array}$ \\
\hline $\begin{array}{l}\text { Net meome trom non - factor } \\
\text { senvices }\end{array}$ & 0.04 & 0.04 & 0.19 & 0.25 & 0.28 & 0.21 & 0.16 & 0.05 & 0.12 \\
\hline $\begin{array}{l}\text { Total axpenditure on GDP } \\
\text { at market prices }\end{array}$ & 89.01 & $\$ 0.37$ & 02.23 & 87.82 & 86.17 & 95.98 & 103.41 & 106.50 & 113.22 \\
\hline
\end{tabular}

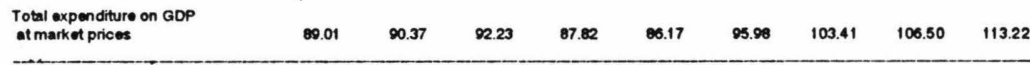

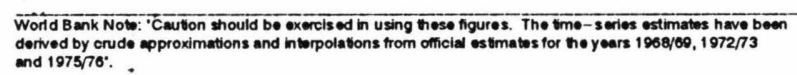

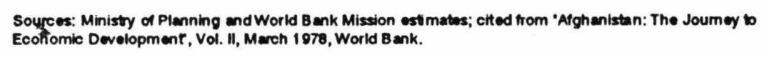

Table All- B. World Benk Estomatess G Expen diture on GDP

\begin{tabular}{|c|c|c|c|c|c|c|c|c|c|c|c|c|c|c|c|c|c|c|c|c|c|c|c|c|c|}
\hline \multicolumn{26}{|c|}{ 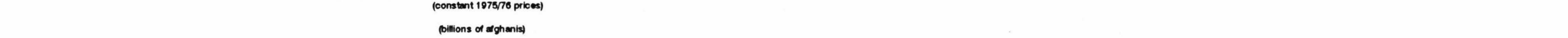 } \\
\hline & Actual & $\begin{array}{l}1068 / 100 \\
\text { cor. } 1000 .\end{array}$ & ads ing. & Actual & $\begin{array}{l}-1960 / 70 \\
\text { cort tro. }\end{array}$ & adj. ig. & Actal & $\begin{array}{l}107071 \\
\text { cor. } 100 .\end{array}$ & ad ing. & Actal & 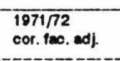 & Actual & $\begin{array}{l}107273 \\
\text { cor. } 1 \text { tro. }\end{array}$ & adj $n_{0}$. & Actral & 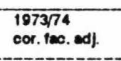 & Actral & 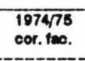 & $\infty<\mathrm{ng}$. & Aotval & $\begin{array}{l}1075 / 6 \\
\text { oor. the. }\end{array}$ & $\infty 1 n_{0}$ & Actual & $\begin{array}{l}197677 \\
\text { oor. tro. }\end{array}$ & $\infty+\infty, n_{0}$. \\
\hline Pivato consumption & 81.51 & 1.00 & 81.51 & 91.32 & 1.00 & 81.32 & 82.14 & 1.00 & 82.14 & 78.63 & 1.0078 & 76.31 & 1.00 & 76.31 & 84.55 & 1.0084 & $\infty 0.00$ & 1.00 & $\infty 0.00$ & 01.67 & 1.00 & 01.67 & 22.2 & 1.00 & 02.2 \\
\hline Govenmment consumption & 3.42 & 1.00 & 3.42 & 3.87 & 1.00 & 3.67 & 3.83 & 1.00 & 3.83 & 3.85 & 1.003 & 4.00 & 1.00 & 4.00 & 4.32 & 1.004 & 4.38 & 1.00 & 4.30 & 0.13 & 1.00 & 0.13 & 6.35 & 1.00 & 6.35 \\
\hline $\begin{array}{l}\text { Gross investment } \\
\text { Private } \\
\text { Public }\end{array}$ & $\begin{array}{l}2.86 \\
4.00\end{array}$ & $\begin{array}{l}1.00 \\
1.00\end{array}$ & $\begin{array}{l}2.86 \\
4.00\end{array}$ & $\begin{array}{l}3.00 \\
4.50\end{array}$ & $\begin{array}{l}1.00 \\
1.00\end{array}$ & $4.3^{3}$ & $\begin{array}{l}3.00 \\
4.50\end{array}$ & $\begin{array}{l}1.00 \\
1.00\end{array}$ & 4.3 & $\begin{array}{l}3.00 \\
5.00\end{array}$ & $\begin{array}{ll}1.00 & 3 \\
1.00 & 3\end{array}$ & $\begin{array}{l}2.73 \\
.2 .20\end{array}$ & $\begin{array}{l}1.00 \\
1.00\end{array}$ & $\begin{array}{l}2.73 \\
4.20\end{array}$ & $\begin{array}{l}3.00 \\
4.00\end{array}$ & $\begin{array}{ll}1.00 & 3 \\
1.00 & 4\end{array}$ & $\begin{array}{l}3.50 \\
5.50\end{array}$ & $\begin{array}{l}1.00 \\
1.00\end{array}$ & $\begin{array}{l}3.5 \\
8.5\end{array}$ & $\begin{array}{l}4.09 \\
6.40\end{array}$ & $\begin{array}{l}1.00 \\
1.00\end{array}$ & $\begin{array}{l}4.03 \\
0.4\end{array}$ & 11.45 & $\begin{array}{l}1.00 \\
1.00\end{array}$ & 11.45 \\
\hline $\begin{array}{l}\text { Export of goods } \\
\text { Less import of goods }\end{array}$ & $\begin{array}{l}4.12 \\
7.03\end{array}$ & $\begin{array}{l}1.00 \\
1.00\end{array}$ & $\begin{array}{l}4.12 \\
7.03\end{array}$ & $\begin{array}{l}4.49 \\
0.05\end{array}$ & $\begin{array}{l}1.00 \\
1.00\end{array}$ & $\begin{array}{l}4.49 \\
6.05\end{array}$ & $\begin{array}{l}4.05 \\
6.08\end{array}$ & $\begin{array}{l}1.00 \\
1.00\end{array}$ & $\begin{array}{l}4.05 \\
6.08\end{array}$ & $\begin{array}{l}5.99 \\
8.90\end{array}$ & $\begin{array}{ll}1.005 \\
1.00 & 8\end{array}$ & $\begin{array}{l}7.10 \\
8.63\end{array}$ & $\begin{array}{l}1.00 \\
1.00\end{array}$ & $\begin{array}{l}7.1 \\
8.63\end{array}$ & $\begin{array}{l}9.00 \\
0.10\end{array}$ & $\begin{array}{ll}1.00 \% \\
1.00 \%\end{array}$ & $\begin{array}{l}12.04 \\
13.40\end{array}$ & $\begin{array}{l}1.00 \\
1.00\end{array}$ & $\begin{array}{l}12.04 \\
13.46\end{array}$ & $\begin{array}{l}13.42 \\
15.20\end{array}$ & $\begin{array}{l}1.00 \\
1.00\end{array}$ & $\begin{array}{l}13.42 \\
18.2\end{array}$ & $\begin{array}{l}17.67 \\
18.67\end{array}$ & $\begin{array}{l}1.00 \\
1.00\end{array}$ & $\begin{array}{l}\begin{array}{l}17.67 \\
18.67\end{array} \\
\end{array}$ \\
\hline $\begin{array}{l}\text { Not income trom non - tractor } \\
\text { services }\end{array}$ & 0.04 & 1.00 & 0.04 & 0.04 & 1.00 & 0.04 & 0.19 & 1.00 & 0.19 & 0.25 & 1.000. & 0.28 & 1.00 & 0.28 & 0.21 & 1.000 & 0.16 & 1.00 & 0.16 & 0.05 & 1.00 & 0.05 & 0.12 & 1.00 & 0.12 \\
\hline $\begin{array}{l}\text { Totad expenditure on GDP } \\
\text { at market pricos }\end{array}$ & 80.01 & & 89.01 & $\$ 0.37$ & & 90.37 & 92.23 & & 92.23 & 87.82 & $\ldots$ & 80.17 & & 86.17 & 95.98 & - & 109.41 & & 100.41 & 108.50 & & 108.50 & 113.22 & & 113.22 \\
\hline
\end{tabular}

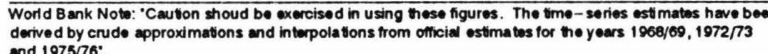

and 1975/76".

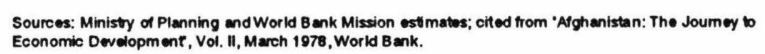


Table A II-6. Official GOA Total National Product, Material Expenditure, and National Income (constant $1975 / 76$ prices)

(billions of afghanis)

\begin{tabular}{|c|c|c|c|c|c|c|c|c|c|}
\hline & \multicolumn{3}{|c|}{$1968 / 69$} & \multicolumn{3}{|c|}{$1972 / 73$} & \multicolumn{3}{|c|}{$1975 / 76$} \\
\hline & $\begin{array}{l}\text { Total } \\
\text { Prod. }\end{array}$ & $\begin{array}{c}\text { Material } \\
\text { Expenditures }\end{array}$ & $\begin{array}{c}\text { Net Prod. } \\
\text { (nat. revenue) }\end{array}$ & $\begin{array}{l}\text { Total } \\
\text { Prod. }\end{array}$ & $\begin{array}{c}\text { Material } \\
\text { Expenditures }\end{array}$ & $\begin{array}{c}\text { Net Prod. } \\
\text { (nat. revenue) }\end{array}$ & $\begin{array}{l}\text { Total } \\
\text { Prod. }\end{array}$ & $\begin{array}{c}\text { Material } \\
\text { Expenditures }\end{array}$ & $\begin{array}{c}\text { Net Prod. } \\
\text { (nat. revenue) }\end{array}$ \\
\hline Industries excl. handicrafts & 4.61 & 3.06 & 1.55 & 7.46 & 5.09 & 2.37 & 11.06 & 7.66 & 3.4 \\
\hline Handicrafts & 10.77 & 8.19 & 2.58 & 12.21 & 9.28 & 2.93 & 12.84 & 9.76 & 3.08 \\
\hline Agriculture \& forestry & 66.77 & 15.74 & 51.03 & 57.73 & 14.35 & 43.38 & 71.44 & 17.63 & 53.81 \\
\hline Construction & 3.36 & 1.93 & 1.43 & 3.37 & 1.98 & 1.39 & 5.44 & 3.27 & 2.17 \\
\hline Transport \& communications & 2.41 & 0.89 & 1.52 & 3.19 & 1.16 & 2.03 & 3.66 & 1.45 & 2.21 \\
\hline Food supply \& dist. (? commerce) & 9.73 & 2.36 & 7.37 & 10.95 & 2.69 & 8.26 & 14.48 & 3.62 & 10.86 \\
\hline Other sectors & 1.46 & 0.48 & 0.98 & 1.66 & 0.61 & 1.05 & 1.78 & 0.69 & 1.09 \\
\hline Total production sectors & 99.11 & 32.65 & 66.46 & 96.57 & 35.16 & 61.41 & 120.7 & 44.08 & 76.62 \\
\hline Imports - Exports & 0.84 & -- & 0.84 & 0.22 & -- & 0.22 & 1.15 & -- & 1.15 \\
\hline Net foreign trade, aid \& credits & 2.69 & -- & 2.69 & 2.12 & -- & 2.12 & 3.18 & -- & 3.18 \\
\hline Total & 102.64 & 32.65 & 69.99 & 98.91 & 35.16 & 63.75 & 125.03 & 44.08 & 80.95 \\
\hline
\end{tabular}

Source: CSO, Statistical Information of Afghanistan (1975-76), p. 102, and Ministry of Planning; cited from "Afghanistan: The Journey to Economic Development", Vol. II, March 1978, World Bank. 
Table A II-7. GNP at Market Prices

(In constant 1975/76 prices)

(billions of afghanis)

\begin{tabular}{|c|c|c|c|}
\hline & $1968 / 69$ & $1972 / 73$ & $1975 / 76$ \\
\hline Total Net Material Output (value added) & 56.59 & 50.07 & 62.46 \\
\hline Trade and transport & 9.87 & 11.34 & 14.17 \\
\hline $\begin{array}{l}\text { Services } \\
\text { Private [a] } \\
\text { Public [b] }\end{array}$ & $\begin{array}{l}3.32 \\
2.56\end{array}$ & $\begin{array}{l}3.07 \\
3.07\end{array}$ & $\begin{array}{l}3.81 \\
4.65\end{array}$ \\
\hline Housing $[c]$ & 4.33 & 4.15 & 5.00 \\
\hline National income & 76.67 & 71.70 & 90.09 \\
\hline Depreciation [d] & 1.53 & 1.47 & 1.94 \\
\hline Factor income (-) [e] & 0.06 & 0.10 & 0.08 \\
\hline GDP at factor cost & 78.14 & 73.07 & 91.95 \\
\hline Indirect taxes & 2.92 & 3.84 & 6.01 \\
\hline Subsidy (-) & 0.37 & 0.70 & 1.90 \\
\hline GNP at market prices & 80.69 & 76.21 & 96.06 \\
\hline
\end{tabular}

[b] $63 \%$ of gov't ordinary budget and $25 \%$ of local financing of development budget expenditures.

[c] $6 \%$ of total material products and services.

[d] Average $2 \%$ of net national product at factor cost calculated on the basis of separate depreciation coefficients in various sectors.

[e] Interest on public debt is used as proxy factor income assuming that the other inflows and outflows are balanced.

Source: Ministry of Planning; cited from "Afghanistan: The Journey to Economic Development", Vol. II, March 1978, World Bank. 
Table A II-8. RRNA Adjustments of GDP by Sector at Constant 1978/79 Prices (millions of afghanis, unless otherwise indicated)

\begin{tabular}{|c|c|c|c|c|c|c|c|c|c|c|c|c|}
\hline & $\begin{array}{l}\text { 1978-79 } \\
\text { Actual }\end{array}$ & $\begin{array}{l}\text { Corr. } \\
\text { Factor }\end{array}$ & $\begin{array}{l}\text { Adjusted } \\
\text { Figure }\end{array}$ & $\begin{array}{l}\text { 1984-85 } \\
\text { Actual }\end{array}$ & $\begin{array}{l}\text { Corr. } \\
\text { Factor }\end{array}$ & $\begin{array}{l}\text { Adjusted } \\
\text { Figure }\end{array}$ & $\begin{array}{l}\text { 1985-86 } \\
\text { Actual }\end{array}$ & $\begin{array}{l}\text { Corr. } \\
\text { Factor }\end{array}$ & $\begin{array}{l}\text { Adjusted } \\
\text { Figure }\end{array}$ & $\begin{array}{l}1986-87 \\
\text { Actual }\end{array}$ & $\begin{array}{l}\text { Corr. } \\
\text { Factor }\end{array}$ & $\begin{array}{l}\text { Adjusted } \\
\text { Figure }\end{array}$ \\
\hline \multicolumn{13}{|l|}{ 1. Agriculture, Livestock } \\
\hline \& Forestry & 61,900 & & 58,392 & 50,758 & & 29,381 & 49,520 & & 26,296 & 49,000 & & 22,257 \\
\hline Crops & 42,158 & 0.92 & 38,650 & 34,569 & 0.38 & 13,192 & 33,726 & 0.31 & 10,502 & 33,350 & 0.20 & 6,607 \\
\hline Livestock & 19,441 & 1.00 & 19,441 & 15,942 & 1.00 & 15,942 & 15,553 & 1.00 & 15,553 & 15,415 & 1.00 & 15,415 \\
\hline Forestry & 301 & 1.00 & 301 & 247 & 1.00 & 247 & 241 & 1.00 & 241 & 235 & 1.00 & 235 \\
\hline \multicolumn{13}{|l|}{ 2. Mining Industry } \\
\hline \& Energy & 14,400 & & 14,400 & 13,428 & & 13,428 & 14,321 & & 14,321 & 14,651 & & 14,651 \\
\hline Mining & 1,550 & 1.00 & 1,550 & 1,761 & 1.00 & 1,761 & 1,688 & 1.00 & 1,688 & 1,525 & 1.00 & 1,525 \\
\hline Industry & 3,909 & 1.00 & 3,909 & 4,029 & 1.00 & 4,029 & 5,137 & 1.00 & 5,137 & 5,699 & 1.00 & 5,699 \\
\hline Electricity & 376 & 1.00 & 376 & 438 & 1.00 & 438 & 473 & 1.00 & 473 & 527 & 1.00 & 527 \\
\hline Handicrafts & 8,565 & 1.00 & 8,565 & 7,200 & 1.00 & 7,200 & 7,023 & 1.00 & 7,023 & 6,900 & 1.00 & 6,900 \\
\hline 3. Construction & 4,300 & 1.00 & 4,300 & 3,900 & 1.00 & 3,900 & 4,000 & 1.00 & 4,000 & 4,800 & 1.00 & 4,800 \\
\hline \multicolumn{13}{|l|}{ 4. Transport } \\
\hline \& Communications & 3,500 & 1.00 & 3,500 & 3,700 & 1.00 & 3,700 & 2,700 & 1.00 & 2,700 & 2,900 & 1.00 & 2,900 \\
\hline 5. Trade \& Distibution & 9,700 & 1.00 & 9,700 & 9,100 & 1.00 & 9,100 & 9,000 & 1.00 & 9,000 & 9,100 & 1.00 & 9,100 \\
\hline 6. Others & 1,700 & 1.00 & 1,700 & 1,800 & 1.00 & 1,800 & 1,700 & 1.00 & 1,700 & 1,700 & 1.00 & 1,700 \\
\hline 7. Total Production & 95,500 & & 91,992 & 82,686 & & 61,309 & 81,241 & & 58,017 & 82,151 & & 55,408 \\
\hline 8. Services & 9,275 & & 9,275 & 12,575 & & 12,575 & 12,138 & & 12,138 & 13,242 & & 13,242 \\
\hline Private $(5 \%$ of 7$)$ & 4,775 & 1.00 & 4,775 & 4,150 & 1.00 & 4,150 & 4,050 & 1.00 & 4,050 & 4,102 & 1.00 & 4,102 \\
\hline Public & 4,500 & 1.00 & 4,500 & 8,425 & 1.00 & 8,425 & 8,088 & 1.00 & 8,088 & 9,140 & 1.00 & 9,140 \\
\hline 9. Housing $(6 \%$ of 7$)$ & 5,730 & 1.00 & 5,730 & 4,980 & 1.00 & 4,980 & 4,860 & 1.00 & 4,860 & 4,922 & 1.00 & 4,922 \\
\hline \multicolumn{13}{|l|}{ 10. Total Net Domestic } \\
\hline Product at factor cost & 110,505 & & 106,997 & 100,241 & & 78,864 & 98,239 & & 75,015 & 100,315 & & 73,572 \\
\hline 11. Depreciation & 6,700 & 1.00 & 6,700 & 7,300 & 1.00 & 7,300 & 7,400 & 1.00 & 7,400 & 7,400 & 1.00 & 7,400 \\
\hline \multicolumn{13}{|l|}{ 12. Total Gross Domestic } \\
\hline & 111,205 & & 110,698 & 107,541 & & 86,164 & 105,639 & & 82,415 & $108, \pi 15$ & & 00,512 \\
\hline
\end{tabular}

RRNA Note 1. Adjusted GDP figures do not correspond to those which appear in Table A I-1 due to rounding.

RRNA Note 2. Only agricultural crop production figures are adjusted since we have no information to suggest either growth or decline in any of the other principal sectors.

UNDP Note 1. With the exception of GDP of Construction and Transport and Communications, the figures presented in this table differ from official data as they have been adjusted to reflect the depressed situation of the economy over the period. Official figures are given in Annex Table 1 of the UNDP Discussion Paper on Afghanistan of April 1988.

UNDP Note 2. The Government methodology does not provide figures on the GDP of the services sector. In order to give a complete picture of GDP at factor cost above these flows were added using the methodology presented in the table. The Government GDP, mainly wages and salaries, has been expressed at constant prices using the consumer price index.

Sources: Original data from Central Statistical Office; cited from the UNDP Discussion Paper on Afghanistan of April 1988. 
Table A II-9. RRNA Adjustments of Estimated Production of Principal Crops

(thousands of metric tons)

\begin{tabular}{|c|c|c|c|c|c|c|c|c|c|c|c|c|c|}
\hline & $1977 / 78$ & & $1978 / 79$ & & & $1979 / 80$ & & & $1980 / 81$ & & & $1981 / 82$ & \\
\hline & Official & Official & Rev.1 & Rev.2 & Official & Rev.1 & Rev.2 & Official & Rev.1 & Rev.2 & Official & Rev.1 & Rev.2 \\
\hline Correction Factor & $100.00 \%$ & $100.00 \%$ & $6.20 \%$ & $7.36 \%$ & $100.00 \%$ & $12.30 \%$ & $14.72 \%$ & $100.00 \%$ & $18.40 \%$ & $22.08 \%$ & $100.00 \%$ & $24.50 \%$ & $29.44 \%$ \\
\hline Foodgrains & 4,112 & 4,382 & 4,110 & 4,059 & 4,180 & 3,666 & 3,565 & 4,370 & 3,566 & 3,405 & 4,491 & 3,391 & 3,169 \\
\hline Wheat & 2,652 & 2,813 & 2,639 & 2,606 & 2,663 & 2,335 & 2,271 & 2,750 & 2,244 & 2,143 & 2,850 & 2,152 & 2,011 \\
\hline Barley & 300 & 325 & 305 & 301 & 318 & 279 & 271 & 321 & 262 & 250 & 330 & 249 & 233 \\
\hline Corn & 760 & 780 & 732 & 723 & 760 & 667 & 648 & 797 & 650 & 621 & 798 & 602 & 563 \\
\hline Rice & 400 & 428 & 401 & 396 & 439 & 385 & 374 & 461 & 376 & 359 & 475 & 359 & 335 \\
\hline Other foodgrains & -- & 36 & 34 & 33 & -- & -- & -- & 41 & 33 & 32 & 38 & 29 & 27 \\
\hline Fruits and vegetables & 1,352 & 1,590 & 1,491 & 1,473 & 1,640 & 1,438 & 1,399 & 1,719 & 1,403 & 1,339 & 1,778 & 1,342 & 1,255 \\
\hline Fruits & 692 & 824 & 773 & 763 & 836 & 733 & 713 & 891 & 727 & 694 & 913 & 689 & 644 \\
\hline Vegetables & 660 & 766 & 719 & 710 & 804 & 705 & 686 & 828 & 676 & 645 & 865 & 653 & 610 \\
\hline Industial crops & 334 & 304 & 285 & 282 & 281 & 246 & 240 & 213 & 174 & 166 & 194 & 146 & 137 \\
\hline Cotton & 137 & 132 & 124 & 122 & 105 & 92 & 90 & 65 & 53 & 51 & 60 & 45 & 42 \\
\hline Sugar beets & 97 & 73 & 68 & 68 & 70 & 61 & 60 & 35 & 29 & 27 & 20 & 15 & 14 \\
\hline Sugarcane & 64 & 64 & 60 & 59 & 64 & 56 & 55 & 70 & 57 & 55 & 70 & 53 & 49 \\
\hline Oilseeds & 36 & 35 & 33 & 32 & 42 & 37 & 36 & 43 & 35 & 34 & 44 & 33 & 31 \\
\hline
\end{tabular}

Note: These figures have been revised downward based on estimates of the decline in agricultural production made by the Agricultural Survey

of Afghanistan, under the Swedish Committee for Afghanistan. Revision 1 is based on published estimates of the decline in agricultural

production. Revision 2 is a further $25 \%$ downward adjustment of published estimates based on the verbally expressed opinion of Swedish

Committee officials and other individuals involved with the Agricultural Survey of Afghanistan.

Sources: "Afghanistan: Recent Economic Developments", IMF, 1983, 1984, 1985, 1986. From the Government of

Afghanistan, Central Statistical Office, and the Ministry of Agriculture and Land Reform; the Swedish Committee for Afghanistan. 
Table A II-9 (continued). RRNA Adjustments of Estimated Production of Principal Crops (thousands of metric tons)

\begin{tabular}{|c|c|c|c|c|c|c|c|c|c|c|c|c|c|c|}
\hline & $1982 / 83$ & & & $1983 / 84$ & & & $1984 / 85$ & & & $1985 / 86$ & & & $\begin{array}{r}1986 / 87 \\
\text { Est. }\end{array}$ & \\
\hline Official & Rev.1 & Rev.2 & Official & Rev.1 & Rev. 2 & Official & Rev.1 & Rev.2 & Official & Rev.1 & Rev.2 & Official & Rev.1 & Rev. 2 \\
\hline $100.00 \%$ & $30.60 \%$ & $36.81 \%$ & $100.00 \%$ & $36.70 \%$ & $44.17 \%$ & $100.00 \%$ & $42.80 \%$ & $51.53 \%$ & $100.00 \%$ & $48.90 \%$ & $58.89 \%$ & $100.00 \%$ & $55.00 \%$ & $66.25 \%$ \\
\hline $4,500$. & 3,123 & 2,844 & 4,590 & 2,905 & 2,563 & 4,507 & 2,578 & 2,185 & 4,500 & 2,300 & 1,850 & 4,408 & 1,984 & 1,488 \\
\hline 2,860 & 1,985 & 1,807 & 2,927 & 1,853 & 1,634 & 2,860 & 1,636 & 1,386 & 2,850 & 1,456 & 1,172 & 2,750 & 1,238 & 928 \\
\hline 329 & 228 & 208 & 336 & 213 & 188 & 332 & 190 & 161 & 333 & 170 & 137 & 330 & 149 & 111 \\
\hline 800 & 555 & 506 & 806 & 510 & 450 & 798 & 456 & 387 & 799 & 408 & 328 & 810 & 365 & 273 \\
\hline 473 & 328 & 299 & 480 & 304 & 268 & 479 & 274 & 232 & 480 & 245 & 197 & 480 & 216 & 162 \\
\hline 38 & 26 & 24 & 41 & 26 & 23 & 38 & 22 & 18 & 38 & 19 & 16 & 38 & 17 & 13 \\
\hline 1,832 & 1,271 & 1,158 & 1,860 & 1,177 & 1,038 & 1,862 & 1,065 & 903 & 1,847 & 944 & 759 & 1,920 & 864 & 648 \\
\hline 920 & 638 & 581 & 930 & 589 & 519 & 930 & 532 & 451 & 927 & 474 & 381 & 950 & 428 & 321 \\
\hline 912 & 633 & 576 & 930 & 589 & 519 & 932 & 533 & 452 & 920 & 470 & 378 & 970 & 437 & 327 \\
\hline 182 & 126 & 115 & 184 & 116 & 103 & 212 & 121 & 103 & 198 & 101 & 81 & 203 & 91 & 69 \\
\hline 45 & 31 & 28 & 50 & 32 & 28 & 68 & 39 & 33 & 75 & 38 & 31 & 75 & 34 & 25 \\
\hline 20 & 14 & 13 & 16 & 10 & 9 & 26 & 15 & 13 & 5 & 3 & 2 & 8 & 4 & 3 \\
\hline 72 & 50 & 46 & 73 & 46 & 41 & 73 & 42 & 35 & 73 & 37 & 30 & 75 & 34 & 25 \\
\hline 45 & 31 & 28 & 45 & 28 & 25 & 45 & 26 & 22 & 45 & 23 & 18 & 45 & 20 & 15 \\
\hline
\end{tabular}


Table A II-11. GOA Estimates of Production of Principal Crops

(thousands of metric tons)

\begin{tabular}{|c|c|c|c|c|c|c|c|c|c|c|}
\hline & $1977 / 78$ & $1978 / 79$ & $1979 / 80$ & $1980 / 81$ & $1981 / 82$ & $1982 / 83$ & $1983 / 84$ & $1984 / 85$ & $1985 / 86$ & $\begin{array}{r}1986 / 87 \\
\text { Est. }\end{array}$ \\
\hline Foodgrains & 4,112 & 4,346 & 4,180 & 4,370 & 4,491 & 4,500 & 4,590 & 4,507 & 4,500 & 4,408 \\
\hline Wheat & 2,652 & 2,813 & 2,663 & 2,750 & 2,850 & 2,860 & 2,927 & 2,860 & 2,850 & 2,750 \\
\hline Barley & 300 & 325 & 318 & 321 & 330 & 329 & 336 & 332 & 333 & 330 \\
\hline Corn & 760 & 780 & 760 & 797 & 798 & 800 & 806 & 798 & 799 & 810 \\
\hline Rice & 400 & 428 & 439 & 461 & 475 & 473 & 480 & 479 & 480 & 480 \\
\hline Other foodgrains & -- & -- & -- & 41 & 38 & 38 & 41 & 38 & 38 & 38 \\
\hline Fruits and vegetables & 1,352 & 1,590 & 1,640 & 1,719 & 1,778 & 1,832 & 1,860 & 1,862 & 1,847 & 1,920 \\
\hline Fruits & 692 & 824 & 836 & 891 & 913 & 920 & 930 & 930 & 927 & 950 \\
\hline Vegetables & 660 & 766 & 804 & 828 & 865 & 912 & 930 & 932 & 920 & 970 \\
\hline Industrial crops & 334 & 304 & 281 & 213 & 194 & 182 & 184 & 212 & 198 & 203 \\
\hline Cotton & 137 & 132 & 105 & 65 & 60 & 45 & 50 & 68 & 75 & 75 \\
\hline Sugar beets & 97 & 73 & 70 & 35 & 20 & 20 & 16 & 26 & 5 & 8 \\
\hline Sugarcane & 64 & 64 & 64 & 70 & 70 & 72 & 73 & 73 & 73 & 75 \\
\hline Oilseeds & 36 & 35 & 42 & 43 & 44 & 45 & 45 & 45 & 45 & 45 \\
\hline
\end{tabular}

Sources: GOA Central Statistical Office, and the Ministry of Agriculture and Land Reform; cited in

"Afghanistan: Recent Economic Developments", 1983, 1984, 1985, 1986, IMF. 
Table A II-12. Trends in Production of Principal Crops

(thousands of tons)

\begin{tabular}{|c|c|c|c|c|c|c|c|}
\hline Crop & $1970 / 71$ & $1971 / 72$ & $1972 / 73$ & $1973 / 74$ & $1974 / 75$ & $1975 / 76$ & $\begin{array}{r}\text { Est. } \\
1976 / 77\end{array}$ \\
\hline Wheat & 2,081 & 1,915 & 2,450 & 2,700 & 2,750 & 2,850 & 2,936 \\
\hline Barley & 370 & 355 & 350 & 360 & 380 & 384 & 400 \\
\hline Maize & 667 & 670 & 720 & 760 & 770 & 780 & 800 \\
\hline Rice & 366 & 350 & 400 & 420 & 420 & 435 & 448 \\
\hline $\begin{array}{l}\text { Cotton } \\
\text { (unginned) }\end{array}$ & 76 & 63 & 58 & 108 & 145 & 160 & 159 \\
\hline Sugarbeet & 62 & 60 & 63 & 63.6 & 66.6 & 100 & 91 \\
\hline Sugarcane & 55 & 50 & 17.3 & 22.2 & 51.6 & 60 & 68 \\
\hline Oilseeds & 35 & 28 & 34 & 38 & 40 & 40 & 62 \\
\hline Fruits & 809 & 650 & 820 & 840 & 865 & 880 & 900 \\
\hline Vegetables & 626 & 725 & 658 & 680 & 700 & 720 & 918 \\
\hline
\end{tabular}

Source: GOA Central Statistical Office; cited from "Afghanistan: The Journey to Economic Development", Vol. II, March 1978, World Bank. 
Table A II-13. Pre-war Area of Agricultural and Horticultural Crop Production

(thousands of hectares)

\begin{tabular}{|c|c|c|c|}
\hline Provinces & $\begin{array}{l}\text { Total } \\
\text { Irrigated } \\
\text { Area }\end{array}$ & $\begin{array}{l}\text { Total Area of } \\
\text { Horticultural } \\
\text { Crops }\end{array}$ & $\begin{array}{l}\text { Horticultural Crops as \% } \\
\text { of Total Irrigated } \\
\text { Area }\end{array}$ \\
\hline Kabul & 70 & 10.6 & 15.1 \\
\hline Kapisa/Parvan & 102 & 22.5 & 22.9 \\
\hline Vardak & 32 & 4.0 & 12.5 \\
\hline Lowgar & 41 & 5.6 & 13.6 \\
\hline Ghazni & 158 & 10.8 & 6.8 \\
\hline Paktia & 87 & 6.3 & 7.2 \\
\hline Nangarhar/Konarha & 135 & 16.1 & 11.9 \\
\hline Laghman & 46 & 6.4 & 13.9 \\
\hline Bamian & 30 & 4.3 & 14.0 \\
\hline Regional Avg. & 77.9 & 9.6 & 13.1 \\
\hline Country Avg. & 149 & 8.9 & 6.0 \\
\hline
\end{tabular}

Source: "Afghanistan: The Journey to Economic Development", Vol. II, March 1978,

World Bank. 
Table A II-14. Pre-war Agricultural

Production in Eastern Provinces

(Kabuli seers)

\begin{tabular}{|c|c|c|c|c|c|}
\hline Province & $\begin{array}{l}\text { Total Grain } \\
\text { Output }\end{array}$ & $\begin{array}{l}\text { Output } \\
\text { per } \\
\text { Capita }\end{array}$ & $\begin{array}{l}\text { Output } \\
\text { per Ha of } \\
\text { Cult. Land }\end{array}$ & $\begin{array}{l}\text { Vegetable } \\
\text { Production }\end{array}$ & $\begin{array}{c}\text { Fruit } \\
\text { Production }\end{array}$ \\
\hline Kabul & $10,282,200$ & 16.1 & 152.0 & $9,537,750$ & $3,460,000$ \\
\hline Kapisa & $6,373,200$ & 13.8 & 157.6 & $1,264,410$ & $4,100,880$ \\
\hline Parvan & $7,321,800$ & 14.8 & 150.2 & $5,526,000$ & $9,924,000$ \\
\hline Vardak & $4,534,500$ & 11.8 & 165.4 & $3,015,540$ & 899,760 \\
\hline Ghazni & $22,673,900$ & 30.6 & 133.9 & $4,135,130$ & $5,094,400$ \\
\hline Paktia & $11,094,000$ & 15.7 & 126.5 & $2,286,270$ & $2,888,000$ \\
\hline Nangarhar & $16,073,600$ & 25.1 & 261.5 & $5,623,020$ & $3,492,480$ \\
\hline Laghman & $9,164,600$ & 56.6 & 365.4 & $4,239,000$ & $2,259,840$ \\
\hline Konarha & $5,312,400$ & 20.3 & 201.0 & $1,557,000$ & $1,626,240$ \\
\hline Bamian & $4,474,500$ & 16.3 & 149.6 & $1,433,700$ & 865,600 \\
\hline Total & $97,304,700$ & & & $38,617,820$ & $34,611,200$ \\
\hline
\end{tabular}

Note: One seer is equal to approximately 7.07 kilograms. Grain per capita is a measure of output per member of the agricultural population, excluding landlords. It is a rough measure of output per worker.

Source: Kabul, Ludwig Adamec, provincial entries; cited from the Orkand Report. 
Table A II-15. Wheat Production in

Eastern Provinces

\begin{tabular}{|c|c|c|c|c|c|c|}
\hline \multirow[b]{2}{*}{ Province } & \multicolumn{2}{|c|}{ Average Yield (kg/ha) } & & \multicolumn{2}{|c|}{ Fertilizer Use (kg/ha) } & \multirow[b]{2}{*}{$\%$ Change } \\
\hline & Pre-War & $1983 / 84$ & $\%$ Change & Pre-War & $1983 / 84$ & \\
\hline Kabul & 840 & 504 & $-40 \%$ & 86.8 & 12.3 & $-86 \%$ \\
\hline Kapisa & 770 & 490 & $-36 \%$ & 58.8 & 11.2 & $-81 \%$ \\
\hline Parvan & 840 & 490 & $-42 \%$ & 70.0 & -- & -- \\
\hline Vardak & 980 & 532 & $-46 \%$ & 49.0 & 28.0 & $-43 \%$ \\
\hline Lowgar & 980 & 532 & $-46 \%$ & 91.0 & 29.4 & $-68 \%$ \\
\hline Ghazni & 840 & 392 & $-53 \%$ & 67.2 & 25.2 & $-63 \%$ \\
\hline Paktia & 812 & 322 & $-60 \%$ & 42.0 & 33.6 & $-20 \%$ \\
\hline Nangarhar & 1,120 & 504 & $-55 \%$ & 79.8 & -- & -- \\
\hline Laghman & 1,190 & 588 & $-51 \%$ & 98.0 & 32.2 & $-67 \%$ \\
\hline Konarha & 1,372 & 504 & $-63 \%$ & 51.8 & 35.0 & $-32 \%$ \\
\hline Bamian & 630 & 448 & $-29 \%$ & 21.0 & 4.2 & $-80 \%$ \\
\hline Country Avg. & 811 & 464 & $-43 \%$ & 62.4 & 19.7 & $-68 \%$ \\
\hline
\end{tabular}

Source: "Study on Agriculture in Afghanistan (1984)," by Mohammad

Ebrahim Rashid, Appendix Table IV; cited from the Orkand Report. 
Table A II-16. Pre-war Area of Cultivation in Eastern Provinces

\begin{tabular}{|c|c|c|c|c|c|}
\hline \multirow[b]{2}{*}{ Province } & \multirow[b]{2}{*}{$\begin{array}{l}\text { Number of } \\
\text { Villages }\end{array}$} & \multicolumn{4}{|c|}{ Area Under Cultivation [a] } \\
\hline & & $\begin{array}{l}\text { Agricultural - } \\
\text { Population }\end{array}$ & Irrigated & Rainfed & Total \\
\hline Kabul & 384 & 639,800 & 287,980 & 50,310 & 338,290 \\
\hline Kapisa & 381 & 460,810 & 199,710 & 2,480 & 202,190 \\
\hline Parvan & 345 & 493,720 & 175,700 & 67,960 & 243,660 \\
\hline Vardak & 183 & 382,790 & 128,010 & 9,070 & 137,080 \\
\hline Lowgar & 265 & 237,360 & 133,260 & 86,630 & 219,890 \\
\hline Hgazni & 2,039 & 760,210 & 587,600 & 259,450 & 847,050 \\
\hline Paktia & 1,033 & 704,690 & 281,700 & 156,860 & 438,560 \\
\hline Nangarhar & 531 & 640,900 & 221,730 & 90,650 & 312,380 \\
\hline Laghman & 222 & 161,910 & 117,910 & 7,500 & 125,410 \\
\hline Konarha & 265 & 261,670 & 116,570 & 15,570 & 132,140 \\
\hline Bamian & 285 & 285,040 & 115,790 & 33,750 & 149,540 \\
\hline Total Central & 5,933 & $5,028,900$ & $2,365,960$ & 780,230 & $3,146,190$ \\
\hline Total Country & 15,270 & $12,759,620$ & $11,926,130$ & $10,819,270$ & $22,745,400$ \\
\hline
\end{tabular}

[a] Jeribs. One hectare equals approximately 5 jeribs.

Source: Central Statistical Office, 1977, Government of Afghanistan; cited from the Orkand Report. 
Table A II-17. Pre-war Number and Type "

of Animals in Eastern Provinces

\begin{tabular}{lrrrrrr}
\hline Province & Sheep & Goats & Cattle & Horses & Donkeys & Mules \\
\hline Kabul & 64,080 & 9,160 & 48,400 & 1,240 & 37,540 & \\
Kapisa & 86,380 & 54,780 & 56,620 & 1,770 & 30,530 & 1,210 \\
Parvan & 77,190 & 68,020 & 60,910 & 490 & 19,010 & \\
Vardak & 207,350 & 29,600 & 59,920 & -- & 42,560 & 11,030 \\
Lowgar & 37,290 & 3,520 & 31,680 & 1,140 & 33,790 & \\
Ghazni & 887,600 & 48,500 & 302,690 & 2,200 & 84,920 & \\
Paktia & 147,170 & 227,060 & 190,742 & 1,010 & 47,960 & \\
Nangarhar & 61,330 & 43,110 & 148,380 & 770 & 34,780 & \\
Laghman & 48,400 & 55,130 & 35,730 & 1,730 & 23,040 & 1,660 \\
Konarha & 65,870 & 92,290 & 60,450 & 1,190 & 16,930 & \\
Bamian & 255,380 & 49,040 & 144,560 & 6,610 & 38,550 & \\
& $-1,-1,-0 .-10$ \\
Total & $1,938,040$ & 680,210 & $1,140,082$ & 18,150 & 409,610 & 13,900
\end{tabular}

Note: There were no Qarakul sheep reported in any of these provinces.

Nangarhar was, in addition, reported to have had 16,020 mules.

Source: Central Statistical Office, 1977, Government of Afghanistan; cited from the Orkand Report. 
Table A II-18. Wheat Production in Northern Afghanistan

\begin{tabular}{|c|c|c|c|c|c|c|}
\hline \multirow[b]{2}{*}{ Province } & \multicolumn{3}{|c|}{ Average Yield (kg/ha) } & \multicolumn{3}{|c|}{ Fertilizer Use (kg/ha) } \\
\hline & Pre-War & $1983 / 84$ & $\%$ Change & Pre-War & $1983 / 84$ & $\%$ Change \\
\hline Badakhshan & 490 & 308 & $-37.1 \%$ & 28.0 & & \\
\hline Badghisat & 644 & 378 & $-41.3 \%$ & 30.8 & 7.0 & $-77.3 \%$ \\
\hline Baghlan & 1,260 & 770 & $-38.9 \%$ & 70.0 & 33.6 & $-52.0 \%$ \\
\hline Balkh & 910 & 490 & $-46.2 \%$ & 63.0 & 29.4 & $-53.3 \%$ \\
\hline Faryab & 770 & 448 & $-41.8 \%$ & 56.0 & 16.8 & $-70.0 \%$ \\
\hline Jowzjan & 924 & 560 & $-39.4 \%$ & 49.0 & 28.0 & $-42.9 \%$ \\
\hline Konduz & 1,260 & 630 & $-50.0 \%$ & 91.0 & 35.0 & $-61.5 \%$ \\
\hline Samangan & 910 & 392 & $-56.9 \%$ & 84.0 & 35.0 & $-58.3 \%$ \\
\hline Takhar & 840 & 350 & $-58.3 \%$ & 42.0 & 16.8 & $-60.0 \%$ \\
\hline Country Avg & 811 & 464 & $-42.8 \%$ & 62.4 & 19.7 & $-68.4 \%$ \\
\hline
\end{tabular}

Source: "Study on Agriculture in Afghanistan (1984)," by Mohammad Ebrahim

Rashid, Appendix Table IV; cited from the Orkand Report. 
Table A II-19. Actual and Projected Cotton Production in Northern Provinces

(thousands of hectares)

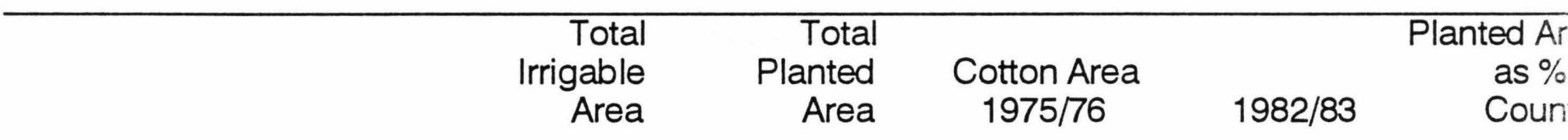

Spinzar Area

\begin{tabular}{|c|c|c|}
\hline Takhar & 232 & 62 \\
\hline Konduz & 286 & 209 \\
\hline Baghlan & 177 & 80 \\
\hline Samangan & 134 & 44 \\
\hline Subtotal & 829 & 395 \\
\hline
\end{tabular}

Balkh Area

\begin{tabular}{|c|c|}
\hline $\begin{array}{l}\text { Balkh } \\
\text { Jowzjan } \\
\text { Faryab }\end{array}$ & $\begin{array}{l}291 \\
251 \\
236\end{array}$ \\
\hline Subtotal & 778 \\
\hline
\end{tabular}

62

209

80

44
45.1

97.1

45.1

97.1

25

24.0

30.0

$24.0 \quad 30.0$

2 .

679

5:

$3,214 \quad 1,358$

10:

Source: "Afghanistan: The Journey to Economic Development", Vol. II, March 1978, World Bank. 
Table A II-20. Pre-war Area of Cultivation in Northern Provinces

\begin{tabular}{|c|c|c|c|c|c|}
\hline \multirow[b]{2}{*}{ Province } & \multirow{2}{*}{$\begin{array}{r}\text { Number of } \\
\text { Villages }\end{array}$} & \multirow[b]{2}{*}{ Ag. Pop. } & \multirow[b]{2}{*}{ Irrigated } & \multicolumn{2}{|c|}{ Area Under Cultivation [a] } \\
\hline & & & & Rainfed & Total \\
\hline Badakhshan & 607 & 398,720 & 308,800 & 600,800 & 909,600 \\
\hline Badghisat & 356 & 295,260 & 166,520 & 603,980 & 770,500 \\
\hline Baghlan & 282 & 466,980 & 400,890 & 768,250 & $1,169,140$ \\
\hline Balkh & 364 & 270,000 & $1,132,500$ & 443,430 & $1,575,930$ \\
\hline Faryab & 456 & 415,650 & 608,030 & 964,270 & $1,572,300$ \\
\hline Jowzjan & 668 & 290,560 & 923,000 & 698,710 & $1,621,710$ \\
\hline Konduz & 293 & 356,630 & $1,047,930$ & 428,570 & $1,476,500$ \\
\hline Samangan & 368 & 222,150 & 221,700 & 729,610 & 951,310 \\
\hline Takhar & 699 & 332,330 & 309,270 & $1,491,480$ & $1,800,750$ \\
\hline Total North & 4,093 & $3,048,280$ & $5,118,640$ & $6,729,100$ & $11,847,740$ \\
\hline Total Country & 15,270 & $12,759,620$ & $11,926,130$ & $10,819,270$ & $22,745,400$ \\
\hline
\end{tabular}

[a] Figures in Jeribs. One hectare is equal to approximately 5 jeribs.

Source: Central Statistical Office, 1977, Government of Afghanistan; cited from the Orkand Report. 
Table A II-21. Pre-war Number and Type of Animals in Northern Provinces

\begin{tabular}{lrrrrrrr}
\hline Province & $\begin{array}{c}\text { Sheep } \\
\text { (Ordinary) }\end{array}$ & (Qarakul) & Goats & Cattle & Horses & Donkeys & Mules \\
\hline Badakhshan & 377,870 & 2,190 & 253,210 & 177,450 & 21,160 & 57,310 & -- \\
Badghisat & $1,393,660$ & 472,750 & 286,550 & 103,500 & 15,790 & 37,950 & 1,210 \\
Baghlan & 621,500 & 28,000 & 186,640 & 116,860 & 25,890 & 45,800 & -- \\
Balkh & 807,550 & $1,393,660$ & 152,440 & 93,340 & 55,960 & 41,770 & 11,030 \\
Faryab & 310,800 & 938,280 & 84,310 & 19,608 & 35,390 & 60,910 & -- \\
Jowzjan & 345,730 & $2,693,440$ & 135,120 & 224,740 & 63,420 & 77,010 & -- \\
Konduz & 318,240 & 247,310 & 73,120 & 179,700 & 18,410 & 30,530 & -- \\
Samangan & 934,830 & 588,510 & 104,030 & 118,300 & 50,160 & 88,350 & -- \\
Takhar & 415,240 & 103,810 & 164,890 & 159,020 & 26,140 & 41,190 & 1,660
\end{tabular}

Source: Central Statistical Office, Government of Afghanistan; cited from the Orkand Report. 
Table A II-22. Pre-war Agricultural Cooperatives by Province

\begin{tabular}{lrrr}
\hline Area & Number & Membership & $\begin{array}{r}\text { Membership as } \\
\text { \% of Country }\end{array}$ \\
\hline Northern Provinces & 39 & 3,894 & 46.7 \\
$\quad$ Baghlan & 7 & 597 & 6.6 \\
Balkh & 17 & 2,187 & 24.0 \\
Jowzjan & 8 & 373 & 8.0 \\
Konduz & 2 & 113 & 1.2 \\
Samangan & 5 & 624 & 6.9 \\
Other Provinces & 34 & 2,646 & 29.0 \\
$\quad$ Helmand & 4 & 627 & 6.9 \\
Herat & 2 & 373 & 4.1 \\
Kandahar & 12 & 628 & 6.9 \\
Lowgar & 7 & 557 & 6.1 \\
Nangarhar & 7 & 386 & 4.2 \\
Parvan & 2 & 75 & 0.8 \\
Capital Area & & & \\
Kabul & 29 & 2,200 & 24.2 \\
Total Country & 102 & 8,740 & 99.9 \\
& & & \\
\hline
\end{tabular}

Source: GOA Ministry of Agriculture; cited from

"Afghanistan: The Journey to Economic Development", Vol.

II, March 1978, World Bank. 
Table A II-23. Pre War Population/Landlord Ratios \& Percent of Cultivated, Irrigated Land in Kabul Province

\begin{tabular}{lrrr}
\hline & $\begin{array}{c}\text { Pop./Landlord } \\
\text { Ratio }\end{array}$ & $\begin{array}{c}\text { \% of District } \\
\text { Under Irrigation }\end{array}$ & $\begin{array}{c}\text { \% of all Irrigated } \\
\text { Land in Province }\end{array}$ \\
\hline Bagrami & 4.2 & 84.1 & 12.8 \\
Paghmani & 18.9 & 89.2 & 10.3 \\
Chahar Dehi & 33.8 & 91.3 & 7.8 \\
Deh Sabz-e-Khas & 10.5 & 74.4 & 13.8 \\
Sarowbi & 5.9 & 74.5 & 2.5 \\
Chakar Dara & 11.7 & 94.4 & 16.0 \\
Qara Bagh & 9.3 & 72.9 & 12.1 \\
Mir Bacheh Kowt & 12.4 & 95.3 & 3.6 \\
(Kohdaman) & & & 8.6 \\
Estalef & 12.0 & 97.8 & 1.1 \\
Kahak-i-Jabar & 24.8 & 70.0 & 7.1 \\
Chahar Asiab & 20.4 & 96.7 & 4.2 \\
Kalakan & 24.0 & 82.5 & 99.9 \\
Provincial Avg. & & & Total = \\
\end{tabular}

Note: Total \% of land irrigated in the province does not equal 100 due to rounding.

Source: Ludwig Adamec, "Kabul and Southeast Afghanistan", (Kabul Section), 1985; cited from the Orkand Report. 
Table A II-24. Basis of UNDP Projection of Essential

Commodity Requirement for 1989/90 - 1991/92

\begin{tabular}{|c|c|c|c|c|c|}
\hline & $1978 / 79$ & $1988 / 89$ & Three year & gram & \\
\hline & Actual & Estimate & $1989 / 90$ & $1990 / 91$ & $1991 / 92$ \\
\hline $\begin{array}{l}\text { Food Requirement } \\
\text { Food Grains (000 tons) } \\
\text { Domestic Production }\end{array}$ & & & & & \\
\hline Wheat & 2,813 & 2,380 & 2,510 & 2,650 & 2,800 \\
\hline Rice & 428 & 375 & 395 & 420 & 440 \\
\hline Barley [a] & 162 & 139 & 147 & 155 & 164 \\
\hline Corn [b] & 468 & 400 & 420 & 441 & 466 \\
\hline Others [c] & 28 & 26 & 27 & 28 & 30 \\
\hline $\begin{array}{l}\text { Total } \\
\text { Less [d] }\end{array}$ & $\begin{array}{l}3,899 \\
-390\end{array}$ & $\begin{array}{l}3,320 \\
-332\end{array}$ & $\begin{array}{r}3,499 \\
-350\end{array}$ & $\begin{array}{l}3,694 \\
-369\end{array}$ & $\begin{array}{l}3,900 \\
-390\end{array}$ \\
\hline Total available & -390 & $-30<$ & -300 & & -300 \\
\hline for consumption & 3,509 & 2,988 & 3,149 & 3,325 & 3,510 \\
\hline Population & 14,210 & 16,300 & 16,600 & 16,920 & 17,260 \\
\hline Per capita & & & & & \\
\hline consumption (kg) & 250 & 235 & 237 & 238 & 240 \\
\hline Total requirement & 3,552 & 3,830 & 3,934 & 4,027 & 4,142 \\
\hline Deficit (mainly wheat) & (43) & (842) & $(785)$ & (702) & (632) \\
\hline $\begin{array}{l}\text { Other consumer goods } \\
\text { Sugar (000 tons) }\end{array}$ & & & & & \\
\hline Domestic production & 11 & 2 & 4 & 7 & 10 \\
\hline Requirement & 91 & 130 & 133 & 136 & 138 \\
\hline Deficit & (80) & (128) & (129) & (129) & (128) \\
\hline Vegetable oil (000 tons) & & & & & \\
\hline Domestic production & 10 & 5 & 7 & 8 & 10 \\
\hline Requirement & 30 & 70 & 65 & 58 & 50 \\
\hline Deficit & (20) & (65) & $(58)$ & (50) & (40) \\
\hline Non-Food Requirements & & & & & \\
\hline Cotton textile (000 tons) & & & & & \\
\hline Domestic production & 88 & 60 & 65 & 70 & 80 \\
\hline Requirement & 115 & 144 & 148 & 153 & 155 \\
\hline Deficit & $(27)$ & (84) & (83) & (83) & (75) \\
\hline Petroleum products (000 tons) & & & & & \\
\hline Domestic production & 0 & 0 & 0 & 0 & 0 \\
\hline Requirement & 260 & 400 & 410 & 420 & 430 \\
\hline Deficit & $(260)$ & $(400)$ & $(410)$ & $(420)$ & (430) \\
\hline Agricultural Inputs & & & & & \\
\hline Fertilizer (000 tons) & & & & & \\
\hline Domestic production & 63 & 100 & 107 & 120 & 126 \\
\hline Requirement & 98 & 150 & 160 & 180 & 200 \\
\hline Deficit & (35) & $(50)$ & (53) & $(60)$ & (74) \\
\hline Wheat Seed (000 tons) & & & & & \\
\hline Domestic production & 5 & 5 & 8 & 10 & 12 \\
\hline Requirement & 10 & 15 & 17 & 19 & 20 \\
\hline Deficit & (5) & (10) & (9) & (9) & (8) \\
\hline Cotton Seed (000 tons) & & & & & \\
\hline Domestic production & 1 & & & 1 & 2 \\
\hline Requirement & 6 & 4 & 5 & 5 & 6 \\
\hline Deficit & (5) & (4) & (5) & (4) & (4) \\
\hline
\end{tabular}

[a] $50 \%$ of barley crop is assumed to be available for human consumption.

[b] $60 \%$ of corn crop is assumed to be available for human consumption.

[c] $80 \%$ of other grains are assumed to be for human consumption.

[d] Seed requirement and wastage in storage and transportation are assumed to

claim $10 \%$ of total production.

Source: UNDP Discussion Paper on Afghanistan of April 1988. 
Table A II-25. Estimated Industrial and Mining Production

\begin{tabular}{|c|c|c|c|c|c|c|c|}
\hline Products & Units & $1978 / 79$ & $1982 / 83$ & $1983 / 84$ & $1984 / 85$ & $1985 / 86$ & $1986 / 87$ \\
\hline \multicolumn{8}{|c|}{ Mining and Quarrying } \\
\hline Natural Gas & Million cubic meters & 2,461 & 2,583 & 2,767 & 2,820 & 2,761 & 2,425 \\
\hline Coal & Thousand tons & 218 & 145 & 140 & 148 & 151 & 160 \\
\hline Salt & Thousand tons & 81 & 45 & 52 & 53 & 51 & 37 \\
\hline \multicolumn{8}{|l|}{ Food Processing } \\
\hline Wheat Flour & Thousand tons & 97.0 & 124.0 & 133.0 & 154.0 & 174.0 & 187.0 \\
\hline Bakery Products & Thousand tons & 20.4 & -- & -- & 32.2 & 33.6 & 34.1 \\
\hline Sugar & Thousand tons & 10.8 & 1.1 & 0.3 & 0.7 & 0.2 & 0.2 \\
\hline Vegetable Oils & Thousand tons & 10.3 & 2.8 & 2.5 & 2.7 & 3.6 & 3.4 \\
\hline \multicolumn{8}{|c|}{ Textile \& Leather Goods } \\
\hline Ginned Cotton & Thousand tons & 41.5 & -- & -- & 11.0 & 15.0 & 13.0 \\
\hline Cotton Textiles & Million meters & 88.5 & -- & -- & 45.0 & 55.0 & 58.0 \\
\hline Rayon Textiles & Million meters & 23.0 & 6.7 & -- & 3.7 & 4.6 & 4.3 \\
\hline Woolen Textiles & Million meters & 0.4 & 0.2 & 0.2 & 0.3 & 0.2 & 0.2 \\
\hline Shoes (Leather) & Thousand pairs & 304 & 238 & 228 & 296 & 380 & 604 \\
\hline Chemical Fertilizer & Thousand tons & 105 & 113 & 113 & 120 & 125 & 126 \\
\hline \multicolumn{8}{|l|}{ Other } \\
\hline Cement & Thousand tons & 126 & 75 & 107 & 112 & 127 & 103 \\
\hline Electricity & Million Kwh & 845 & 1,017 & 976 & 1,019 & 1,057 & 1,170 \\
\hline
\end{tabular}

Source: Central Statistical Office; cited from the UNDP Discussion Paper on Afghanistan of April 1988. 
Table A II-26. Estimated Industrial and Mining Production

\begin{tabular}{|c|c|c|c|c|c|c|c|c|c|c|c|}
\hline Products & Units & $1977 / 78$ & $1978 / 79$ & $1979 / 80$ & $1980 / 81$ & $1981 / 82$ & $1982 / 83$ & $1983 / 84$ & $1984 / 85$ & $1985 / 86$ & $1986 / 87$ \\
\hline \multicolumn{12}{|l|}{ Mining and quarrying } \\
\hline Natural gas & mill. cub. M & 2583.6 & 2461.0 & 2327.0 & 2790.3 & 2674.6 & 2583.0 & 2767.0 & 2819.9 & 2761.5 & 2700.0 \\
\hline Coal & 1000 tons & 171.9 & 218.2 & 131.9 & 118.7 & 125.0 & 145.4 & 145.3 & 148.3 & 151.4 & 160.0 \\
\hline Salt & 1000 tons & 77.6 & 81.1 & 67.6 & 37.1 & 30.4 & 45.1 & 51.8 & 53.0 & 51.0 & 60.0 \\
\hline Marble & 1000 cub. $M$ & 6.4 & 3.6 & 13.7 & 9.2 & 5.2 & --- & 32.1 & 22.3 & 37.5 & 34.2 \\
\hline \multicolumn{12}{|l|}{ Food processing } \\
\hline Wheat flour & 1000 tons & 59.9 & 97.0 & 122.6 & 113.1 & 122.9 & 123.9 & 136.4 & 153.5 & 173.9 & 170.0 \\
\hline Bakery products & 1000 tons & 20.7 & 20.4 & 21.9 & 20.0 & 20.1 & 25.4 & 30.0 & 32.2 & 33.4 & 30.0 \\
\hline Sugar & 1000 tons & 11.2 & 9.2 & 8.7 & 2.7 & 1.8 & 1.1 & 0.3 & 0.7 & 0.2 & 1.0 \\
\hline Vegetable oil & 1000 tons & 13.0 & 10.4 & 9.5 & 6.5 & 4.5 & 2.8 & 2.1 & 2.7 & 3.6 & 5.4 \\
\hline \multicolumn{12}{|l|}{ Textile and leather } \\
\hline Ginned cotton & 1000 tons & 42.1 & 45.3 & 29.4 & 23.0 & 14.0 & 12.0 & 8.7 & 11.4 & 15.8 & 21.4 \\
\hline Cotton textiles & mill. sq. M & 77.0 & 77.0 & 64.6 & 43.3 & 37.3 & 38.6 & 37.8 & 45.3 & 55.0 & 60.0 \\
\hline Rayon textiles & mill. sq. M & 29.7 & 23.1 & 21.3 & 14.8 & 6.9 & 6.7 & 6.7 & 3.7 & 4.6 & 5.5 \\
\hline Woolen textiles & mill. sq. M & 0.2 & 0.3 & 0.4 & 0.4 & 0.1 & 0.2 & 0.2 & 0.3 & 0.3 & 0.3 \\
\hline Shoes & 1000 pairs & 298.6 & 304.9 & 309.0 & 264.5 & 238.1 & 228.5 & 296.7 & 343.6 & 380.1 & 400.0 \\
\hline \multicolumn{12}{|l|}{ Chemical products } \\
\hline Chemical fertilizers & 1000 tons & 99.6 & 105.7 & 106.2 & 106.3 & 107.8 & 112.8 & 125.3 & 120.5 & 125.5 & 110.0 \\
\hline \multicolumn{12}{|l|}{ Other } \\
\hline Cement & 1000 tons & 150.1 & 125.6 & 99.3 & 87.2 & 77.1 & 107.8 & 130.5 & 111.6 & 127.6 & 136.0 \\
\hline Electricity & mill. kwatt hrs & 763.8 & 857.4 & 907.8 & 965.2 & 1017.0 & 976.0 & 1039.3 & 1027.4 & 1057.5 & 1173.0 \\
\hline
\end{tabular}

Source: Afghanistan: Recent Economic Developments, IMF, 1983-1986. From the Government of Afghanistan, Central Statistical Office and Ministry of Mines and Industries. 
Table A II-27. Pre-war Coal Production (cubic tons)

\begin{tabular}{|c|c|c|c|c|c|c|c|c|c|}
\hline $1967 / 68$ & $1968 / 69$ & $1969 / 70$ & $1970 / 71$ & $1971 / 72$ & $1972 / 73$ & $1973 / 74$ & $1974 / 75$ & $1975 / 76$ & $1976 / 77$ \\
\hline
\end{tabular}

Source: "The USSR and Afghanistan Mineral Resources," by John F. Shroder, Jr., in International Minerals: A National Perspective, Allen F. Agnew, Ed., 1983, p. 133; cited from the Orkand Report. 
Table A II-28. Natural Gas Production

(Millions of cubic meters)

\begin{tabular}{|c|c|c|c|c|c|c|c|c|c|}
\hline $1967 / 68$ & $1968 / 69$ & $1969 / 70$ & $1970 / 71$ & $1971 / 72$ & $1972 / 73$ & $1973 / 74$ & $1974 / 75$ & $1975 / 76$ & $1976 / 77$ \\
\hline 595 & 1681 & -- & -- & 2528 & 2871 & 2767 & 2918 & 2995 & 2641 \\
\hline
\end{tabular}

Source: "The USSR and Afghanistan Mineral Resources," by John F. Shroder, Jr., in

International Minerals: A National Perspective, Allen F. Agnew, Ed., 1983, p. 133; cited

from the Orkand Report. 


\section{PUBLIC FINANCE}

1. IMF Summary Statement of Government Finances

2. UNDP Summary of Budgetary Accounts

3. IBRD Summary Statement of Central Government Finances.

4. IMF Summary of Central Government Budgetary Revenue

5. UNDP Summary of Central Government Revenue

6. IBRD Summary of Central Government Revenue

7. IMF Summary of Central Government Ordinary Budgetary Expenditure

8. IBRD Summary of Central Government Ordinary Expenditure

9. IBRD Summary of Gross and Adjusted Central Government Revenues

0. RETURN TO MAIN MENU 
Table A III-2. UNDP Summary of Budgetary Accounts

(millions of afghanis, unless otherwise indicated)

\begin{tabular}{|c|c|c|c|c|}
\hline & $1978 / 79$ & $1984 / 85$ & $1985 / 86$ & $\begin{array}{l}1986 / 87 \\
\text { (prelim.) }\end{array}$ \\
\hline A. Total Revenue & 16,455 & 36,600 & 41,940 & 45,352 \\
\hline Tax Revenue & 9,448 & 16,427 & 17,489 & 18,911 \\
\hline Non-tax Revenue & 7,007 & 20,173 & 24,451 & 26,441 \\
\hline \multirow{4}{*}{$\begin{array}{l}\text { B. Recurrent Expenditure } \\
\text { Government Departments } \\
\text { Subsidies } \\
\text { Amortization \& Interest } \\
\text { (gross) }\end{array}$} & 12,384 & 44,529 & 52,425 & 63,672 \\
\hline & 8,866 & 35,361 & 42,925 & 54,086 \\
\hline & 1,024 & 3,868 & 4,580 & 4,800 \\
\hline & 2,494 & 5,300 & 4,920 & 4,786 \\
\hline C. Current Balance $(A-B)$ & 4,071 & $(7,929)$ & $(10,485)$ & $(18,320)$ \\
\hline D. Development Expenditure & 14,013 & 13,200 & 16,000 & 18,500 \\
\hline Budgetary & 6,844 & 8,174 & 8,967 & 10,137 \\
\hline Project Aid (gross) & 7,169 & 5,026 & 7,033 & 8,363 \\
\hline E. Overall Deficit (C-D) & $(9,942)$ & $(21,129)$ & $(26,485)$ & $(36,820)$ \\
\hline $\begin{array}{l}\text { Financing } \\
\text { Foreign }\end{array}$ & 9,942 & 21,129 & 26,485 & 36,820 \\
\hline Project Aid (gross) & 7,168 & 5,026 & 7,033 & 8,363 \\
\hline Commodity Aid & 1,745 & 5,513 & 6,812 & 11,400 \\
\hline Domestic & & & & \\
\hline $\begin{array}{l}\text { Central Bank } \\
\text { Other }\end{array}$ & 1,029 & 10,590 & 12,640 & 17,057 \\
\hline \multicolumn{5}{|l|}{ Memorandum Items } \\
\hline Total Expenditure & 26,397 & 57,729 & 68,425 & 82,172 \\
\hline $\begin{array}{l}\text { Consumer Price Index } \\
(1978 / 79=100)\end{array}$ & 100 & 241 & 260 & 281 \\
\hline $\begin{array}{l}\text { Exchange Rate (Official) } \\
\text { afghanis to U.S. dollar }\end{array}$ & 45 & 50 & 50 & 50 \\
\hline
\end{tabular}


(millions of afghanis)

\begin{tabular}{|c|c|c|c|c|c|c|}
\hline & $1972 / 73$ & $1973 / 74$ & $1974 / 75$ & $1975 / 76$ & $\begin{array}{l}\text { Revised } \\
\text { Estimates } \\
1976 / 77 \text { [a] }\end{array}$ & $\begin{array}{l}\text { Budget } \\
1977 / 78\end{array}$ \\
\hline A. Domestic Revenue & 5,750 & 6,637 & 9,669 & 11,298 & 12,810 & 14,082 \\
\hline Tax revenue & ---- & $---\overline{4,056}$ & $----\overline{6,585}$ & 6,709 & $---\frac{--}{7,934}$ & 8,629 \\
\hline Non-tax revenue & 2,492 & 2,506 & 2,940 & 4,460 & 4,752 & 5,265 \\
\hline Capital revenue & 62 & 75 & 144 & 129 & 124 & 188 \\
\hline B. Ordinary Expenditure & 4,923 & 5,419 & 5,663 & 8,354 & 9,587 & 9,790 \\
\hline Budgetary [b] & 4,744 & 5,220 & 5,443 & 8,070 & 9,353 & $\overline{9,790}$ \\
\hline Extrabudgetary (interest) [c] & 179 & 199 & 220 & 284 & 234 & 0 \\
\hline C. Development Expenditure & 4,326 & 3,672 & 4,648 & 6,065 & 11,500 & 20,233 \\
\hline Budgetary & 2,387 & $1,-\overline{986}$ & 2,373 & 2.780 & 4,300 & $6,-\overline{650}$ \\
\hline Project aid [d] & 1,939 & 1,686 & 2,275 & 3,285 & 4,000 & 13,583 \\
\hline Extrabudgetary & 0 & 0 & 0 & 0 & 3,200 & 0 \\
\hline D. Total Expenditure & 9,249 & 9,091 & 10,311 & 14,419 & 21,087 & 30,023 \\
\hline Ordinary Surplus $(A-B)$ & 827 & 1,218 & 4,006 & 2,944 & 3,223 & 4,292 \\
\hline Overall deficit $(A-D)$ & $(3,499)$ & $(2,454)$ & (642) & $(3,121)$ & $(8,277)$ & $(15,941)$ \\
\hline Financing & 3,499 & 2,454 & 642 & 3,121 & 8,277 & 15,941 \\
\hline--------- & ---- & --- & ---- & -- & ---- & --- \\
\hline Foreign Aid (net) [e] & 2,634 & 1,377 & 1,645 & 3,709 & 4,423 & 13,641 \\
\hline Project aid & 1,939 & 1,686 & 2,275 & 3,285 & 4,000 & 13,583 \\
\hline Commodity aid [f] & 1,250 & 622 & 683 & 1,359 & 1,400 & 1,495 \\
\hline Amortization & $(555)$ & (931) & $(1,313)$ & (935) & (977) & $(1,437)$ \\
\hline $\begin{array}{l}\text { Central Bank Borrowing } \\
\text { Adjustment to a cash basis \& }\end{array}$ & 39 & 1,046 & (598) & $(817)$ & 3,854 & 2,300 \\
\hline Statistical Discrepancy & 826 & 31 & (405) & 229 & 0 & 0 \\
\hline
\end{tabular}

[a] The revised estimates for $1976 / 77$ are based on provisional actuals for ten months.

[b] The budgetary data for ordinary expenditure have been adjusted to exclude foreign debt

amortization, which is treated as a negative financing item.

[c] Represents interest payments on government domestic loans at Da Afghanistan Bank.

[d] Includes foreign grants. In addition to cash grants, commodity aid includes the proceeds in

local currency of sales of commodity loans and grants.

[e] Includes cash grants.

complementary period after the end of the fiscal year, and changes in cash balances of loca

treasuries held outside the banking system.

Sources: Data provided by the GOA Ministry of Finance, Da Afghanistan Bank, and the Ministry of

Planning: cited from "Afghanistan: The Journey to Economic Development", Vol. II, March 1978. 
Table A III -4. IMF Summary of Central Govemment Budgetary Revenue

(millions of afghenis)

\begin{tabular}{|c|c|c|c|c|c|c|c|c|c|}
\hline & $1978 / 79$ & $1979 / 80$ & 1980/81 & $1981 / 82$ & $1982 / 83$ & $1983 / 84$ & $1984 / 85$ & $1985 / 88^{B}$ & $\begin{array}{l}\text { udgd } \\
1986 / 87\end{array}$ \\
\hline Tax revenue & 8,852 & 7,146 & 7,352 & 8,844 & 10,789 & 13,169 & 16,232 & 18,142 & 21,702 \\
\hline Taxes on income and profits & 1,419 & 1,446 & 999 & 1,085 & 1,282 & 1,525 & 2,033 & 2,619 & 3,955 \\
\hline Corporate income taxes & 597 & 625 & 334 & 336 & 386 & 429 & 546 & 705 & 740 \\
\hline Individual income taxes & 822 & 821 & 665 & 749 & 896 & 1,096 & 1,487 & 1,914 & 3,215 \\
\hline Taxes on property & 280 & 67 & 35 & 16 & 78 & 79 & 129 & 122 & 135 \\
\hline Taxes on goods and services & 789 & 658 & 640 & 538 & 601 & 666 & 728 & 900 & 1,095 \\
\hline Sales tax & 154 & 160 & 170 & 104 & 80 & 86 & 82 & 100 & 130 \\
\hline Licenses [a] & 635 & 498 & 470 & 434 & 521 & 580 & 644 & 800 & 965 \\
\hline \multicolumn{10}{|l|}{ Taxes on intemational trade } \\
\hline 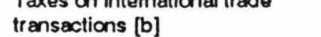 & 4,819 & 3.257 & 3,855 & 4,348 & 5,513 & 5,881 & 10.478 & 11,101 & 11,965 \\
\hline $\begin{array}{l}\text { Transactions } \\
\text { Import duties }\end{array}$ & $\begin{array}{l}4,619 \\
4,473\end{array}$ & 2,912 & $\begin{array}{l}3,050 \\
3,558\end{array}$ & $\begin{array}{l}4,011 \\
4,011\end{array}$ & 5,133 & $\begin{array}{l}5,501 \\
5,532\end{array}$ & $\begin{array}{l}0,476 \\
10,158\end{array}$ & 10,692 & 11,550 \\
\hline $\begin{array}{l}\text { Import anties } \\
\text { Export duties }\end{array}$ & $\begin{array}{l}4,473 \\
346\end{array}$ & $\begin{array}{r}2,912 \\
345\end{array}$ & 297 & $\begin{array}{ll}4,0117 \\
337\end{array}$ & 380 & $\begin{array}{l}3,32 \\
349\end{array}$ & 10,320 & $\begin{array}{r}10,092 \\
409\end{array}$ & 405 \\
\hline Other taxes [c] & 1,545 & 1,718 & 1,823 & 2,857 & 3,315 & 5,018 & 2,806 & 3,400 & 4,502 \\
\hline Nontax revenue & 6.986 & 8.591 & 18.787 & 20,967 & 21,766 & 20,951 & 26.865 & 33,202 & 36.919 \\
\hline Sales of natural gas & 2,637 & 3,873 & 11,509 & 13,556 & 14,792 & 15,137 & 13,783 & 17,326 & 15,468 \\
\hline Income from public enterprises & 1,670 & 2,164 & 3,005 & 5,390 & 4,951 & 3,680 & 4,075 & 6,220 & 9,387 \\
\hline State enterprises & -- & - & 1.920 & 2,426 & 1.214 & 1,269 & 1,761 & 3,056 & 5,000 \\
\hline Mixed enterprises and banks & -- & -- & 1.076 & 2,964 & 3,737 & 2,411 & 2,314 & 3,164 & 4,387 \\
\hline Administrative fees and charges & 596 & 107 & 2,574 & 196 & 958 & 1,103 & 696 & 1.161 & 1,750 \\
\hline Other [d] & 2,083 & 2,447 & 1,699 & 1,825 & 1,065 & 1,031 & 8,311 & 8,585 & 10,314 \\
\hline Total revenue & 15,838 & 15,737 & 26,139 & 29,811 & 32,555 & 34,120 & 43,097 & 51,434 & 58,621 \\
\hline
\end{tabular}

[a] Includes motor vehicle registration lees.
[b] Includes revenue from fixed Import and export fees.

[c] Includes revenue from

(d) Excludes unrealized silver revaluation proflts of Af 1394 million in 1982/83 and an IMF drawing of AI 796

million in 1983/84.

Source: GOA Ministry of Finance, and Research Department, Da Afghanistan Bank; cited from Afghanistan: Recent Economic

Developments, IMF, 1983-86.

Table A III -4. IMF Summary of Central Govemment Budgetary Revenue

(millions of afghanis)

\begin{tabular}{|c|c|c|c|c|c|c|c|c|c|c|}
\hline & Actual & $\begin{array}{r}1978 / 79 \\
\text { corr. fact. }\end{array}$ & adj. fig. & Actual & $\begin{array}{r}1979 / 80 \\
\text { corr. fact. }\end{array}$ & adj. fig. & Actual & $\begin{array}{r}1980 / 81 \\
\text { corr. fact. }\end{array}$ & adj. fig. & Actua \\
\hline Tax revenue & 8,852 & & 8,852 & 7.146 & & 7,146 & 7,352 & & 7,352 & $8,844>>>>3$ \\
\hline Taxes on income and profis & 1,419 & & 1,419 & 1,446 & & 1,446 & 999 & & 999 & 1,085 \\
\hline Corporate income taxes & 597 & 1.00 & 597 & 625 & 1.00 & 625 & 334 & 1.00 & 334 & 336 \\
\hline Individual Income taxes & 822 & 1.00 & 822 & 821 & 1.00 & 821 & 665 & 1.00 & 665 & 749 \\
\hline Taxes on property & 280 & 1.00 & 280 & 67 & 1.00 & 67 & 35 & 1.00 & 35 & 16 \\
\hline Taxes on goods and services & 789 & & 789 & 658 & & 658 & 640 & & 640 & 538 \\
\hline Sales tax & 154 & 1.00 & 154 & 160 & 1.00 & 160 & 170 & 1.00 & 170 & 104 \\
\hline \multirow{2}{*}{\multicolumn{11}{|c|}{ Taxes on intemational trade }} \\
\hline & & & & & & & & & & \\
\hline transactions [b] & 4,819 & & 4.819 & 3.257 & & 3,257 & 3,855 & & 3,855 & 4,348 \\
\hline Import duties & 4,473 & 1.00 & 4,473 & 2,912 & 1.00 & 2,912 & 3,558 & 1.00 & 3558 & 4,011 \\
\hline Export duties & 346 & 1.00 & 346 & 345 & 1.00 & 345 & 297 & 1.00 & 297 & 337 \\
\hline Other taxes $[c]$ & 1,545 & 1.00 & 1,545 & 1,718 & 1.00 & 1.718 & 1,823 & 1.00 & 1823 & 2,857 \\
\hline Nontax revenue & 6,986 & & 6,986 & 8,591 & & 8,591 & 18.787 & & 18,787 & 20,967 \\
\hline Sales of natural g & 2,637 & 1.00 & 2,637 & 3,873 & 1.00 & 3,873 & 11,509 & 1.00 & 11509 & 13,556 \\
\hline Income from public enterprises & 1,670 & & 1,670 & 2,164 & & 2,164 & 3,005 & & 3,005 & 5,390 \\
\hline State enterprises & -- & 1.00 & 0 & -- & 1.00 & 0 & 1,920 & 1.00 & 1929 & 2,426 \\
\hline Mixed enterprises and banks & -- & 1.00 & 0 & -- & 1.00 & 0 & 1,076 & 1.00 & 1076 & 2,964 \\
\hline Administrattve fees and charges & 596 & 1.00 & 596 & 107 & 1.00 & 107 & 2.574 & 1.00 & 2574 & 196 \\
\hline Other [d] & 2,083 & 1.00 & 2,083 & 2.447 & 1.00 & 2,447 & 1,699 & 1.00 & 1690 & 1,825 \\
\hline Total revenue & 15,838 & & 15,838 & 15,737 & & 15,737 & 28,139 & & 26,139 & 29,811 \\
\hline
\end{tabular}

[a] Includes motor vehicle registration lees.

[c] Includus stamp taxes

million in 1983/84. 
Table A III-5. UNDP Summary of Central Government Revenue (millions of afghanis)

\begin{tabular}{|c|c|c|c|c|}
\hline Current Revenue & $1978 / 79$ & $1984 / 85$ & $1985 / 86$ & $1986 / 87$ \\
\hline Tax Revenue & 9,449 & 16,427 & 17,489 & 18,911 \\
\hline Direct Taxes & 2,535 & 4,627 & 5,393 & 5,850 \\
\hline Corporate Income Taxes & 1,393 & 2,937 & 3,322 & 3,603 \\
\hline Individual Income Taxes & 823 & 1,587 & 1,917 & 2,083 \\
\hline $\begin{array}{r}\text { Taxes on Property } \\
\text { - Land Tax }\end{array}$ & 280 & 30 & 23 & 23 \\
\hline - Livestock Tax & - - & 37 & 87 & 94 \\
\hline Other Direct Income Taxes & 39 & 36 & 44 & 47 \\
\hline Indirect Taxes & 6,914 & 11,800 & 12,096 & 13,061 \\
\hline Taxes on Foreign Trade & 5,822 & 10,901 & 10,873 & 11,741 \\
\hline -Import Duties & 5,466 & 10,563 & 10,421 & 11,245 \\
\hline - Export Duties & 356 & 338 & 452 & 496 \\
\hline Other Indirect Taxes & 1,092 & 899 & 1,223 & 1,320 \\
\hline $\begin{array}{l}\text { Non-tax Revenue } \\
\text { Income from Sale of State }\end{array}$ & 7,009 & 20,171 & 24,453 & 26,441 \\
\hline Property, Goods \& Services & 3,480 & 14,716 & 18,171 & 19,637 \\
\hline - Sales of Natural Gas & 2,637 & 13,783 & 15,450 & 13,000 \\
\hline -Others & 843 & 933 & 2,721 & 6,637 \\
\hline $\begin{array}{l}\text { Licenses, Fees \& Rates } \\
\text { Income from Use of State }\end{array}$ & 635 & 644 & 597 & 635 \\
\hline Property & 478 & 1,660 & 1,466 & 1,587 \\
\hline Public Enterprises & 1,192 & 1,761 & 2,999 & 3,265 \\
\hline Miscellaneous Revenue & 648 & 1,039 & 1,027 & 1,088 \\
\hline -Pension Funds & -- & 694 & 543 & 576 \\
\hline -Other & 648 & 345 & 484 & 512 \\
\hline Non-Revenue Income & 576 & 351 & 193 & 229 \\
\hline Total Revenue & 16,458 & 36,598 & 41,942 & 45,352 \\
\hline \multicolumn{5}{|l|}{ Memorandum Items: } \\
\hline Commodity Assistance & 1745 & 5513 & 6812 & 11400 \\
\hline $\begin{aligned} & \text { Consumer Price Index } \\
&(1978 / 79=100)\end{aligned}$ & 100 & 241.3 & 260.1 & 280.9 \\
\hline
\end{tabular}

Source: Central Statistical Office; cited from UNDP Discussion Paper on Afghanistan of April 1988. 


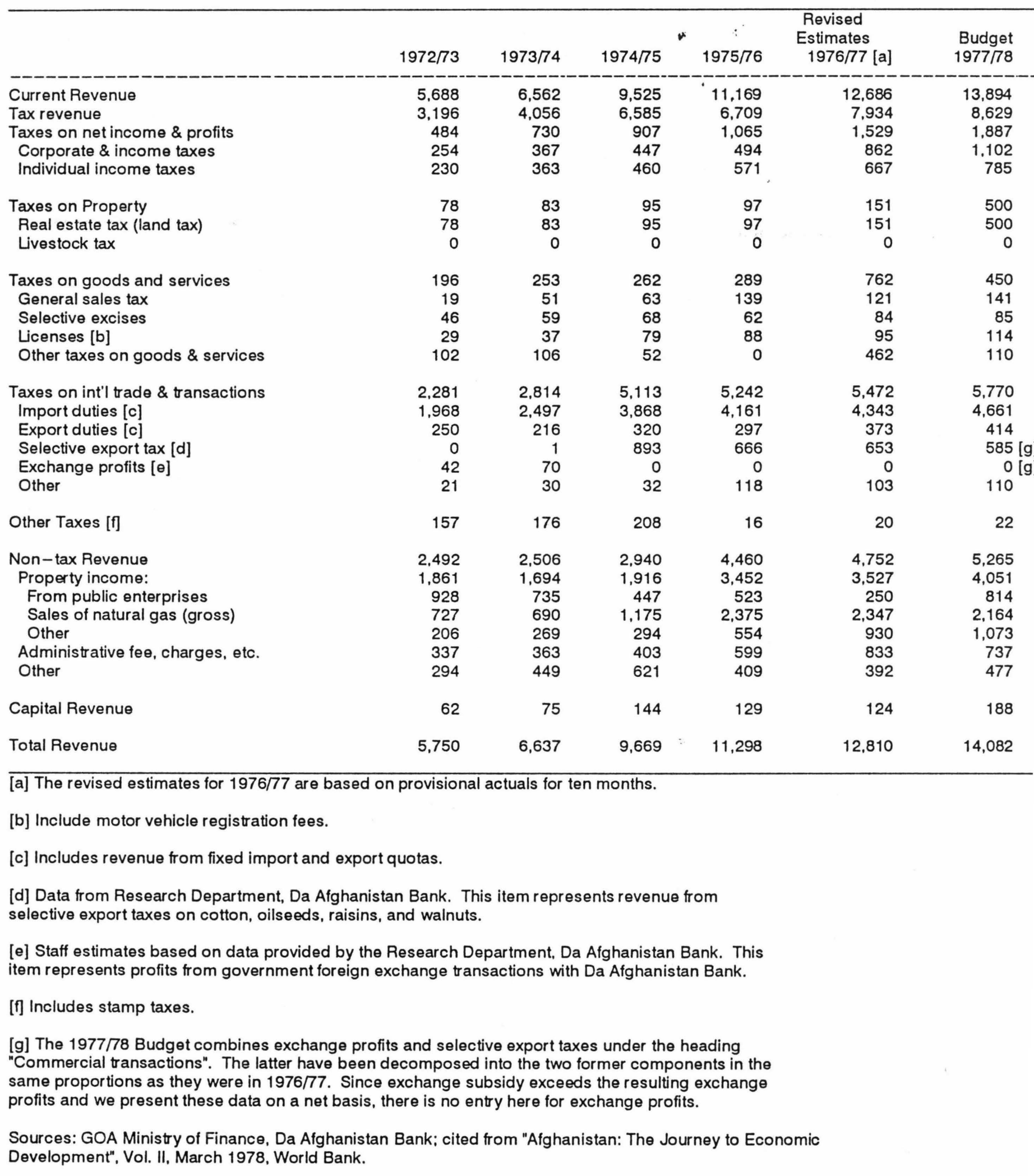




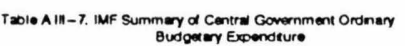

(mitions od atoneres)

\begin{tabular}{|c|c|c|c|c|c|c|c|c|c|}
\hline & 19rers & 1979700 & 1960/191 & $1901 / 82$ & 19060303 & 1900094 & 190005 & $1906 / 100^{8}$ & 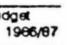 \\
\hline 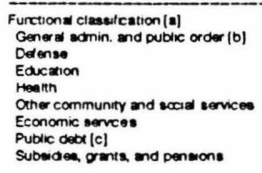 & 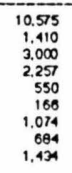 & 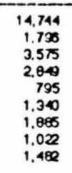 & 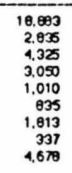 & 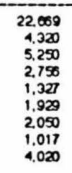 & 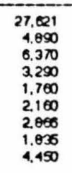 & 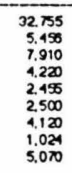 & 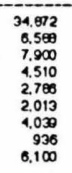 & 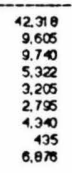 & 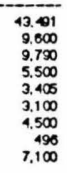 \\
\hline 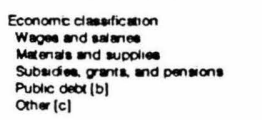 & $\begin{array}{l}10.55 \\
4.25 \\
2.99 \\
1.49 \\
1.04 \\
1.328\end{array}$ & 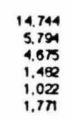 & 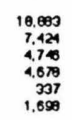 & $\begin{array}{l}220000 \\
11.000 \\
5,30 \\
4.020 \\
1.017 \\
1.200\end{array}$ & 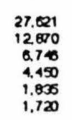 & $\begin{array}{l}32.55 \\
13.950 \\
10.755 \\
5.070 \\
1.024 \\
1.98\end{array}$ & $\begin{array}{l}34.822 \\
14.900 \\
11.077 \\
6.1100 \\
1930 \\
1.989\end{array}$ & $\begin{array}{l}22318 \\
20.05 \\
12500 \\
6.978 \\
305 \\
2000\end{array}$ & $\begin{array}{l}43.41 \\
22000 \\
12700 \\
7.100 \\
490 \\
2.405\end{array}$ \\
\hline
\end{tabular}

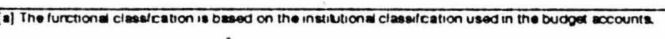

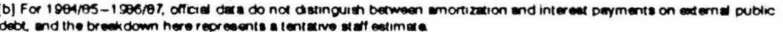

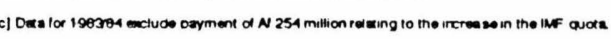

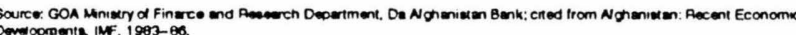

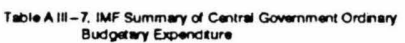

untions o d a tonarese)

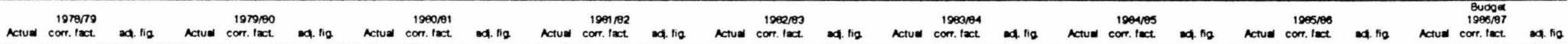

\begin{tabular}{|c|c|c|c|c|c|c|c|c|c|c|c|c|c|c|c|c|c|c|c|c|c|c|c|c|c|c|c|}
\hline 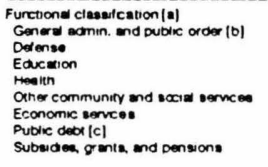 & 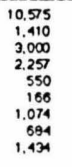 & $\begin{array}{l}1.00 \\
1.00 \\
1.00 \\
1.00 \\
1.00 \\
1.00 \\
1.00 \\
1.00\end{array}$ & $\begin{array}{l}10.55 \\
1.140 \\
1.100 \\
2.25 \\
550 \\
1.68 \\
1.674 \\
1.694 \\
1.434\end{array}$ & 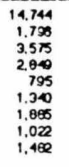 & $\begin{array}{l}100 \\
1.00 \\
100 \\
100 \\
1.00 \\
100 \\
1.00 \\
1.00\end{array}$ & 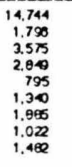 & 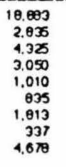 & $\begin{array}{l}1.00 \\
1.00 \\
100 \\
100 \\
1.00 \\
1.00 \\
1.00 \\
1.00\end{array}$ & 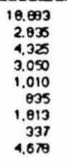 & 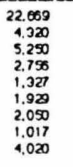 & $\begin{array}{l}1.00 \\
1.00 \\
1.00 \\
1.00 \\
1.00 \\
1.00 \\
1.00\end{array}$ & 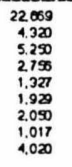 & 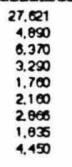 & $\begin{array}{l}1.00 \\
100 \\
100 \\
100 \\
100 \\
1.00 \\
1.00 \\
1.00\end{array}$ & 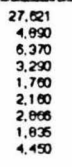 & 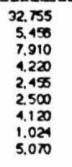 & $\begin{array}{l}1.00 \\
1.00 \\
1.00 \\
100 \\
1.00 \\
1000 \\
1.00\end{array}$ & 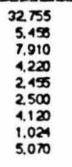 & 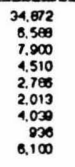 & $\begin{array}{l}1.00 \\
1.00 \\
100 \\
1.00 \\
1.00 \\
1.00 \\
1.00\end{array}$ & 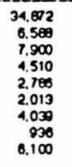 & 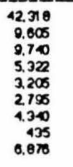 & $\begin{array}{l}1.00 \\
1.00 \\
1.00 \\
1.00 \\
1.00 \\
1.00 \\
1.00\end{array}$ & 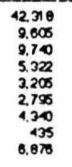 & 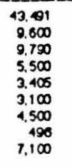 & $\begin{array}{l}1.00 \\
1.00 \\
1.00 \\
1.00 \\
1.00 \\
1.00 \\
1.00\end{array}$ & 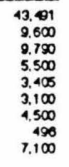 \\
\hline 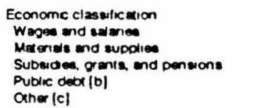 & 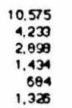 & $\begin{array}{l}1.00 \\
1.00 \\
1.00 \\
1.00 \\
1.00\end{array}$ & 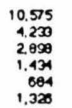 & 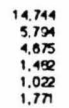 & $\begin{array}{l}1.00 \\
1.00 \\
1.00 \\
1.00 \\
1.00\end{array}$ & 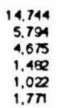 & 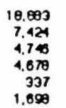 & $\begin{array}{l}1.00 \\
1.00 \\
1.00 \\
1.00 \\
1.00\end{array}$ & 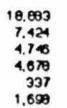 & 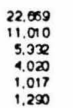 & $\begin{array}{l}1.000 \\
1.00 \\
1.00 \\
1.00 \\
1.00\end{array}$ & 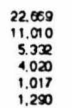 & 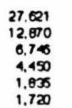 & $\begin{array}{l}1.00 \\
100 \\
100 \\
1.00 \\
1.00\end{array}$ & 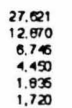 & 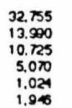 & $\begin{array}{l}1.00 \\
1000 \\
1.00 \\
1.00 \\
1.00\end{array}$ & 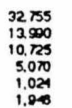 & 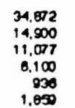 & $\begin{array}{l}1.00 \\
1.00 \\
1.00 \\
1.00\end{array}$ & 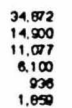 & 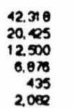 & $\begin{array}{l}1.00 \\
1.00 \\
1.00 \\
1.00 \\
1.00\end{array}$ & $\begin{array}{l}42.38 \\
20.95 \\
12500 \\
6.978 \\
435 \\
2.068\end{array}$ & $\begin{array}{l}43.41 \\
20.700 \\
12700 \\
1.100 \\
490 \\
2405\end{array}$ & $\begin{array}{l}1.00 \\
1.00 \\
1.00 \\
1.00 \\
1.00\end{array}$ & 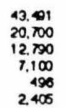 \\
\hline
\end{tabular}

The

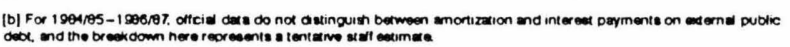

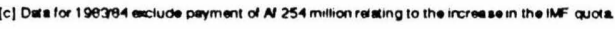

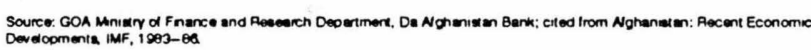


Table A III-8. World Bank Summary of Central

Government Ordinary Expenditure

(millions of afghanis)

\begin{tabular}{|c|c|c|c|c|c|c|}
\hline & $1972 / 73$ & $1973 / 74$ & $1974 / 75$ & $1975 / 76$ & $\begin{array}{l}\text { Revised } \\
\text { Estimates } \\
1976 / 77 \text { [a] }\end{array}$ & $\begin{array}{r}\text { Budget } \\
1977 / 78\end{array}$ \\
\hline \multicolumn{7}{|l|}{ - } \\
\hline \multicolumn{7}{|l|}{ Functional Classification [b] } \\
\hline Ordinary expenditure [c] & 4,923 & 5,419 & 5,663 & 8,354 & 9,587 & 9,790 \\
\hline General admin. \& public order & 791 & 880 & 887 & 1,100 & 1,274 & 1,486 \\
\hline Defense & 1,460 & 1,545 & 1,608 & 2,114 & 2,184 & 2,731 \\
\hline Education & 968 & 1,083 & 1,120 & 1,243 & 1,550 & 1,850 \\
\hline Health & 186 & 192 & 204 & 268 & 342 & 379 \\
\hline Other community \& social services & 76 & 137 & 105 & 58 & 61 & 207 \\
\hline Economic services & 422 & 449 & 559 & 837 & 1,022 & 690 \\
\hline Public debt (interest) & 659 & 679 & 750 & 548 & 614 & 690 \\
\hline Foreign [c] & 480 & 480 & 530 & 264 & 380 & 690 \\
\hline Domestic [d] & 179 & 199 & 220 & 284 & $234[e]$ & 0 \\
\hline Subsidies, grants \& pensions & 361 & 454 & 430 & 2,186 & 2,540 & 1,757 \\
\hline Exchange subsidy & & & & 328 & 367 & 397 \\
\hline \multicolumn{7}{|l|}{ Object Classification } \\
\hline UDject Classilication & & & .. & & & \\
\hline Ordinary expenditure & 4,923 & 5,419 & 5,663 & 8,354 & 9,587 & 9,790 \\
\hline Wages and salaries & 2,221 & 2,395 & 2,396 & 2,828 & 3,265 & 3,786 \\
\hline Materials and supplies & 1,160 & 1,269 & 1,417 & 2,646 & 2,891 & 3,540 \\
\hline Subsidies, grants \& pensions & 505 & 637 & 699 & 2,257 & 2,768 & 1,757 \\
\hline Public debt (interest) & 659 & 679 & 750 & 548 & 614 & 690 \\
\hline Foreign [c] & 480 & 480 & 530 & 264 & 380 & 690 \\
\hline Domestic [d] & 179 & 199 & 220 & 284 & 234 & 0 \\
\hline Other & 378 & 439 & 401 & 75 & 49 & 17 \\
\hline
\end{tabular}

[a] The revised estimates for $1976 / 77$ are based on provisional actuals for ten months.

[b] The functional classification is based on the institutional classification used in the budget accounts.

[c] The budgetary item, "foreign debt service", in ordinary expenditure has been adjusted to exclude foreign debt amortization, which is treated as a negative financing item.

[d] Represents interest payments on government domestic loans at Da Afghanistan Bank.

[e] World Bank Staff estimates.

Sources: GOA Ministry of Finance, Da Afghanistan Bank; cited from "Afghanistan: The Journey to Economic 
Table A III-9. World Bank Summary of Gross and Adjusted Central Government Revenues 1975/76 (millions of afghanis)

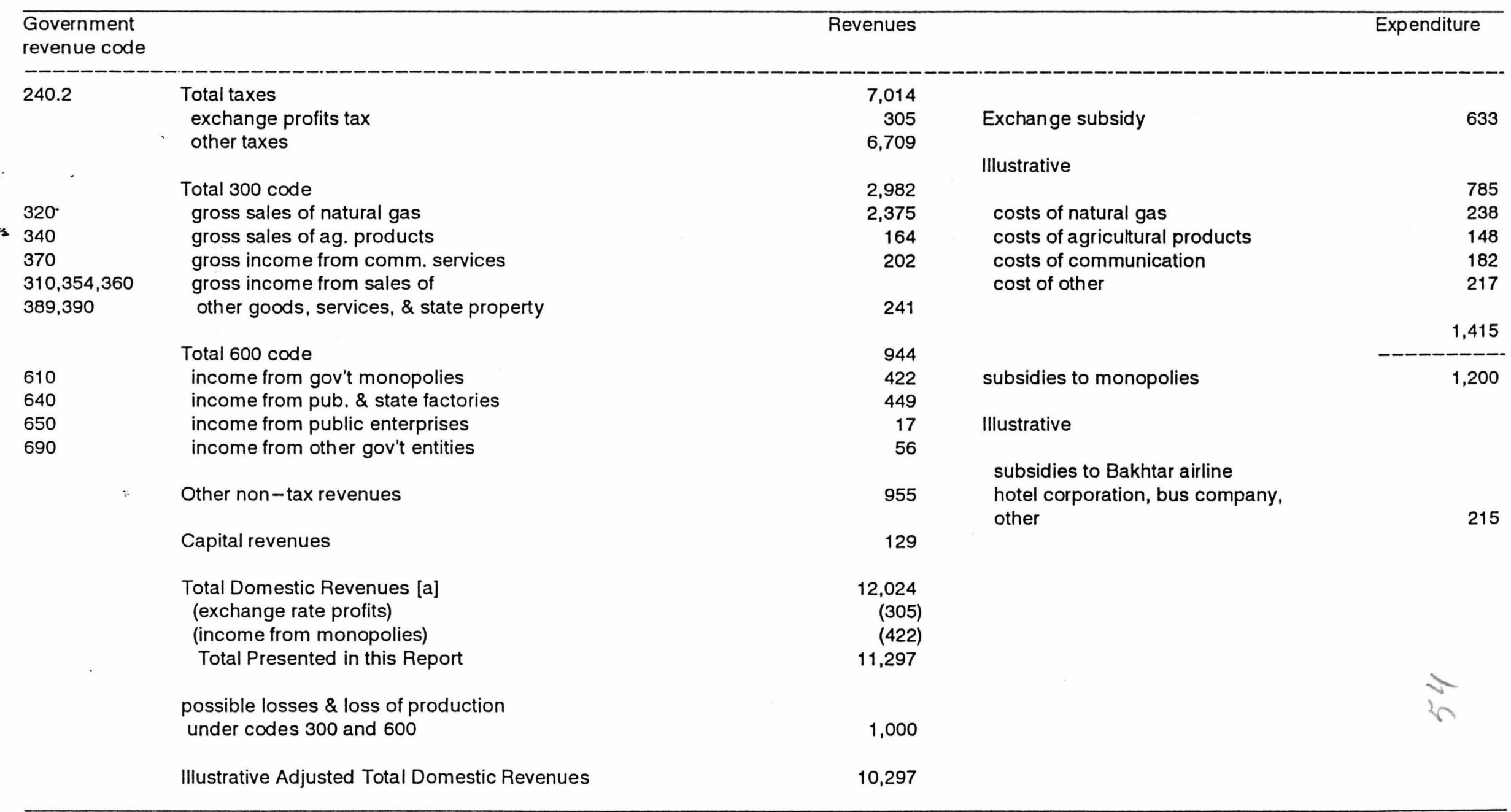

[a] Central government only.

Source: "Afghanistan: The Journey to Economic Development", Vol. II, March 1978, World Bank. 
Table A IV-8. Survey of Average Wheat

Prices in Eastern Provinces

(afghanis per seer)

\begin{tabular}{lrrrr}
\hline & Apr/May & Apr/May & Winter & Apr/May \\
Province & 1985 & 1986 & $1986 / 87$ & 1987 \\
\hdashline Kabul & 125 & 170 & 225 & 260 \\
Kabul City & 165 & 205 & 250 & 270 \\
Kapisa/Parvan & 100 & 150 & -- & 260 \\
Vardak & 145 & 185 & 280 & 265 \\
Lowgar & 120 & 165 & 375 & 235 \\
Ghazni & 140 & 190 & 305 & 235 \\
Paktia & 140 & 200 & 400 & 260 \\
Nangarhar & 100 & 150 & -- & 220 \\
Laghman & 110 & 160 & -- & 250 \\
Kanorha & -- & -- & -- & 300 \\
Bamian & 150 & 180 & 320 & \\
\hline
\end{tabular}

Note: One seer is equal to approximately seven kilograms.

Source: Confidential Crossborder Survey, 1987; cited from the Orkand Report. 
Table A IV -7. Survey of Average Wheat

Prices in Northern Provinces

(afghanis per seer)

\begin{tabular}{lrrrr}
\hline & Apr/May & Apr/May & Winter & Apr/May \\
Province & 1985 & 1986 & $1986 / 87$ & 1987 \\
\hline Konduz & 115 & 150 & 265 & 180 \\
Takhar & 115 & 150 & 285 & 200 \\
Baghlan & 130 & 180 & 345 & 235 \\
Balkh & 115 & 180 & 325 & 265 \\
Badakhshar & 170 & 170 & 300 & 270 \\
Samangan & 125 & 205 & 450 & 355 \\
Faryab & 175 & 325 & 450 & 395 \\
Badghisat & 250 & 335 & 395 & 410 \\
Jowzjan & 185 & 290 & 450 & 410 \\
\hline
\end{tabular}

Note: One seer is equal to approximately seven kilograms.

Source: A.I.D. survey, 1987, untitled, unpublished; cited from the Orkand Report. 
Table A IV -6. Major Consumer Goods Price Indices $(1968 / 69=100)$

\begin{tabular}{|c|c|c|c|c|c|c|c|c|c|}
\hline & $1968 / 69$ & $1969 / 70$ & $1970 / 71$ & $1971 / 72$ & $1972 / 73$ & $1973 / 74$ & $1974 / 75$ & \multicolumn{2}{|c|}{ 1975/76 Weights } \\
\hline \multicolumn{10}{|l|}{ Food } \\
\hline Cereals & 100 & 95 & 135 & 161 & 124 & 95 & 115 & 127 & 0.4167 \\
\hline Meat & 100 & 106 & 112 & 108 & 124 & 147 & 178 & 185 & 0.0767 \\
\hline Fruit & 100 & 115 & 134 & 139 & 121 & 161 & 171 & 150 & 0.0760 \\
\hline Vegetables & 100 & 95 & 120 & 115 & 107 & 144 & 134 & 147 & 0.0409 \\
\hline Other food & 100 & 97 & 100 & 110 & 140 & 139 & 143 & 174 & 0.0366 \\
\hline Clothing & 100 & 117 & 119 & 119 & 117 & 121 & 118 & 122 & 0.0779 \\
\hline Rent & 100 & 100 & 100 & 100 & 100 & 100 & 100 & 100 & 0.0196 \\
\hline \multicolumn{10}{|l|}{ Fuel \& Electricity } \\
\hline Electricity & 100 & 108 & 116 & 114 & 108 & 112 & 106 & 115 & 0.0077 \\
\hline Other fuel & 100 & 109 & 99 & 96 & 97 & 98 & 118 & 113 & 0.0232 \\
\hline Wood & 100 & 109 & 107 & 111 & 126 & 130 & 136 & 151 & 0.0464 \\
\hline \multicolumn{10}{|l|}{ Other } \\
\hline Furnishings & 100 & 108 & 127 & 127 & 158 & 171 & 184 & 191 & 0.0624 \\
\hline Personal care & 100 & 88 & 96 & 112 & 88 & 88 & 150 & 165 & 0.0892 \\
\hline Petroleum & 100 & 116 & 120 & 111 & 102 & 160 & 160 & 170 & 0.0267 \\
\hline Weighted Index & 100 & 101 & 123 & 135 & 120 & 117 & 135 & 144 & 1.0000 \\
\hline
\end{tabular}

Sources: GOA Central Statistical Office and World Bank mission estimates; cited from "Afghanistan: The Journey to Economic Development", Vol. II, March 1978, World Bank. 
Table A IV - 5. Consumer Price Index

\begin{tabular}{|c|c|c|c|c|c|c|c|}
\hline $\begin{array}{l}\text { Year Ended } \\
\text { March } 20\end{array}$ & $\begin{array}{l}\text { Total } \\
\text { Index }\end{array}$ & Cereals & Meats & $\begin{array}{l}\text { Fruits } \\
\text { and } \\
\text { Nuts }\end{array}$ & Vegetables & $\begin{array}{l}\text { Other } \\
\text { Food } \\
\text { Items }\end{array}$ & $\begin{array}{l}\text { Non- } \\
\text { Food } \\
\text { Items }\end{array}$ \\
\hline
\end{tabular}

Index Number $(1978 / 79=100)$

$\begin{array}{llllllll}1981 / 82 & 142.6 & 131.5 & 163.6 & 158.2 & 145.1 & 108.2 & 149.6 \\ 1982 / 83 & 165.7 & 159.8 & 203.4 & 176.4 & 193.0 & 128.6 & 153.4 \\ 1983 / 84 & 201.1 & 191.3 & 233.0 & 206.3 & 225.7 & 201.1 & 182.4 \\ 1984 / 85 & 241.3 & 233.9 & 271.4 & 247.7 & 287.3 & 221.9 & 221.3 \\ 1985 / 86 & 260.1 & 248.9 & 264.5 & 290.6 & 354.4 & 207.9 & 239.6 \\ 1986 / 87 & 280.9 & 317.7 & 314.2 & 326.6 & 350.1 & 220.7 & 246.5\end{array}$

Percentage change on the previous year

$\begin{array}{rrrrrrrr}1981 / 82 & 18.8 \% & 11.4 \% & 31.5 \% & 23.0 \% & 34.0 \% & 21.0 \% & 17.3 \% \\ 1982 / 83 & 16.2 \% & 21.5 \% & 24.3 \% & 11.5 \% & 33.0 \% & 18.9 \% & 2.5 \% \\ 1983 / 84 & 21.4 \% & 19.7 \% & 14.6 \% & 17.0 \% & 16.9 \% & 56.4 \% & 18.9 \% \\ 1984 / 85 & 20.0 \% & 22.3 \% & 16.5 \% & 20.1 \% & 27.3 \% & 10.3 \% & 21.3 \% \\ 1985 / 86 & 7.8 \% & 6.4 \% & -2.5 \% & 17.3 \% & 23.4 \% & -6.3 \% & 8.3 \% \\ 1986 / 87 & 8.0 \% & 27.6 \% & 18.8 \% & 12.4 \% & -1.2 \% & 6.2 \% & 2.9 \%\end{array}$

Source: GOA Central Statistical Office; cited from UNDP Discussion Paper on Afghanistan of April 1988. 
Table A IV-4. Kabul Consumer Price Index [a]

$(1978 / 79=100)$

\begin{tabular}{|c|c|c|c|c|c|c|c|c|c|}
\hline & $1977 / 78$ & $1978 / 79$ & $1979 / 80$ & $1980 / 81$ & $1981 / 82$ & $1982 / 83$ & $1983 / 84$ & $1984 / 85$ & $1985 / 86$ \\
\hline \multicolumn{10}{|l|}{ Index Numbers } \\
\hline Total Index [b] & 95.1 & 100.0 & 109.9 & 123.8 & 150.3 & 175.2 & 209.5 & 266.9 & 292.8 \\
\hline Cereals & 91.0 & 100.0 & 117.8 & 119.8 & 133.9 & 157.2 & 171.2 & 264.2 & 268.2 \\
\hline Meats & 106.3 & 100.0 & 102.1 & 121.3 & 171.6 & 230.1 & 251.3 & 280.7 & 286.0 \\
\hline Fruits and nuts & 93.7 & 100.0 & 106.6 & 103.9 & 123.5 & 163.3 & 190.9 & 243.4 & 268.7 \\
\hline Vegetables & 96.3 & 100.0 & 110.1 & 107.7 & 169.2 & 195.4 & 244.4 & 278.4 & 427.9 \\
\hline Other food items & 100.1 & 100.0 & 100.0 & 100.7 & 108.0 & 120.2 & 190.9 & 195.0 & 188.2 \\
\hline Non-food items & 102.6 & 100.0 & 111.8 & 139.2 & 161.5 & 176.2 & 206.7 & 269.6 & 287.7 \\
\hline \multicolumn{10}{|c|}{$\begin{array}{l}\text { Percentage change over } \\
\text { period of preceding year }\end{array}$} \\
\hline Index & -- & 5.2 & 9.9 & 12.6 & 21.5 & 16.6 & 19.6 & 27.4 & 9.7 \\
\hline Cereals & -- & 9.1 & 17.8 & 1.7 & 11.8 & 17.4 & 8.9 & 54.3 & 1.5 \\
\hline Meats & -- & -5.9 & 2.1 & 18.8 & 41.5 & 34.1 & 9.2 & 11.7 & 1.9 \\
\hline Fruits and nuts & -- & 6.7 & 6.6 & -2.5 & 18.9 & 32.2 & 16.9 & 27.5 & 10.4 \\
\hline Vegetables & -- & 3.8 & 10.1 & 2.2 & 57.1 & 15.5 & 25.1 & 13.9 & 53.7 \\
\hline Other food items & -- & -0.1 & -- & 0.7 & 7.2 & 11.3 & 58.8 & 2.1 & -3.5 \\
\hline Non-food items & -- & -2.6 & 11.8 & 24.5 & 16.0 & 9.1 & 17.3 & 30.4 & 6.7 \\
\hline
\end{tabular}

[a] Includes a total of 50 items from 1978/79. The original index covered 24 items with a reference base of $1968 / 69$. The old and new indexes were linked in $1978 / 79$ for data from $1977 / 78-1980 / 81$.

[b] Index includes a total of 47 items for data from 1981/82-1985/86.

Source: GOA Central Statistical Office; cited from Afghanistan: Recent Economic Developments, IMF, $1983-86$. 
Table A IV-3. Interest Rates on Bank Deposits and Loans

(in percent)

\begin{tabular}{|c|c|c|c|c|c|c|c|c|}
\hline Yr. ended March 20 & $1978 / 79$ & $1979 / 80$ & $1980 / 81$ & $1981 / 82$ & $1982 / 83$ & $1983 / 84$ & $1984 / 85$ & $1985 / 86$ \\
\hline \multicolumn{9}{|l|}{ Da Afghanistan Bank } \\
\hline Savings Deposits & 9 & 9 & 9 & 9 & 9 & 9 & 9 & 9 \\
\hline Loans to Banks & $2-9$ & $5-12$ & $2-9$ & $2-9$ & $2-9$ & $2-9$ & $2-4$ & $2-4$ \\
\hline Other Loans & $3-12$ & $5-12$ & $3-12$ & $6-12$ & $6-12$ & $6-12$ & $6-12$ & $6-12$ \\
\hline \multicolumn{9}{|l|}{ Commercial Banks } \\
\hline Savings Deposits & 9 & 9 & 9 & 9 & 9 & 9 & 9 & 9 \\
\hline Loans to Customers & $3-12$ & $5-12$ & $12-13$ & $12-13$ & $12-13$ & $12-13$ & $12-13$ & $12-13$ \\
\hline \multicolumn{9}{|c|}{$\begin{array}{l}\text { Da Afghanistan Bank and } \\
\text { Commercial Banks }\end{array}$} \\
\hline \multicolumn{9}{|c|}{ Time Deposits } \\
\hline 3 months & 4 & 4 & 4 & 4 & 4 & 4 & 4 & 4 \\
\hline 6 months & 9 & 9 & 6 & 6 & 6 & $6:$ & 6 & 6 \\
\hline 12 months & 10 & 10 & 10 & 10 & 10 & 10 & 10 & 10 \\
\hline
\end{tabular}

Source: Da Afghanistan Bank; cited from "Afghanistan Recent Economic Developments", IMF, 1983, 1986. 
Table A IV-2. Changes in Monetary Aggregates: Factors Affecting Changes in Domestic Liquidity (millions of afghanis, unless otherwise indicated)

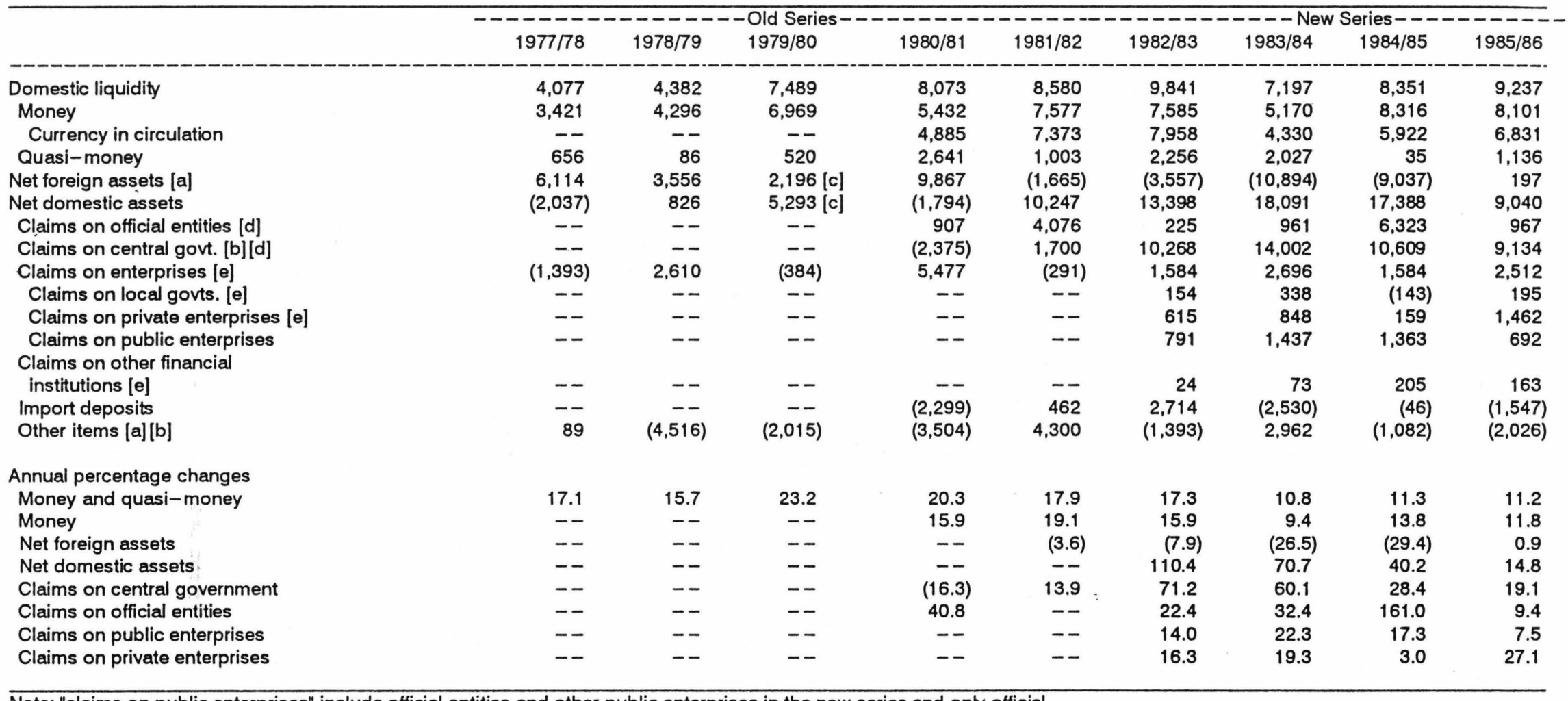

Note: "claims on public enterprises" include official entities and other public enterprises in the new series and only official

entities in the old series, other public enterprises are included under the private sector in the old series. "claims on private

enterprises" include all sectors except national government and entities under the old series. Under the new series this heading

includes local government, private sector, and other financial institutions.

[a] The change in net foreign assets in this table is equivalent to the overall balance in the balance of payments. Any

discrepancies between the change in net foreign assets in this table and the change in the stock of net foreign assets in the monetary survey are accounted for by valuation and other factors and are reflected in "other items net"

[b] Silver was revalued by Af 1394 million in 1982/83. This amount was credited to the government with the offsetting entry in

unclassified items, in the accounts of the central bank. Adjustments have been made to remove these transactions in this table.

[c] Adjusted for gold revaluation profit of Af 10,579 million.

[d] Claims on official entities are included in claims on the central government in the old series in the balance sheets of the

central bank and the commercial bank. In the monetary survey and in this table, independent information on changes in claims on

official entities have been used to provide data for 1980/81 and 1981/82, and compensating adjustments have been made to changes in

claims on Central Government.

[e] Claims on local governments, public enterprises other than official entities, and other financial institutions are included in

claims on private enterprises in the old series.

Source: GOA Da Afghanistan Bank; cited from Afghanistan: Recent Economic Developments, IMF, 1983-86. 
Table A IV-1. Absolute Values of Monetary Aggregates

(millions of afghanis)

\begin{tabular}{|c|c|c|c|c|c|}
\hline 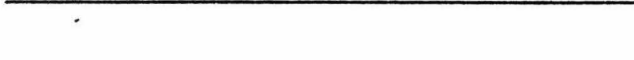 & $1978 / 79$ & $1982 / 83$ & $1983 / 84$ & $1984 / 85$ & $1985 / 86$ \\
\hline $\begin{array}{l}\text { Foreign Assets (net) } \\
\text { Domestic Credit }\end{array}$ & -- & 41,747 & 30,707 & 21,382 & 23,216 \\
\hline - claims on Government (net) & -- & 23,295 & 37,292 & 47,906 & 57,040 \\
\hline - claims on Gov. Entities [a] & -- & 9,638 & 12,374 & 19,917 & 21,771 \\
\hline - claims on private sector [b] & -- & 5,346 & 6,267 & 6,631 & 8,256 \\
\hline Money (M1): of which & 27,223 & 55,267 & 60,437 & 68,753 & 76,854 \\
\hline Quasi-Money & 4,593 & 11,457 & 13,484 & 13,519 & 14,655 \\
\hline Domestic Liquidity (M2) & 31,816 & 66,724 & 73,921 & 82,272 & 91,509 \\
\hline Memorandum Items & & " & & & \\
\hline percentage change in $\mathrm{M} 1 /$ year & 18.7 & 16.0 & 9.3 & 13.8 & 11.8 \\
\hline $\begin{array}{l}\text { percentage change in } \mathrm{M} 2 / \text { year } \\
\text { percentage change in consumer }\end{array}$ & 16.3 & 17.3 & 10.8 & 11.3 & 11.2 \\
\hline price index $(1978 / 79=100)$ & -- & 16.0 & 21.4 & 20.0 & 7.8 \\
\hline
\end{tabular}

[a] Includes claims on local governments and non-financial public enterprises.

[b] Includes claims on private sector and other financial institutions.

Source: GOA Da Afghanistan Bank; cited from the UNDP Discussion Paper on Afghanistan of April 1988. 
IV. MONETARY AND FINANCIAL STATISTICS

1. Absolute Values of Monetary Aggregates

2. Factors Affecting Changes in Domestic Liquidity

3. Interest Rates on Loans and Deposits

4. Kabul Consumer Price Index (IMF Series)

5. Consumer Price Index (UNDP Series)

6. Major Consumer Goods Price Indices (IBRD Series)

7. Survey of Average Wheat Prices (Northern Provinces)

8. Survey of Average Wheat Prices (Eastern Provinces)

O. RETURN TO MAIN MENU 
Table A IV-1. Absolute Values of Monetary Aggregates

(millions of afghanis)

\begin{tabular}{|c|c|c|c|c|c|}
\hline . & $1978 / 79$ & $1982 / 83$ & $1983 / 84$ & $1984 / 85$ & $1985 / 86$ \\
\hline $\begin{array}{l}\text { Foreign Assets (net) } \\
\text { Domestic Credit }\end{array}$ & -- & 41,747 & 30,707 & 21,382 & 23,216 \\
\hline - claims on Government (net) & -- & 23,295 & 37,292 & 47,906 & 57,040 \\
\hline - claims on Gov. Entities [a] & -- & 9,638 & 12,374 & 19,917 & 21,771 \\
\hline - claims on private sector [b] & -- & 5,346 & 6,267 & 6,631 & 8,256 \\
\hline Money (M1): of which & 27,223 & 55,267 & 60,437 & 68,753 & 76,854 \\
\hline Quasi-Money & 4,593 & 11,457 & 13,484 & 13,519 & 14,655 \\
\hline Domestic Liquidity (M2) & 31,816 & 66,724 & 73,921 & 82,272 & 91,509 \\
\hline \multicolumn{6}{|l|}{ Memorandum Items } \\
\hline percentage change in $\mathrm{M} 1 /$ year & 18.7 & 16.0 & 9.3 & 13.8 & 11.8 \\
\hline $\begin{array}{l}\text { percentage change in } \mathrm{M} 2 / \text { year } \\
\text { percentage change in consumer }\end{array}$ & 16.3 & 17.3 & 10.8 & 11.3 & 11.2 \\
\hline price index $(1978 / 79=100)$ & -- & 16.0 & 21.4 & 20.0 & 7.8 \\
\hline
\end{tabular}

[a] Includes claims on local governments and non-financial public enterprises.

[b] Includes claims on private sector and other financial institutions.

Source: GOA Da Afghanistan Bank; cited from the UNDP Discussion Paper on Afghanistan of April 1988. 
Table A IV-2. Changes in Monetary Aggregates: Factors Affecting Changes in Domestic Liquidity (millions of afghanis, unless otherwise indicated)

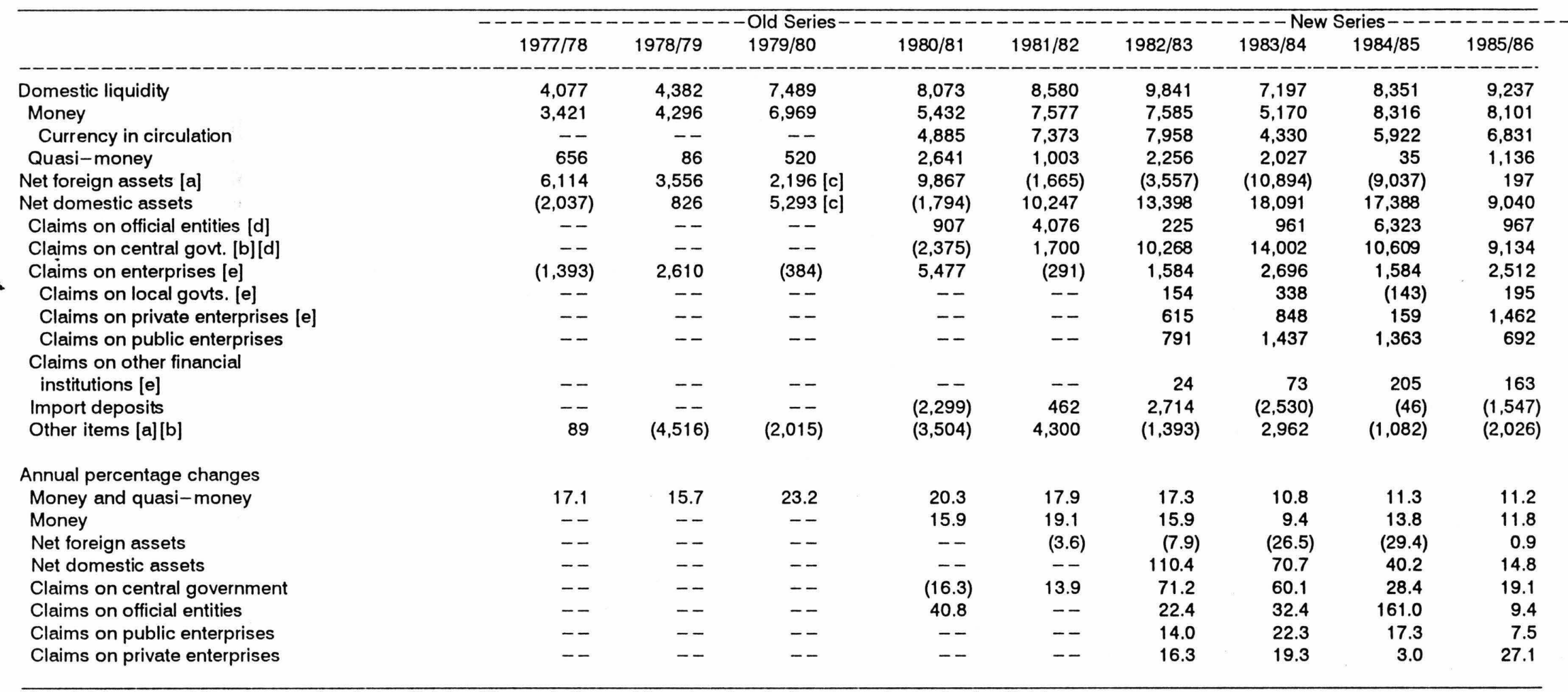

Note: "claims on public enterprises" include official entities and other public enterprises in the new series and only official

entities in the old series, other public enterprises are included under the private sector in the old series. "claims on private

enterprises" include all sectors except national government and entities under the old series. Under the new series this heading

includes local government, private sector, and other financial institutions.

[a] The change in net foreign assets in this table is equivalent to the overall balance in the balance of payments. Any

discrepancies between the change in net foreign assets in this table and the change in the stock of net foreign assets in the monetary survey are accounted for by valuation and other factors and are reflected in "other items net"

[b] Silver was revalued by Af 1394 million in 1982/83. This amount was credited to the government with the offsetting entry in

unclassified items, in the accounts of the central bank. Adjustments have been made to remove these transactions in this table.

[c] Adjusted for gold revaluation profit of Af 10,579 million.

[d] Claims on official entities are included in claims on the central government in the old series in the balance sheets of the

central bank and the commercial bank. In the monetary survey and in this table, independent information on changes in claims on

official entities have been used to provide data for 1980/81 and 1981/82, and compensating adjustments have been made to changes in claims on Central Government.

[e] Claims on local governments, public enterprises other than official entities, and other financial institutions are included in

claims on private enterprises in the old series.

Source: GOA Da Afghanistan Bank; cited from Afghanistan: Recent Economic Developments, IMF, 1983-86. 
Table A IV - 3. Interest Rates on Bank Deposits and Loans

(in percent)

\begin{tabular}{|c|c|c|c|c|c|c|c|c|}
\hline Yr. ended March 20 & $1978 / 79$ & $1979 / 80$ & $1980 / 81$ & $1981 / 82$ & $1982 / 83$ & $1983 / 84$ & $1984 / 85$ & $1985 / 86$ \\
\hline Savings Deposits & 9 & 9 & 9 & 9 & 9 & 9 & 9 & 9 \\
\hline Other Loans & $3-12$ & $5-12$ & $3-12$ & $6-12$ & $6-12$ & $6-12$ & $6-12$ & $6-12$ \\
\hline \multicolumn{9}{|l|}{ Commercial Banks } \\
\hline \multicolumn{9}{|c|}{$\begin{array}{l}\text { Da Afghanistan Bank and } \\
\text { Commercial Banks }\end{array}$} \\
\hline \multicolumn{9}{|c|}{ Time Deposits } \\
\hline 3 months & 4 & 4 & 4 & 4 & 4 & 4 & 4 & 4 \\
\hline 6 months & 9 & 9 & 6 & 6 & 6 & 6 & 6 & 6 \\
\hline
\end{tabular}

Source: Da Afghanistan Bank; cited from "Afghanistan Recent Economic Developments", IMF, 1983, 1986. 
Table A IV-4. Kabul Consumer Price Index [a]

$(1978 / 79=100)$

\begin{tabular}{|c|c|c|c|c|c|c|c|c|c|}
\hline & $1977 / 78$ & $1978 / 79$ & $1979 / 80$ & $1980 / 81$ & $1981 / 82$ & $1982 / 83$ & $1983 / 84$ & $1984 / 85$ & $1985 / 86$ \\
\hline \multicolumn{10}{|l|}{ Index Numbers } \\
\hline Total Index [b] & 95.1 & 100.0 & 109.9 & 123.8 & 150.3 & 175.2 & 209.5 & 266.9 & 292.8 \\
\hline Cereals & 91.0 & 100.0 & 117.8 & 119.8 & 133.9 & 157.2 & 171.2 & 264.2 & 268.2 \\
\hline Meats & 106.3 & 100.0 & 102.1 & 121.3 & 171.6 & 230.1 & 251.3 & 280.7 & 286.0 \\
\hline Fruits and nuts & 93.7 & 100.0 & 106.6 & 103.9 & 123.5 & 163.3 & 190.9 & 243.4 & 268.7 \\
\hline Vegetables & 96.3 & 100.0 & 110.1 & 107.7 & 169.2 & 195.4 & 244.4 & 278.4 & 427.9 \\
\hline Other food items & 100.1 & 100.0 & 100.0 & 100.7 & 108.0 & 120.2 & 190.9 & 195.0 & 188.2 \\
\hline Non-food items & 102.6 & 100.0 & 111.8 & 139.2 & 161.5 & 176.2 & 206.7 & 269.6 & 287.7 \\
\hline \multicolumn{10}{|c|}{$\begin{array}{l}\text { Percentage change over } \\
\text { period of preceding year }\end{array}$} \\
\hline Index & -- & 5.2 & 9.9 & 12.6 & 21.5 & 16.6 & 19.6 & 27.4 & 9.7 \\
\hline Cereals & -- & 9.1 & 17.8 & 1.7 & 11.8 & 17.4 & 8.9 & 54.3 & 1.5 \\
\hline Meats & -- & -5.9 & 2.1 & 18.8 & 41.5 & 34.1 & 9.2 & 11.7 & 1.9 \\
\hline Fruits and nuts & -- & 6.7 & 6.6 & -2.5 & 18.9 & 32.2 & 16.9 & 27.5 & 10.4 \\
\hline Vegetables & -- & 3.8 & 10.1 & 2.2 & 57.1 & 15.5 & 25.1 & 13.9 & 53.7 \\
\hline Other food items & -- & -0.1 & -- & 0.7 & 7.2 & 11.3 & 58.8 & 2.1 & -3.5 \\
\hline Non-food items & -- & -2.6 & 11.8 & 24.5 & 16.0 & 9.1 & 17.3 & 30.4 & 6.7 \\
\hline
\end{tabular}

[a] Includes a total of 50 items from 1978/79. The original index covered 24 items with a reference base of $1968 / 69$. The old and new indexes were linked in $1978 / 79$ for data from $1977 / 78-1980 / 81$.

[b] Index includes a total of 47 items for data from 1981/82-1985/86.

Source: GOA Central Statistical Office; cited from Afghanistan: Recent Economic Developments, IMF, $1983-86$. 
Table A IV-5. Consumer Price Index

\begin{tabular}{|c|c|c|c|c|c|c|c|}
\hline $\begin{array}{l}\text { Year Ended } \\
\text { March } 20\end{array}$ & $\begin{array}{l}\text { Total } \\
\text { Index }\end{array}$ & Cereals & Meats & $\begin{array}{l}\text { Fruits } \\
\text { and } \\
\text { Nuts }\end{array}$ & Vegetables & $\begin{array}{l}\text { Other } \\
\text { Food } \\
\text { Items }\end{array}$ & $\begin{array}{l}\text { Non- } \\
\text { Food } \\
\text { Items }\end{array}$ \\
\hline
\end{tabular}

Index Number $(1978 / 79=100)$

$\begin{array}{llllllll}1981 / 82 & 142.6 & 131.5 & 163.6 & 158.2 & 145.1 & 108.2 & 149.6 \\ 1982 / 83 & 165.7 & 159.8 & 203.4 & 176.4 & 193.0 & 128.6 & 153.4 \\ 1983 / 84 & 201.1 & 191.3 & 233.0 & 206.3 & 225.7 & 201.1 & 182.4 \\ 1984 / 85 & 241.3 & 233.9 & 271.4 & 247.7 & 287.3 & 221.9 & 221.3 \\ 1985 / 86 & 260.1 & 248.9 & 264.5 & 290.6 & 354.4 & 207.9 & 239.6 \\ 1986 / 87 & 280.9 & 317.7 & 314.2 & 326.6 & 350.1 & 220.7 & 246.5\end{array}$

Percentage change on the previous year

$\begin{array}{rrrrrrrr}1981 / 82 & 18.8 \% & 11.4 \% & 31.5 \% & 23.0 \% & 34.0 \% & 21.0 \% & 17.3 \% \\ 1982 / 83 & 16.2 \% & 21.5 \% & 24.3 \% & 11.5 \% & 33.0 \% & 18.9 \% & 2.5 \% \\ 1983 / 84 & 21.4 \% & 19.7 \% & 14.6 \% & 17.0 \% & 16.9 \% & 56.4 \% & 18.9 \% \\ 1984 / 85 & 20.0 \% & 22.3 \% & 16.5 \% & 20.1 \% & 27.3 \% & 10.3 \% & 21.3 \% \\ 1985 / 86 & 7.8 \% & 6.4 \% & -2.5 \% & 17.3 \% & 23.4 \% & -6.3 \% & 8.3 \% \\ 1986 / 87 & 8.0 \% & 27.6 \% & 18.8 \% & 12.4 \% & -1.2 \% & 6.2 \% & 2.9 \%\end{array}$

Source: GOA Central Statistical Office; cited from UNDP Discussion Paper on

Afghanistan of April 1988. 
Table A IV -6. Major Consumer Goods Price Indices $(1968 / 69=100)$

\begin{tabular}{|c|c|c|c|c|c|c|c|c|c|}
\hline & $1968 / 69$ & $1969 / 70$ & $1970 / 71$ & $1971 / 72$ & $1972 / 73$ & $1973 / 74$ & $1974 / 75$ & \multicolumn{2}{|c|}{$1975 / 76$ Weights } \\
\hline \multicolumn{10}{|l|}{ Food } \\
\hline Cereals & 100 & 95 & 135 & 161 & 124 & 95 & 115 & 127 & 0.4167 \\
\hline Meat & 100 & 106 & 112 & 108 & 124 & 147 & 178 & 185 & 0.0767 \\
\hline Fruit & 100 & 115 & 134 & 139 & 121 & 161 & 171 & 150 & 0.0760 \\
\hline Vegetables & 100 & 95 & 120 & 115 & 107 & 144 & 134 & 147 & 0.0409 \\
\hline Other food & 100 & 97 & 100 & 110 & 140 & 139 & 143 & 174 & 0.0366 \\
\hline Clothing & 100 & 117 & 119 & 119 & 117 & 121 & 118 & 122 & 0.0779 \\
\hline Rent & 100 & 100 & 100 & 100 & 100 & 100 & 100 & 100 & 0.0196 \\
\hline \multicolumn{10}{|l|}{ Fuel \& Electricity } \\
\hline Electricity & 100 & 108 & 116 & 114 & 108 & 112 & 106 & 115 & 0.0077 \\
\hline Other fuel & 100 & 109 & 99 & 96 & 97 & 98 & 118 & 113 & 0.0232 \\
\hline Wood & 100 & 109 & 107 & 111 & 126 & 130 & 136 & 151 & 0.0464 \\
\hline \multicolumn{10}{|l|}{ Other } \\
\hline Furnishings & 100 & 108 & 127 & 127 & 158 & 171 & 184 & 191 & 0.0624 \\
\hline Personal care & 100 & 88 & 96 & 112 & 88 & 88 & 150 & 165 & 0.0892 \\
\hline Petroleum & 100 & 116 & 120 & 111 & 102 & 160 & 160 & 170 & 0.0267 \\
\hline Weighted Index & 100 & 101 & 123 & 135 & 120 & 117 & 135 & 144 & 1.0000 \\
\hline
\end{tabular}

Sources: GOA Central Statistical Office and World Bank mission estimates; cited from "Afghanistan: The Journey to Economic Development", Vol. II, March 1978, World Bank. 
Table A IV-8. Survey of Average Wheat

Prices in Eastern Provinces

(afghanis per seer)

\begin{tabular}{lrrrr}
\hline & Apr/May & Apr/May & Winter & Apr/May \\
Province & 1985 & 1986 & $1986 / 87$ & 1987 \\
\hline Kabul & 125 & 170 & 225 & 260 \\
Kabul City & 165 & 205 & 250 & 270 \\
Kapisa/Parvan & 100 & 150 & -- & 260 \\
Vardak & 145 & 185 & 280 & 265 \\
Lowgar & 120 & 165 & 375 & 235 \\
Ghazni & 140 & 190 & 305 & 235 \\
Paktia & 140 & 200 & 400 & 260 \\
Nangarhar & 100 & 150 & -- & 220 \\
Laghman & 110 & 160 & -- & 250 \\
Kanorha & -- & -- & 320 & -- \\
Bamian & 150 & 180 & & 300 \\
& & & &
\end{tabular}

Note: One seer is equal to approximately seven kilograms.

Source: Confidential Crossborder Survey, 1987; cited from the Orkand Report. 
Table A IV-7. Survey of Average Wheat

Prices in Northern Provinces

(afghanis per seer)

\begin{tabular}{lrrrr}
\hline & Apr/May & Apr/May & Winter & Apr/May \\
Province & 1985 & 1986 & $1986 / 87$ & 1987 \\
\hline Konduz & 115 & 150 & 265 & 180 \\
Takhar & 115 & 150 & 285 & 200 \\
Baghlan & 130 & 180 & 345 & 235 \\
Balkh & 115 & 180 & 325 & 265 \\
Badakhshar & 170 & 170 & 300 & 270 \\
Samangan & 125 & 205 & 450 & 355 \\
Faryab & 175 & 325 & 450 & 395 \\
Badghisat & 250 & 335 & 395 & 410 \\
Jowzjan & 185 & 290 & 450 & 410 \\
& & & & \\
\hline
\end{tabular}

Note: One seer is equal to approximately seven kilograms.

Source: A.I.D. survey, 1987, untitled, unpublished; cited from the Orkand Report. 
1. Indicators of Dev. in Ext. Sector

2. IMF Summary of BOP Breakdown

3. IMF Summary of Calendar Year BOP

4. IMF Summary of Bilateral BOP

5. IMF Summary of Multilateral BOP

6. IBRD Summary of BOP Breakdown

7. Ext. Public Debt and Svc. Pmts.

8. Ext. Debt Svc. (Total Debt)

9. Ext. Debt Svc. (Suppliers Credits)

10. External Public Debt Outstanding

11. Int'I Reserves and Related Items

12. Exports by Commodity (IMF)
13. Mdse. Exports by Commodity

14. Commodity Exports (IBRD)

15. Exports by Region

16. Mdse. Exports (7-yr. Plan)

17. Major Export Qty. Indices

18. Value of Mdse. Trade (IMF)

19. Mdse. Imports by Category

20. Official Imports from Pak.

21. Imports by Commodity

22. Imports by Region

23. Exchange Rates ('73-'89)

O. RETURN TO MAIN MENU 
(housands of U.S. dollars)

\begin{tabular}{|c|c|c|c|c|}
\hline & $1978 / 79$ & 1984/85 & 1985/B6 & $\begin{array}{r}1986 / 87 \\
\text { (prellim.) }\end{array}$ \\
\hline \multicolumn{5}{|l|}{$\begin{array}{l}\text { Foreign trade (commerdal) } \\
\text { Soviet Union }\end{array}$} \\
\hline Exports (fob), of which & 112,322 & 371,458 & 383,562 & 380,099 \\
\hline $\begin{array}{l}\text { Natural gas } \\
\text { Imports (cif) }\end{array}$ & $\begin{array}{l}53.018 \\
(89512)\end{array}$ & $\begin{array}{l}314,326 \\
(614,455)\end{array}$ & $\begin{array}{l}309,387 \\
(556,896)\end{array}$ & $\begin{array}{l}259,579 \\
465,477\end{array}$ \\
\hline Trade balance & 22,810 & $(242,997)$ & $\begin{array}{l}(173,334) \\
(173)\end{array}$ & $(85,378)$ \\
\hline \multicolumn{5}{|l|}{ Other Countries } \\
\hline Exports (tob) & 210,451 & 261,420 & 183,269 & 171,819 \\
\hline Imports (cin) & $\begin{array}{l}(330,413) \\
(119,962)\end{array}$ & $\begin{array}{l}(483,234) \\
(221,814)\end{array}$ & $\begin{array}{l}(326,159) \\
(142,890)\end{array}$ & $\begin{array}{l}(396,229) \\
(224,410)\end{array}$ \\
\hline \multirow{2}{*}{\multicolumn{5}{|c|}{ Total trade }} \\
\hline & & & & \\
\hline Imports (cif) & 419925 & $\begin{array}{l}052.878 \\
1097689\end{array}$ & 883055 & $\begin{array}{l}551,918 \\
861,706\end{array}$ \\
\hline Trade Balance & $(97,152)$ & $(464,811)$ & $(316,224)$ & $(309,788)$ \\
\hline \multicolumn{5}{|l|}{ Foreign aid } \\
\hline $\begin{array}{l}\text { Project aid } \\
\text { Non-project aid }\end{array}$ & $\begin{array}{l}170,000 \\
65,000\end{array}$ & $\begin{array}{l}162,000 \\
125,000\end{array}$ & $\begin{array}{l}165,000 \\
146,000\end{array}$ & $\begin{array}{l}170,000 \\
159,000\end{array}$ \\
\hline \multirow{2}{*}{\multicolumn{5}{|c|}{ Debt Repayments }} \\
\hline & 43,000 & 107,000 & 67,000 & \\
\hline Interest & 15,000 & 18,000 & 9,000 & 10,000 \\
\hline \multicolumn{5}{|c|}{$\begin{array}{l}\text { Gross Reserves of Central Bank } \\
\text { excludine gold. }\end{array}$} \\
\hline $\begin{array}{l}\text { exclualing gold: } \\
\text { Convertible Currency } \\
\text { Inconvertible Currency }\end{array}$ & $\begin{array}{r}420,000 \\
7,000\end{array}$ & 229,000 & 296,000 & 258,000 \\
\hline \multirow{2}{*}{\multicolumn{5}{|c|}{$\begin{array}{l}\text { Memorandum Items } \\
\text { Exchange Rate -- atghanis to U.S\$ }\end{array}$}} \\
\hline & & & & \\
\hline Central Bank & 45.3 & 50.6 & 50.6 & 50.6 \\
\hline
\end{tabular}

Table A V-1. Indicators of Developments in External Sector

(thousands of U.S. dollars)

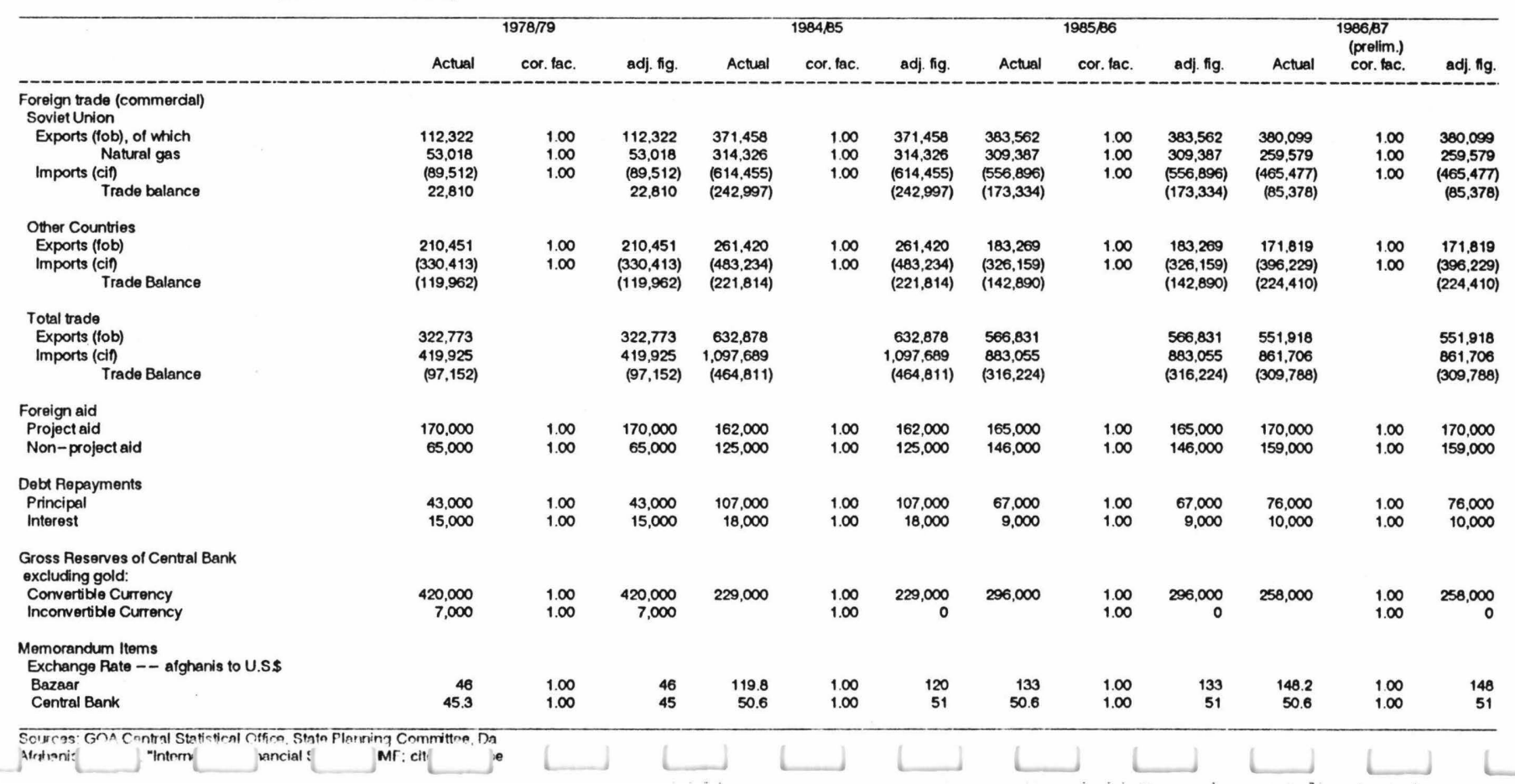


Table A V -2. IMF Summary of Balance of Payments Breakdown

(millions of U.S. dollers)

\begin{tabular}{|c|c|c|c|c|c|c|c|c|c|}
\hline & $1977 / 78$ & 1978/79 & 1979/80 & $1980 / 81$ & $1981 / 82$ & $1982 / 83$ & $1903 / 84$ & $1984 / 85$ & $1985 / 80$ \\
\hline 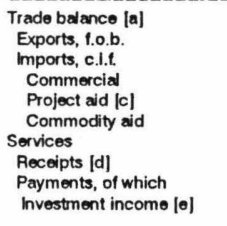 & $\begin{array}{c}(196.3) \\
326.7 \\
(523.0) \\
(361.1) \\
(141.6) \\
(20.3) \\
67.5 \\
-- \\
- \\
(6.5)\end{array}$ & $\begin{array}{c}(202.9) \\
330.7 \\
(629.6) \\
(453.2) \\
(145.5) \\
(30.9) \\
67.8 \\
-- \\
-- \\
5.7\end{array}$ & $\begin{aligned}(183.1) \\
481.2 \\
(684.3) \\
(424.3) \\
(187.4) \\
(55.6) \\
47.7 \\
--. \\
--. \\
23.2\end{aligned}$ & $\begin{array}{l}(111.1) \\
713.5 \\
(832.6) \\
(535.2) \\
(171.6) \\
(125.6) \\
80.8 \\
-- \\
-- \\
45.0\end{array}$ & $\begin{array}{c}(148.2) \\
838.11 \mathrm{bl} \\
(1,031.3) \\
(70.2) \\
(146.2) \\
(88.9) \\
22.0 \\
99.0 \\
(77.0) \\
(3.5)\end{array}$ & $\begin{array}{l}(230.1) \\
743.8 \\
(973.9) \\
(710.1) \\
(174.2) \\
(73.6) \\
70.0 \\
151.7 \\
(45.7) \\
(20.1)\end{array}$ & $\begin{array}{l}(220.4) \\
628.7 \\
(025.1) \\
(717.2) \\
(145.3) \\
(62.6) \\
46.8 \\
88.0 \\
(41.2) \\
(21.4)\end{array}$ & $\begin{array}{c}(067.9) \\
047.8 \\
(1,35.4) \\
(1,02.5) \\
(162.09 \\
(124.9) \\
25.9 \\
60.7 \\
(34.8) \\
(18.5)\end{array}$ & 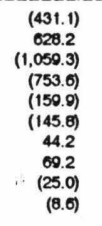 \\
\hline Current Account & (128.8) & $(225.1)$ & (135.4) & (38.3) & (120.2) & (180.1) & (249.6) & $(641.7)$ & $(380.9)$ \\
\hline $\begin{array}{l}\text { Nonmonotary capital } \\
\text { Receipts } \\
\text { Public loans } \\
\text { Suppliers' credits } \\
\text { Grants }[\mid] \\
\text { Repayments }\end{array}$ & $\begin{array}{l}181.1 \\
218.4 \\
1777.8 \\
-10- \\
40.8 \\
(37.3)\end{array}$ & $\begin{array}{l}192.4 \\
235.2 \\
144.8 \\
7.4 \\
83.0 \\
(42.8)\end{array}$ & $\begin{array}{c}259.6 \\
317.1 \\
178.9 \\
57.9 \\
82.3 \\
(57.5)\end{array}$ & $\begin{array}{l}200.8 \\
345.6 \\
150.5 \\
48.5 \\
140.6 \\
(45.8)\end{array}$ & $\begin{array}{l}174.9 \\
272.7 \\
160.1 \\
16.7 \\
80.9 \\
(97.8)\end{array}$ & $\begin{array}{l}152.0 \\
280.6 \\
157.2 \\
27.0 \\
82.4 \\
(113.7)\end{array}$ & $\begin{array}{l}119.9 \\
218.3 \\
108.1 \\
11.2 \\
09.0 \\
198.4)\end{array}$ & $\begin{array}{l}184.3 \\
201.8 \\
98.9 \\
65.6 \\
127.3 \\
(107.5)\end{array}$ & $\begin{array}{r}221.3 \\
311.1 \\
122.0 \\
45.4 \\
143.7 \\
(89.8)\end{array}$ \\
\hline Errors and ommissions, net & 103.1 & 130.9 & $(64.9)$ & $(67.0)$ & $(80.9)$ & (63.1) & (85.6) & 278.8 & 180.5 \\
\hline $\begin{array}{l}\text { Overall balance } \\
\text { Bifaterd } \\
\text { Convertible }\end{array}$ & $\begin{array}{r}155.4 \\
14.9 \\
100.3\end{array}$ & $\begin{array}{l}98.2 \\
150.8 \\
153.0\end{array}$ & $\begin{array}{l}50.3 \\
01.3 \\
(2.0)\end{array}$ & $\begin{array}{l}194.5 \\
260.9 \\
(60.4)\end{array}$ & $\begin{array}{l}(32.2) \\
20.1 \\
(61.3)\end{array}$ & $\begin{array}{l}(70.3) \\
(73.1) \\
2.8\end{array}$ & $\begin{array}{r}(215.3) \\
(140.5) \\
(65.8)\end{array}$ & $\begin{array}{l}(178.5) \\
(251.5) \\
72.0\end{array}$ & $\begin{array}{r}3.0 \\
(24.6) \\
28.5\end{array}$ \\
\hline $\begin{array}{l}\text { Monetery movements } \\
\text { Contal bank } \\
\text { Commercial banks }\end{array}$ & $\begin{array}{r}(155.4) \\
(138.4) \\
(17.09\end{array}$ & $\begin{array}{l}(90.2) \\
(90.3) \\
(8.9)\end{array}$ & $\begin{array}{l}(50.3) \\
(40.1) \\
(13.2)\end{array}$ & $\begin{array}{r}(104.5) \\
(100.5) \\
(4.0)\end{array}$ & $\begin{array}{l}32.2 \\
40.4 \\
(8.2)\end{array}$ & $\begin{array}{l}70.3 \\
111.8 \\
(41.5)\end{array}$ & $\begin{array}{r}215.3 \\
177.9 \\
37.4\end{array}$ & $\begin{array}{l}178.6 \\
211.1 \\
(32.5)\end{array}$ & $\begin{array}{l}(3.9) \\
(2.5)|g| \\
(1.4)\end{array}$ \\
\hline
\end{tabular}

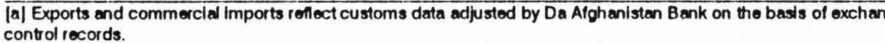

|b| Adjustod for underestimation of recelpts in the customs estimates as a net positive errors and ommissons

enty in tro biatorad badance ol parments accounc

(D)

lel includes interest on foreign debt.

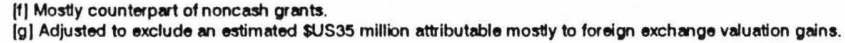

Source: GOA Da Atghanistan Bank; cited trom Atghanistan: Recent Economic Developments, IMF, 1963-86.

Table A V -2. IMF Summary of Balance of Payments Breat

(millions of U.S. dollars)

\begin{tabular}{|c|c|c|c|c|c|c|c|c|c|}
\hline & Actual & $\begin{array}{l}1977778 \\
\text { cor. fac. }\end{array}$ & adj. fig. & Actual & $\begin{array}{l}1978 / 70 \\
\text { cor. fac. adj. }\end{array}$ & Actual & $\begin{array}{l}1979 / 80 \\
\text { cor. fac. }\end{array}$ & adj. fig. & $\begin{array}{l}199 \\
\text { Actual cor. }\end{array}$ \\
\hline $\begin{array}{l}\text { Trade balance |a| } \\
\text { Exports, t.o.b. }\end{array}$ & $\begin{array}{l}\left(\begin{array}{l}190.3) \\
326.7\end{array}\right) \\
\text { (a) }\end{array}$ & 1.00 & $\begin{array}{l}\left(\begin{array}{l}196.3) \\
326.7\end{array}\right) \\
\text { (a) }\end{array}$ & $\begin{array}{l}(202.9) \\
336.7\end{array}$ & $1.00 * \ldots * *$ & $\begin{array}{l}(183.1) \\
481.2\end{array}$ & 1.00 & $\begin{array}{l}(183.1) \\
481.2\end{array}$ & $\begin{array}{l}(119.1) \\
713.5 * \ldots * *\end{array}$ \\
\hline Imports, c.l.t. & & & (523.0) & & & (6654.3) & & $(664.3)$ & \\
\hline Commercial & (361.1) & 1.00 & & $(453.2)$ & $1.00 * m * n$ & (424.3) & 1.00 & (4424.3) & (535.2) $* \cdots * *$ \\
\hline $\begin{array}{l}\text { Project aid }|y| c \mid \\
\text { Commodity idd }\end{array}$ & (141.6) & 1.00 & (141.6) & (145.5) & $1.00 * m * \pi$ & (187.4) & 1.00 & $(187.4)$ & 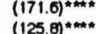 \\
\hline $\begin{array}{l}\text { Commmodity aid } \\
\text { Services }\end{array}$ & $\begin{array}{l}(20.3) \\
67.5\end{array}$ & & $\begin{array}{l}(20.3) \\
67.5\end{array}$ & $\begin{array}{l}(30.9) \\
678\end{array}$ & $1.00=$ & $\begin{array}{l}(52.6) \\
47.7\end{array}$ & & $\begin{array}{l}(52.6) \\
675\end{array}$ & 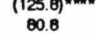 \\
\hline Recoipts $[d]$ & -- & 1.00 & &.- & 1.00 & -- & 1.00 & & $--\infty+\cdots+\infty$ \\
\hline $\begin{array}{l}\text { Payments, of which } \\
\text { Investment income |0| }\end{array}$ & -- & 1.00 & & $\overline{5}$ & 1.00 & $-\overline{232}$ & 1.00 & & $45.0 \ldots *$ \\
\hline Current Account & $(128.8)>2)$ & & (128.8) & 1225.1 & 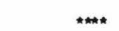 & $(135.4)$ & & (115.6) & (38.3) \\
\hline Nonmonetary capital & 181.1 & & 181.1 & 1924 & *n** & 2590 & & 259.6 & \\
\hline Roceipts & 218.4 & & 218.4 & 235.2 & $\therefore *$ & 317.1 & & & \\
\hline Public & 177.6 & 1.00 & 177.0 & 144.8 & $1.00 * \ldots+*$ & 176. & 1.00 & & $156.5 \ldots+\cdots$ \\
\hline Suppliers' credits & & 1.00 & 0.0 & 7.4 & 1.007 .4 & 57. & 1.00 & 57.9 & $48.5 \ldots \ldots$ \\
\hline Grants (i) & 40.8 & 1.00 & 40.8 & 83.0 & $1.00 \ldots$ & 82.3 & 1.00 & 82.3 & $140.6 * \cdots *$ \\
\hline Repayments & (37.3) & 1.00 & (37.3) & (42.8) & $1.00=\ldots$ & (57.5) & 1.00 & $(57.5)$ & $(45.8)^{\prime} \cdots \cdots$ \\
\hline Errors and ommissions, net & 103.1 & 1.00 & 103.1 & 130.9 & $1.00 * * n *$ & (64.9) & 1.00 & (64.9) & $(67.0)^{* \cdots \cdots}$ \\
\hline Overeall balance & & & & & *n** & 50.3 & & & \\
\hline $\begin{array}{l}\text { Bllataral } \\
\text { Convertble }\end{array}$ & $\begin{array}{c}(4.9) \\
1003\end{array}$ & $\begin{array}{l}1.00 \\
1.00\end{array}$ & $\begin{array}{c}(4.9) \\
100.3\end{array}$ & $\begin{array}{l}\text { (50.8) } \\
155.0\end{array}$ & $1.00+\cdots * *$ & $\begin{array}{l}61.3 \\
(2.0) \\
6\end{array}$ & $\begin{array}{l}1.00 \\
1.00\end{array}$ & $\begin{array}{l}80.3 \\
(2.0)\end{array}$ & 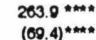 \\
\hline Monetary movements & & & (155.4 & (98.2) & & (50.3) & & (59.3) & (194.5) \\
\hline Contral bank & & 1.00 & (138.4) & $(89.3)$ & 1.00 **m**. & (46.1) & 1.00 & (46.1) & (1100.5)* \\
\hline Commercial banks & & 1.00 & $(17.0)$ & (8.9) & & (13.2) & 1.00 & & \\
\hline
\end{tabular}

[a] Exports and commercia imports reflect customs data adjustod by Da Atghanistan Bank on the basis of exch
control records.

of receipts in the customs estimates as

IVI Includes imports financed by suppliers' crecodits and public loans.

Al Includes unrequited transters, net

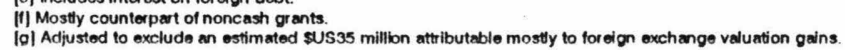


Table A V-3. IMF Summary of Calendar Year Balance of Payments

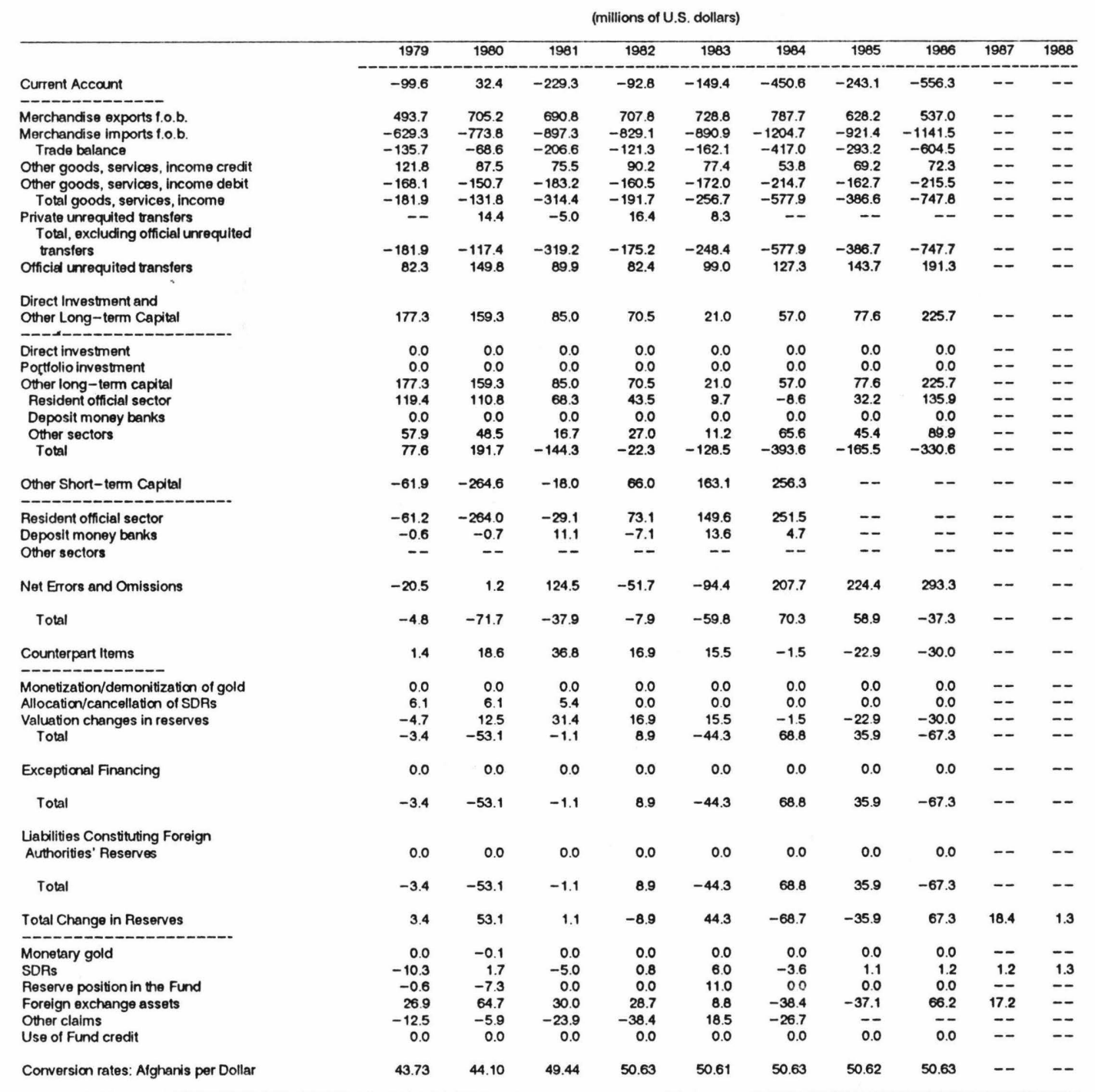

$\begin{array}{llllllllll}1.292 & 1.30153 & 1.17918 & 1.10401 & 1.069 & 1.02501 & 1.01534 & 1.17317 & 1.29307 & 1.34423\end{array}$

\begin{tabular}{|c|c|c|c|c|c|c|c|c|c|}
\hline 1979 & 1980 & 1981 & 1982 & 1983 & 1984 & 1985 & 1986 & 1987 & 1988 \\
\hline-77.1 & 24.9 & -194.5 & -84.1 & -139.8 & -439.6 & -239.4 & -474.2 & 0.0 & 0.0 \\
\hline 382.1 & 541.8 & 585.8 & 641.1 & 681.8 & 768.5 & 618.7 & 457.7 & -- & -- \\
\hline-487 & -594.5 & -761.0 & -751.0 & -833.4 & -1175.3 & -907.5 & -973.0 & -- & -- \\
\hline-105.0 & -52.7 & -175.2 & -109.9 & -151.6 & -406.8 & -288.8 & -515.3 & 0.0 & 0.0 \\
\hline 94.3 & 67.2 & 64.0 & 81.7 & 72.4 & 52.5 & 68.2 & 61.6 & -- & $=-$ \\
\hline $\begin{array}{l}-130.1 \\
-10.8\end{array}$ & $\begin{array}{l}-115.8 \\
-1013\end{array}$ & $\begin{array}{l}-155.4 \\
-2066.6\end{array}$ & $\begin{array}{l}-145.4 \\
-1736\end{array}$ & $\begin{array}{l}-160.9 \\
-2001\end{array}$ & $\begin{array}{l}-209.5 \\
-5538\end{array}$ & -160.2 & $\begin{array}{l}-183.7 \\
-6374 .\end{array}$ & $-\overline{0}$ & $\overline{0.0}$ \\
\hline $\begin{array}{l}-140.8 \\
-1\end{array}$ & $\begin{array}{r}-101.3 \\
11.1\end{array}$ & $\begin{array}{r}-2066.6 \\
-4.2\end{array}$ & $\begin{array}{r}-173.6 \\
14.9\end{array}$ & $\begin{array}{r}-240.1 \\
7.8\end{array}$ & $\begin{array}{l}-563.8 \\
-\end{array}$ & $\begin{array}{l}-380.8 \\
-\end{array}$ & -637.4 & $\begin{array}{l}0.0 \\
--\end{array}$ & \\
\hline $\begin{array}{r}-140.8 \\
63.7\end{array}$ & $\begin{array}{r}-90.2 \\
115.1\end{array}$ & $\begin{array}{r}-270.7 \\
76.2\end{array}$ & $\begin{array}{r}-158.7 \\
74.8\end{array}$ & $\begin{array}{r}-232.4 \\
92.6\end{array}$ & $\begin{array}{r}-563.8 \\
124.2\end{array}$ & $\begin{array}{r}-380.9 \\
141.5\end{array}$ & $\begin{array}{r}-637.3 \\
163.1\end{array}$ & $=-$ & $\begin{array}{l}-- \\
--\end{array}$ \\
\hline 137.2 & 122.4 & 72.1 & 63.9 & 19.8 & 55.6 & 76.4 & 192.4 & 0.0 & 0.0 \\
\hline 0.0 & 0.0 & 0.0 & 0.0 & 0.0 & 0.0 & 0.0 & 0.0 & $=-$ & $=-$ \\
\hline $\begin{array}{r}0.0 \\
137.2\end{array}$ & $\begin{array}{r}0.0 \\
122.4\end{array}$ & $\begin{array}{l}0.0 \\
72.1\end{array}$ & $\begin{array}{r}0.0 \\
63.9\end{array}$ & $\begin{array}{r}0.0 \\
19.6\end{array}$ & $\begin{array}{r}0.0 \\
55.6\end{array}$ & $\begin{array}{l}0.0 \\
76.4\end{array}$ & $\begin{array}{r}0.0 \\
192.4\end{array}$ & $\overline{0.0}$ & 0.0 \\
\hline 92.4 & 85.1 & 57.8 & 39.4 & 9.1 & -8.4 & 31.7 & 115.8 & - & - \\
\hline 0.0 & 0.0 & 0.0 & 0.0 & 0.0 & 0.0 & 0.0 & 0.0 & -- & -- \\
\hline 44.8 & 37.3 & 14.2 & 24.5 & 10.5 & & 44.7 & & - & $-\overline{0}$ \\
\hline 60.1 & 147.3 & -122.4 & -20.2 & -120.2 & -384.0 & -163.0 & -281.8 & 0.0 & \\
\hline-47.8 & -2003.3 & -15.3 & 59.8 & 152.6 & 250.0 & -- & -- & 0.0 & 0.0 \\
\hline-47.4 & -202.8 & -24.7 & 68.2 & 139.8 & 245.4 & -- & -- & -- & -- \\
\hline-0.5 & $\begin{array}{r}-0.5 \\
-\end{array}$ & ${ }_{--}^{9.4}$ & -6.4 & 7 & $\approx$ & $=-$ & $=-$ & $=$ & $=-$ \\
\hline-15.9 & 0.9 & 105.6 & -46.8 & -88.3 & 202.0 & 221.0 & 250.0 & -- & -- \\
\hline-3.7 & -55.1 & -32.1 & -7.2 & -55.8 & 68.6 & 58.0 & -31.8 & 0.0 & 0.0 \\
\hline 1.1 & 14.3 & 31.2 & 15.3 & 14.5 & -1.5 & -22.6 & -25.6 & 0.0 & 0.0 \\
\hline $\begin{array}{l}0.0 \\
4.7\end{array}$ & $\begin{array}{l}0.0 \\
4.7\end{array}$ & $\begin{array}{l}0.0 \\
4.6\end{array}$ & $\begin{array}{l}0.0 \\
0.0\end{array}$ & & $\begin{array}{l}0.0 \\
0.0\end{array}$ & $\begin{array}{l}0.0 \\
0.0\end{array}$ & $\begin{array}{l}0.0 \\
0.0\end{array}$ & $=-$ & $=-$ \\
\hline-3.6 & 9.6 & 26.6 & 15.3 & 14.5 & -1.5 & -22.6 & -25.6 & -- & $=-$ \\
\hline-2.6 & -40.8 & -0.9 & 8.1 & -41.4 & 67.1 & 35.4 & -57.4 & 0.0 & \\
\hline 0.0 & 0.0 & 0.0 & 0.0 & 0.0 & 0.0 & 0.0 & 0.0 & -- & -- \\
\hline-2.6 & -40.8 & -0.9 & 8.1 & -41.4 & 67.1 & 35.4 & -57.4 & 0.0 & 0.0 \\
\hline 0.0 & 0.0 & 0.0 & 0.0 & 0.0 & 0.0 & 0.0 & 0.0 & -- & -- \\
\hline-2.6 & -40.8 & -0.9 & 8.1 & -41.4 & 67.1 & 35.4 & -57.4 & 0.0 & 0.0 \\
\hline 2.6 & 40.8 & 0.8 & -8.1 & 41.4 & -67.0 & -35.4 & 57.4 & 14.2 & 1.0 \\
\hline $\begin{array}{r}0.0 \\
-8.0\end{array}$ & $\begin{array}{r}-0.1 \\
1.3\end{array}$ & $\begin{array}{r}0.0 \\
-4.2\end{array}$ & $\begin{array}{l}0.0 \\
0.7\end{array}$ & $\begin{array}{l}0.0 \\
5.6\end{array}$ & $\begin{array}{r}0.0 \\
-3.5\end{array}$ & $\begin{array}{l}0.0 \\
1.1\end{array}$ & $\begin{array}{l}0.0 \\
1.0\end{array}$ & $\overline{0.9}$ & $\overline{1.0}$ \\
\hline-0.5 & -5 & & & 10.3 & 0.0 & 0.0 & 0.0 & - & -- \\
\hline & & 25.4 & 26.0 & 8.2 & -37.5 & -36.5 & 56.4 & 13.3 & -- \\
\hline-9.7 & $\begin{array}{r}-4.5 \\
0.0\end{array}$ & $\begin{array}{r}-20.3 \\
0.0\end{array}$ & $\begin{array}{r}-34.8 \\
0.0\end{array}$ & $\begin{array}{r}17.3 \\
0.0\end{array}$ & -26.0 & $\overline{0}$ & $\overline{0}$ & $=-$ & $=$ \\
\hline 56.5 & 57.4 & 58.3 & 55.9 & 5.1 & 51.9 & 51.4 & 59.4 & -- & -- \\
\hline
\end{tabular}

Source: "Balance of Payments Statistics", 1988, IMF. 


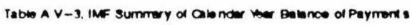

\begin{tabular}{|c|c|c|c|c|c|c|c|c|c|c|c|c|c|c|c|c|c|c|c|c|c|c|c|c|c|c|c|c|c|c|}
\hline & & & & & & & & & & & & & & & & & & & & & & & & & & & & & & \\
\hline & Actuat & 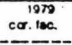 & .4at12 & extuat & 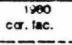 & cot $\|_{2}$ & sctual & caltion. & actitig & Netuat & 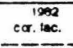 & 001112 & cruat c & 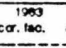 & sotng & Actual : & cotion. & solnge & setuat co & 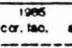 & $\therefore$ & Nectoon & 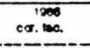 & .0. & Nectuar & cos & $-04 \mathrm{ng}$ & Natuat. & 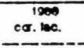 & ․ㅜㅇt \\
\hline Curen nocourn & $-\infty .8$ & & $-\infty .8$ & 324 & & 324 & -2003 & & -20.3 & -2.0 & & -2.0 & -100.4 & & -119.4 & -50.6 & & $-\$ 0.8$ & -209.1 & & -240.1 & -586.3 & & -868.3 & -- & & 0.0 & -- & & 0.0 \\
\hline 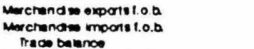 & 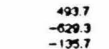 & $\begin{array}{l}10 \infty \\
1 . \infty\end{array}$ & 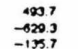 & 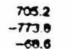 & $\begin{array}{l}10 \\
1.0\end{array}$ & 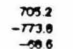 & 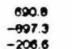 & $\begin{array}{l}100 \\
1 . \infty\end{array}$ & 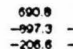 & 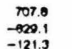 & $\begin{array}{l}1 . \infty \\
1 . \infty\end{array}$ & 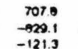 & $\begin{array}{c}r 20.0 \\
\substack{+\infty 00 \\
-10.1}\end{array}$ & $\begin{array}{l}1.0 \\
1.00\end{array}$ & 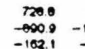 & 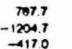 & $1 . \infty-$ & $\begin{array}{l}7077 \\
-12077 \\
-1107\end{array}$ & 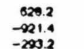 & $\begin{array}{l}100 \\
1.00\end{array}$ & 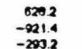 & 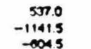 & $\begin{array}{l}1 \infty \\
1 \infty \infty\end{array}$ & 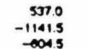 & $\because$ & $\begin{array}{l}100 \\
1.00\end{array}$ & $\begin{array}{l}0.0 \\
0.0 \\
0.0\end{array}$ & $=$ & $\begin{array}{l}1: \infty \\
1 . \infty\end{array}$ & $\begin{array}{l}\because 0 \\
00 \\
0\end{array}$ \\
\hline 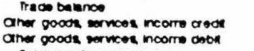 & $\begin{array}{l}-135.1 \\
-1210 \\
-100.1\end{array}$ & $\begin{array}{l}10 \infty \\
1 . \infty\end{array}$ & $\begin{array}{l}-133.7 \\
-120.0 \\
-100.1\end{array}$ & $\begin{array}{l}-60.6 \\
-97.5 \\
-150.7\end{array}$ & $i_{i \infty}^{\infty}$ & 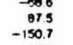 & $\begin{array}{l}-2006 \\
75.5 \\
-1032\end{array}$ & $\begin{array}{l}10 \infty \\
1 . \infty\end{array}$ & 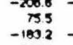 & 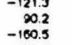 & $\begin{array}{l}1.0 \\
1.0\end{array}$ & $\begin{array}{l}-1213 \\
202 \\
-1005\end{array}$ & $\begin{aligned} &-12.1 \\
&-172 . \\
&-120 .\end{aligned}$ & $\mathfrak{1}_{1, \infty}^{1 \infty}$ & $\left.\begin{array}{c}-128.1 \\
774 \\
-1720\end{array}\right]$ & 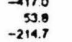 & $\left.\begin{array}{l}10 \\
1.00\end{array}\right]$ & $\begin{array}{l}-5110 \\
-2147 \\
-214\end{array}$ & $\begin{array}{l}-2002 \\
-6022 \\
-162.7\end{array}$ & $\begin{array}{l}100 \\
1.00\end{array}$ & $\begin{array}{l}-2022 \\
6022 \\
-1627\end{array}$ & $\begin{array}{l}-8015 \\
2123 \\
-2155\end{array}$ & $\begin{array}{l}1, \infty \\
1 . \infty\end{array}$ & $\begin{array}{l}-8015 \\
723 \\
-215.5\end{array}$ & $\bar{z}$ & $i_{\substack{1 \\
1 \infty}}$ & $\begin{array}{l}0.0 \\
0.0 \\
0.0\end{array}$ & $\because=$ & $\begin{array}{l}1, \infty \\
1, \infty\end{array}$ & $\begin{array}{l}\because 0 \\
0\end{array}$ \\
\hline 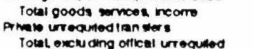 & -101.8 & 1.00 & $\begin{array}{r}-181.8 \\
0.0\end{array}$ & $\begin{array}{l}-1310 \\
14.4\end{array}$ & 1.00 & $\begin{array}{l}-193.8 \\
-130 . \\
194\end{array}$ & 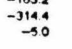 & $1 . \infty$ & $\begin{array}{l}-319.4 \\
-3.0 .0\end{array}$ & $\begin{array}{l}-106.5 \\
-197.7 \\
10.4\end{array}$ & $1 . \infty$ & $\begin{array}{l}-2013 \\
-191.7 \\
18.4\end{array}$ & $\begin{array}{l}-180.7 \\
0.03\end{array}$ & 1.00 & $\begin{array}{l}-2807 \\
0.3\end{array}$ & 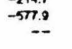 & $1 . \infty$ & $\begin{array}{l}-5770 \\
-570\end{array}$ & -3006 & $1 . \infty$ & -3006 & -7478 & 1.00 & $\begin{array}{l}-2135 \\
-747.8 \\
0.0\end{array}$ & $=$ & 1.00 & $\begin{array}{l}\because 0 \\
0.0 \\
0\end{array}$ & $\because$ & 1.00 & $\begin{array}{l}\because 0 \\
0.0\end{array}$ \\
\hline 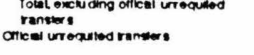 & $\begin{array}{l}-181.9 \\
823\end{array}$ & $\begin{array}{l}1: \infty \\
: \infty \infty\end{array}$ & $\begin{aligned}-189.9 \\
02.3\end{aligned}$ & $\begin{array}{l}-117: 8 \\
149.8\end{array}$ & $\begin{array}{l}1 \infty \\
1 . \infty\end{array}$ & -1174 & $\begin{array}{l}-31922 \\
00.9\end{array}$ & $\begin{array}{l}1: \infty \\
1: \infty\end{array}$ & $\begin{array}{l}-3192.2- \\
0 \infty .0\end{array}$ & $\begin{array}{l}-17522 \\
62.4\end{array}$ & $\begin{array}{l}1 \infty \\
1, \infty\end{array}$ & $\begin{array}{l}-1752 \\
024\end{array}$ & -200.4 & 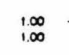 & $\underset{\substack{-200.0 \\
\infty 0.0}}{-1}$ & $\begin{array}{c}-57,9 \\
127.3\end{array}$ & $\underset{1: \infty}{1 \infty}$ & $\begin{array}{l}-57.9 \\
127.3\end{array}$ & $\begin{array}{l}-306.7 \\
10.7\end{array}$ & $\begin{array}{l}1, \infty \\
1 . \infty\end{array}$ & $-\frac{90.7}{160.7}$ & $\begin{array}{r}-747.7 \\
191.3\end{array}$ & $\begin{array}{l}1 \infty \infty \\
1: \infty\end{array}$ & $\begin{array}{c}-747,7 \\
101.3\end{array}$ & $\because$ & $i_{i \infty}^{\infty}$ & $\because 0$ & $\because$ & $\underbrace{100}_{1: \infty}$ & $\because$ \\
\hline 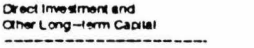 & $m 3$ & & 177.3 & 1503 & & 150.3 & so & & es. 0 & ros & & ros & 210 & & 21.0 & 57.0 & & 570 & $n 6$ & & 7.8 & 28.7 & & 208.7 & -- & & 00 & -- & & 0.0 \\
\hline 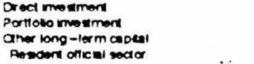 & $\begin{array}{r}00 \\
179 \\
119.9 \\
119.4\end{array}$ & $\begin{array}{l}1 \infty \\
1 \infty \\
1 \infty\end{array}$ & 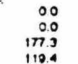 & $\begin{aligned} 0.0 \\
1590 \\
159 . \\
100\end{aligned}$ & $\begin{array}{l}1 \infty \\
1 . \infty \\
1 \infty\end{array}$ & $\begin{array}{r}0.0 \\
1500 \\
150.3 \\
100\end{array}$ & $\begin{array}{l}\therefore 0 \\
\therefore 0 \\
\infty \times 0 \\
\infty\end{array}$ & $\begin{array}{l}1 \infty \\
1 . \infty \\
1 \infty\end{array}$ & 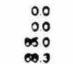 & $\begin{array}{l}0.0 \\
0.0 \\
70.5 \\
05\end{array}$ & $\begin{array}{l}1 \infty \\
1 \infty \\
1 \infty\end{array}$ & $\begin{array}{l}0.0 \\
00 \\
005 \\
015\end{array}$ & $\begin{array}{l}00 \\
0.0 \\
210 \\
07\end{array}$ & $\begin{array}{l}1 \\
1 . \infty \\
1 \infty\end{array}$ & $\begin{array}{l}0.0 \\
0.0 \\
210 \\
07\end{array}$ & $\begin{array}{l}0.0 \\
0.0 \\
570 \\
\rightarrow 0\end{array}$ & $\begin{array}{l}1 \infty \\
1, \infty \\
1 \infty\end{array}$ & $\begin{array}{l}0: 0 \\
050 \\
5 \rightarrow 0\end{array}$ & $\begin{array}{l}00 \\
0.0 \\
076 \\
n i\end{array}$ & $\begin{array}{l}1 \infty \\
1 \infty \\
1 \infty\end{array}$ & $\begin{array}{l}00 \\
0.0 \\
710\end{array}$ & $\begin{aligned} 00 \\
0.0 \\
2507\end{aligned}$ & $\begin{array}{l}100 \\
1.0 \\
1 \infty\end{array}$ & 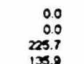 & $=$ & $\begin{array}{l}1 \infty \\
1 \infty \\
1 \infty\end{array}$ & $\begin{array}{l}\because 0 \\
\therefore 0 \\
\therefore 0\end{array}$ & $=$ & $\begin{array}{l}1 . \infty \\
1 . \infty \\
10\end{array}$ & $\begin{array}{l}\because 0 \\
\therefore \\
\therefore 0\end{array}$ \\
\hline 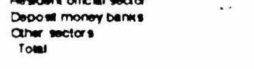 & $\begin{array}{c}0.0 \\
57.0 \\
77.6 \\
.\end{array}$ & $\begin{array}{l}1,0 \\
1, \infty \\
1 . \infty\end{array}$ & $\begin{array}{l}10.4 \\
57.0 \\
7.6\end{array}$ & $\begin{aligned} 110.8 \\
0.0 \\
10.5 \\
10.7\end{aligned}$ & $\begin{array}{l}1 \\
1 \\
1 \\
1 \infty \infty \\
1 \infty\end{array}$ & $\begin{aligned} 10.0 \\
0.0 \\
0.5 \\
191.7\end{aligned}$ & $\begin{aligned} 0.3 \\
0.0 \\
107 \\
-107 \\
-193\end{aligned}$ & $\begin{array}{l}1 \\
1 \\
1 \\
1.0 \\
1.0\end{array}$ & $\begin{array}{rl}0 & 0.3 \\
0.0 \\
16.7 \\
-164.3\end{array}$ & $\begin{array}{r}203 \\
0.0 \\
27.0 \\
-22.3\end{array}$ & $\begin{array}{l}1, \infty \\
100 \\
1 . \infty\end{array}$ & $\begin{aligned} 23.5 \\
200 \\
270 \\
-223\end{aligned}$ & $\begin{aligned} 0.7 \\
0.0 \\
112 \\
-12.5\end{aligned}$ & $\begin{array}{l}1: \infty \\
1 \\
1 . \infty \\
1 . \infty\end{array}$ & $\begin{aligned} 9.7 \\
0.0 \\
-11.2 \\
-120.5\end{aligned}$ & 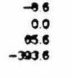 & $\begin{array}{l}1 \\
1 \\
1 \\
1 \\
1 \infty \\
\infty\end{array}$ & 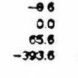 & $\begin{aligned} 20.2 \\
0.0 \\
0.4 \\
-105.5\end{aligned}$ & $\begin{array}{l}1.00 \\
100 \\
1.00\end{array}$ & $\begin{aligned} 222 \\
00 \\
5.4 \\
-150.5\end{aligned}$ & 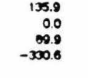 & $\begin{array}{l}1: \infty \\
1: \infty \\
1 . \infty\end{array}$ & $\begin{array}{r}135.9 \\
000 \\
-300.8\end{array}$ & $\begin{array}{l}\because \\
\because \\
z\end{array}$ & $\begin{array}{l}1, \infty \\
10 \\
1.0 \\
1 . \infty\end{array}$ & $\begin{array}{l}00 \\
\therefore 0 \\
0.0 \\
0.0\end{array}$ & $\begin{array}{l}\because \\
\because\end{array}$ & $\begin{array}{l}100 \\
100 \\
1.00\end{array}$ & $\begin{array}{l}\because 0 \\
\therefore \\
0.0\end{array}$ \\
\hline 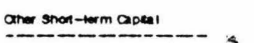 & -61.0 & & -81.9 & -20.6 & & -284.6 & -100 & & -100 & $\infty .0$ & & $\infty 8.0$ & 100.1 & & 100.1 & 230.3 & & 280.3 & -- & & 0.0 & -- & & 0.0 & -- & & 0.0 & -- & & 0.0 \\
\hline 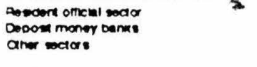 & $\begin{array}{l}-112 \\
-0.8 \\
--\end{array}$ & $\begin{array}{l}10 \\
100 \\
1 . \infty \\
1 . \infty\end{array}$ & $\begin{array}{l}-812 \\
-0.8 \\
00\end{array}$ & $\begin{array}{c}-x+0.0 \\
-0.7 \\
--\end{array}$ & $\begin{array}{l}1, \infty \\
1,0 \\
1, \infty\end{array}$ & $\begin{array}{c}-x+20,0 \\
-0,7 \\
0.0\end{array}$ & $\begin{array}{l}-20.1 \\
11.1 \\
-1\end{array}$ & $\begin{array}{l}100 \\
1 \infty \\
1 . \infty\end{array}$ & $\begin{array}{c}-x .1 \\
1.1 \\
0.0\end{array}$ & $\begin{array}{c}n .1 \\
-7.1 \\
--\end{array}$ & $\begin{array}{l}1, \infty \\
10 \infty \\
1 . \infty\end{array}$ & $\begin{array}{c}n \rightarrow 1 \\
\substack{n \rightarrow 1 \\
0.0}\end{array}$ & $\begin{array}{l}1400 \\
1306 \\
--\end{array}$ & $\begin{array}{l}1 \\
1 \infty \\
1 \infty \infty \\
1 \infty\end{array}$ & $\begin{array}{c}1408 \\
136 \\
0.0\end{array}$ & $\begin{array}{r}x 1.5 \\
:-7\end{array}$ & $\begin{array}{l}1, \infty \\
10 \infty \\
1 . \infty\end{array}$ & $\begin{array}{r}251.5 \\
17 \\
00\end{array}$ & $\because:$ & $\begin{array}{l}10 \\
1, \infty \\
10 \infty\end{array}$ & $\begin{array}{l}00 \\
0.0 \\
0.0\end{array}$ & $=$ & $\begin{array}{l}1: \infty \\
1,0 \\
10 \infty\end{array}$ & $\begin{array}{l}\because 0 \\
\therefore\end{array}$ & $\because=$ & $\begin{array}{l}1 \\
1 \\
1 \\
1 \infty \\
\infty\end{array}$ & $\begin{array}{l}\because 0 \\
\therefore 0 \\
\therefore 0\end{array}$ & $\because$ & $\begin{array}{l}1 \infty \\
1 \infty \\
1 \infty \\
1 \infty\end{array}$ & $\begin{array}{l}0.0 \\
\therefore .0 \\
0.0\end{array}$ \\
\hline 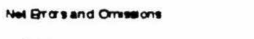 & -205 & 1.00 & -2.5 & 12 & 100 & 12 & 1243 & 10 & 1245 & -31.7 & 1.00 & -51.7 & $-\infty+4$ & $1 . \infty$ & $-\infty 4$ & 2077 & 1.00 & 2017 & 204.4 & 100 & 2344 & 203 & 1.00 & 2003 & -- & 1.00 & $\infty 0$ & -- & 100 & $\infty$ \\
\hline row & $\rightarrow$ & & $\rightarrow 0$ & י.יר & & -7 & -379 & & -7.0 & -79 & & -79 & -50.0 & & -500 & nos & & 20.3 & soo & & so.9 & $-m, 3$ & & $-\pi, 3$ & -- & & 00 & -- & & 0.0 \\
\hline 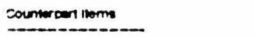 & 14 & & 1.4 & 10.8 & & 108 & $\infty$ & & ж.0 & 189 & & 109 & 133 & & 15.5 & -1.5 & &.- .5 & -2.9 & & -229 & -30.0 & & -300 & -- & & 0.0 & -- & & 0.0 \\
\hline 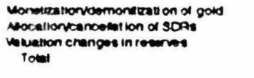 & 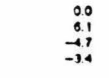 & $\begin{array}{l}1 . \infty \\
10 \infty \\
1 . \infty\end{array}$ & $\begin{array}{l}0.0 \\
\therefore: 1 \\
-3.1\end{array}$ & $\begin{array}{l}0.0 \\
0.1 \\
\text { a.1. } \\
-53.1\end{array}$ & $\begin{array}{l}100 \\
100 \\
10 \infty\end{array}$ & 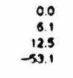 & 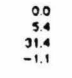 & $\begin{array}{l}1 \\
100 \\
10 \infty \\
1 \infty\end{array}$ & 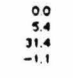 & $\begin{array}{l}\therefore 0 \\
\therefore 0 \\
16.9 \\
0.9\end{array}$ & $\begin{array}{l}1 \\
100 \\
1 . \infty \\
1 . \infty\end{array}$ & $\begin{array}{l}\therefore 0 \\
\therefore 0 \\
10.0 \\
0.0\end{array}$ & $\begin{array}{l}0.0 \\
159 \\
159 \\
-40\end{array}$ & $\begin{array}{l}1 \\
1 \infty \\
1 \infty \\
1 \infty\end{array}$ & $\begin{aligned} 0.0 \\
0.0 \\
15.5 \\
-\rightarrow 3\end{aligned}$ & $\begin{array}{l}\therefore 0 \\
-15 \\
-i s \\
\infty\end{array}$ & $\begin{array}{l}1 \\
100 \\
1 . \infty\end{array}$ & $\begin{array}{l}00 \\
\therefore 0 \\
-i s \\
-\infty .0\end{array}$ & $\begin{aligned} \therefore 0 \\
200 \\
-20.0 \\
350\end{aligned}$ & $\begin{array}{l}1 \\
\vdots \\
i \infty \\
1 \infty \\
\infty\end{array}$ & $\begin{aligned} 00 \\
200 \\
-20.9 \\
3.9\end{aligned}$ & $\begin{aligned} 0.0 \\
0.0 \\
-300 \\
-67.3\end{aligned}$ & $\begin{array}{l}1 \infty \\
1 \\
1, \infty \\
1 . \infty\end{array}$ & $\begin{aligned} 00 \\
-00 \\
-300 \\
-8,0\end{aligned}$ & $\begin{array}{l}z \\
\because=\end{array}$ & $\begin{array}{l}1: \infty \\
\vdots \infty \\
1 \infty \infty\end{array}$ & $\begin{array}{l}0.0 \\
0 . \\
0 . \\
0\end{array}$ & $\begin{array}{l}z \\
\because=\end{array}$ & $\begin{array}{l}1: \infty \\
1: \infty \\
1 . \infty\end{array}$ & $\begin{array}{l}\because 0 \\
\therefore 0 \\
0.0\end{array}$ \\
\hline 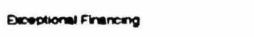 & 0.0 & $1 . \infty$ & $\infty .0$ & 0.0 & 1.00 & 0.0 & 0.0 & 1.00 & 0.0 & 0.0 & 1.00 & 0.0 & 0.0 & $1 . \infty$ & 0.0 & 0.0 & 1.00 & 0.0 & 0.0 & 1.00 & 0.0 & 0.0 & 1.00 & 0.0 & -- & 1.00 & 0.0 & -- & 1.00 & 0.0 \\
\hline Toues & -34 & & -3.4 & -301 & & -53.1 & $-י$ & & $-1 .$, & 0.9 & & 0.9 & $-4 s$ & & -43 & $\infty$ & & $\infty$ & 3.9 & & 35.9 & -87.3 & & -8.3 & -- & & 0.0 & -- & & 0.0 \\
\hline 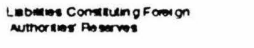 & 00 & $1 . \infty$ & 00 & 00 & 1.00 & 0.0 & 0.0 & 1.00 & 0.0 & 0.0 & 1.00 & $\infty 0$ & 0.0 & $1 \infty$ & 0.0 & 0.0 & 1.00 & 0.0 & 0.0 & 100 & 0.0 & 0.0 & 1.00 & 0.0 & -- & 1.00 & 0.0 & -- & 1.00 & 0.0 \\
\hline rom & -34 & & -3. & -30.1 & & -3.1 & -1.1 & & $-1 .$, & .9 & & 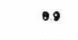 & $-a$ & & נו & $\infty$ & & $\infty$ & 3.9. & & s. & (7.3 & & -87.3 & -- & & 0.0 & -- & & 0.0 \\
\hline 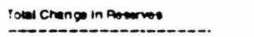 & 3.4 & & 3. & 53.1 & & 50.1 & .1 & & 1.1 & $\rightarrow .9$ & & $\rightarrow \rightarrow$ & as & & 43 & $-\infty .7$ & & $-\infty .7$ & -35.0 & & -3.9 & 67.3 & & 67.3 & 10.4 & & 18.4 & 1.3 & & 1.3 \\
\hline 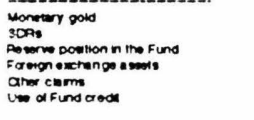 & $\begin{array}{r}00 \\
-10.3 \\
-0.0 \\
28.0 \\
-125 \\
0.0\end{array}$ & 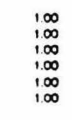 & $\begin{aligned} 0.0 \\
-103 \\
-0.0 \\
-08 \\
-125 \\
-125 \\
0.0\end{aligned}$ & $\begin{array}{r}-0.1 \\
1.7 \\
-7.3 \\
6.7 \\
-5.9 \\
0.0\end{array}$ & $\begin{array}{l}100 \\
100 \\
100 \\
100 \\
10 \infty \\
1, \infty\end{array}$ & $\begin{array}{l}-0.1 \\
.17 \\
-7, \\
-6,7 \\
-39 \\
0.0\end{array}$ & $\begin{array}{r}0.0 \\
-50 \\
0.0 \\
000 \\
-230 \\
0.0\end{array}$ & 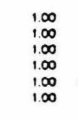 & $\begin{array}{r}0.0 \\
-30 \\
00 \\
000 \\
-200 \\
0.0 \\
0.0\end{array}$ & $\begin{aligned} 0.0 \\
0.0 \\
0.0 \\
0.07 \\
-0.4 \\
0.0\end{aligned}$ & 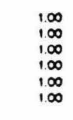 & $\begin{array}{r}00 \\
\therefore 0 \\
0.0 \\
20.7 \\
-30.4 \\
0.0\end{array}$ & $\begin{array}{l}80 \\
80 \\
110 \\
\therefore 0.0 \\
10.5 \\
0.0\end{array}$ & $\begin{array}{l}1, \infty \\
1, \infty \\
1, \infty \\
1 \infty \\
1 \infty \\
1 . \infty \\
1 . \infty\end{array}$ & $\begin{array}{l}00 \\
180 \\
110 \\
105 \\
105 \\
0.0\end{array}$ & $\begin{array}{r}0.0 \\
-3.6 \\
-0.0 \\
-0.04 \\
-607 \\
0.0\end{array}$ & $\begin{array}{l}1 \\
1 \\
1 \\
1 \\
1 \\
1 \infty \\
1 \\
1 \infty \\
1 \infty\end{array}$ & $\begin{array}{l}00 \\
-3.8 \\
0.0 \\
-30.4 \\
-20.7 \\
0.0\end{array}$ & $\begin{array}{r}0.0 \\
11 \\
00.0 \\
-37.1 \\
0.0\end{array}$ & $\begin{array}{l}1 \infty \\
10 \infty \\
1 \infty \\
1 \infty \\
1 \infty \\
1 \infty \infty \\
1 . \infty\end{array}$ & $\begin{array}{r}0.0 \\
0.1 \\
070 . \\
0.0 \\
0.0\end{array}$ & $\begin{array}{l}0.0 \\
1.2 \\
0.0 \\
06.2 \\
-0.0 \\
0.0\end{array}$ & 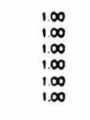 & $\begin{array}{l}0.0 \\
0.2 \\
1.2 \\
0 \infty 0 \\
0.2 \\
0.0 \\
0.0\end{array}$ & $\begin{array}{l}7-2 \\
12 \\
172 \\
--\end{array}$ & 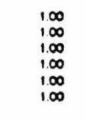 & $\begin{array}{l}0.0 \\
12 \\
1.2 \\
0.0 \\
172 \\
0.0 \\
0.0\end{array}$ & $\begin{array}{l}-3 \\
=- \\
=- \\
=\end{array}$ & $\begin{array}{l}1 \\
10 \\
10 \\
100 \\
100 \\
10 \infty \\
1 . \infty\end{array}$ & $\begin{array}{l}0.0 \\
i g \\
\therefore 0 \\
\therefore 0 \\
\therefore 0 \\
0.0\end{array}$ \\
\hline comementrabe Algrem ow U.SS & מבת & & & 4.10 & & & s.e. & & & 5000 & & & 50.81 & & & 50.83 & & & 50.62 & & & 50.00 & & & -- & & & -- & & \\
\hline
\end{tabular}




\begin{tabular}{|c|c|c|c|c|c|c|c|c|c|}
\hline 1979 & 1980 & 1981 & 1982 & 1983 & 1984 & 1985 & 1986 & 1987 & 1988 \\
\hline-77.1 & 24.9 & -194.5 & -84.1 & -139.8 & -439.6 & -239.4 & -474.2 & 0.0 & 0.0 \\
\hline 382.1 & 541.8 & 585.8 & 641.1 & 681.8 & 768.5 & 618.7 & 457.7 & -- & -- \\
\hline-487.1 & -594.5 & -761.0 & -751.0 & -833.4 & -1175.3 & -907.5 & -973.0 & -- & -- \\
\hline-105.0 & -52.7 & -175.2 & -109.9 & -151.6 & -406.8 & -288.8 & -515.3 & 0.0 & 0.0 \\
\hline 94.3 & 67.2 & 64.0 & 81.7 & 72.4 & 52.5 & 68.2 & 61.6 & -- & -- \\
\hline-130.1 & -115.8 & -155.4 & -145.4 & -160.9 & -209.5 & -160.2 & -183.7 & -- & -- \\
\hline-140.8 & -101.3 & -266.6 & -173.6 & -240.1 & -563.8 & -380.8 & -637.4 & 0.0 & 0.0 \\
\hline-- & 11.1 & -4.2 & 14.9 & 7.8 & -- & -- & -- & -- & -- \\
\hline-140.8 & -90.2 & -270.7 & -158.7 & -232.4 & -563.8 & -380.9 & -637.3 & -- & -- \\
\hline 63.7 & 115.1 & 76.2 & 74.6 & 92.6 & 124.2 & 141.5 & 163.1 & -- & -- \\
\hline 137.2 & 122.4 & 72.1 & 63.9 & 19.6 & 55.6 & 76.4 & 192.4 & 0.0 & 0.0 \\
\hline 0.0 & 0.0 & 0.0 & 0.0 & 0.0 & 0.0 & 0.0 & 0.0 & -- & -- \\
\hline 0.0 & 0.0 & 0.0 & 0.0 & 0.0 & 0.0 & 0.0 & 0.0 & -- & -- \\
\hline 137.2 & 122.4 & 72.1 & 63.9 & 19.6 & 55.6 & 76.4 & 192.4 & 0.0 & 0.0 \\
\hline 92.4 & 85.1 & 57.9 & 39.4 & 9.1 & -8.4 & 31.7 & 115.8 & -- & -- \\
\hline 0.0 & 0.0 & 0.0 & 0.0 & 0.0 & 0.0 & 0.0 & 0.0 & -- & -- \\
\hline 44.8 & 37.3 & 14.2 & 24.5 & 10.5 & 64.0 & 44.7 & 76.6 & -- & -- \\
\hline 60.1 & 147.3 & -122.4 & -20.2 & -120.2 & -384.0 & -163.0 & -281.8 & 0.0 & 0.0 \\
\hline-47.9 & -203.3 & -15.3 & 59.8 & 152.6 & 250.0 & -- & -- & 0.0 & 0.0 \\
\hline-47.4 & -202.8 & -24.7 & 66.2 & 139.9 & 245.4 & -- & -- & -- & -- \\
\hline-0.5 & -0.5 & 9.4 & -6.4 & 12.7 & 4.6 & -- & -- & -- & -- \\
\hline-- & -- & -- & -- & -- & -- & -- & -- & -- & -- \\
\hline-15.9 & 0.9 & 105.6 & -46.8 & -88.3 & 202.6 & 221.0 & 250.0 & -- & -- \\
\hline-3.7 & -55.1 & -32.1 & -7.2 & -55.9 & 68.6 & 58.0 & -31.8 & 0.0 & 0.0 \\
\hline 1.1 & 14.3 & 31.2 & 15.3 & 14.5 & -1.5 & -22.6 & -25.6 & 0.0 & 0.0 \\
\hline 0.0 & 0.0 & 0.0 & 0.0 & 0.0 & 0.0 & 0.0 & 0.0 & -- & -- \\
\hline 4.7 & 4.7 & 4.6 & 0.0 & 0.0 & 0.0 & 0.0 & 0.0 & -- & -- \\
\hline-3.6 & 9.6 & 26.6 & 15.3 & 14.5 & -1.5 & -22.6 & -25.6 & -- & -- \\
\hline-2.6 & -40.8 & -0.9 & 8.1 & -41.4 & 67.1 & 35.4 & -57.4 & 0.0 & 0.0 \\
\hline 0.0 & 0.0 & 0.0 & 0.0 & 0.0 & 0.0 & 0.0 & 0.0 & -- & -- \\
\hline-2.6 & -40.8 & -0.9 & 8.1 & -41.4 & 67.1 & 35.4 & -57.4 & 0.0 & 0.0 \\
\hline 0.0 & 0.0 & 0.0 & 0.0 & 0.0 & 0.0 & 0.0 & 0.0 & -- & -- \\
\hline-2.6 & -40.8 & -0.9 & 8.1 & -41.4 & 67.1 & 35.4 & -57.4 & 0.0 & 0.0 \\
\hline 2.6 & 40.8 & 0.9 & -8.1 & 41.4 & -67.0 & -35.4 & 57.4 & 14.2 & 1.0 \\
\hline 0.0 & -0.1 & 0.0 & 0.0 & 0.0 & 0.0 & 0.0 & 0.0 & -- & -- \\
\hline-8.0 & 1.3 & -4.2 & 0.7 & 5.6 & -3.5 & 1.1 & 1.0 & 0.9 & 1.0 \\
\hline-0.5 & -5.6 & 0.0 & 0.0 & 10.3 & 0.0 & 0.0 & 0.0 & -- & -- \\
\hline 20.8 & 49.7 & 25.4 & 26.0 & 8.2 & -37.5 & -36.5 & 56.4 & 13.3 & -- \\
\hline-9.7 & -4.5 & -20.3 & -34.8 & 17.3 & -26.0 & -- & -- & -- & -- \\
\hline 0.0 & 0.0 & 0.0 & 0.0 & 0.0 & 0.0 & 0.0 & 0.0 & -- & -- \\
\hline 6.5 & 57.4 & ! & J & ;4.1 & 51. & & J & -- & -1 \\
\hline
\end{tabular}


Table A V-4. IMF Summary of Bilateral Balance of Payments

(millions of U.S. dollars)

\begin{tabular}{|c|c|c|c|c|c|}
\hline & $1981 / 82$ & $1982 / 83$ & $1983 / 84$ & $1984 / 85$ & $1985 / 86$ \\
\hline Trade balance & -124.1 & -267.6 & -300.4 & -419.6 & -255.6 \\
\hline Exports, f.o.b. & $617.2[\mathrm{a}]$ & 412.6 & 311.5 & 412.9 & 463.8 \\
\hline Imports, c.i.f. & -741.3 & -680.2 & -611.9 & -832.5 & -719.4 \\
\hline Commercial & -531.3 & -440.9 & -418.0 & -558.1 & -432.3 \\
\hline Project aid [b] & -122.2 & -156.8 & -131.7 & -150.1 & -141.3 \\
\hline Commodity aid & -87.8 & -82.5 & -62.2 & -124.3 & -145.8 \\
\hline Services & 1.3 & 50.0 & 33.9 & 9.5 & 23.6 \\
\hline Receipts & 42.3 & 72.4 & 53.4 & 29.0 & 32.2 \\
\hline Payments, of which & -41.0 & -22.4 & -19.5 & -19.5 & -8.6 \\
\hline Investment income [c] & -11.8 & -13.3 & -13.6 & -13.3 & -1.8 \\
\hline Current Account & -122.8 & -217.6 & -266.5 & -410.1 & -232.0 \\
\hline Nonmonetary capital & 151.9 & 144.5 & 117.0 & 182.0 & 236.9 \\
\hline Receipts & 232.8 & 243.2 & 199.8 & 276.0 & 288.6 \\
\hline Public loans & 137.8 & 149.2 & 101.3 & 91.1 & 112.0 \\
\hline Suppliers' credits & 16.7 & 27.0 & 11.2 & 65.6 & 45.4 \\
\hline Grants [d] & 78.3 & 67.0 & 87.3 & 119.3 & 131.2 \\
\hline Repayments & -80.9 & -98.7 & -82.8 & -94.0 & -51.7 \\
\hline Errors and ommissions, net & -- & -- & -- & $-23.4[\mathrm{e}]$ & $-29.5[e]$ \\
\hline Overall balance & 29.1 & -73.1 & -149.5 & -251.5 & -24.6 \\
\hline Monetary movements & -29.1 & 73.1 & 149.5 & 251.5 & 24.6 \\
\hline Central bank & -29.1 & 73.1 & 149.5 & 251.5 & 24.6 \\
\hline Commercial banks & -- & -- & -- & -- & -- \\
\hline
\end{tabular}

[a] Adjusted for underestimation of receipts in the customs estimates as a net positive errors and ommissions entry in the bilateral balance of payments account.

[b] Includes imports financed by suppliers' credits and public loans.

[c] Includes interest on foreign debt.

[d] Mostly counterpart of noncash grants.

[e] Represents increases in bilateral debit balances with the USSR that were made available in convertible currencies. 
Table A V - 5. IMF Summary of Multilateral Balance of Payments

(millions of U.S. dollars)

\begin{tabular}{|c|c|c|c|c|c|}
\hline & $1981 / 82$ & $1982 / 83$ & $1983 / 84$ & $1984 / 85$ & $1985 / 86$ \\
\hline Trade balance & -24.1 & 37.5 & 4.0 & -248.0 & -175.5 \\
\hline Exports, f.o.b. & 265.9 & 331.2 & 317.2 & 234.9 & 164.4 \\
\hline Imports, c.i.f. & -290.0 & -293.7 & -313.2 & -482.9 & -339.9 \\
\hline Commercial & -264.9 & -275.2 & -299.2 & -470.4 & -321.3 \\
\hline Project aid [a] & -24.0 & -17.4 & -13.6 & -11.9 & -18.6 \\
\hline Commodity aid & -1.1 & -1.1 & -0.4 & -0.6 & -- \\
\hline Services & 20.0 & 20.0 & 12.9 & 16.4 & 20.6 \\
\hline Receipts & 56.7 & 43.3 & 34.6 & 31.7 & 37.0 \\
\hline Payments, of which & -36.7 & -23.3 & -21.7 & -15.3 & -16.4 \\
\hline Investment income [b] & -3.5 & -6.8 & -7.8 & -5.2 & -6.8 \\
\hline Current Account & -4.1 & 57.5 & 16.9 & -231.6 & -154.9 \\
\hline Nonmonetary capital & 23.0 & 8.4 & 2.9 & 2.3 & -15.6 \\
\hline Receipts & 39.9 & 23.4 & 18.5 & 15.8 & 22.5 \\
\hline Public loans & 28.3 & 8.0 & 6.8 & 7.8 & 10.0 \\
\hline Suppliers' credits & -- & -- & -- & -- & -- \\
\hline Grants [c] & 11.6 & 15.4 & 11.7 & 8.0 & 12.5 \\
\hline Repayments & -16.9 & -15.0 & -15.6 & -13.5 & -38.1 \\
\hline Errors and ommissions, net & -80.9 & -63.1 & -85.6 & 302.2 & 234.0 \\
\hline Overall balance & -62.0 & 2.8 & -65.8 & 72.9 & 63.5 \\
\hline Monetary movements & 62.0 & -2.8 & 65.8 & -72.9 & -63.5 \\
\hline Central bank & 69.5 & 38.7 & 28.4 & -40.4 & -62.1 \\
\hline Commercial banks & -7.5 & -41.5 & 37.4 & -32.5 & -1.4 \\
\hline
\end{tabular}

[a] Includes imports financed by suppliers' credits and public loans.

[b] Includes interest on foreign debt.

[c] Mostly counterparts of technical assistance and other services

provided by U.N. organizations such as the UNDP, UNESCO, and WHO.

Source: GOA Da Afghanistan Bank; cited from Afghanistan: Recent Economic

Del me: MF, j. 
Table A V -6. World Bank Summary of Balance of Payments Breakdown

(millions of U.S. dollars)

\begin{tabular}{|c|c|c|c|c|c|c|c|c|}
\hline & $1969 / 70$ & $1970 / 71$ & $1971 / 72$ & $1972 / 73$ & $1973 / 74$ & $1974 / 75$ & $1975 / 76$ & $1976 / 77$ \\
\hline Merchandise trade & -43.1 & -25.7 & -53.5 & -32.6 & -23.8 & -11.9 & -42.0 & -33.4 \\
\hline Exports, fob & 81.7 & 85.1 & 99.7 & 124.5 & 159.1 & 230.6 & 235.5 & 310.0 \\
\hline Imports, cif & -124.8 & -110.8 & -153.2 & -157.1 & -182.9 & -242.5 & -277.5 & -343.4 \\
\hline $\begin{array}{l}\text { Commercial imports } \\
\text { Imports financed by }\end{array}$ & -73.8 & -75.2 & -89.6 & -99.6 & -120.8 & -212.4 & -225.8 & -277.3 \\
\hline $\begin{array}{l}\text { Imports financed by } \\
\text { loans \& grants }\end{array}$ & -51.0 & -35.6 & -63.6 & -57.5 & -62.1 & -30.1 & -51.7 & -66.1 \\
\hline $\begin{array}{l}\text { Services, Transfers and non- } \\
\text { monetary capital }\end{array}$ & 30.6 & 17.6 & 40.2 & 56.0 & 38.1 & 24.9 & 81.3 & 88.1 \\
\hline Travel (net) & 2.7 & 5.2 & 6.3 & 7.0 & 6.8 & 9.0 & 9.0 & 11.0 \\
\hline Service component of Project aid (net) & -3.2 & -5.5 & -4.1 & -4.4 & -6.2 & -12.5 & -16.2 & -18.0 \\
\hline Official loans \& grants & 52.2 & 41.1 & 65.2 & 77.2 & 69.6 & 67.4 & 111.6 & 129.5 \\
\hline Project loans \& grant & 23.6 & 27.6 & 30.7 & 44.2 & 37.2 & 56.0 & 73.1 & 102.0 \\
\hline Non-project loans \& grants & 28.6 & 13.5 & 34.5 & 33.0 & 32.4 & 11.4 & 38.5 & 27.5 \\
\hline External public debt service & -21.1 & -23.2 & -27.2 & -23.8 & -32.1 & -39.0 & -23.1 & -26.9 \\
\hline Principal & -12.6 & -14.2 & -17.8 & -15.6 & -22.5 & -28.5 & -17.9 & -17.7 \\
\hline Interest & -8.5 & -9.0 & -9.4 & -8.2 & -9.6 & -10.5 & -5.2 & -9.2 \\
\hline IBRD Bond purchase & 0.0 & 0.0 & 0.0 & 0.0 & 0.0 & 0.0 & 0.0 & -7.5 \\
\hline $\begin{array}{l}\text { Net residual transactions } \\
\text { (including errors \& omissions) }\end{array}$ & 12.2 & 8.1 & 28.0 & -21.2 & -0.5 & 12.3 & 13.5 & 13.2 \\
\hline Increase in reserves $(-)$ & 0.2 & 0.0 & -14.7 & -2.2 & -13.8 & -25.3 & -52.8 & -67.9 \\
\hline
\end{tabular}

Sources: GOA Da Afghanistan Bank Ministry of Finance, Ministry of Planning and Central Statistical Office; cited from "Afghanistan: The Journey to Economic Development", Vol. II, March 1978, World Bank. 
Table A V-7. External Public and Publicly Guaranteed Debt and Service Payments

(millions of U.S. dollars)

\begin{tabular}{|c|c|c|c|c|c|c|c|c|c|c|c|}
\hline \multirow[b]{2}{*}{ Variable } & \multicolumn{2}{|c|}{$\begin{array}{l}\text { Debt Outstanding } \\
\text { as of March 20,1986 }\end{array}$} & \multirow{2}{*}{$\begin{array}{r}-------- \\
1981 / 82\end{array}$} & \multirow{2}{*}{$\begin{array}{l}- \text {-Debt se } \\
1982 / 83\end{array}$} & \multirow{2}{*}{$\begin{array}{c}\text { rvice paym } \\
1983 / 84\end{array}$} & \multirow{2}{*}{$\begin{array}{l}\text { nts }---- \\
1984 / 85\end{array}$} & \multirow{2}{*}{$1985 / 86$} & \multirow{2}{*}{$\begin{array}{l}---D e b t \\
1986 / 87\end{array}$} & \multirow{2}{*}{$\begin{array}{l}\text { Pervice Proje } \\
1987 / 88\end{array}$} & \multirow{2}{*}{$\begin{array}{r}\text { tions [a] } \\
1988 / 89\end{array}$} & \multirow{2}{*}{$1989 / 90$} \\
\hline & Disbursed & Undisbursed & & & & & & & & & \\
\hline USSR & 2098.4 & 530.5 & 86.2 & 102.8 & 85.0 & 93.8 & 40.2 & 49.0 & 52.0 & 56.5 & 61.0 \\
\hline Principal & -- & -- & 76.3 & 89.5 & 75.5 & 82.6 & 40.2 & 49.0 & 50.1 & 54.4 & 58.5 \\
\hline Interest & -- & -- & 9.9 & 13.3 & 9.5 & 11.2 & -- & -- & 1.9 & 2.1 & 2.5 \\
\hline Czechoslovakia & 117.3 & 32.7 & 6.5 & 9.1 & 11.4 & 13.5 & 13.3 & 11.0 & 13.0 & 13.5 & 14.0 \\
\hline Principal & -- & -- & 4.6 & 9.1 & 7.3 & 11.4 & 11.5 & 8.9 & 10.5 & 11.2 & 11.5 \\
\hline Interest & -- & -- & 1.9 & -- & 4.1 & 2.1 & 1.8 & 2.1 & 2.5 & 2.3 & 2.5 \\
\hline United States & 113.1 & 4.1 & 4.6 & 2.9 & 5.9 & 3.6 & 5.6 & 4.7 & 6.7 & 6.7 & 6.8 \\
\hline Principal & -- & -- & 2.2 & 1.4 & 2.8 & 1.9 & 3.0 & 2.5 & 4.1 & 4.1 & 4.1 \\
\hline Interest & -- & -- & 2.4 & 1.5 & 3.1 & 1.7 & 2.6 & 2.2 & 2.6 & 2.6 & 2.7 \\
\hline German, Fed. Rep. & 100.0 & 7.1 & 5.3 & 5.3 & 4.8 & 3.1 & 3.6 & 4.7 & 4.5 & 4.5 & 4.5 \\
\hline Principal & -- & -- & 4.3 & 4.3 & 4.0 & 2.6 & 3.0 & 3.9 & 3.9 & 3.9 & 3.9 \\
\hline Interest & -- & -- & 1.0 & 1.0 & 0.8 & 0.5 & 0.6 & 0.8 & 0.6 & 0.6 & 0.6 \\
\hline Saudi Arabia & 23.2 & 31.8 & -- & -- & -- & -- & -- & -- & -- & -- & -- \\
\hline Principal & -- & -- & -- & -- & -- & -- & -- & -- & -- & -- & -- \\
\hline Interest & -- & -- & -- & -- & -- & -- & -- & -- & -- & -- & -- \\
\hline Other & 291.3 & 158.4 & 15.3 & 13.7 & 12.6 & 12.0 & 13.0 & 16.6 & 14.7 & 12.0 & 9.5 \\
\hline Principal & -- & -- & 10.4 & 9.4 & 8.7 & 9.0 & 9.4 & 11.9 & 10.9 & 8.1 & 6.6 \\
\hline Interest & -- & -- & 4.9 & 4.3 & 3.9 & 3.0 & 3.6 & 4.7 & 3.8 & 3.9 & 2.9 \\
\hline Total & 2743.3 & 764.6 & 117.9 & 133.8 & 119.7 & 126.0 & 75.7 & 86.0 & 90.9 & 93.2 & 95.8 \\
\hline Principal & -- & -- & 97.8 & 113.7 & 98.3 & 107.5 & 67.1 & 76.2 & 79.5 & 81.7 & 84.6 \\
\hline Interest & -- & -- & 20.1 & 20.1 & 21.4 & 18.5 & 8.6 & 9.8 & 11.4 & 11.5 & 11.2 \\
\hline
\end{tabular}

[a] Based on existing commitments.

Source: GOA Da Afghanistan Bank; cited from Afghanistan: Recent Economic Developments, IMF, 1986. 
Table A V-8. Service Payments, Commitments, Disbursements and Outstanding Amounts of External Public Debt

Projections Based on Debt Outstanding Including Undisbursed as of March 31, 1977

Debt Repayable in Foreign Currency and Goods

TOTAL DEBT OUTSTANDING

(thousands of U.S. dollars)

\begin{tabular}{|c|c|c|c|c|c|c|c|c|c|}
\hline \multirow[b]{3}{*}{ Fiscal Year } & \multicolumn{2}{|c|}{$\begin{array}{l}\text { Debt Outstanding at } \\
\text { Beginning of Period }\end{array}$} & \multicolumn{2}{|c|}{$\begin{array}{l}\text { Total } \\
\text { Transactions During Period }\end{array}$} & \multirow{2}{*}{\multicolumn{2}{|c|}{ Service Payments }} & \multicolumn{3}{|c|}{ Other Changes } \\
\hline & \multirow[b]{2}{*}{$\begin{array}{l}\text { Disbursed } \\
\text { Only }\end{array}$} & \multirow{2}{*}{$\begin{array}{l}\text { Including } \\
\text { Undisbursed }\end{array}$} & \multirow[b]{2}{*}{ Commitments } & \multirow[b]{2}{*}{ Disbursements } & & & \multirow{2}{*}{\multicolumn{2}{|c|}{ Cancellations }} & \multirow{2}{*}{ Adjustments [a] } \\
\hline & & & & & Principal & Interest & & & \\
\hline $1972 / 73$ & 599,649 & 736,599 & 158,043 & 45,738 & 15,215 & 8,228 & 23,443 & 21,246 & 83,624 \\
\hline $1973 / 74$ & 679,547 & 941,805 & 63,006 & 59,585 & 22,618 & 9,579 & 32,197 & 314 & $(8,509)$ \\
\hline $1974 / 75$ & 711,881 & 973,370 & 593,194 & 43,368 & 26,618 & 10,507 & 37,125 & 1,136 & 82,524 \\
\hline $1975 / 76$ & 769,294 & $1,621,334$ & 72,804 & 92,873 & 17,962 & 5,192 & 23,154 & 2,581 & $(83,514)$ \\
\hline $1976 / 77$ & 802,336 & $1,590,081$ & 163,125 & 119,435 & 17,659 & 9,234 & 26,893 & 8 & 12,991 \\
\hline $1977 / 78$ & 911,188 & $1,748,530$ & & & & & & & \\
\hline \multicolumn{2}{|c|}{ 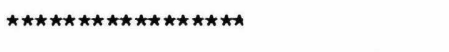 } & \multicolumn{2}{|c|}{ The following figures are projected } & \multicolumn{3}{|c|}{ 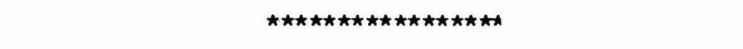 } & & & \\
\hline $1977 / 78$ & 911,188 & $1,748,530$ & 0 & 95,484 & 23,676 & 12,478 & 36,154 & 0 & $(299)$ \\
\hline $1978 / 79$ & 982,697 & $1,724,555$ & 0 & 102,244 & 37,110 & 14,285 & 51,395 & 0 & \\
\hline $1979 / 80$ & $1,047,836$ & $1,687,446$ & 0 & 101,396 & 41,233 & 15,496 & 56,729 & 0 & \\
\hline $1980 / 81$ & $1,107,998$ & $1,646,213$ & 0 & 94,585 & 42,897 & 16,638 & 59,535 & 0 & \\
\hline $1981 / 82$ & $1,159,688$ & $1,603,314$ & 0 & 91,194 & 61,300 & 17,748 & 79,048 & 0 & \\
\hline $1982 / 83$ & $1,189,581$ & $1,542,014$ & 0 & 82,298 & 65,410 & 19,951 & 85,361 & 0 & \\
\hline $1983 / 84$ & $1,206,471$ & $1,476,607$ & 0 & 72,378 & 70,965 & 20,588 & 91,553 & 0 & \\
\hline $1984 / 85$ & $1,207,889$ & $1,405,646$ & 0 & 64,603 & 87,580 & 20,637 & 108,217 & 0 & \\
\hline $1985 / 86$ & $1,184,914$ & $1,318,070$ & 0 & 53,566 & 93,787 & 19,938 & 113,725 & 0 & \\
\hline $1986 / 87$ & $1,144,699$ & $1,224,289$ & 0 & 28,793 & 104,700 & 20,242 & 124,942 & 0 & \\
\hline $1987 / 88$ & $1,068,793$ & $1,119,592$ & 0 & 22,222 & 100,995 & 18,202 & 119,197 & 0 & \\
\hline $1988 / 89$ & 990,023 & $1,018,599$ & 0 & 10,196 & 96,959 & 16,620 & 113,579 & 0 & as \\
\hline $1989 / 90$ & 903,262 & 921,643 & 0 & 10,196 & 93,611 & 14,821 & 108,432 & 0 & $\infty$ \\
\hline $1990 / 91$ & 819,849 & 828,034 & 0 & 8,185 & 79,187 & 13,055 & 92,242 & 0 & \\
\hline $1991 / 92$ & 748,853 & 748,853 & 0 & 0 & 75,606 & 11,577 & 87,183 & 0 & \\
\hline $1992 / 93$ & 673,251 & 673,251 & 0 & 0 & 72,922 & 10,085 & 83,007 & 0 & \\
\hline $1993 / 94$ & 600,332 & 600,332 & 0 & 0 & 57,264 & 8,565 & 65,829 & 0 & \\
\hline $1994 / 95$ & 543,075 & 543,075 & 0 & 0 & 55,327 & 7,553 & 62,880 & 0 & \\
\hline $1995 / 96$ & 487,752 & 487,752 & 0 & 0 & 52,007 & 6,588 & 58,595 & 0 & \\
\hline $1996 / 97$ & 435,747 & 435,747 & 0 & 0 & 37,225 & 4,426 & 41,651 & 0 & \\
\hline
\end{tabular}

[a] This column shows the amount of arithmetic imbalance in the amount outstanding including undisbursed from one year to the next

The most common causes of imbalances are changes in exchange rates and transfer of debts from one category to another in the table.

Source: "Afghanistan: The Journey to Economic Development", Vol.II, March 1978, World Bank 
Table A V-9. Service Payments, Commitments, Disbursements and Outstanding Amounts of External Public Debt

Projections Based on Debt Outstanding Including Undisbursed as of March 31, 1977

Debt Repayable in Foreign Currency and Goods

\section{SUPPLIERS CREDIT ONLY}

(thousands of U.S. dollars)

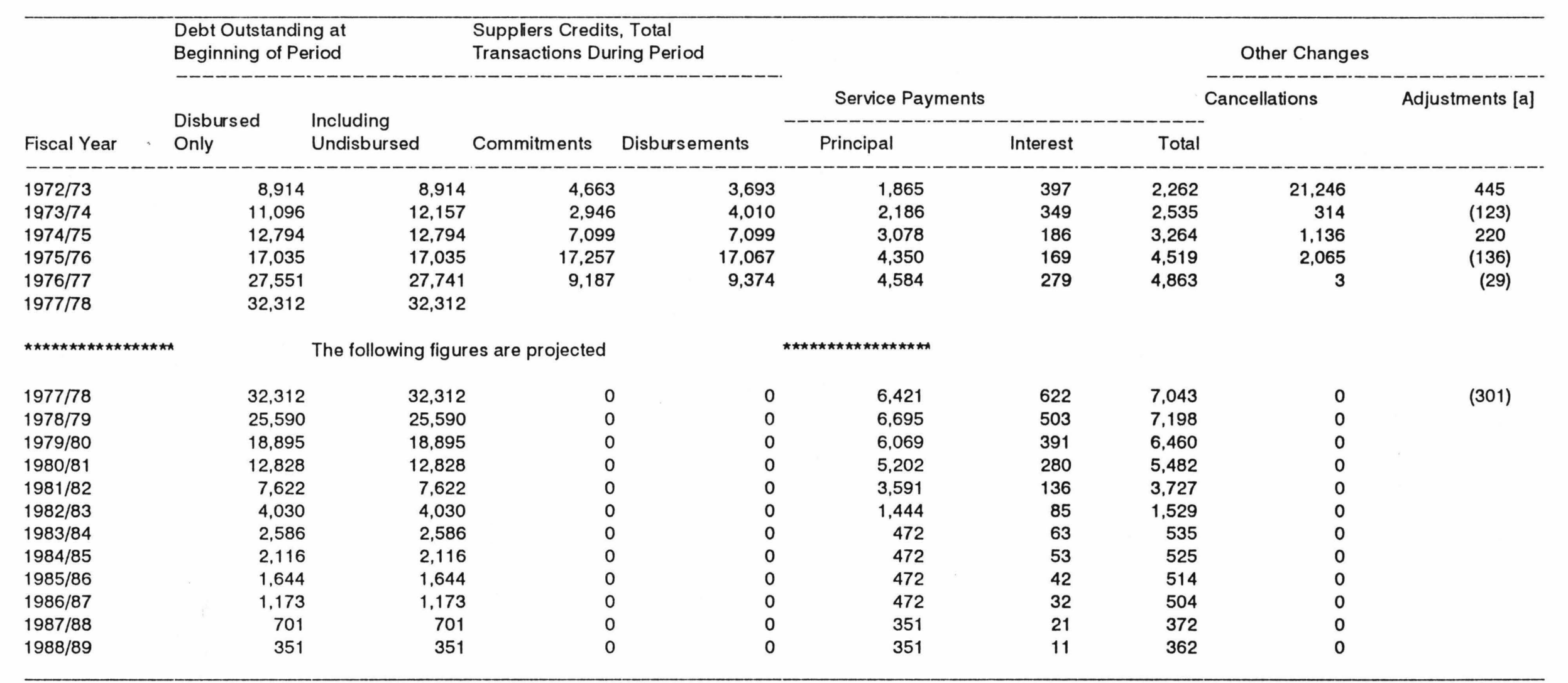

[a] This column shows the amount of arithmetic imbalance in the amount outstanding including undisbursed from one year to the next.

The most common causes of imbalances are changes in exchange rates and transfer of debts from one category to another in the table.

Source: "Afghanistan: The Journey to Economic Development", Vol. II, March 1978, World Bank. 
Table A V - 10. External Public Debt Outstanding Including Undisbursed as of Mar, 31, 1977, Debt Repayable in Foreign Currency and Goods

(thousands of U.S. dollars)

\begin{tabular}{|c|c|c|c|c|c|}
\hline & \multicolumn{2}{|c|}{ Debt Outstanding } & \multicolumn{3}{|c|}{ In Arrears } \\
\hline & Disbursed & Undisbursed & Total & Principal & Interest \\
\hline \multicolumn{6}{|l|}{ Suppliers Credits } \\
\hline France & 306 & 0 & 306 & 306 & 59 \\
\hline Rep. of Korea & 593 & 0 & 593 & 0 & 0 \\
\hline Kuwait & 180 & 0 & 180 & 0 & 0 \\
\hline United States & 120 & 0 & 120 & 0 & 0 \\
\hline USSR & 31,113 & 0 & 31,113 & 0 & 0 \\
\hline Total & 32,312 & 0 & 32,312 & 306 & 59 \\
\hline \multicolumn{6}{|l|}{ Multilateral Loans } \\
\hline $\mathrm{ADB}$ & 4,322 & 54,528 & 58,850 & 0 & 0 \\
\hline IDA & 33,461 & 70,373 & 103,834 & 0 & 0 \\
\hline OPEC Fund & 3,750 & 0 & 3,750 & 0 & 0 \\
\hline Total & 41,533 & 124,901 & 166,434 & 0 & 0 \\
\hline \multicolumn{6}{|l|}{ Bilateral Loans } \\
\hline Bulgaria & 0 & 10,000 & 10,000 & 0 & 0 \\
\hline People's Rep. of China & 14,211 & 57,990 & 72,201 & 0 & 0 \\
\hline Czechoslovakia & 6,827 & 43,173 & 50,000 & 0 & 0 \\
\hline Denmark & 3,406 & 12 & 3,418 & 0 & 0 \\
\hline Fed. Rep. of Germany & 69,052 & 20,648 & 89,700 & 0 & 0 \\
\hline Iran & 23,235 & 29,600 & 52,835 & 0 & 0 \\
\hline Iraq & 2,071 & 12,929 & 15,000 & 0 & 0 \\
\hline Japan & 1,748 & 497 & 2,245 & 0 & 0 \\
\hline Saudi Arabia & 3,039 & 51,961 & 55,000 & 0 & 0 \\
\hline United Kingdom & 839 & 1,034 & 1,873 & 0 & 0 \\
\hline United States & 105,207 & 3,678 & 108,885 & 0 & 0 \\
\hline USSR & 605,328 & 480,919 & $1,086,247$ & 0 & 0 \\
\hline Yugoslavia & 2,380 & 0 & 2,380 & 0 & 0 \\
\hline Total & 837,343 & 712,441 & $1,549,784$ & 0 & 0 \\
\hline Total External Public Debt & 911,188 & 837,342 & $1,748,530$ & 306 & 59 \\
\hline
\end{tabular}

Notes: Only debts with an original or extended maturity of over one year are

included in this table. Debt outstanding includes principal in arrears but excludes

interest in arrears.

Source: "Afghanistan: The Journey to Economic Development", Vol. II, March 1978, World Bank. 
Table A V - 11. International Reserves and Related Items

(millions of U.S. dollars)

\begin{tabular}{|c|c|c|c|c|c|c|c|c|c|}
\hline & $1971 / 72$ & $1972 / 73$ & $1973 / 74$ & $1974 / 75$ & $1975 / 76$ & June & $\begin{array}{l}1976 \\
\text { September }\end{array}$ & December & $\begin{array}{l}1977 \text { [a] } \\
\quad \text { March } 20\end{array}$ \\
\hline Da Afghanistan Bank & 49.39 & 49.25 & 59.92 & 82.38 & 140.10 & 127.98 & 156.86 & 196.66 & 205.36 \\
\hline International reserves & 61.90 & 58.80 & 59.41 & 75.84 & 125.73 & 116.65 & 127.56 & 168.66 & 172.60 \\
\hline Gold & 35.42 & 39.35 & 39.35 & 40.69 & 37.66 & 37.20 & 37.61 & 37.75 & 38.43 \\
\hline SDRs & 4.96 & 1.36 & 5.09 & 6.33 & 6.21 & 6.00 & 5.96 & 5.94 & 5.70 \\
\hline Reserve position in IMF & 0.00 & 0.00 & 0.00 & 0.00 & 0.00 & 0.00 & 0.00 & 0.00 & 0.00 \\
\hline Convertible For. exchange & 21.52 & 18.09 & 14.97 & 28.82 & 81.86 & 73.45 & 83.99 & 124.97 & 128.47 \\
\hline Inconvertible exchange & 0.99 & 0.88 & 1.93 & 2.37 & 3.32 & 5.00 & 6.84 & 8.31 & 8.04 \\
\hline Net balance under bilateral & & & & & & & & & \\
\hline payments agreements & -5.11 & -3.52 & 6.10 & 7.59 & 21.72 & 16.93 & 24.46 & 21.69 & 17.22 \\
\hline Use of fund credit & -8.39 & -6.91 & -7.52 & -3.42 & -10.67 & -10.60 & -2.00 & -2.00 & 0.00 \\
\hline IBRD bonds & 0.00 & 0.00 & 0.00 & 0.00 & 0.00 & 0.00 & 0.00 & 0.00 & 7.50 \\
\hline Foreign exchange of other banks & 4.14 & 6.50 & 9.64 & 12.44 & 7.52 & 9.42 & 10.04 & 10.36 & 10.00 \\
\hline Convertible exchange & 3.05 & 5.91 & 9.31 & 11.59 & 6.41 & 7.61 & 7.75 & 7.66 & -- \\
\hline Inconvertible exchange & 1.09 & 0.59 & 0.33 & 0.85 & 1.11 & 1.81 & 2.29 & 2.70 & -- \\
\hline Total & 53.53 & 55.75 & 69.56 & 94.82 & 147.62 & 137.40 & 166.90 & 207.02 & 215.36 \\
\hline
\end{tabular}

[a] Preliminary

[b] Estimate

Source: GOA Da Afghanistan Bank; cited from "Afghanistan: The Journey to Economic Development", Vol. II, March 1978, World Bank. 
Table A V-12. Principal Exports by Commodity (IMF Series)

(millions of U.S. dollars)

\begin{tabular}{|c|c|c|c|c|c|c|c|c|c|}
\hline & $1977 / 78$ & $1978 / 79$ & $1979 / 80$ & $1980 / 81$ & $1981 / 82$ & $1982 / 83$ & $1983 / 84$ & $1984 / 85$ & $1985 / 86$ \\
\hline Natural gas & 39.3 & 53.0 & 102.9 & 233.1 & 272.6 & 283.6 & 305.3 & 314.3 & 309.4 \\
\hline Dried fruits & 84.2 & 100.3 & 175.4 & 169.5 & 171.4 & 154.0 & 192.0 & 99.3 & 94.5 \\
\hline Fresh fruits & 21.4 & 26.2 & 31.2 & 39.8 & 50.9 & 55.2 & 66.4 & 46.8 & 26.2 \\
\hline Carpets and rugs & 37.8 & 36.6 & 62.9 & 103.6 & 69.3 & 80.8 & 50.4 & 42.2 & 26.8 \\
\hline Cotton & 53.2 & 35.8 & 36.8 & 39.7 & 22.6 & 9.0 & 10.2 & 22.4 & 19.5 \\
\hline Wool & 5.2 & 6.1 & 11.8 & 12.3 & 25.6 & 20.5 & 25.4 & 26.1 & 22.5 \\
\hline Karakul skins & 18.7 & 18.6 & 24.9 & 33.3 & 19.8 & 21.5 & 9.6 & 9.2 & 5.7 \\
\hline Other exports & 54.6 & 45.2 & 47.8 & 73.9 & 58.6 & 83.1 & 69.3 & 72.6 & 62.2 \\
\hline Total & 314.4 & 321.8 & 493.7 & 705.2 & 690.8 & 707.7 & 728.6 & 632.9 & 566.8 \\
\hline
\end{tabular}

Source: GOA Da Afghanistan Bank, Central Statistical Office; cited from Afghanistan: Recent

Economic Developments, IMF, 1983-86.

Table A V -12. Principal Exports by Commodity (IMF Series)

(millions of U.S. dollars)

\begin{tabular}{|c|c|c|c|c|c|c|c|c|c|}
\hline & Actual & $\begin{array}{l}1977 / 78 \\
\text { cor. fac. }\end{array}$ & adj. fig. & Actual & $\begin{array}{l}1978 / 79 \\
\text { cor. fac. }\end{array}$ & adj. fig. & Actual & $\begin{array}{l}1979 / 80 \\
\text { cor. fac. }\end{array}$ & adj. fig. \\
\hline Natural gas & 39.3 & 1.00 & 39.3 & 53.0 & 1.00 & 53.0 & 102.9 & 1.00 & 102.9 \\
\hline Dried fruits & 84.2 & 1.00 & 84.2 & 100.3 & 1.00 & 100.3 & 175.4 & 1.00 & 175.4 \\
\hline Fresh fruits & 21.4 & 1.00 & 21.4 & 26.2 & 1.00 & 26.2 & 31.2 & 1.00 & 31.2 \\
\hline Carpets and rugs & 37.8 & 1.00 & 37.8 & 36.6 & 1.00 & 36.6 & 62.9 & 1.00 & 62.9 \\
\hline Cotton & 53.2 & 1.00 & 53.2 & 35.8 & 1.00 & 35.8 & 36.8 & 1.00 & 36.8 \\
\hline Wool & 5.2 & 1.00 & 5.2 & 6.1 & 1.00 & 6.1 & 11.8 & 1.00 & 11.8 \\
\hline Karakul skins & 18.7 & 1.00 & 18.7 & 18.6 & 1.00 & 18.6 & 24.9 & 1.00 & 24.9 \\
\hline Other exports & 54.6 & 1.00 & 54.6 & 45.2 & 1.00 & 45.2 & 47.8 & 1.00 & 47.8 \\
\hline Total & 314.4 & & 314.4 & 321.8 & & 321.8 & 493.7 & & 493.7 \\
\hline
\end{tabular}

Source: GOA Da Afghanistan Bank, Central Statistical Office; cited from Afghanistan: Recent

Economic Developments, IMF, 1983-86. 
Table AV-13. Merchandise Exports by Commodity

\begin{tabular}{|c|c|c|c|c|c|}
\hline Commodity & & 1978/9 & 1984/85 & $1985 / 86$ & 1986,87 \\
\hline 1. Natural Gas & $\begin{array}{l}\text { Millon Cubic Mot. } \\
\$ 5000\end{array}$ & $\begin{array}{r}2,200 \\
53,018\end{array}$ & $\begin{array}{r}2,440 \\
314,326\end{array}$ & $\begin{array}{r}2,300 \\
309,388\end{array}$ & $\begin{array}{r}2,100 \\
259579\end{array}$ \\
\hline 2. Chomical Forrlizor & $\begin{array}{l}\text { Thousand Tons } \\
\text { so00 }\end{array}$ & $=-$ & $\begin{array}{r}188 \\
2941\end{array}$ & $\begin{array}{r}30.0 \\
4,820\end{array}$ & $\begin{array}{r}30.0 \\
5,400\end{array}$ \\
\hline 3. Fresh Fruits & $\begin{array}{l}\text { Thousand Tons } \\
\$ 000\end{array}$ & $\begin{array}{r}88.9 \\
26,209\end{array}$ & $\begin{array}{r}98.8 \\
46,844\end{array}$ & $\begin{array}{r}69.2 \\
26,150\end{array}$ & $\begin{array}{r}61.2 \\
24,092\end{array}$ \\
\hline 4. Dry Fruits & $\begin{array}{l}\text { Thousand Tons } \\
\text { soov }\end{array}$ & $\begin{array}{r}85.2 \\
100317\end{array}$ & $\begin{array}{r}80.5 \\
99,335\end{array}$ & $\begin{array}{r}63.4 \\
94,501\end{array}$ & $\begin{array}{r}86.5 \\
134878\end{array}$ \\
\hline 5. Oil Seods & $\begin{array}{l}\text { Thousand Tons } \\
\text { sooo }\end{array}$ & $\begin{array}{r}7.5 \\
3,997\end{array}$ & $\begin{array}{r}2.1 \\
2.652\end{array}$ & $\begin{array}{r}1.0 \\
959\end{array}$ & $\begin{array}{l}0.8 \\
719\end{array}$ \\
\hline 8. Leather (skins) & $\begin{array}{l}\text { Thousand Skins } \\
\text { sooo }\end{array}$ & $\begin{array}{r}3,782 \\
14,148\end{array}$ & $\begin{array}{r}3,482 \\
25,798\end{array}$ & 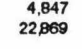 & $\begin{array}{r}4,374 \\
31,109\end{array}$ \\
\hline 7. Karakul & $\begin{array}{l}\text { Thousand Polts } \\
\text { so00 }\end{array}$ & $\begin{array}{r}999 \\
18561\end{array}$ & $\begin{array}{l}4,76 \\
9,197\end{array}$ & $\begin{array}{l}762 \\
\mathbf{5 , 7 0 5}\end{array}$ & $\begin{array}{r}809 \\
10,488\end{array}$ \\
\hline 8. Wool & $\begin{array}{l}\text { Thousand Tons } \\
\text { so00 }\end{array}$ & $\begin{array}{r}3.9 \\
6,110\end{array}$ & $\begin{array}{r}8.3 \\
26,067\end{array}$ & $\begin{array}{r}10.5 \\
22,450\end{array}$ & $\begin{array}{r}5.7 \\
14.582\end{array}$ \\
\hline 9. Ginned Cotton & $\begin{array}{l}\text { Thousand Tons } \\
\text { so00 }\end{array}$ & $\begin{array}{r}27,3 \\
35,752\end{array}$ & $\begin{array}{r}13,4 \\
22,430\end{array}$ & $\begin{array}{r}11.7 \\
19530\end{array}$ & $\begin{array}{r}7.6 \\
9,799\end{array}$ \\
\hline 10. Medical Herbs & $\begin{array}{l}\text { Thousand Tons } \\
\text { soo0 }\end{array}$ & $\begin{array}{r}13.7 \\
8,679\end{array}$ & $\begin{array}{r}109 \\
18383\end{array}$ & $\begin{array}{r}17.1 \\
21,537\end{array}$ & $\begin{array}{r}8.6 \\
9,513\end{array}$ \\
\hline 11. Carpots \& Rugs & $\begin{array}{l}\text { Thousand Sq.M. } \\
\$ 000\end{array}$ & $\begin{array}{r}559 \\
36,575\end{array}$ & $\begin{array}{r}62,23 \\
42,228\end{array}$ & $\begin{array}{r}603 \\
28,759\end{array}$ & $\begin{array}{r}769 \\
39,524\end{array}$ \\
\hline 12. Fur coats & $\begin{array}{l}\text { Thousand Pioces } \\
\text { s000 }\end{array}$ & $\begin{array}{l}290 \\
473\end{array}$ & $\begin{array}{l}7.0 \\
80\end{array}$ & $\begin{array}{l}20 \\
25\end{array}$ & $\begin{array}{l}20 \\
118\end{array}$ \\
\hline 13. Casings & $\begin{array}{l}\text { Thousand Pings } \\
\text { soo0 }\end{array}$ & $\begin{array}{l}2,283 \\
4,733\end{array}$ & $\begin{array}{l}1,667 \\
\mathbf{3 , 3 4 4}\end{array}$ & $\begin{array}{l}2008 \\
2,344\end{array}$ & $\begin{array}{l}2,002 \\
2,484\end{array}$ \\
\hline Other Commodifes & 5000 & 14,203 & 21,243 & 9,796 & 9,633 \\
\hline Total Exports & $\$ 000$ & 322,773 & 632,878 & 566,831 & 551,918 \\
\hline
\end{tabular}

of April 1988 .
Table A V - 13. Merchandise Exports by Commodity

\begin{tabular}{|c|c|c|c|c|c|c|c|c|c|c|c|c|c|}
\hline Commodity & & Actual & 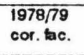 & adj. fig. & Actual & 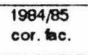 & adj. fg. & Actual & 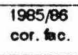 & adj. fig. & Actual & $\begin{array}{l}\begin{array}{l}1986,87 \\
\text { cor.tro. }\end{array}\end{array}$ & adj. ig \\
\hline 1. Natural Gas & $\begin{array}{l}\text { Millon Cubic Met. } \\
\text { sooo }\end{array}$ & $\begin{array}{r}2,200 \\
53,018\end{array}$ & $\begin{array}{l}1.00 \\
1.00\end{array}$ & $\begin{array}{r}2,200 \\
53,018\end{array}$ & $\begin{array}{r}2,440 \\
314,326\end{array}$ & $\begin{array}{l}100 \\
100\end{array}$ & $\begin{array}{r}2,440 \\
314,326\end{array}$ & $\begin{array}{r}2380 \\
309,386\end{array}$ & $\begin{array}{l}100 \\
100\end{array}$ & $\begin{array}{r}2380 \\
309,386\end{array}$ & $\begin{array}{r}2,100 \\
259579\end{array}$ & $\begin{array}{l}100 \\
100\end{array}$ & $\begin{array}{r}2.100 \\
259.579\end{array}$ \\
\hline 2. Chemical Forflizor & $\begin{array}{l}\text { Thousand Tons } \\
\text { soov }\end{array}$ & $=$ & $\begin{array}{l}1.00 \\
100\end{array}$ & $\stackrel{0}{0}$ & $\begin{array}{r}188 \\
2941\end{array}$ & $\begin{array}{l}100 \\
100\end{array}$ & $\begin{array}{r}19 \\
2941\end{array}$ & $\begin{array}{r}30.0 \\
4,820\end{array}$ & $\begin{array}{l}1.00 \\
100\end{array}$ & $\begin{array}{r}30 \\
4,820\end{array}$ & $\begin{array}{r}30.0 \\
5,400\end{array}$ & $\begin{array}{l}1.00 \\
100\end{array}$ & $\begin{array}{r}30 \\
5,400\end{array}$ \\
\hline 3. Frosh Fruits & $\begin{array}{l}\text { Thousand Tons } \\
\text { sooo }\end{array}$ & $\begin{array}{r}88.9 \\
26,209\end{array}$ & $\begin{array}{l}100 \\
1.00\end{array}$ & $\begin{array}{r}88.9 \\
26,209\end{array}$ & $\begin{array}{r}98.8 \\
46,844\end{array}$ & $\begin{array}{l}1.00 \\
100\end{array}$ & $\begin{array}{r}98.8 \\
46,844\end{array}$ & $\begin{array}{r}69.2 \\
26.150\end{array}$ & $\begin{array}{l}1.00 \\
100\end{array}$ & $\begin{array}{r}69.2 \\
26,150\end{array}$ & $\begin{array}{r}61.2 \\
24,092\end{array}$ & $\begin{array}{l}1.00 \\
1.00\end{array}$ & $\begin{array}{r}81.2 \\
24,092\end{array}$ \\
\hline 4. Dry Fruits & $\begin{array}{l}\text { Thousand Tons } \\
\text { sooo }\end{array}$ & $\begin{array}{r}85.2 \\
100317\end{array}$ & $\begin{array}{l}100 \\
1.00\end{array}$ & $\begin{array}{r}85.2 \\
100317\end{array}$ & $\begin{array}{r}80.5 \\
99,335\end{array}$ & $\begin{array}{l}100 \\
100\end{array}$ & $\begin{array}{r}80.5 \\
99,335\end{array}$ & $\begin{array}{r}63.4 \\
94,501\end{array}$ & $\begin{array}{l}100 \\
100\end{array}$ & $\begin{array}{r}63.4 \\
94,501\end{array}$ & $\begin{array}{r}86.5 \\
134878\end{array}$ & $\begin{array}{l}1.00 \\
1.00\end{array}$ & $\begin{array}{r}86.5 \\
134878\end{array}$ \\
\hline 5. Oil Soods & $\begin{array}{l}\text { Thousand Tons } \\
\text { sooo }\end{array}$ & $\begin{array}{r}7.5 \\
3,997\end{array}$ & $\begin{array}{l}1.00 \\
100\end{array}$ & $\begin{array}{r}7.5 \\
3,997\end{array}$ & $\begin{array}{r}2.1 \\
2852\end{array}$ & $\begin{array}{l}100 \\
100\end{array}$ & $\begin{array}{r}2.1 \\
2652\end{array}$ & $\begin{array}{l}10 \\
859\end{array}$ & $\begin{array}{l}100 \\
100\end{array}$ & $\begin{array}{l}10 \\
959\end{array}$ & $\begin{array}{l}0.8 \\
719\end{array}$ & $\begin{array}{l}100 \\
1.00\end{array}$ & $\begin{array}{l}0.8 \\
719\end{array}$ \\
\hline 6. Leathor (skins) & $\begin{array}{l}\text { Thousand Skins } \\
\$ 000\end{array}$ & $\begin{array}{r}3,782 \\
14,146\end{array}$ & $\begin{array}{l}1.00 \\
1.00\end{array}$ & $\begin{array}{r}3.782 \\
14.146\end{array}$ & $\begin{array}{r}3,482 \\
25,798\end{array}$ & $\begin{array}{l}100 \\
100\end{array}$ & $\begin{array}{r}3,482 \\
25,798\end{array}$ & $\begin{array}{r}4.847 \\
22.869\end{array}$ & $\begin{array}{l}100 \\
1.00\end{array}$ & $\begin{array}{r}4.847 \\
22.869\end{array}$ & $\begin{array}{r}4,374 \\
31,109\end{array}$ & $\begin{array}{l}1.00 \\
1.00\end{array}$ & $\begin{array}{r}4,374 \\
31,109\end{array}$ \\
\hline 7. Karakul & $\begin{array}{l}\text { Thousand Polts } \\
\$ 000\end{array}$ & $\begin{array}{r}999 \\
18561\end{array}$ & $\begin{array}{l}1.00 \\
1.00\end{array}$ & $\begin{array}{r}999 \\
18561\end{array}$ & $\begin{array}{r}476 \\
9,197\end{array}$ & $\begin{array}{l}100 \\
1.00\end{array}$ & $\begin{array}{r}476 \\
9,197\end{array}$ & $\begin{array}{r}762 \\
5,705\end{array}$ & $\begin{array}{l}1.00 \\
100\end{array}$ & $\begin{array}{r}762 \\
5,705\end{array}$ & $\begin{array}{r}809 \\
10,488\end{array}$ & $\begin{array}{l}1.00 \\
1.00\end{array}$ & $\begin{array}{r}809 \\
10,488\end{array}$ \\
\hline 8. Wool & $\begin{array}{l}\text { Thousand Tons } \\
\text { sooo }\end{array}$ & $\begin{array}{r}3.9 \\
6,110\end{array}$ & $\begin{array}{l}1.00 \\
1.00\end{array}$ & $\begin{array}{r}3.9 \\
6,110\end{array}$ & $\begin{array}{r}8.3 \\
28,067\end{array}$ & $\begin{array}{l}100 \\
100\end{array}$ & $\begin{array}{r}8.3 \\
26.067\end{array}$ & $\begin{array}{r}105 \\
22,450\end{array}$ & $\begin{array}{l}1.00 \\
1.00\end{array}$ & $\begin{array}{r}105 \\
22,450\end{array}$ & $\begin{array}{r}5.7 \\
14582\end{array}$ & $\begin{array}{l}1.00 \\
1.00\end{array}$ & $\begin{array}{r}5.7 \\
14582\end{array}$ \\
\hline 9. Ginned Cotton & $\begin{array}{l}\text { Thousand Tons } \\
\text { sooo }\end{array}$ & $\begin{array}{r}273 \\
35,752\end{array}$ & $\begin{array}{l}100 \\
1.00\end{array}$ & $\begin{array}{r}27,3 \\
35,752\end{array}$ & $\begin{array}{r}13,4 \\
22,430\end{array}$ & $\begin{array}{l}100 \\
100\end{array}$ & $\begin{array}{r}13,4 \\
22,430\end{array}$ & $\begin{array}{r}11.7 \\
19530\end{array}$ & $\begin{array}{l}100 \\
100\end{array}$ & $\begin{array}{l}111.7 \\
19530\end{array}$ & $\begin{array}{r}7.8 \\
9,799\end{array}$ & $\begin{array}{l}1.00 \\
1.00\end{array}$ & $\begin{array}{l}7,6 \\
9,799\end{array}$ \\
\hline 10. Medical Herbs & $\begin{array}{l}\text { Thousand Tons } \\
\text { sooo }\end{array}$ & $\begin{array}{l}\mathbf{1 3 . 7} \\
8,679\end{array}$ & $\begin{array}{l}1.00 \\
1.00\end{array}$ & $\begin{array}{r}13.7 \\
8,679\end{array}$ & $\begin{array}{r}109 \\
18,383\end{array}$ & $\begin{array}{l}100 \\
100\end{array}$ & $\begin{array}{r}109 \\
16,383\end{array}$ & $\begin{array}{r}17.1 \\
21537\end{array}$ & $\begin{array}{l}100 \\
1.00\end{array}$ & $\begin{array}{r}17.1 \\
21537\end{array}$ & $\begin{array}{r}8.6 \\
9,513\end{array}$ & $\begin{array}{l}100 \\
1.00\end{array}$ & $\begin{array}{r}8.6 \\
9,513\end{array}$ \\
\hline 11. Carpots \& Augs & $\begin{array}{l}\text { Thousand Sq.M. } \\
\text { sooo }\end{array}$ & $\begin{array}{l}529 \\
36,575\end{array}$ & $\begin{array}{l}100 \\
100\end{array}$ & $\begin{array}{r}529 \\
36,575\end{array}$ & $\begin{array}{r}663 \\
42,228\end{array}$ & $\begin{array}{l}100 \\
100\end{array}$ & $\begin{array}{r}663 \\
42,228\end{array}$ & $\begin{array}{r}603 \\
26,759\end{array}$ & $\begin{array}{l}100 \\
100\end{array}$ & $\begin{array}{r}603 \\
26,759\end{array}$ & $\begin{array}{r}769 \\
39,524\end{array}$ & $\begin{array}{l}100 \\
1.00\end{array}$ & $\begin{array}{r}769 \\
39,524\end{array}$ \\
\hline 12. Fur coats & $\begin{array}{l}\text { Thousand Pieces } \\
\$ 000\end{array}$ & $\begin{array}{l}290 \\
473\end{array}$ & $\begin{array}{l}100 \\
100\end{array}$ & $\begin{array}{l}290 \\
473\end{array}$ & $\begin{array}{l}7.0 \\
90\end{array}$ & $\begin{array}{l}100 \\
100\end{array}$ & $\begin{array}{r}7.0 \\
90\end{array}$ & $\begin{array}{l}20 \\
25\end{array}$ & $\begin{array}{l}100 \\
100\end{array}$ & $\begin{array}{l}20 \\
25\end{array}$ & $\begin{array}{l}20 \\
118\end{array}$ & $\begin{array}{l}100 \\
1.00\end{array}$ & $\begin{array}{l}20 \\
118\end{array}$ \\
\hline 13. Casings & $\begin{array}{l}\text { Thousand Rings } \\
\text { soos }\end{array}$ & $\begin{array}{l}2,228 \\
4,733\end{array}$ & $\begin{array}{l}100 \\
100\end{array}$ & $\begin{array}{l}2,228 \\
4,733\end{array}$ & $\begin{array}{l}1,687 \\
3,344\end{array}$ & $\begin{array}{l}1.00 \\
100\end{array}$ & $\begin{array}{l}1,667 \\
{ }_{3,344}\end{array}$ & $\begin{array}{l}2,008 \\
2,344\end{array}$ & $\begin{array}{l}100 \\
100\end{array}$ & $\begin{array}{l}20089 \\
2344\end{array}$ & $\begin{array}{l}2,002 \\
2,484\end{array}$ & $\begin{array}{l}100 \\
100\end{array}$ & $\begin{array}{l}2,002 \\
2,484\end{array}$ \\
\hline Other Commoditos & $\$ 000$ & 14,203 & 100 & 14,203 & 21,243 & 1.00 & 21,243 & 9,796 & 100 & 9,796 & 9,633 & 100 & 9.633 \\
\hline Totan Exports & 5000 & 322,773 & & 322,773 & 632,878 & & 622.878 & 566,831 & & 566,831 & 551,918 & & 551,918 \\
\hline
\end{tabular}

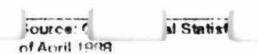


Table A V-14. Principal Exports by Commodity (World Bank Series)

(millions of U.S. dollars)

\begin{tabular}{|c|c|c|c|c|c|c|c|c|c|c|}
\hline & $1966 / 67$ & $1967 / 68$ & $1968 / 69$ & $1969 / 70$ & $1970 / 71$ & $1971 / 72$ & $1972 / 73$ & $1973 / 74$ & $1974 / 75$ & $1975 / 76$ \\
\hline Food \& live animals & 23.5 & 27.5 & 28.2 & 29.5 & 30.4 & 29.1 & 45.7 & 73.6 & 96.0 & 76.7 \\
\hline Fresh fruits & 6.6 & 8.1 & 8.0 & 8.9 & 8.3 & 8.2 & 10.7 & 21.6 & 31.5 & 20.1 \\
\hline Dry fruits & 14.9 & 17.1 & 17.5 & 17.6 & 20.8 & 17.9 & 30.5 & 44.6 & 58.2 & 54.7 \\
\hline Other & 2.0 & 2.3 & 2.7 & 3.0 & 1.3 & 3.0 & 4.5 & 7.4 & 6.3 & 1.9 \\
\hline Crude materials inedible except fuel & 33.1 & 30.5 & 29.2 & 32.8 & 31.2 & 43.3 & 42.9 & 47.4 & 78.3 & 86.9 \\
\hline Karakul & 11.9 & 12.4 & 7.2 & 13.1 & 8.5 & 16.4 & 16.1 & 17.0 & 12.7 & 10.4 \\
\hline Other skins \& furs & 2.2 & 4.0 & 3.4 & 2.8 & 3.9 & 4.6 & 3.3 & 6.9 & 6.7 & 11.7 \\
\hline Ọil seeds & 0.3 & 0.5 & 1.8 & 2.6 & 0.7 & & 0.6 & 2.5 & 5.5 & 8.3 \\
\hline W̉ool \& hair & 5.0 & 4.9 & 7.0 & 7.0 & 7.5 & 7.7 & 5.9 & 5.8 & 6.5 & 10.8 \\
\hline Cotton & 11.9 & 7.9 & 5.9 & 5.7 & 8.5 & 12.4 & 11.1 & 7.2 & 34.7 & 35.3 \\
\hline Licorice root & & 0.1 & 0.5 & & 0.3 & 0.9 & 0.6 & 1.8 & 7.1 & 5.3 \\
\hline Other & 1.8 & 0.7 & 3.4 & 1.6 & 1.8 & 1.3 & 5.3 & 6.2 & 5.1 & 5.1 \\
\hline Natural gas & & 2.9 & 9.0 & 12.1 & 14.0 & 14.5 & 17.1 & 18.0 & 32.1 & 46.3 \\
\hline Chemicals & & & & & & & 0.5 & & 0.1 & 0.1 \\
\hline \multicolumn{11}{|l|}{ Manufactured goods classified } \\
\hline chiefly by material & 8.0 & 5.3 & 5.2 & 7.1 & 9.3 & 12.7 & 18.0 & 19.2 & 24.1 & 24.3 \\
\hline Carpets \& rugs & 8.0 & 5.1 & 4.5 & 6.2 & 6.6 & 8.0 & 10.8 & 14.5 & 19.9 & 16.1 \\
\hline Other & & 0.2 & 0.7 & 0.9 & 2.7 & 4.7 & 7.2 & 4.7 & 4.2 & 8.2 \\
\hline $\begin{array}{l}\text { Commodities \& transactions not } \\
\text { classified according to kind }\end{array}$ & 0.1 & 0.2 & 0.2 & 0.2 & 0.2 & 0.1 & 0.3 & 0.9 & & 1.2 \\
\hline Total Exports & 64.7 & 66.4 & 71.8 & 81.7 & 85.1 & 99.7 & 124.5 & 159.1 & 230.6 & 235.5 \\
\hline
\end{tabular}

Sources: GOA Da Afghanistan Bank and Central Statistical Office; cited from "Afghanistan: The Journey to Economic Development", Vol. II, March 1978, World Bank. 
Table A V-15. Exports by Region

(millions of U.S. dollars)

\begin{tabular}{|c|c|c|c|c|c|c|c|c|c|}
\hline & $1966 / 67$ & $1967 / 68$ & $1968 / 69$ & $1969 / 70$ & $1970 / 71$ & $1971 / 72$ & $1972 / 73$ & $1973 / 74$ & $1974 / 75$ \\
\hline Multilateral Countries & & & & & & & $\checkmark$ & & \\
\hline \multicolumn{10}{|l|}{ OECD Countries } \\
\hline UK & 9.4 & 10.6 & 7.4 & 12.8 & 13.8 & 19.8 & 19.9 & 23.3 & 23.0 \\
\hline Germany & 3.7 & 0.6 & 1.3 & 1.6 & 4.4 & 8.6 & 7.7 & 6.9 & 5.7 \\
\hline Japan & 0.1 & 0.2 & 0.1 & 0.1 & 0.2 & 0.4 & 0.1 & 0.6 & 1.0 \\
\hline Other & 5.3 & 5.5 & 5.3 & 7.5 & 7.4 & 11.4 & 13.2 & 15.2 & 18.4 \\
\hline Total OECD & 24.0 & 22.5 & 18.7 & 24.5 & 28.3 & 42.4 & 43.4 & 48.1 & 53.3 \\
\hline \multicolumn{10}{|l|}{ Other multilateral } \\
\hline India & 8.5 & 10.8 & 15.8 & 15.9 & 13.3 & 4.8 & 30.4 & 27.2 & 37.7 \\
\hline Pakistan & 5.4 & 5.5 & 5.7 & 5.3 & 6.0 & 5.2 & 5.3 & 23.0 & 26.2 \\
\hline Iran & 0.8 & 0.7 & 0.8 & 1.0 & 0.6 & 0.3 & 0.8 & 2.0 & 5.2 \\
\hline Other & 1.2 & 2.1 & 2.6 & 2.0 & 1.8 & 6.1 & 4.2 & 5.3 & 10.3 \\
\hline Total other multilateral & 15.9 & 19.1 & 24.9 & 24.2 & 21.7 & 16.4 & 40.7 & 57.5 & 79.4 \\
\hline \multicolumn{10}{|l|}{ Bilateral countries } \\
\hline USSR & 21.5 & 22.0 & 26.6 & 30.8 & 32.9 & 38.5 & 36.1 & 48.6 & 91.9 \\
\hline China & 1.0 & 0.4 & & 0.5 & 1.2 & 1.1 & 1.1 & 3.4 & 2.9 \\
\hline Other & 2.3 & 2.4 & 1.6 & 1.7 & 1.0 & 1.3 & 3.2 & 1.5 & 3.1 \\
\hline Total bilateral & 24.8 & 24.8 & 28.2 & 33.0 & 35.1 & 40.9 & 40.4 & 53.5 & 97.9 \\
\hline Total recorded exports & 64.7 & 66.4 & 71.8 & 81.7 & 85.1 & 99.7 & 124.5 & 159.1 & 230.6 \\
\hline
\end{tabular}

Sources: GOA Da Afghanistan Bank and Central Statistical Office; cited from "Afghanistan: The Journey to Economic Development",

Vol. II, March 1978, World Bank. 
Table A V-16. Merchandise Exports, 1976/77 \& 82/83, Seven-Year Plan

\begin{tabular}{|c|c|c|c|c|c|c|}
\hline Commodity & $\begin{array}{l}\text { Unit of } \\
\text { Measurement }\end{array}$ & $\begin{array}{r}\text { Total exports } \\
\text { in years } \\
1969 / 70- \\
1975 / 76\end{array}$ & $\begin{array}{l}\text { Total Seven } \\
\text { Year Plan } \\
\text { (Proj.) }\end{array}$ & $\begin{array}{c}\text { Of which years } \\
1976 / 77\end{array}$ & $1982 / 83$ & $\begin{array}{c}\text { Seven-yr. } \\
\text { Plan as \% of } \\
\text {-yrs. } 1969 / 70 \\
-1975 / 76\end{array}$ \\
\hline \multirow[t]{2}{*}{ Natural gas } & Bill. cubic meters & 18.30 & 18.30 & 2.50 & 3.20 & $100.0 \%$ \\
\hline & $(\$ 000,000)$ & 153.40 & 294.63 & 40.25 & 51.52 & $192.1 \%$ \\
\hline \multirow[t]{2}{*}{ Citrus } & Thousand tons & 13.70 & 49.05 & 5.17 & 9.16 & $358.0 \%$ \\
\hline & $(\$ 000,000)$ & 38.22 & 10.80 & 1.14 & 2.02 & $28.3 \%$ \\
\hline \multirow[t]{2}{*}{ Lapis lazuli } & Tons & 9.00 & 14.00 & 2.00 & 2.00 & $155.6 \%$ \\
\hline & $(\$ 000,000)$ & 0.81 & 5.60 & 0.80 & 0.80 & $694.8 \%$ \\
\hline \multirow[t]{2}{*}{ Barite } & Thousand tons & 5.00 & 155.00 & 10.00 & 30.00 & $3100.0 \%$ \\
\hline & $(\$ 000,000)$ & 0.12 & 3.88 & 0.25 & 0.75 & $3125.0 \%$ \\
\hline \multirow{2}{*}{ Chemical fertilizer } & Thousand tons & 6.00 & 50.00 & 30.00 & 0.00 & $833.3 \%$ \\
\hline & $(\$ 000,000)$ & 0.70 & 5.85 & 3.51 & 0.00 & $833.3 \%$ \\
\hline \multirow[t]{2}{*}{ Talc } & Thousand tons & 0.00 & 92.00 & 6.00 & 18.00 & \\
\hline & $(\$ 000,000)$ & 0.00 & 3.68 & 0.24 & 0.72 & \\
\hline \multirow[t]{2}{*}{ Casings } & Thousand rings & 11539.30 & 16480.00 & 2200.00 & 2500.00 & $142.8 \%$ \\
\hline & $(\$ 000,000)$ & 11.58 & 14.81 & 1.98 & 2.25 & $127.8 \%$ \\
\hline \multirow[t]{2}{*}{ Fresh fruit } & Thousand tons & 652.20 & 1295.00 & 136.00 & 242.00 & $198.6 \%$ \\
\hline & $(\$ 000,000)$ & 112.98 & 259.00 & 27.20 & 48.40 & $229.2 \%$ \\
\hline \multirow[t]{2}{*}{ Dry fruit } & Thousand tons & 342.40 & 513.00 & 62.00 & 87.00 & $149.8 \%$ \\
\hline & $(\$ 000,000)$ & 245.30 & 359.10 & 43.40 & 60.90 & $146.4 \%$ \\
\hline \multirow[t]{2}{*}{ Oil seeds } & Thousand tons & 65.90 & 84.00 & 12.00 & 12.00 & $127.5 \%$ \\
\hline & $(\$ 000,000)$ & 19.25 & 34.44 & 4.92 & 4.92 & $178.9 \%$ \\
\hline \multirow[t]{2}{*}{ Leather } & Thousand skins & 17148.00 & 25200.00 & 3600.00 & 3600.00 & $147.0 \%$ \\
\hline & $(\$ 000,000)$ & 39.76 & 105.84 & 15.12 & 15.12 & $266.2 \%$ \\
\hline \multirow[t]{2}{*}{ Karakul } & Thousand pelts & 8570.70 & 8400.00 & 1125.00 & 1275.00 & $98.0 \%$ \\
\hline & $(\$ 000,000)$ & 98.51 & 121.80 & 16.31 & 18.49 & $123.6 \%$ \\
\hline \multirow[t]{2}{*}{ Wool } & Thousand tons & 34.20 & 33.60 & 4.80 & 4.80 & $98.2 \%$ \\
\hline & $(\$ 000,000)$ & 46.50 & 47.68 & 6.81 & 6.81 & $102.5 \%$ \\
\hline \multirow[t]{2}{*}{ Ginned cotton } & Thousand tons & 122.10 & 394.00 & 40.10 & 76.30 & $322.7 \%$ \\
\hline & $(\$ 000,000)$ & 109.82 & 512.20 & 52.13 & 99.19 & $466.4 \%$ \\
\hline \multirow[t]{2}{*}{ Medical herbs } & Thousand tons & 63.60 & 159.98 & 17.93 & 28.43 & $251.5 \%$ \\
\hline & $(\$ 000,000)$ & 23.48 & 53.91 & 6.04 & 9.59 & $229.6 \%$ \\
\hline \multirow[t]{2}{*}{ Carpet \& rugs } & Thous. sq. meters & 3279.00 & 4350.00 & 560.00 & 680.00 & $132.7 \%$ \\
\hline & $(\$ 000,000)$ & 83.34 & 166.61 & 21.45 & 26.04 & $199.9 \%$ \\
\hline \multirow[t]{2}{*}{ Fur coats } & Thousand pieces & 1040.80 & 595.00 & 70.00 & 100.00 & $57.2 \%$ \\
\hline & $(\$ 000,000)$ & 12.47 & 6.55 & 0.77 & 1.10 & $52.5 \%$ \\
\hline \multirow[t]{2}{*}{ Meat } & Thousand tons & 0.00 & 20.50 & 0.00 & 6.00 & \\
\hline & $(\$ 000,000)$ & 0.00 & 41.00 & 0.00 & 12.00 & \\
\hline \multirow[t]{2}{*}{ Olive } & Thousand tons & 0.29 & 20.14 & 0.50 & 5.90 & $6944.8 \%$ \\
\hline & $(\$ 000,000)$ & 0.24 & 16.11 & 0.40 & 4.72 & $6713.3 \%$ \\
\hline \multirow[t]{2}{*}{ Cement } & Thousand tons & 83.00 & 1367.00 & 0.00 & 383.00 & $1647.0 \%$ \\
\hline & $(\$ 000,000)$ & 3.12 & 68.35 & 0.00 & 19.15 & $2188.6 \%$ \\
\hline \multirow[t]{2}{*}{ Textiles } & Thousand meters & 6358.00 & 0.00 & 0.00 & 0.00 & \\
\hline & $(\$ 000,000)$ & 1.41 & 0.00 & 0.00 & 0.00 & \\
\hline Other commodities & $(\$ 000,000)$ & 42.77 & 52.50 & 6.00 & 9.00 & $122.8 \%$ \\
\hline Total exports & & 1043.78 & 2184.32 & 248.72 & 393.49 & $209.3 \%$ \\
\hline
\end{tabular}

Source: GOA First Seven - Year Economic and Social Development Plan, 1976-83, Ministry of Planning, Kabul

1976; cited from "Afghanistan: The Journey to Economic Development", Vol. II, March 1978, World Bank. 
Table A V-17. Major Exports Quantity Indices

$(1968 / 69=100)$

\begin{tabular}{|c|c|c|c|c|c|c|c|c|c|}
\hline Yr. ended March 20 & $1968 / 69$ & $1969 / 70$ & $1970 / 71$ & $1971 / 72$ & $1972 / 73$ & $1973 / 74$ & $1974 / 75$ & 1975/76 [a] & $1976 / 77$ [a] \\
\hline 1. Fresh fruits & 100 & 118 & 108 & 116 & 106 & 141 & 225 & 182 & 167 \\
\hline 2. Dry fruits & 100 & 120 & 144 & 117 & 173 & 178 & 208 & 183 & 204 \\
\hline 3. Karakul & 100 & 142 & 117 & 142 & 100 & 75 & 100 & 67 & 92 \\
\hline 4. Cotton & 100 & 101 & 179 & 279 & 205 & 129 & 312 & 486 & 517 \\
\hline 5. Carpets, Rugs & 100 & 128 & 113 & 164 & 183 & 171 & 171 & 139 & 218 \\
\hline 6. Natural gas & 100 & 138 & 163 & 163 & 175 & 175 & 181 & 175 & 138 \\
\hline \multicolumn{10}{|l|}{ Weighted Indices } \\
\hline Base year & 100 & 127 & 162 & 152 & 156 & 148 & 189 & 184 & 197 \\
\hline Last year & 100 & 119 & 147 & 177 & 170 & 148 & 242 & 257 & 278 \\
\hline Fisher & 100 & 123 & 154 & 164 & 163 & 148 & 214 & 218 & 234 \\
\hline
\end{tabular}

[a] Preliminary

Source: World Bank mission estimates; cited from "Afghanistan: The Journey to Economic Development", Vol. II, March 1978, World Bank. 


\begin{tabular}{|c|c|c|c|c|c|c|c|c|c|c|c|c|c|c|c|c|c|c|c|c|c|c|c|c|}
\hline & $\begin{array}{l}1965 \\
\text { Exp. }\end{array}$ & Imp. & $\begin{array}{l}1966 \\
\text { Exp. }\end{array}$ & Imp. & $\begin{array}{l}1967 \\
\text { Exp. }\end{array}$ & Imp. & $\begin{array}{l}1968 \\
\text { Exp. }\end{array}$ & Imp. & $\begin{array}{l}1969 \\
\text { Exp. }\end{array}$ & Imp. & $\begin{array}{l}1970 \\
\text { Exp. }\end{array}$ & Imp. & $\begin{array}{l}1971 \\
\text { Exp. }\end{array}$ & Imp. & $\begin{array}{l}1972 \\
\text { Exp. }\end{array}$ & Imp. & $\begin{array}{l}1973 \\
\text { Exp. }\end{array}$ & Imp. & $\begin{array}{l}1974 \\
\text { Exp. }\end{array}$ & Imp. & $\begin{array}{l}1975 \\
\text { Exp. }\end{array}$ & Imp. & $\begin{array}{l}1976 \\
\text { Exp. }\end{array}$ & Imp. \\
\hline U.S.S.R. & 17.5 & 61.0 & 23.7 & -- & 22.1 & 66.5 & 26.6 & 47.8 & 29.9 & 41.7 & 32.5 & 37.5 & 38.6 & 40.3 & 36.1 & -- & 48.8 & 18.5 & 92.4 & 73.3 & 86.4 & 83.4 & 83.7 & 84.7 \\
\hline Japan & -- & 8.0 & -- & 11.9 & 0.2 & 10.6 & 0.1 & 12.0 & -- & 14.6 & 0.2 & 19.0 & 0.4 & 19.6 & 0.1 & 36.5 & 0.7 & 32.5 & 1.0 & 59.7 & 0.5 & 67.6 & 3.1 & 70.9 \\
\hline United Kingdom & 12.3 & 4.7 & 11.1 & 3.9 & 10.7 & 3.6 & 7.4 & 3.2 & 12.9 & 5.2 & 13.6 & 5.3 & 19.8 & 10.6 & 19.9 & 6.5 & 22.6 & 6.6 & 22.8 & 8.4 & 15.1 & 12.7 & 55.2 & 11.7 \\
\hline Fed. Rep. of Germary & 5.5 & 17.3 & 3.7 & 10.2 & 0.6 & 11.2 & 1.3 & 11.3 & 1.6 & 7.0 & 4.3 & 7.4 & 8.7 & 11.0 & 7.7 & 12.9 & 7.0 & 8.7 & 5.7 & 10.6 & 22.3 & 43.5 & 18.2 & 22.9 \\
\hline Pakistan & 9.6 & 4.0 & 5.5 & 15.5 & 5.5 & 3.3 & 5.7 & 3.1 & 6.3 & 3.6 & 6.1 & 3.3 & 5.2 & 3.6 & 5.3 & 7.2 & 22.4 & 5.4 & 26.0 & 10.8 & 29.1 & 5 & 33.4 & 8.5 \\
\hline India & 4.9 & 5.0 & 9.1 & 12.4 & 10.8 & 6.3 & 15.8 & 11.4 & 15.1 & 11.4 & 13.4 & 12.7 & 4.8 & 11.4 & 30.4 & 13.8 & 27.5 & 19.4 & 37.5 & 24.7 & 26.9 & 41.4 & 25.9 & 24.6 \\
\hline United States & 11.0 & 20.0 & 5.5 & 15.1 & 5.6 & 17.7 & 4.6 & 10.5 & 2.5 & 7.0 & 2.5 & 3.5 & 2.1 & 32.3 & 2.5 & 19.3 & 2.1 & 6.1 & 5.1 & 10.5 & 9.4 & 22.8 & 11.7 & 19.8 \\
\hline Singapore & -- & -- & -- & -- & 0.1 & 0.8 & -- & -- & -- & 0.2 & 0.1 & 0.5 & 0.1 & 0.8 & 0.1 & 1.8 & 0.6 & 1.5 & 0.6 & 3.4 & 0.2 & 4.6 & 0.2 & 3.9 \\
\hline China & 0.1 & -- & 0.4 & -- & 0.4 & 5.6 & -- & -- & 0.5 & 10.5 & -- & -- & -- & -- & 1.1 & -- & 3.5 & 6.4 & 2.9 & 3.0 & 2.9 & 2.8 & 4.3 & 5.0 \\
\hline Republic of Korea & -- & -- & -- & -- & -- & $-\overline{-}$ & -- & -- & -- & -- & $-\overline{-}$ & -- & -- & -- & -- & -- & -- & -- & & & -- & -- & -- & -- \\
\hline Hong Kong & -- & -- & -- & 0.6 & 0.0 & 0.7 & -- & -- & -- & 0.9 & 0.0 & 0.8 & 0.0 & 0.6 & 0.0 & 0.9 & 0.0 & 1.3 & 0.0 & 2.7 & 0.0 & 3.4 & 0.0 & 6.7 \\
\hline Switzerland & -- & -- & -- & 2.1 & 3.8 & 1.3 & -- & -- & 4.6 & -- & 3.9 & 1.6 & 4.6 & 1.9 & 4.8 & 2.1 & 5.9 & 2.9 & 1. & 3.1 & 5.9 & 2.1 & 6.0 & 5.1 \\
\hline France & -- & -- & -- & 0.9 & 0.0 & 0.9 & -- & -- & -- & 1.2 & 0.5 & 1.4 & 0.8 & 1.3 & 2.7 & 5.8 & 1.3 & 1.6 & 1.3 & 2.3 & 1.1 & 5.4 & 7.8 & 5.9 \\
\hline Iran & -- & -- & 0.5 & 1.2 & 0.7 & 2.9 & -- & $\ldots$ & 1.0 & 3.6 & 0.6 & 2.8 & 0.3 & 5.3 & 0.8 & -- & 2.1 & 4.7 & 5.2 & 13.1 & 5.1 & 14.3 & 5.2 & 19.1 \\
\hline Netherlands & -. & -- & -- & 1.5 & 0.1 & 1.4 & -- & -- & 10 & 18 & 1.4 & 1.5 & 3.5 & 1.6 & 0.7 & 2.4 & 1.0 & 1.5 & 0.5 & 5.0 & 1.5 & 5.1 & 8.1 & 3.7 \\
\hline & -- & -- & 1.6 & 1.3 & 1.4 & 1.3 & -- & -- & 1.2 & 1.7 & 1.0 & 0.8 & 1.7 & 1.2 & 4.0 & 2.0 & 3.7 & 0.8 & 4.5 & 1.1 & 3.0 & 1.4 & 6.7 & 1.9 \\
\hline Czechoslovakia & 1.2 & 3.9 & 2.1 & - & 2.3 & 0.8 & 1.5 & 0.8 & 1.6 & 0.5 & 0.6 & 0.8 & 1.3 & 0.5 & 2.8 & $\ldots$ & 1.5 & 0.7 & 3.1 & 0.8 & 2.1 & 2.9 & 5.3 & 7.4 \\
\hline Saudi Arabia & - & - & - & -- & -- & -- & -- & -- & -- & -- & 0.0 & -- & 0.1 & 0.0 & 0.1 & 0.1 & 0.1 & 0.0 & 0.4 & 1.9 & 1.3 & 3.3 & 6.5 & 2.7 \\
\hline Belgium-Luxembourg & -- & -- & -- & 0.1 & -- & -- & -- & -- & -- & -- & 0.1 & 0.3 & 0.0 & 0.2 & 0.1 & 0.7 & 0.6 & 0.4 & 0.1 & 0.5 & 0.1 & 1.2 & 0.2 & 1.0 \\
\hline Thailland & -- & -- & -- & -- & -- & -- & -- & -- & -- & -- & - & -- & -- & -- & - & 0.6 & 0.0 & 0.2 & - & 0.7 & -- & 0.6 & - & 0.6 \\
\hline Australia & - & -- & 0.4 & -- & -- & -- & -- & -- & -- & 2.0 & 0.0 & 0.1 & 0.1 & 0.1 & 0.1 & -- & 0.0 & 0.2 & 0.1 & 0.1 & 0.1 & 0.1 & 0.4 & 0.0 \\
\hline Lebanon & -- & -- & -- & 0.3 & 1.9 & 0.6 & -- & -- & 1.8 & 0.2 & 1.5 & 0.5 & 5.4 & 0.6 & 3.6 & 0.3 & 3.9 & 1.0 & 5.8 & 0.7 & 3.4 & -- & 0.6 & 0.0 \\
\hline Norway & -- & -- & -- & 0.1 & -- & -- & -- & -- & -- & -- & 0.2 & 0.5 & 0.1 & 0.4 & 0.0 & 0.3 & 0.1 & 0.3 & 0.2 & 0.1 & 0.1 & 0.3 & 0.1 & 0.5 \\
\hline Denmark & -- & -- & -- & 0.3 & -- & -- & -- & -- & -- & -- & 0.1 & 0.2 & 0.2 & 0.2 & 0.4 & 1.2 & 1.2 & 0.2 & 0.9 & 0.5 & 0.7 & 3.8 & 0.8 & 0.6 \\
\hline German Dem. Rep. & -- & -- & -- & -- & -- & -- & -- & -- & -- & -- & -- & -- & -- & -- & -- & -- & -- & -- & -- & -- & 0.0 & 0.0 & -- & -- \\
\hline Austria & -- & -- & -- & 0.3 & -- & -- & -- & -- & -- & -- & 0.0 & 0.1 & 0.3 & 0.1 & 0.0 & 0.8 & 0.0 & 0.1 & 0.2 & 0.4 & 0.1 & 0.7 & 0.1 & 0.5 \\
\hline Syria & -- & & 0.1 & & -- & & -- & & -- & & -- & & -- & & -- & & 0.1 & & 0.1 & & 0.2 & & 8.9 & \\
\hline Iceland & -- & -- & -- & -- & -- & -- & -- & -- & -- & -- & 0.1 & 0.2 & 0.1 & 0.3 & 0.3 & -- & 0.6 & 0.3 & 9.3 & 0.0 & 0.5 & 1.7 & 0.8 & 1.0 \\
\hline Sri Lanka & -- & -- & -- & -- & -- & -- & -- & -- & -- & -- & -- & -- & -- & -- & -- & -- & 0.0 & 0.4 & -- & 0.6 & -- & 0.3 & -- & 1.7 \\
\hline Bangladesh & -- & -- & -- & -- & -- & -- & -- & -- & -- & -- & -- & -- & -- & -- & -- & 0.1 & -- & -- & -- & -- & -- & -- & -- & -- \\
\hline Malaysia & -- & -- & -- & -- & -- & -- & -- & -- & -- & -- & -- & -- & -- & -- & -- & -- & -- & -- & -- & 0.0 & 0.6 & 0.0 & 0.0 & 1.1 \\
\hline Ireland & -- & -- & -- & -- & -- & -- & -- & -- & -- & -- & -- & -- & -- & -- & -- & -- & -- & -- & -- & -- & -- & -- & -- & -- \\
\hline Turk & -- & -- & -- & $-\overline{0}$ & -- & -- & -- & -- & -- & -- & -- & -- & -- & -- & -- & -- & -- & -- & -- & -- & -- & -- & -- & 0.0 \\
\hline Swe & -- & -- & -- & 0.2 & -- & -- & -- & -- & -- & -- & -- & -- & -- & -- & -- & 0.4 & -- & -- & -- & -- & $-\bar{x}$ & & & -- \\
\hline Spa & -- & -- & -- & 0.9 & -- & -- & -- & -- & -- & -- & -- & -- & -- & -- & -- & 0.1 & $-\overline{-}$ & -- & -- & $\overline{-}$ & 0.1 & 0.1 & 0.2 & 0.1 \\
\hline Finla & -- & -- & -- & -- & -- & -- & -- & -- & -- & -- & -- & -- & -- & -- & -- & -- & 0.2 & $\overline{0}$ & 0.1 & 0.0 & 1.0 & -- & 0.7 & 0.2 \\
\hline Yugo & - & -- & -- & -- & -- & -- & -- & -- & -- & -- & -- & -- & $-\overline{0}$ & -- & $-\overline{-}$ & -- & $-\overline{-}$ & 0.0 & $\overline{-}$ & 0.2 & 0.5 & 0.1 & $-\overline{0}$ & 0.1 \\
\hline Poland & -- & -- & 0.1 & -- & -- & -- & $=-$ & -- & 0.1 & 0.0 & 0.4 & 0.1 & 0.0 & 0.0 & 0.3 & $=-$ & 0.0 & 0.1 & 1.7 & 0.2 & $=-$ & 0.3 & 0.0 & 0.3 \\
\hline Hungary & -- & -- & -- & -- & -- & 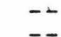 & $=$ & $=-$ & $=-$ & -- & $\overline{01}$ & $-\overline{1}$ & -- & $\overline{12}$ & -- & $\overline{0}$ & $-\overline{0}$ & $\overline{0}$ & $-\overline{-}$ & $=-$ & $\overline{0}$ & -- & 0.2 & 0.1 \\
\hline Canada & -- & $=-$ & -- & $=-$ & -- & $\overline{--}$ & $=-$ & $=-$ & -- & -- & $\begin{array}{l}0.1 \\
0.0\end{array}$ & 1.2 & 0.1 & $\begin{array}{l}1.2 \\
0.3\end{array}$ & 0.1 & $\begin{array}{l}0.1 \\
0.4\end{array}$ & $\begin{array}{l}0.0 \\
0.4\end{array}$ & $\begin{array}{l}0.0 \\
--\end{array}$ & $\begin{array}{l}0.1 \\
10\end{array}$ & 0.0 & 0.2 & 0.1 & 0.5 & 0.0 \\
\hline $\begin{array}{l}\text { Iraq } \\
\text { Kuwait }\end{array}$ & - & -- & -- & - & - & - & -- & - & -- & - & -- & -- & $\begin{array}{l}0.1 \\
--\end{array}$ &.- & 0.2 & $\begin{array}{l}0.4 \\
--\end{array}$ & 0.0 & 0.2 & 0.0 & 0.5 & 0.3 & $\begin{array}{l}0.9 \\
1.1\end{array}$ & 0.9 & 0.2 \\
\hline $\begin{array}{l}\text { Luwan } \\
\text { Cameroon }\end{array}$ & -- & & -- & & -- & & -. & & -- & & -- & & -- & & -- & & -- & & -- & & -- & & -- & \\
\hline
\end{tabular}




\begin{tabular}{|c|c|c|c|c|c|c|c|c|c|c|c|c|c|c|c|c|c|c|c|c|c|c|c|c|}
\hline & $\begin{array}{l}1965 \\
\text { Exp. }\end{array}$ & Imp. & $\begin{array}{l}1966 \\
\text { Exp. }\end{array}$ & Imp. & $\begin{array}{l}1967 \\
\text { Exp. }\end{array}$ & Imp. & $\begin{array}{l}1968 \\
\text { Exp. }\end{array}$ & Imp. & $\begin{array}{l}1969 \\
\text { Exp. }\end{array}$ & Imp. & $\begin{array}{l}1970 \\
\text { Exp. }\end{array}$ & Imp. & $\begin{array}{l}1971 \\
\text { Exp. }\end{array}$ & Imp. & $\begin{array}{l}1972 \\
\text { Exp. }\end{array}$ & Imp. & $\begin{array}{l}1973 \\
\text { Exp. }\end{array}$ & Imp. & $\begin{array}{l}1974 \\
\text { Exp. }\end{array}$ & Imp. & $\begin{array}{l}1975 \\
\text { Exp. }\end{array}$ & Imp. & $\begin{array}{l}1978 \\
\text { Exp. }\end{array}$ & Imp. \\
\hline Indonesia & -- & -- & -- & -- & -- & -- & -- & -- & -- & -- & -- & -- & -- & -- & -- & -- & -- & -- & -- & -- & 0.0 & 0.0 & -- & - \\
\hline Bulgaria & -- & -- & -- & -- & -- & -- & -- & -- & -- & -- & -- & -- & -- & -- & -- & -- & 0.2 & -- & 0.4 & 0.1 & 0.1 & 0.0 & 0.8 & 0.2 \\
\hline Egypt & -- & -- & 0.1 & -- & -- & -- & -- & -- & -- & -- & -- & -- & -- & -- & -- & -- & -- & 0.0 & -- & 0.0 & -- & -- & -- & -- \\
\hline $\begin{array}{l}\text { United Arab Emirates } \\
\text { Jordan }\end{array}$ & $=-$ & -- & $=-$ & -- & $=-$ & -- & $=-$ & -- & $=-$ & -- & -- & -- & $=-$ & -- & -- & -- & -- & -- & $=-$ & -- & -- & -- & -- & -- \\
\hline $\begin{array}{l}\text { Jordan } \\
\text { Portugal }\end{array}$ & - & -- & - & -- & - & -- & - & -- & - & -- & -- & -- & - & -- & - & 0.0 & - & -- & - & -- & $=-$ & -- & -- & -- \\
\hline $\begin{array}{l}\text { Romania } \\
\text { Maurttania }\end{array}$ & -- & -- & $\ldots$ & -- & -. & -- & -- & -- & _- & -- & _- & -- & $\ldots$ & -- & -- & -- & $\ldots$ & 0.1 & $\ldots$ & -- & _- & 0.2 & $\ldots$ & 0.3 \\
\hline Greece & - & -- & -- & -- & $\ldots$ & -- & - & -- & -- & -- & -- & -- & -- & -- & - & -- & - & -- & - & -- & -- & -- & -- & -- \\
\hline Montserrat & & -- & & -- & & -- & & -- & & -- & & -- & & -- & & -- & 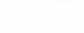 & -- & 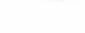 & -- & 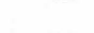 & -- & 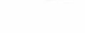 & -- \\
\hline New Zealand & -- & -- & -- & -- & -- & -- & -- & -- & -- & -- & -- & -- & -- & -- & -- & 0.0 & -- & -- & -- & -- & 0.0 & 0.0 & -- & -- \\
\hline $\begin{array}{l}\text { Cyprus } \\
\text { Belize }\end{array}$ & $=-$ & -- & $=-$ & -- & $=-$ & -- & $=-$ & -- & $=-$ & -- & $=-$ & - & $=-$ & -- & $=-$ & -- & $=-$ & -- & $=-$ & -- & $=-$ & -- & $=-$ & -- \\
\hline Burkina Faso & -- & & -- & & -- & & -- & & -- & & -- & & -- & & -- & & -- & & - & & -- & & 0.3 & \\
\hline South Atrica & -- & & -- & & -- & & -- & & -- & & -- & & -- & & -- & & 0.0 & & 0.2 & & -- & & 0.1 & \\
\hline $\begin{array}{l}\text { Congo } \\
\text { Brazil }\end{array}$ & -- & $\ldots$ & -- & & -- & & -- & & -- & & -- & & -- & & -- & & -- & & -- & & -- & & -- & \\
\hline $\begin{array}{l}\text { Brazil } \\
\text { Philippines }\end{array}$ & & $\overline{--}$ & & $=-$ & & $=-$ & & $=-$ & & $=-$ & & $=-$ & & $=-$ & & 0.1 & & $=-$ & & $=-$ & & $=-$ & & $=$ \\
\hline Malta & -- & & -- & & -- & & -- & & -- & & -- & & -- & & -- & 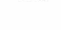 & -- & 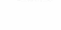 & -- & . & -- & . & -- & - \\
\hline Tunisia & -- & -- & -- & -- & -- & -- & -- & -- & -- & -- & -- & -- & -- & -- & -- & -- & -- & -- & -- & -- & -- & -- & -- & -- \\
\hline Kenya & -- & & -- & 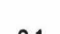 & -- & & -- & & -- & & -- & & -- & & -- & $\ldots$ & -- & & -- & & -- & & -- & \\
\hline $\begin{array}{l}\text { Souvth Yemen } \\
\text { Greenland }\end{array}$ & -- & - & -- & 0.1 & -- & -- & -- & -- & -- & -- & -- & -- & -- & -- & -- & -- & -- & -- & -- & -- & -- & - & -- & -- \\
\hline Bermuda & -- & & -- & & -- & & -- & & -- & & -- & & -- & & -- & & -- & & -- & & -- & & -- & \\
\hline inezuela & -- & & -- & & -- & & -- & & -- & & -- & & -- & & -- & & -- & & -- & & -- & & -- & \\
\hline Iamaica & -- & & -- & & -- & & -- & & -- & & -- & & -- & & -- & & -- & & -- & & -- & & -- & \\
\hline Burunc & -- & & -- & & -- & & -- & & -- & & -- & & -- & & -- & & -- & & -- & & -- & & -- & \\
\hline Trinidad \& Tobago & -- & -- & -- & -- & -- & -- & -- & -- & -- & -- & -- & -- & -- & -- & -- & -- & $=-$ & $=-$ & -- & $=-$ & -- & -- & -- & $=-$ \\
\hline $\begin{array}{l}\text { Macau } \\
\text { Mozambique }\end{array}$ & - & - & & $=$ & . & - & . & - & - & -- & - & $=$ & - & -- & & 0.0 & & -- & - & -- & - & $=$ & - & - \\
\hline Somalia & -- & -- & -- & -- & -- & -- & -- & -- & -- & -- & -- & -- & -- & -- & -- & 0.0 & -- & -- & -- & -- & -- & -- & -- & -- \\
\hline Peru & -- & & -- & & -- & & -- & & -- & & -- & & -- & & -- & & -- & & -- & & -- & & -- & \\
\hline \multicolumn{25}{|l|}{ Source: IMF } \\
\hline & ERR & & ERR & & ER & ERT & ERR & ERF & ERR & ER & ERR & ERF & & EF & ERR & ERR & EF & ERI & ERR & ERR & ERR & ERR & ERR & ERR \\
\hline East N. Africa & & & & & & & & & & & & & & & & & & & & & & & & 8.8 \\
\hline & & 50. & 18 & 54 & & 55 & 21 & 45 & 27 & 53 & 27 & & & & & & & & 69 & 121 & & 192 & 79.8 & 161.8 \\
\hline & & & & 24. & & & & & 23 & & & & & & & 24.7 & & & & & & & 132.6 & 62.1 \\
\hline & 28.8 & 50.0 & 22.3 & -- & 22.3 & 48 & 13.5 & 37. & 23.7 & 40 & 28. & & 42. & & 43.4 & -- & 47. & & 53.1 & 102 & 61.6 & 168 & 120.6 & 145.7 \\
\hline Developing countri & 14.6 & 9.0 & 15.8 & -- & 19.4 & 20.2 & 21.5 & 14.5 & 24.7 & 30.5 & 22.1 & 20.7 & 16.0 & 22.6 & 41.9 & -- & 60.7 & 40.7 & 81.4 & 62.4 & 71.7 & 80.9 & 86.7 & 76.1 \\
\hline Hemisphere & & & & -- & & & & & & & & & & & & & & & & & & & & \\
\hline necon & 19.3 & 66 & 27.0 & & 24. & 67.3 & 28.1 & 48. & 31.5 & 42 & 33 & 38 & 39.9 & 40 & 39.0 & & 50.5 & 19. & 95.9 & 74. & 88.7 & 86.3 & 89.8 & 92.3 \\
\hline & 23 & 12.6 & 20 & 21.6 & 19 & & & 7. & & 14 & 22 & 11 & 28. & 17 & 35 & 21.7 & & 15. & & & 50 & & 117. & 36.0 \\
\hline Oil exporting nations & & - & & -- & 0 & 2 & & & & 3 & & & 0 & 5 & & -- & & 4 & 6.6 & 15.5 & 7.5 & 19.7 & 12.7 & 23.1 \\
\hline Non-oil & 14.6 & 9.0 & 15.3 & -- & 18. & 17.3 & 21.5 & 14.5 & 23 & 27. & 21. & 17. & 15 & 17.0 & 40.8 & $-\overline{-}$ & 58.1 & 35.8 & 74.8 & 46.9 & 64.2 & 61.2 & 74.0 & 53.0 \\
\hline World total & 0.0 & 0.0 & 0.0 & 0.0 & 0.0 & 0.0 & 0.0 & 0.0 & 0.0 & 0.0 & 0.0 & 0.0 & 0.0 & 0.0 & 0.0 & 0.0 & 0.0 & 0.0 & 0.0 & 0.0 & 0.0 & 0.0 & 0.0 & 0.0 \\
\hline
\end{tabular}

Source: IMF

alt e $\quad /$ re $\sim$ \{right\} \{right\} (right\} 
llions of U.S. dollars the Value of Alghanistan's Exp orts and Imporis
(millions of U.S. dollars)

\begin{tabular}{|c|c|c|c|c|c|c|c|c|c|c|c|c|c|c|c|c|c|c|c|c|c|c|c|}
\hline $\begin{array}{c}1977 \\
\text { Exp. }\end{array}$ & Imp. & $\begin{array}{c}1978 \\
\text { Exp. }\end{array}$ & Imp. & $\begin{array}{l}1979 \\
\text { Exp. }\end{array}$ & Imp. & $\begin{array}{c}1980 \\
\text { Exp. }\end{array}$ & Imp. & $\begin{array}{l}1981 \\
\text { Exp. }\end{array}$ & Imp. & $\begin{array}{c}1982 \\
\text { Exp. }\end{array}$ & Imp. & $\begin{array}{l}1983 \\
\text { Exp. }\end{array}$ & Imp. & $\begin{array}{l}1984 \\
\text { Exp. }\end{array}$ & Imp. & $\begin{array}{l}1985 \\
\text { Exp. }\end{array}$ & Imp. & $\begin{array}{l}1986 \\
\text { Exp. }\end{array}$ & Imp. & $\begin{array}{c}1987 \\
\text { Exp. }\end{array}$ & Imp. & $\begin{array}{l}1988 \\
\text { Exp. }\end{array}$ & Imp. \\
\hline 116.0 & 111.6 & 112.8 & 240.0 & 255.1 & 370.0 & 417.9 & 290.5 & 412.6 & 365.0 & -- & -- & -- & -- & -- & -- & -- & - & -- & - & -- & -- & -- & -- \\
\hline 4.8 & 101.5 & & 102.8 & & 80.8 & 0.8 & 98.2 & 0.8 & 78.7 & 0.8 & 106.1 & 0.2 & 132.7 & 1.0 & 123.0 & 2.3 & 108.4 & 1.0 & 129.9 & 2.5 & 156.4 & -- & -- \\
\hline 37.1 & 15.6 & 30.2 & 21.7 & 32.6 & 11.1 & 51.8 & 10.3 & 36.3 & 13.4 & 32.6 & 17.6 & 27.3 & 17.3 & 24.4 & 17.7 & 60.3 & 19.6 & 15.9 & 18.5 & 16.9 & 19.2 & 18.4 & 23.7 \\
\hline 17.4 & 31.9 & 22.5 & 22.2 & 32.5 & 22.6 & 51.5 & 17.0 & 41.8 & 16.8 & 32.6 & 26.1 & 36.7 & 23.5 & 30.2 & 25.1 & 22.4 & 23.5 & 28.3 & 24.9 & 35.7 & 32.3 & -- & -- \\
\hline 36.5 & 12.5 & & 16.9 & 52.9 & 13.7 & 52.1 & 14.9 & 61.2 & 11.7 & 56.3 & 18.9 & 21.2 & 17.3 & 21.8 & 21.3 & 16.5 & 16.7 & 14.5 & 29.1 & 10.2 & 27.8 & -- & -- \\
\hline 23.9 & 24.5 & & 37.1 & 42.1 & 22.3 & 54.7 & 20.6 & 43.2 & 17.0 & -- & -- & -- & $=-$ & -- & -- & -- & -- & -- & -- & -- & -- & -- & -- \\
\hline 14.9 & 16.8 & 11.3 & 8.9 & 4.1 & 21.0 & 4.9 & 14.2 & 9.7 & 7.2 & 11.3 & 11.0 & 8.0 & 5.6 & 12.6 & 8.1 & 6.5 & 3.9 & 4.9 & 8.4 & 5.5 & 8.7 & -- & -- \\
\hline 0.2 & 6.1 & -- & -- & -- & -- & -- & -- & -- & -- & 0.2 & 36.4 & 0.1 & 34.3 & 0.6 & 32.1 & 0.0 & 56.4 & 0.1 & 57.8 & 0.1 & 78.9 & -- & -- \\
\hline 6.9 & 5.6 & -- & -- & -- & -- & 0.2 & 8.2 & -- & 1.1 & 3.0 & 2.4 & 0.2 & 8.1 & 0.1 & 8.6 & 0.2 & 19.0 & 0.2 & 75.8 & 0.1 & 76.2 & -- & -- \\
\hline-- & -- & -- & -- & -- & -- & -- & -- & -- & -- & $-\overline{0}$ & 10.6 & 0.0 & 20.4 & -- & 16.7 & $-\overline{-}$ & 31.7 & 0.0 & 71.5 & -- & 80.2 & -- & -- \\
\hline 0.2 & 9.4 & -- & -- & $-\overline{-}$ & -- & 0.0 & 18.6 & 0.0 & 27.4 & 0.0 & 21.7 & 0.1 & 21.0 & 0.1 & 23.2 & 0.0 & 21.7 & 0.1 & 33.6 & & 26.9 & -- & -- \\
\hline 7.8 & 4.2 & 8.9 & -- & 9.8 & -- & -- & -- & -- & -- & 11.1 & 3.3 & 7.3 & 26.4 & 6.3 & & 6.1 & 2.0 & 7.1 & & 9.4 & 22.4 & 7.4 & 2.9 \\
\hline 1.8 & 7.1 & -- & -- & -- & -- & $-\overline{-}$ & -- & $-\overline{-}$ & $-\overline{-}$ & 4.9 & 21.0 & 3.5 & 12.4 & 3.2 & 10.2 & 3.2 & 13.2 & 3.5 & 14.2 & 3.8 & 15.0 & 3.8 & 12.9 \\
\hline 2.5 & 66.8 & -- & -- & -- & -- & 0.4 & 0.5 & 0.2 & 0.0 & $-\overline{8}$ & $-\overline{-}$ & -- & -- & -- & $\overline{-}$ & $\overline{-1}$ & $\overline{-}$ & $\overline{-}$ & -- & $-\overline{2}$ & -- & -- & -- \\
\hline 1.9 & $\begin{array}{l}4.6 \\
3.6\end{array}$ & -- & -- & -- & -- & -- & -- & -- & -- & $\begin{array}{l}1.8 \\
3.8\end{array}$ & 2.5 & 1.8 & 3.9 & 16.9 & 16.4 & 3.6 & 3.0 & 2.0 & $\begin{array}{r}3.9 \\
5.9\end{array}$ & 5.3 & 8.4 & -- & -- \\
\hline $\begin{array}{l}5.6 \\
5.3\end{array}$ & $\begin{array}{r}3.6 \\
151\end{array}$ & $\overline{48}$ & $\overline{8}$ & $\overline{60}$ & $\overline{0.5}$ & $=-$ & $\overline{10}$ & $=-$ & $\overline{24}$ & $\begin{array}{l}3.8 \\
--\end{array}$ & $\begin{array}{l}4.0 \\
--\end{array}$ & $\begin{array}{l}4.6 \\
-\end{array}$ & $\begin{array}{r}4.9 \\
-\end{array}$ & 6.2 & 4.4 & $\begin{array}{l}5.8 \\
--\end{array}$ & 5.1 & 6.9 & 5.0 & 8.0 & $\begin{array}{l}4.4 \\
-\end{array}$ & $=-$ & $=-$ \\
\hline $\begin{array}{r}5.3 \\
14.3\end{array}$ & $\begin{array}{r}15.1 \\
0.0\end{array}$ & 4.8 & $\begin{array}{l}0.8 \\
--\end{array}$ & $\begin{array}{l}6.0 \\
--\end{array}$ & $\begin{array}{l}9.5 \\
--\end{array}$ & $21 . \overline{2}$ & $\begin{array}{l}1.0 \\
0.0\end{array}$ & $19 . \overline{-}$ & $\begin{array}{l}2.4 \\
0.0\end{array}$ & 4.7 & 0.0 & 5.4 & $\overline{-}$ & 3.2 & $\overline{-}$ & 2.1 & $=$ & 1.3 & -- & $=$ & $\overline{-}$ & -- & $\overline{-}$ \\
\hline 0.1 & 1.5 & -- & - & - & -- & -- & -- & -- & - & 4.1 & 2.4 & 3.8 & 1.9 & 2.2 & 0.8 & 0.9 & 1.1 & 4.8 & 2.7 & 12.6 & 2.1 & -- & -- \\
\hline- & 0.7 & -- & -- & -- & -- & -- & -- & -- & -- & 0.0 & 3.8 & 0.0 & 2.9 & 0.5 & 2.9 & 0.0 & 7.4 & -- & 6.0 & -- & 10.1 & -- & -- \\
\hline 0.1 & 0. & -- & -- & -- & -- & -- & -- & -- & -- & 1.3 & 0.0 & 27.8 & 0.0 & 0.9 & 0.3 & 0.3 & 0.0 & 0.3 & -- & 0.2 & -- & -- & -- \\
\hline 0.4 & 0.0 & -- & -- & -- & -- & -- & -- & -- & -- & -- & -- & -- & -- & -- & -- & -- & -- & -- & -- & -- & -- & -- & $\overline{-}$ \\
\hline 0.4 & 0.4 & -- & -- & -- & -- & -- & -- & -- & -- & 1.0 & 0.2 & 0.6 & 0.5 & 0.5 & 9.0 & 0.5 & -- & 0.6 & 2.6 & 0.4 & 4.3 & 0.6 & 2.7 \\
\hline 1.5 & 1.1 & -- & -- & -- & -- & -- & -- & -- & -- & 1.3 & 0.6 & 1.5 & 0.7 & 1.4 & 0.5 & 1.8 & 0.8 & 1.3 & 0.5 & 1.0 & 0.7 & -- & -- \\
\hline & & -- & -- & -- & -- & 14.6 & -- & 12.1 & -- & -- & -- & -- & -- & -- & -- & -- & -- & -- & -- & -- & -- & -- & -- \\
\hline 0.1 & 0.8 & -- & -- & -- & -- & -- & -- & -- & -- & 2.5 & 0.9 & 2.3 & 0.3 & 2.1 & 1.3 & 1.8 & 0.5 & 2.8 & 1.1 & 4.2 & 0.7 & -- & -- \\
\hline 9.0 & & -- & & -- & & -- & & -- & & $\overline{0}$ & & $\overline{-}$ & & $\overline{0}$ & & $\overline{0}$ & & $=-$ & & $\bar{z}$ & & $\overline{-}$ & \\
\hline 0.7 & 1.3 & -- & -- & -- & -- & -- & -- & -- & -- & 0.0 & -- & 0.0 & -- & 0.0 & -- & 0.0 & -- & -- & -- & -- & -- & - & -- \\
\hline-- & 4.7 & -- & -- & -- & -- & -- & -- & -- & -- & 0.0 & 0.6 & $=-$ & 0.6 & -- & 3.0 & 0.0 & 0.7 & -- & 1.2 & -- & -- & -- & -- \\
\hline-- & -- & -- & -- & -- & -- & -- & -- & -- & -- & $\overline{-}$ & 1.2 & 0.1 & 3.9 & 0.4 & 2.9 & -- & 1.4 & -- & 1.5 & -- & 1.1 & -- & -- \\
\hline-- & 1.8 & -- & -- & -- & -- & -- & -- & -- & -- & 0.0 & 3.5 & 0.0 & 0.1 & & 0.0 & & 0.2 & 0.0 & 4.9 & 0.0 & 0.3 & -- & -- \\
\hline 0.4 & -- & -- & -- & -- & -- & -- & -- & -- & -- & 0.8 & 0.7 & 0.7 & 2.5 & & 0.2 & 0. & 1.0 & & 2.2 & 0.1 & 1.0 & -- & -- \\
\hline-- & 4.0 & -- & -- & -- & -- & -- & -- & -- & -- & 0.1 & 0.0 & & 0.1 & & 0.0 & & & & 1.6 & $\overline{-}$ & 3.1 & -- & -- \\
\hline-- & -- & -- & -- & -- & -- & -- & -- & -- & -- & 1.0 & 0. & & 0. & & & & 0.2 & & 0.5 & 1. & 0.4 & -- & -- \\
\hline 0.3 & 0.1 & -- & -- & -- & -- & -- & -- & -- & -- & 0.2 & 0.7 & 0.2 & 0.1 & 0. & 2.1 & & 0.2 & & & & & -- & -- \\
\hline 0.4 & & -- & -- & -- & -- & -- & -- & -- & -- & 0.8 & 0.5 & 0.7 & 0.1 & 0.5 & 0.0 & & 0.3 & 0.5 & 0.3 & 0.3 & 0.4 & -- & -- \\
\hline 0.4 & 0.4 & -- & -- & -- & -- & $\overline{-}$ & -- & -- & $-\bar{z}$ & 0.3 & 2.1 & -- & -- & -- & -- & 0.6 & 0.9 & -- & 2.5 & -- & -- & -- & -- \\
\hline 0.0 & 1. & -- & -- & -- & -- & 0.3 & 0.9 & 1.1 & 0.7 & -- & $=-$ & $-\overline{-}$ & $-\overline{-}$ & -- & $\overline{--}$ & -- & -- & $=-$ & -- & -- & -- & -- & -- \\
\hline 0.3 & 0.4 & -- & $=-$ & -- & -- & -- & $\overline{--}$ & $\overline{-}$ & -- & $\overline{0-}$ & $\overline{0}$ & 0.1 & 4.8 & $\overline{0}$ & $\overline{0-}$ & $\overline{0}$ & $\overline{0}$ & $\overline{0}$ & $\overline{0}$ & $\overline{0}$ & $\overline{0}$ & -- & - \\
\hline 0.1 & 0.3 & -- & -- & -- & -- & -- & -- & -- & -- & 0.4 & 0.2 & 0.2 & 0.1 & $\begin{array}{l}0.1 \\
-\end{array}$ & 0.1 & 0.1 & 0.1 & 0.0 & 0.1 & 0.1 & 0.0 & $=-$ & -- \\
\hline 0.3 & $\overline{10}$ & -- & -- & -- & -- & $\overline{-}$ & $\overline{-}$ & -- & $=-$ & $=-$ & $\overline{-}$ & $=-$ & $=-$ & $\overline{-}$ & $=-$ & $\overline{-}$ & $=-$ & $=-$ & $=-$ & $=-$ & $=-$ & $=-$ & - \\
\hline $\begin{array}{l}1.2 \\
-\ldots\end{array}$ & 1.0 & $\overline{--}$ & -- & -- & -- & -- & -- & $\overline{--}$ & -- & $=-$ & -- & $\overline{-}$ & -- & $4 . \overline{9}$ & - & $\overline{--}$ & -- & $\overline{0.0}$ & -- & $=-$ & -- & $\overline{--}$ & \\
\hline & & & & - & & & & 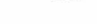 & & & & & & & & & & & & . & & & \\
\hline
\end{tabular}



(millions of U.S. dollars)

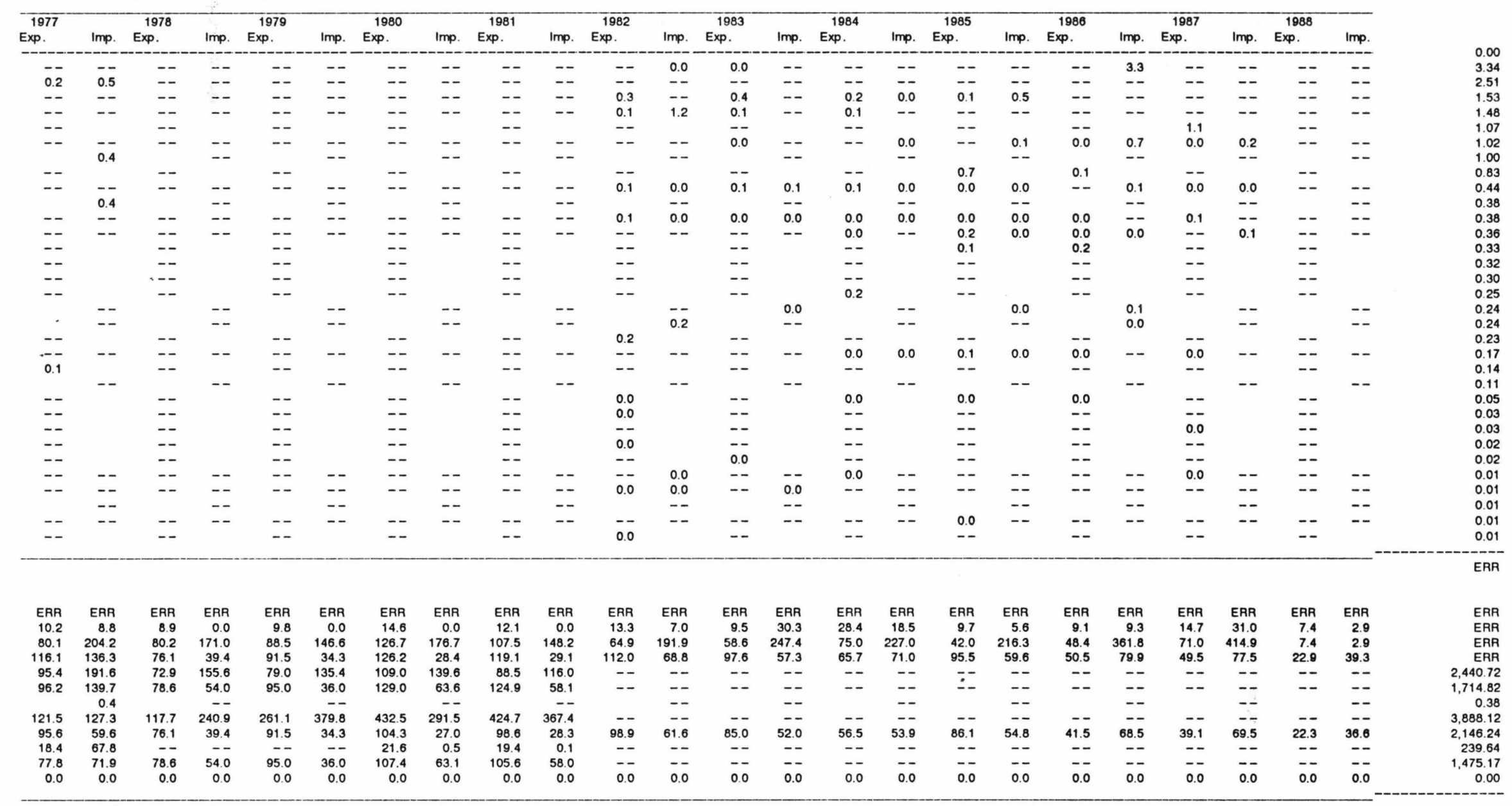


Table A V-19. Merchandise Imports by Category

\begin{tabular}{|c|c|c|c|c|c|}
\hline Item & Units & $1978 / 79$ & $1984 / 85$ & $1985 / 86$ & $1986 / 87$ \\
\hline Consumer Goods & $\$ 000$ & 186,500 & 369,938 & 246,525 & 286,647 \\
\hline Food and Live Animals & $\$ 000$ & 86,348 & 102,192 & 67,145 & 110,639 \\
\hline \multirow[t]{2}{*}{ Wheat } & Thousand Tons & 96.4 & 21.1 & 51.1 & 57.0 \\
\hline & $\$ 000$ & 12,400 & 4,314 & 10,529 & 12,058 \\
\hline \multirow[t]{2}{*}{ Sugar } & Thousand Tons & 60.2 & 83.7 & 23.8 & 125.0 \\
\hline & $\$ 000$ & 11,265 & 26,450 & 7,500 & 29,323 \\
\hline \multirow[t]{2}{*}{ Tea } & Thousand Tons & 19.5 & 16.8 & 8.8 & 9.0 \\
\hline & $\$ 000$ & 46,896 & 34,626 & 18,636 & 24,968 \\
\hline \multirow[t]{2}{*}{ Vegetable Oil } & Thousand Tons & 19.5 & 38.8 & 50.2 & 46.5 \\
\hline & $\$ 000$ & 15,787 & 36,802 & 30,480 & 44,290 \\
\hline Beverages and Tobacco & $\$ 000$ & 4,103 & 15,191 & 11,435 & 11,802 \\
\hline Other Consumer Goods & $\$ 000$ & 96,049 & 252,555 & 167,945 & 164,206 \\
\hline Medicine \& Pharmaceuticals & $\$ 000$ & 9,341 & 9,128 & 6,453 & 8,552 \\
\hline \multirow[t]{2}{*}{ Textiles } & Million Meters & 53.4 & 130.9 & 102.6 & 76.9 \\
\hline & $\$ 000$ & 53,502 & 150,133 & 87,519 & 79,028 \\
\hline Threads & $\$ 000$ & 15,587 & 50,004 & 19,303 & 22,228 \\
\hline Others & $\$ 000$ & 17,619 & 43,290 & 54,670 & 54,398 \\
\hline Petroleum Products & $\$ 000$ & 50,692 & 154,712 & 133,687 & 92,351 \\
\hline Intermediate Goods & $\$ 000$ & 36,643 & 64,887 & 85,644 & 75,515 \\
\hline \multirow[t]{2}{*}{ Fertilizer } & Thousand Tons & 44.0 & 20.0 & 40.0 & 49.0 \\
\hline & $\$ 000$ & 6,333 & 4,240 & 9,842 & 12,800 \\
\hline Chemicals & $\$ 000$ & 7,151 & 5,843 & 4,383 & 5,366 \\
\hline Iron \& Steel & $\$ 000$ & & 15,594 & 25,613 & 18,617 \\
\hline Tires \& Tubes & $\$ 000$ & 23,159 & 39,210 & 45,806 & 38,722 \\
\hline Capital Goods & & 50,446 & 417,563 & 351,265 & 280,693 \\
\hline Commodities Not Classified & $\$ 000$ & 95,644 & 90,589 & 65,939 & 126,500 \\
\hline Total Imports & & 419,925 & $1,097,689$ & 883,060 & 861,706 \\
\hline
\end{tabular}

Source: GOA Central Statistical Office; cited from UNDP Discussion Paper on Afghanistan of April 1988. 
Table A V-20. Recorded Official Exports from Pakistan to

Afghanistan for Selected Products

(thousands of rupees)

\begin{tabular}{|c|c|c|c|c|c|c|c|c|c|c|c|}
\hline Product & 1978 & 1979 & 1980 & 1981 & 1982 & 1983 & 1984 & 1985 & 1986 & 1987 & 1988 \\
\hline Kino & 4,481 & 1,294 & 2,131 & 2,973 & 1,484 & 14,258 & 13,543 & 11,481 & 12,323 & 12,987 & 13,083 \\
\hline Bananas & 8,333 & 18,773 & 16,195 & 18,876 & 19,085 & 14,512 & 12,667 & 12,855 & 19,302 & 34,096 & 29,062 \\
\hline Mangoes & 137 & 99 & 122 & 224 & 251 & 172 & 567 & 329 & 59 & 601 & 3,489 \\
\hline Watermelons & 119 & 30 & 76 & 95 & 109 & 311 & 825 & 1,611 & 2,813 & 3,136 & 4,558 \\
\hline Sanuf supari & -- & -- & -- & -- & -- & -- & -- & -- & 0 & 2,505 & 20,210 \\
\hline Sweetmeats & 0 & 8 & -- & -- & -- & -- & -- & -- & 0 & 1,287 & 0 \\
\hline Sugar confections & -- & -- & 7 & 0 & 0 & 1,036 & 362 & 1,408 & 5,116 & 2,525 & 4,064 \\
\hline Chocolate preparations & -- & -- & -- & -- & -- & -- & 3,212 & 4,881 & 6,977 & 32,611 & 61,189 \\
\hline Biscuits (cookies) & -- & -- & -- & -- & -- & -- & - - & -- & 40 & 1,006 & 931 \\
\hline Lemonade & -- & -- & -- & -- & 493 & 0 & 2,956 & 1,355 & 4,480 & 5,754 & 3,165 \\
\hline Cigarettes & 16,897 & 28,192 & 29,447 & 33,121 & 61,608 & 107,505 & 109,142 & 144,430 & 171,960 & 195,708 & 295,525 \\
\hline Rock salt & 585 & 853 & 682 & 298 & 169 & 230 & 349 & 116 & 111 & 279 & 1,136 \\
\hline Cotton, unprocessed & -- & -- & -- & -- & -- & -- & 0 & 2,857 & 0 & 0 & 2,494 \\
\hline Finished leather & -- & -- & -- & -- & 2,055 & 840 & 1,757 & 1,595 & 3,165 & 16,202 & 19,833 \\
\hline Raw leather & -- & -- & -- & -- & 830 & 1,385 & 522 & 901 & 682 & 1,559 & 501 \\
\hline Wheat, rice, ghee, animals & -- & -- & -- & -- & -- & -- & -- & -- & -- & -- & -- \\
\hline Other agricultural products & 24,522 & 4,871 & 2,482 & 1,051 & 451 & 1,220 & 1,550 & 1,321 & 1,421 & 2,520 & 1,697 \\
\hline Total agricultural products & 55,074 & 54,120 & 51,142 & 56,638 & 86,535 & 141,469 & 147,452 & 185,140 & 228,449 & 312,776 & 460,937 \\
\hline Threshing machinery & -- & -- & 0 & 16 & 50 & 177 & 49 & 67 & 79 & 1,178 & 2,472 \\
\hline Tractors & -- & -- & -- & -- & -- & -- & -- & -- & -- & -- & -- \\
\hline N.S. agricultural machinery/parts & 254 & 55 & 13 & 68 & 16 & 97 & 362 & 491 & 121 & 1,218 & 721 \\
\hline Fertilizer & -- & -- & -- & -- & -- & -- & -- & -- & -- & -- & -- \\
\hline Seed & -- & -- & -- & -- & -- & -- & -- & -- & -- & -- & -- \\
\hline Total agricultural inputs & 254 & 55 & 13 & 84 & 66 & 274 & 411 & 558 & 200 & 2,396 & 3,193 \\
\hline Total fuels & -- & -- & -- & -- & -- & -- & -- & -- & -- & -- & -- \\
\hline Total selected exports & 55,328 & 54,175 & 51,155 & 56,722 & 86,601 & 141,743 & 147,863 & 185,698 & 228,649 & 315,172 & 464,130 \\
\hline
\end{tabular}

Source: Federal Bureau of Statistics, "Foreign Trade Statistics of Pakistan". 
Table A V-21. Principal Imports by Commodity

(millions of U.S. dollars)

\begin{tabular}{|c|c|c|c|c|c|c|c|c|c|c|}
\hline & $1966 / 67$ & $1967 / 68$ & $1968 / 69$ & $1969 / 70$ & $1970 / 71$ & $1971 / 72$ & $1972 / 73$ & $1973 / 74$ & $1974 / 75$ & $1975 / 76[a]$ \\
\hline Food \& live animals & 30.1 & 25.4 & 15.0 & 21.3 & 19.8 & 37.4 & 42.3 & 36.9 & 45.2 & 75.4 \\
\hline Wheat \& flour & 13.1 & 13.1 & 3.5 & 6.7 & 5.0 & 20.7 & 19.1 & 10.3 & 0.2 & 1.2 \\
\hline Sugar & 8.8 & 5.2 & 0.9 & 3.8 & 5.1 & 4.2 & 11.5 & 14.0 & 26.1 & 36.0 \\
\hline Tea & 6.1 & 4.9 & 9.5 & 9.4 & 7.9 & 9.9 & 9.6 & 10.1 & 15.7 & 29.8 \\
\hline Other & 2.1 & 2.2 & 1.1 & 1.4 & 1.8 & 2.6 & 2.1 & 2.5 & 3.2 & 8.4 \\
\hline Beverages \& tobacco & 0.4 & 0.4 & 0.5 & 0.5 & 0.5 & 0.9 & 0.7 & 1.0 & 1.6 & 1.9 \\
\hline Crude materials inedible except fuel & 0.0 & 0.2 & 0.1 & 0.0 & 0.0 & 0.2 & 0.3 & 2.5 & 7.6 & 5.7 \\
\hline $\begin{array}{l}\text { Mineral fuels, lubricants, \& } \\
\text { related materials }\end{array}$ & 5.6 & 5.4 & 6.4 & 7.5 & 6.8 & 8.4 & 9.5 & 11.1 & 22.5 & 25.5 \\
\hline Animal \& vegetable oils \& fats & 0.8 & 3.1 & 4.4 & 3.9 & 2.4 & 4.3 & 5.5 & 5.5 & 8.6 & 9.7 \\
\hline Chemicals & 4.3 & 4.8 & 5.0 & 7.6 & 7.6 & 12.0 & 14.2 & 27.6 & 24.0 & 7.7 \\
\hline Fertilizer & 0.0 & 0.0 & 0.0 & 2.0 & 1.5 & 4.0 & 5.6 & 16.8 & 8.8 & 0.1 \\
\hline Other & 4.3 & 4.8 & 5.0 & 5.6 & 6.1 & 8.0 & 8.6 & 10.8 & 15.2 & 7.6 \\
\hline Manufactured goods classified & & & & & & & & & & \\
\hline chiefly by material & 18.4 & 19.5 & 21.0 & 26.6 & 32.5 & 28.6 & 32.3 & 49.0 & 85.6 & 85.9 \\
\hline $\begin{array}{l}\text { Paper, paperboard, \& } \\
\text { manufactures thereof } \\
\text { Textile yarns, fabrics, made-up }\end{array}$ & 0.2 & 0.3 & 0.3 & 0.4 & 0.5 & 0.7 & 0.4 & 0.5 & 2.1 & 0.4 \\
\hline articles \& related products & 10.9 & 11.4 & 13.8 & 18.2 & 22.6 & 16.4 & 18.4 & 28.6 & 61.1 & 56.0 \\
\hline Other & 7.3 & 7.8 & 6.9 & 8.0 & 9.4 & 11.5 & 13.5 & 19.9 & 22.4 & 29.5 \\
\hline Machinery \& trans. equip. & 8.4 & 8.2 & 8.1 & 10.0 & 12.4 & 15.7 & 13.1 & 16.2 & 19.2 & 23.5 \\
\hline Machinery & 3.9 & 3.4 & 4.0 & 4.2 & 4.6 & 6.0 & 4.3 & 6.6 & 8.7 & \\
\hline Road motor vehicles & 4.1 & 4.4 & 3.8 & 5.4 & 7.1 & 9.2 & 8.6 & 9.2 & 9.8 & \\
\hline Other trans. equip. & 0.4 & 0.4 & 0.3 & 0.4 & 0.7 & 0.5 & 0.2 & 0.4 & 0.7 & \\
\hline Misc. manufactured articles & 6.7 & 7.0 & 8.6 & 8.1 & 9.4 & 7.3 & 7.9 & 10.3 & 9.0 & 4.8 \\
\hline $\begin{array}{l}\text { Commodities \& transactions not } \\
\text { classified by kind }\end{array}$ & 76.1 & 64.4 & 55.3 & 39.3 & 19.4 & 38.4 & 31.2 & 22.8 & 19.2 & 37.4 \\
\hline Total Recorded Imports & 150.8 & 138.4 & 124.4 & 124.8 & 110.8 & 153.2 & 157.0 & 182.9 & 242.5 & 277.5 \\
\hline
\end{tabular}

Note: CIF values.

[a] Preliminary

Sources: GOA Da Afghanistan Bank and Central Statistical Office; cited from "Afghanistan: The Journey to Economic Development",

Vol. II, March 1978, World Bank. 
Table A V-22. Imports by Region

(millions of U.S. dollars)

\begin{tabular}{|c|c|c|c|c|c|c|c|c|c|}
\hline & $1966 / 67$ & $1967 / 68$ & $1968 / 69$ & $1969 / 70$ & $1970 / 71$ & $1971 / 72$ & $1972 / 73$ & $1973 / 74$ & $1974 / 75$ \\
\hline \multicolumn{10}{|l|}{ Multilateral Countries } \\
\hline \multicolumn{10}{|l|}{ OECD Countries } \\
\hline USA & 26.0 & 17.7 & 10.5 & 7.0 & 3.6 & 18.0 & 16.5 & 22.0 & 10.5 \\
\hline UK & 2.2 & 3.6 & 3.2 & 5.2 & 5.4 & 10.6 & 5.7 & 7.7 & 8.4 \\
\hline Germany & 7.3 & 11.2 & 11.3 & 7.0 & 7.5 & 10.9 & 14.2 & 11.2 & 10.7 \\
\hline Japan & 9.6 & 10.6 & 12.0 & 14.6 & 19.3 & 19.6 & 24.2 & 31.7 & 59.7 \\
\hline Other & 3.6 & 5.2 & 5.8 & 8.3 & 7.8 & 8.5 & 10.0 & 11.0 & 13.7 \\
\hline Total OECD & 48.7 & 48.3 & 42.8 & 42.1 & 43.6 & 67.6 & 70.6 & 83.6 & 103.0 \\
\hline \multicolumn{10}{|l|}{ Other multilateral } \\
\hline India & 6.8 & 6.3 & 11.4 & 11.4 & 12.9 & 11.4 & 11.7 & 18.9 & 24.6 \\
\hline Pakistan & 4.6 & 3.3 & 3.1 & 3.6 & 3.3 & 3.6 & 6.4 & 5.5 & 10.8 \\
\hline Iran & 0.6 & 2.9 & 4.9 & 3.6 & 2.8 & 5.3 & 10.2 & 4.9 & 13.1 \\
\hline Other & 2.8 & 4.3 & 4.1 & 11.6 & 3.2 & 17.2 & 14.6 & 23.6 & 13.9 \\
\hline Total other multilateral & 14.8 & 16.8 & 23.5 & 30.2 & 22.2 & 37.5 & 42.9 & 52.9 & 62.4 \\
\hline Total multilateral & 63.5 & 65.1 & 66.3 & 72.3 & 65.8 & 105.1 & 113.5 & 136.5 & 165.4 \\
\hline \multicolumn{10}{|l|}{ Bilateral countries } \\
\hline USSR & 80.8 & 66.5 & 47.8 & 41.7 & 37.9 & 40.1 & 38.5 & 38.3 & 73.3 \\
\hline China & 3.1 & 5.6 & 9.3 & 10.3 & 6.2 & 7.4 & 4.6 & 7.4 & 3.0 \\
\hline Other & 3.4 & 1.2 & 1.0 & 0.5 & 0.9 & 0.6 & 0.4 & 0.7 & 0.8 \\
\hline Total bilateral & 87.3 & 73.3 & 58.1 & 52.5 & 45.0 & 48.1 & 43.5 & 46.4 & 77.1 \\
\hline Total recorded imports & 150.8 & 138.4 & 124.4 & 124.8 & 110.8 & 153.2 & 157.0 & 182.9 & 242.5 \\
\hline
\end{tabular}

Note: CIF values.

Sources: GOA Da Afghanistan Bank and Central Statistical Office; cited from "Afghanistan: The Journey to Economic Development",

Vol. II, March 1978, World Bank. 
Table A V-23. Free Market and Related Exchange Rates (Average rates in Afghanis per U.S. Dollar)

\begin{tabular}{|c|c|c|c|c|c|c|c|}
\hline \multirow[b]{2}{*}{ Yr. ended March 20} & \multicolumn{3}{|c|}{ Bazaar } & \multicolumn{2}{|c|}{ Da Afghanistan Bank } & \multirow{2}{*}{\multicolumn{2}{|c|}{ Selling }} \\
\hline & Buying & Middle & Selling & Buying Middle & & & \\
\hline \multicolumn{8}{|l|}{ Annual Averages } \\
\hline $1972 / 73$ & -- & 80.50 & -- & -- & 80.60 & & -- \\
\hline $1973 / 74$ & -- & 60.72 & -- & -- & 61.05 & . & -- \\
\hline $1974 / 75$ & -- & 56.58 & -- & -- & 58.21 & & -- \\
\hline $1975 / 76$ & -- & 55.04 & -- & -- & 57.66 & & -- \\
\hline $1976 / 77$ & -- & 47.38 & -- & -- & 54.10 & & -- \\
\hline $1977 / 78$ & -- & -- & -- & -- & -- & & -- \\
\hline $1978 / 79$ & -- & 38.80 & -- & -- & -- & & -- \\
\hline $1979 / 80$ & -- & 42.90 & -- & -- & -- & & -- \\
\hline $1980 / 81$ & -- & 46.10 & -- & -- & -- & & -- \\
\hline $1981 / 82$ & -- & 56.60 & -- & -- & 50.20 & & -- \\
\hline $1982 / 83$ & -- & 76.80 & -- & -- & 50.60 & & -- \\
\hline $1983 / 84$ & -- & 99.30 & -- & -- & 50.60 & & -- \\
\hline $1984 / 85$ & -- & 122.00 & -- & -- & 50.60 & & -- \\
\hline $1985 / 86$ & -- & 136.30 & -- & -- & 50.60 & & -- \\
\hline $1986 / 87[b]$ & -- & 144.70 & -- & -- & 50.60 & & -- \\
\hline \multicolumn{8}{|c|}{ 1975/76 Monthly Average [c] } \\
\hline March/April & 54.69 & 54.94 & 55.19 & 55.62 & 56.62 & & 57.62 \\
\hline April/May & 55.05 & 55.30 & 55.55 & 56.50 & 57.50 & & 58.50 \\
\hline May/June & 54.91 & 55.16 & 55.41 & 56.50 & 57.50 & & 58.50 \\
\hline June/July & 55.12 & 55.37 & 55.62 & 56.50 & 57.50 & & 58.50 \\
\hline July/August & 55.62 & 55.87 & 56.12 & 56.96 & 57.96 & & 58.96 \\
\hline August/September & 54.35 & 54.60 & 54.85 & 57.00 & 58.00 & & 59.00 \\
\hline September/October & 54.34 & 54.59 & 54.84 & 57.00 & 58.00 & & 59.00 \\
\hline October/November & 54.17 & 54.42 & 54.67 & 57.00 & 58.00 & & 59.00 \\
\hline November/December & 54.28 & 54.53 & 54.78 & 57.00 & 58.00 & & 59.00 \\
\hline December/January & 54.70 & 54.94 & 55.18 & 57.00 & 57.84 & & 58.68 \\
\hline January/February & 55.47 & 55.44 & 56.01 & 57.00 & 57.50 & & 58.00 \\
\hline February/March & 54.73 & 55.00 & 55.28 & 57.00 & 57.50 & & 58.00 \\
\hline \multicolumn{8}{|c|}{ 1976/77 Monthly Average [c] } \\
\hline March/April & 53.99 & 54.24 & 54.49 & 56.50 & 57.00 & & 57.50 \\
\hline April/May & 53.03 & 53.16 & 53.53 & 56.32 & 56.82 & & 57.32 \\
\hline May/June & 50.51 & 50.76 & 51.01 & 56.00 & 56.50 & & 57.00 \\
\hline June/July & 47.49 & 47.73 & 47.07 & 56.00 & 56.50 & & 57.00 \\
\hline July/August & 46.35 & 46.55 & 46.76 & 56.00 & 56.50 & & 57.00 \\
\hline August/September & 44.20 & 44.42 & 44.65 & 56.00 & 56.50 & & 57.00 \\
\hline September/October & 41.45 & 41.66 & 41.87 & 56.00 & 56.50 & & 57.00 \\
\hline October/November & 45.84 & 46.07 & 46.30 & 50.48 & 50.98 & & 51.48 \\
\hline November/December & 43.88 & 44.12 & 44.36 & 50.00 & 50.50 & & 51.00 \\
\hline December/January & 45.73 & 45.98 & 46.24 & 50.00 & 50.50 & & 51.00 \\
\hline January/February & 46.69 & 46.95 & 47.22 & 50.00 & 50.50 & & 51.00 \\
\hline February/March & 46.65 & 46.79 & 46.93 & 50.00 & 50.50 & & 51.00 \\
\hline \multicolumn{8}{|c|}{ 1988/89 Monthly Average [d] } \\
\hline July/August & 187.66 & 187.05 & 186.45 & -- & -- & & -- \\
\hline August/September & 192.90 & 192.15 & 191.40 & -- & -- & & -- \\
\hline September/October & 196.82 & 196.04 & 195.26 & -- & -- & & -- \\
\hline October/November & 191.68 & 190.88 & 190.08 & -- & -- & & -- \\
\hline November/December & 198.14 & 197.35 & 196.57 & -- & -- & & -- \\
\hline December/January & 197.80 & 197.16 & 196.53 & -- & -- & & -- \\
\hline January/February & 205.05 & 204.30 & 203.56 & -- & -- & & -- \\
\hline February/March & 203.05 & 202.16 & 201.27 & -- & -- & & -- \\
\hline March/April & 221.58 & 220.44 & 219.32 & -- & -- & & -- \\
\hline April/May & 248.35 & 246.71 & 245.09 & -- & -- & & -- \\
\hline May/June & 293.71 & 291.16 & 288.65 & -- & -- & & -- \\
\hline June/July & 322.40 & 320.45 & 318.52 & -- & -- & & -- \\
\hline
\end{tabular}

[a] Until January 1976 commercial banks maintained exchange rates identical to Da Afghanistan Bank.

[b] Average for first half of the year.

[c] Month ending on the 20th or 21 st day.

[d] Monthly average of weekly rates.

Sources: Da Afghanistan Bank; cited from "Afghanistan: The Journey to Economic Development", Vol. II, March 1978, World Bank; IMF "Recent Economic Developments", December 1986; Zarab Sarafa Association.

\begin{tabular}{|c|c|c|c|c|}
\hline & 9.67 & 9.70 & 9.73 & 18.15 \\
\hline & 9.51 & 9.55 & 9.59 & 18.35 \\
\hline & 9.43 & 9.46 & 9.50 & 18.55 \\
\hline & 9.39 & 9.43 & 9.47 & 18.00 \\
\hline & 9.41 & 9.45 & 9.49 & 18.65 \\
\hline & 9.66 & 9.69 & 9.72 & 19.10 \\
\hline & 9.41 & 9.45 & 9.48 & 19.30 \\
\hline & 9.73 & 9.77 & 9.81 & 19.75 \\
\hline & 9.09 & 9.14 & 9.19 & 20.15 \\
\hline & 8.23 & 8.29 & 8.34 & 20.45 \\
\hline & 7.13 & 7.20 & 7.26 & 20.95 \\
\hline & 6.67 & 6.71 & 6.75 & 21.50 \\
\hline
\end{tabular}




\section{LABOR FORCE AND POPULATION}

1. Population Est., by Source

2. Comparative Pop. Est.

3. RRNA Pop. Projections, 1979-88

4. Pop. and Rate of Growth (UN)

5. Total Pop., 1979 (U.S. Census)

6. Official Census Pop. Est.

7. Pop. and Rates of Growth (IBRD)

8. Origin of Refugees (UNHCR)

9. GOA Est. of Total Pop. (East)

10. GOA Est. of Rural Pop. (East)

11. GOA Est. of Urban Pop. (East)
12. GOA Est. of Total Pop. (North)

13. Char. of Work Force (IBRD)

14. Labor Force Composition (IBRD)

15. Labor Force, 1969

16. Pre-war Labor Force

17. Changes in Farm Size (North)

18. Socio-political Stratification

19. Civil Administration Div.

20. Enrollment Growth in Educ.

21. Selected Social Indicators

O. RETURN TO MAIN MENU 


\begin{tabular}{|c|c|c|c|c|c|c|c|c|c|c|c|c|c|c|c|c|c|c|c|}
\hline & 1970 & 1971 & 1972 & 1973 & 1974 & 1975 & 1976 & 1977 & 1978 & 1979 & 1980 & 1981 & 1982 & 1983 & 1984 & 1985 & 1986 & 1987 & 1988 \\
\hline World Bank Series [a] & -- & -- & 12,840 & 13,120 & 13,410 & 13,700 & 14,000 & -- & -- & -- & -- & -- & -- & -- & -- & -- & -- & -- & -- \\
\hline U.S. Census Bureau [b] & 12,431 & -- & -- & -- & -- & 14,132 & 14,501 & 14,880 & 15,269 & 15,556 & 14,985 & 14,085 & 13,642 & 13,703 & 13,817 & 13,886 & 13,981 & 14,184 & 14,481 \\
\hline $\begin{array}{l}\text { RRNA Total Population } \\
\text { Estimates [c] }\end{array}$ & -- & -- & -- & -- & -- & -- & -- & -- & -- & -- & 12,925 & 11,942 & 10,941 & 10,683 & 10,419 & 10,130 & 9,835 & 9,683 & 9,528 \\
\hline UNHCR [d] & -- & -- & -- & -- & -- & -- & -- & -- & 14,210 & -- & -- & -- & -- & -- & -- & -- & -- & 10,980 & -- \\
\hline GOA Official Estimates [e] & -- & -- & -- & -- & -- & -- & -- & -- & -- & 13,051 & -- & -- & -- & -- & 17,672 & 18,136 & 18,614 & -- & -- \\
\hline
\end{tabular}

[a] Total population, including nomads.

b] Settled population only, excluding nomads.

c) Based on official GOA census of 1979 of total population. See Table A VI-3 for derivation of these figures.

d] UN estimate of total population inside Afghanistan

e) Figure for 1979 excludes nomads. For 1984-86, figures include official GOA estimates of nomadic population, but do not account for emigration by refugees. These figures

are cited from The Europa Yearbook 1988, Vol. I, Europa Publications Ltd. 


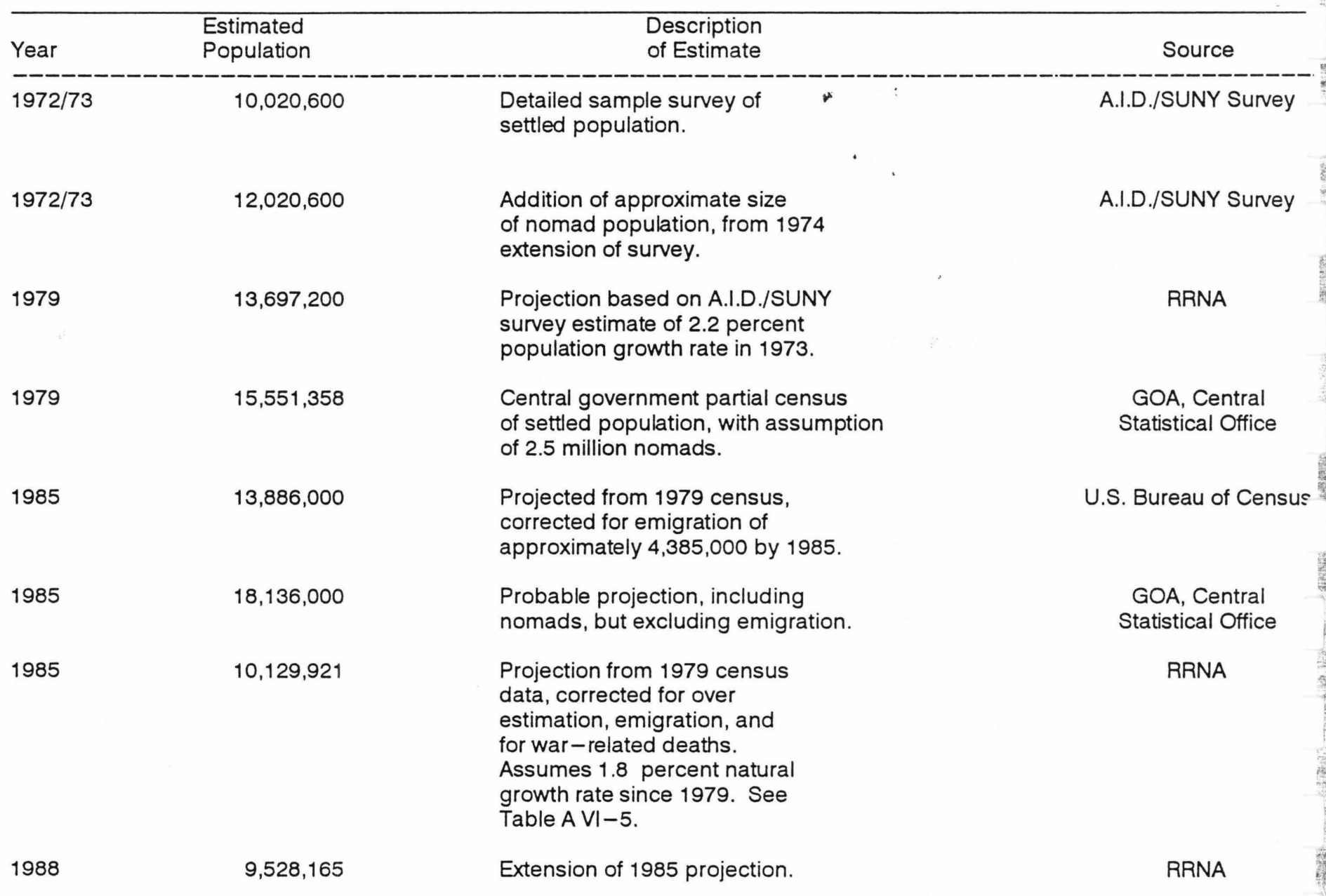


Table A VI-3. RRNA Population Projections, 1979-88

\begin{tabular}{|c|c|c|c|c|c|c|c|c|c|c|}
\hline & 1979 & 1980 & 1981 & 1982 & 1983 & 1984 & 1985 & 1986 & 1987 & 1988 \\
\hline $\begin{array}{l}\text { 1. Official GOA Census } \\
\text { (settled population) }\end{array}$ & $13,051,358$ & & & & & & & & & \\
\hline 2. Plus Official GOA Estimate, Nomads & $2,500,000$ & & & & & & & & & \\
\hline 3. Total GOA Estimate & $15,551,358$ & & & & & & & & & \\
\hline $\begin{array}{l}\text { 4. Less Correction Factor for } \\
\text { Over-enumeration [a] }\end{array}$ & $-10.0 \%$ & & & & & & & & & \\
\hline 5. Corrected 1979 Total Population & $13,996,222$ & & & & & & & & & \\
\hline 6. Less War-related Deaths [b] & & 111,111 & 111,111 & 111,111 & 111,111 & 111,111 & 111,111 & 111,111 & 111,111 & 111,111 \\
\hline 7. Less Emigration [c] & & $1,189,000$ & $1,083,000$ & $1,083,000$ & 336,000 & 337,000 & 357,000 & 358,000 & 212,000 & 212,000 \\
\hline 8. Population Base Prior to Natural Increase [d] & & $12,696,111$ & $11,730,530$ & $10,747,568$ & $10,498,914$ & $10,234,693$ & $9,950,806$ & $9,660,810$ & $9,511,593$ & $9,359,691$ \\
\hline 9. Plus, Natural Increase & & 228,530 & 211,150 & 193,456 & 188,890 & 184,224 & 179,115 & 173,895 & 171,209 & 168,474 \\
\hline 10. Revised Estimated Total Population & $13,996,222$ & $12,924,641$ & $11,941,680$ & $10,941,025$ & $10,682,804$ & $10,418,917$ & $10,129,921$ & $9,834,704$ & $9,682,802$ & $9,528,165$ \\
\hline
\end{tabular}

[a] Modal interview response.

[b] Assumes total war-related deaths through 1988 of 1 million.

[c] Based on the U.S. Bureau of Census projection of the number of refugees living in Iran and Pakistan as of $1988--5,167,000$; consistent with UNHCR count.

[d] Figure in item 10 for preceding year less items 6 and 7 .

[e] Low-end growth rate accepted by the GOA. The high-end estimate is 2.2 percent.

Sources: GOA Central Statistical Office; "Afghanistan: A Demographic Profile", Frank Hobbs, U.S. Bureau of the Census, January 1988; RRNA. 
Table A VI-4. UNDP Series on Population and Rate of Growth

(1978/79 and 1987/88)

(thousands)

\begin{tabular}{|c|c|c|}
\hline & $\begin{array}{l}\text { Official } \\
\text { Estimate }\end{array}$ & $\begin{array}{l}\text { Revised } \\
\text { Estimate }\end{array}$ \\
\hline Settled Population (1978/79) & 12,710 & 12,710 \\
\hline Rural & 10,830 & 10,830 \\
\hline Urban & 1,880 & 1,880 \\
\hline Nomads $(1978 / 79)$ & 1,500 & 1,500 \\
\hline Total Population (1978/79) & 14,210 & 14,210 \\
\hline National Growth Rate & $1.8 \%$ & $2.0 \%$ \\
\hline Total Population (1987/88) & 16,726 & 16,980 \\
\hline Less War Related Deaths & -- & 1,000 \\
\hline Less Refugees in Pakistan & -- & 3,500 \\
\hline$-N W F P$ & -- & 2,158 \\
\hline -Punjab & -- & 180 \\
\hline -Baluchistan & -- & 818 \\
\hline -Unregistered & -- & 344 \\
\hline Less Refugees in Iran & -- & 1,400 \\
\hline Less Refugees in Europe, USA, India, etc. & -- & 100 \\
\hline Total Adjustment & -- & 6,000 \\
\hline $\begin{array}{l}\text { Total Population inside Afghanistan: } \\
\text { Of Which: }\end{array}$ & 16,726 & 10,980 \\
\hline Settled & $15,226[a]$ & $9,992[b]$ \\
\hline Rural & 12,574 & 7,212 \\
\hline Urban & 2,652 & 2,780 \\
\hline Kabul & 1,348 & 2,000 \\
\hline \multirow[t]{2}{*}{ Others } & 1,304 & 780 \\
\hline & 1,500 & 988 \\
\hline
\end{tabular}

Note: These figures differ substantially from those of other secondary publications citing "official" primary sources. See text.

[a] Official Data

[b] Estimated, using the same ratios as in official estimates.

Sources: Central Statistical Office, UNHCR, Government of Pakistan, and Afghanistan organizations in Pakistan and Iran; cited from UNDP Discussion Paper on Afghanistan of April 1988. 
Table A VI-5. U.S. Census Bureau Series on Total and Urban Population by Province, 1979

\begin{tabular}{|c|c|c|c|}
\hline Province & Total & Urban & $\begin{array}{r}\text { Urban as \% } \\
\text {. of Total }\end{array}$ \\
\hline Badakshan & 497,758 & 10,142 & $\cdot 2.04 \%$ \\
\hline Badghes & 233,613 & 5,340 & $2.29 \%$ \\
\hline Baghlan & 493,882 & 75,130 & $15.21 \%$ \\
\hline Balkh & 569,255 & 122,567 & $21.53 \%$ \\
\hline Bamyan & 268,517 & 7,355 & $2.74 \%$ \\
\hline Farah & 234,621 & 18,797 & $8.01 \%$ \\
\hline Faryab & 582,705 & 54,954 & $9.43 \%$ \\
\hline Ghazni & 646,623 & 30,425 & $4.71 \%$ \\
\hline Ghor & 337,992 & 2,974 & $0.88 \%$ \\
\hline Helmand & 517,645 & 26,646 & $5.15 \%$ \\
\hline Herat & 769,111 & 163,960 & $21.32 \%$ \\
\hline Jowzjan & 588,609 & 54,870 & $9.32 \%$ \\
\hline Kabul & $1,864,000$ & 919,108 & $49.31 \%$ \\
\hline Kandahar & 567,204 & 178,409 & $31.45 \%$ \\
\hline Kunarha & 250,132 & 2,089 & $0.84 \%$ \\
\hline Kunduz & 555,437 & 107,191 & $19.30 \%$ \\
\hline Laghman & 310,751 & 3,987 & $1.28 \%$ \\
\hline Nangarhar & 745,986 & 56,384 & $7.56 \%$ \\
\hline Nimroz & 103,634 & 6,477 & $6.25 \%$ \\
\hline Parwan & 755,285 & 25,093 & $3.32 \%$ \\
\hline Paktika & 245,229 & 1,398 & $0.57 \%$ \\
\hline Paktia & 497,503 & 11,415 & $2.29 \%$ \\
\hline Samangan & 272,584 & 33,016 & $12.11 \%$ \\
\hline Takhar & 519,752 & 46,202 & $8.89 \%$ \\
\hline Uruzgan & 444,168 & 6,863 & $1.55 \%$ \\
\hline Zabul & 179,362 & 5,946 & $3.32 \%$ \\
\hline Total & $13,051,358$ & $1,976,738$ & $15.15 \%$ \\
\hline
\end{tabular}

Census Bureau Note: Data refer to the settled population only. "Urban" initially referred to the 86 areas recognized by the Central Bureau for Leading Local Organs as urban and deserving of municipal status, but during processing of the census results 23 of these areas were determined to be "too small and lacking the required urban features" to warrant classification as urban. Hence, "urban" was operationally defined as 63 areas recognized as such by the Central Bureau for Leading Local Organs. These areas met unspecified requirements as to size and urban features.

Sources: Afghanistan Central Statistics Office, 1981, Preliminary results of the First Afghan

Population Census, Publication No. 1, Demographic

Statistics, Kabul, annex tables 1, 1-1, and 1-2; cited from "Afghanistan: A Demographic Profile", January 1988, U.S. Bureau of the Census. 
Table A VI-6. Official Census Population Estimates for

Afghanistan by Province and District, 1978/79

\begin{tabular}{|c|c|c|c|c|c|c|}
\hline Province & Woliswali & Urban & $\%$ & Rural & $\%$ & Total \\
\hline Badakhshan & $\begin{array}{l}\text { Baharak } \\
\text { Darwaz } \\
\text { Eshkashem } \\
\text { Faizabad } \\
\text { Jurm } \\
\text { Keranomanjan } \\
\text { Keshem } \\
\text { Ragh } \\
\text { Shahre Bozurg } \\
\text { Shighnan } \\
\text { Wakhan } \\
\text { Zebak }\end{array}$ & $\begin{array}{r}0 \\
0 \\
0 \\
9,121 \\
1,054 \\
0 \\
0 \\
0 \\
0 \\
0 \\
0 \\
0\end{array}$ & $\begin{array}{l}0.0 \% \\
0.0 \% \\
0.0 \% \\
6.5 \% \\
2.1 \% \\
0.0 \% \\
0.0 \% \\
0.0 \% \\
0.0 \% \\
0.0 \% \\
0.0 \% \\
0.0 \%\end{array}$ & $\begin{array}{r}46,093 \\
51,829 \\
7,286 \\
131,207 \\
49,136 \\
5,590 \\
83,204 \\
62,227 \\
28,994 \\
18,241 \\
9,178 \\
4,608\end{array}$ & $\begin{array}{r}100.0 \% \\
100.0 \% \\
100.0 \% \\
93.5 \% \\
97.9 \% \\
100.0 \% \\
100.0 \% \\
100.0 \% \\
100.0 \% \\
100.0 \% \\
100.0 \% \\
100.0 \%\end{array}$ & $\begin{array}{r}46,093 \\
51,829 \\
7,286 \\
140,328 \\
50,190 \\
5,590 \\
83,204 \\
62,227 \\
28,994 \\
18,241 \\
9,178 \\
4,608\end{array}$ \\
\hline Total Badakhshan & & 10,175 & $2.0 \%$ & 497,593 & $98.0 \%$ & 507,768 \\
\hline Badghis & $\begin{array}{l}\text { Ghormach } \\
\text { Jawand } \\
\text { Khoshke Kohna } \\
\text { Morghab } \\
\text { Qades } \\
\text { Qalaenaw }\end{array}$ & $\begin{array}{r}0 \\
0 \\
0 \\
0 \\
0 \\
5,338\end{array}$ & $\begin{array}{l}0.0 \% \\
0.0 \% \\
0.0 \% \\
0.0 \% \\
0.0 \% \\
8.0 \%\end{array}$ & $\begin{array}{l}27,129 \\
36,938 \\
24,087 \\
35,003 \\
43,725 \\
61,393\end{array}$ & $\begin{array}{r}100.0 \% \\
100.0 \% \\
100.0 \% \\
100.0 \% \\
100.0 \% \\
92.0 \%\end{array}$ & $\begin{array}{l}27,129 \\
36,938 \\
24,087 \\
35,003 \\
43,725 \\
66,731\end{array}$ \\
\hline Total Badghis & & 5,338 & $2.3 \%$ & 228,275 & $97.7 \%$ & 233,613 \\
\hline Baghlan & $\begin{array}{l}\text { Andrab } \\
\text { Borka } \\
\text { Dahane Ghori } \\
\text { Doshi } \\
\text { Khenjan } \\
\text { Khost Wafreng } \\
\text { Markizbaghlan } \\
\text { Nahrin } \\
\text { Puli Khumri } \\
\text { Tala Wabarfak }\end{array}$ & $\begin{array}{r}0 \\
0 \\
1,558 \\
0 \\
0 \\
0 \\
39,212 \\
3,230 \\
31,090 \\
0\end{array}$ & $\begin{array}{r}0.0 \% \\
0.0 \% \\
4.1 \% \\
0.0 \% \\
0.0 \% \\
0.0 \% \\
35.4 \% \\
6.6 \% \\
31.7 \% \\
0.0 \%\end{array}$ & $\begin{array}{l}49,281 \\
34,259 \\
36,453 \\
37,600 \\
16,849 \\
46,746 \\
71,555 \\
45,704 \\
66,986 \\
14,359\end{array}$ & $\begin{array}{r}100.0 \% \\
100.0 \% \\
95.9 \% \\
100.0 \% \\
100.0 \% \\
100.0 \% \\
64.6 \% \\
93.4 \% \\
68.3 \% \\
100.0 \%\end{array}$ & $\begin{array}{r}49,281 \\
34,259 \\
38,011 \\
37,600 \\
16,849 \\
46,746 \\
110,767 \\
48,934 \\
98,076 \\
14,359\end{array}$ \\
\hline Total Baghlan & & 75,090 & $15.2 \%$ & 419,792 & $84.8 \%$ & 494,882 \\
\hline Balkh & $\begin{array}{l}\text { Balkh } \\
\text { Char Cant } \\
\text { Charbolak } \\
\text { Chemtal } \\
\text { Dihdadi } \\
\text { Dowlat Abad } \\
\text { Keshende } \\
\text { Nahre Shahi } \\
\text { Sholgera } \\
\text { Shortepa }\end{array}$ & $\begin{array}{r}7,275 \\
0 \\
0 \\
0 \\
0 \\
6,082 \\
0 \\
0 \\
5,862 \\
0\end{array}$ & $\begin{array}{r}10.4 \% \\
0.0 \% \\
0.0 \% \\
0.0 \% \\
0.0 \% \\
9.3 \% \\
0.0 \% \\
0.0 \% \\
8.0 \% \\
0.0 \%\end{array}$ & $\begin{array}{l}62,674 \\
29,506 \\
45,522 \\
49,963 \\
32,529 \\
59,315 \\
42,737 \\
32,017 \\
67,418 \\
24,983\end{array}$ & $\begin{array}{r}89.6 \% \\
100.0 \% \\
100.0 \% \\
100.0 \% \\
100.0 \% \\
90.7 \% \\
100.0 \% \\
100.0 \% \\
92.0 \% \\
100.0 \%\end{array}$ & $\begin{array}{l}69,949 \\
29,506 \\
45,522 \\
49,963 \\
32,529 \\
65,397 \\
42,737 \\
32,017 \\
73,280 \\
24,983\end{array}$ \\
\hline Total Balkh & & 19,219 & $4.1 \%$ & 446,664 & $95.9 \%$ & 465,883 \\
\hline Bamyan & $\begin{array}{l}\text { Kahmard } \\
\text { Markiz Bamyan } \\
\text { Panjab } \\
\text { Shiber } \\
\text { Waras } \\
\text { Yakowlang }\end{array}$ & $\begin{array}{r}0 \\
7,340 \\
0 \\
18 \\
0 \\
0\end{array}$ & $\begin{array}{r}0.0 \% \\
15.7 \% \\
0.0 \% \\
0.1 \% \\
0.0 \% \\
0.0 \%\end{array}$ & $\begin{array}{l}32,314 \\
39,413 \\
45,747 \\
18,422 \\
69,675 \\
55,588\end{array}$ & $\begin{array}{r}100.0 \% \\
84.3 \% \\
100.0 \% \\
99.9 \% \\
100.0 \% \\
100.0 \%\end{array}$ & $\begin{array}{l}32,314 \\
46,753 \\
45,747 \\
18,440 \\
69,675 \\
55,588\end{array}$ \\
\hline Total Bamyan & & 7,359 & $2.7 \%$ & 261,158 & $97.3 \%$ & 268,517 \\
\hline Farah & $\begin{array}{l}\text { Anar Dara } \\
\text { Bakwa } \\
\text { Bala Blouk } \\
\text { Farsi } \\
\text { Gulistan } \\
\text { Khake Safid } \\
\text { Markiz Farah } \\
\text { Purchaman } \\
\text { Qalae Kah }\end{array}$ & $\begin{array}{r}0 \\
0 \\
0 \\
0 \\
0 \\
0 \\
18,765 \\
0 \\
0\end{array}$ & $\begin{array}{r}0.0 \% \\
0.0 \% \\
0.0 \% \\
0.0 \% \\
0.0 \% \\
0.0 \% \\
25.7 \% \\
0.0 \% \\
0.0 \%\end{array}$ & $\begin{array}{l}15,331 \\
13,844 \\
33,223 \\
24,066 \\
16,101 \\
11,641 \\
54,251 \\
26,682 \\
26,381\end{array}$ & $\begin{array}{r}100.0 \% \\
100.0 \% \\
100.0 \% \\
100.0 \% \\
100.0 \% \\
100.0 \% \\
74.3 \% \\
100.0 \% \\
100.0 \%\end{array}$ & $\begin{array}{l}15,331 \\
13,844 \\
33,223 \\
24,066 \\
16,101 \\
11,641 \\
73,016 \\
26,682 \\
26,381\end{array}$ \\
\hline
\end{tabular}


Table A VI-6 (cont.). Official Census Population Estimates for Afghanistan by Province and District, 1978/79

\begin{tabular}{|c|c|c|c|c|c|c|}
\hline Province & Woliswali & Urban & $\%$ & Rural & $\%$ & Total \\
\hline Farah (cont.) & Shindand & 2,091 & $1.8 \%$ & 114,101 & $98.2 \%$ & 116,192 \\
\hline Total Farah & & 20,857 & $5.9 \%$ & 335,620 & $94.1 \%$ & 356,477 \\
\hline Faryab & $\begin{array}{l}\text { Almar } \\
\text { Andkhoy } \\
\text { Bilchragh } \\
\text { Darzab } \\
\text { Dowlatabad } \\
\text { Khanchar Bagh } \\
\text { Kohistanat } \\
\text { Mimana } \\
\text { Pashton Kot } \\
\text { Qaram Qul } \\
\text { Qaysar } \\
\text { Shirin Tagab }\end{array}$ & $\begin{array}{r}0 \\
13,158 \\
0 \\
1,148 \\
2,440 \\
0 \\
0 \\
38,251 \\
0 \\
0 \\
0 \\
0\end{array}$ & $\begin{array}{r}0.0 \% \\
26.4 \% \\
0.0 \% \\
2.8 \% \\
9.1 \% \\
0.0 \% \\
0.0 \% \\
100.0 \% \\
0.0 \% \\
0.0 \% \\
0.0 \% \\
0.0 \%\end{array}$ & $\begin{array}{r}38,350 \\
36,684 \\
69,243 \\
39,851 \\
24,372 \\
15,244 \\
26,212 \\
0 \\
114,733 \\
11,719 \\
84,091 \\
67,209\end{array}$ & $\begin{array}{r}100.0 \% \\
73.6 \% \\
100.0 \% \\
97.2 \% \\
90.9 \% \\
100.0 \% \\
100.0 \% \\
0.0 \% \\
100.0 \% \\
100.0 \% \\
100.0 \% \\
100.0 \%\end{array}$ & $\begin{array}{r}38,350 \\
49,842 \\
69,243 \\
40,999 \\
26,812 \\
15,244 \\
26,212 \\
38,251 \\
114,733 \\
11,719 \\
84,091 \\
67,209\end{array}$ \\
\hline Total Faryab & & 54,997 & $9.4 \%$ & 527,708 & $90.6 \%$ & 582,705 \\
\hline Ghazni & $\begin{array}{l}\text { Ab Band } \\
\text { Ajristan } \\
\text { Andar } \\
\text { Bahram } \\
\text { Dehyak } \\
\text { Gelan } \\
\text { Giro } \\
\text { Jaghatu } \\
\text { Jaghori } \\
\text { Malistan } \\
\text { Markaz Ghazni } \\
\text { Moqor } \\
\text { Nawa } \\
\text { Nawor } \\
\text { Qarabagh } \\
\text { Zena Khan }\end{array}$ & $\begin{array}{r}0 \\
0 \\
0 \\
0 \\
0 \\
0 \\
0 \\
0 \\
0 \\
0 \\
30,459 \\
0 \\
0 \\
0 \\
0 \\
0\end{array}$ & $\begin{array}{r}0.0 \% \\
0.0 \% \\
0.0 \% \\
0.0 \% \\
0.0 \% \\
0.0 \% \\
0.0 \% \\
0.0 \% \\
0.0 \% \\
0.0 \% \\
41.0 \% \\
0.0 \% \\
0.0 \% \\
0.0 \% \\
0.0 \% \\
0.0 \%\end{array}$ & $\begin{array}{r}13,723 \\
16,137 \\
62,109 \\
31,704 \\
25,764 \\
30,796 \\
15,593 \\
51,703 \\
99,126 \\
45,377 \\
43,832 \\
25,357 \\
17,150 \\
54,173 \\
76,401 \\
7,219\end{array}$ & $\begin{array}{r}100.0 \% \\
100.0 \% \\
100.0 \% \\
100.0 \% \\
100.0 \% \\
100.0 \% \\
100.0 \% \\
100.0 \% \\
100.0 \% \\
100.0 \% \\
59.0 \% \\
100.0 \% \\
100.0 \% \\
100.0 \% \\
100.0 \% \\
100.0 \%\end{array}$ & $\begin{array}{l}13,723 \\
16,137 \\
62,109 \\
31,704 \\
25,764 \\
30,796 \\
15,593 \\
51,703 \\
99,126 \\
45,377 \\
74,291 \\
25,357 \\
17,150 \\
54,173 \\
76401 \\
7,219\end{array}$ \\
\hline Total Ghazni & & 30,459 & $4.7 \%$ & 616,164 & $95.3 \%$ & 646,623 \\
\hline Ghor & $\begin{array}{l}\text { Cheghcheran } \\
\text { Ghore Taywara } \\
\text { Lalwasarjanga } \\
\text { Pasaband } \\
\text { Saghar } \\
\text { Shahrak } \\
\text { Toolak }\end{array}$ & $\begin{array}{r}2,947 \\
0 \\
0 \\
0 \\
0 \\
0 \\
0\end{array}$ & $\begin{array}{l}3.3 \% \\
0.0 \% \\
0.0 \% \\
0.0 \% \\
0.0 \% \\
0.0 \% \\
0.0 \%\end{array}$ & $\begin{array}{l}86,356 \\
48,488 \\
65,548 \\
45,797 \\
18,822 \\
41,674 \\
28,360\end{array}$ & $\begin{array}{r}96.7 \% \\
100.0 \% \\
100.0 \% \\
100.0 \% \\
100.0 \% \\
100.0 \% \\
100.0 \%\end{array}$ & $\begin{array}{l}89,303 \\
48,488 \\
65,548 \\
45,797 \\
18,822 \\
41,674 \\
28,360\end{array}$ \\
\hline Total Ghor & & 2,947 & $0.9 \%$ & 335,045 & $99.1 \%$ & 337,992 \\
\hline Helmand & $\begin{array}{l}\text { Baghran } \\
\text { Bust } \\
\text { Deshu } \\
\text { Garmser } \\
\text { Kajaki } \\
\text { Khanishan } \\
\text { Mousa Qala } \\
\text { Nadi Ali } \\
\text { Nahre Saraj } \\
\text { Nawaibaregzai } \\
\text { Nawzad } \\
\text { Washir }\end{array}$ & $\begin{array}{r}0 \\
21,621 \\
0 \\
0 \\
0 \\
0 \\
0 \\
0 \\
5,035 \\
0 \\
0 \\
0\end{array}$ & $\begin{array}{r}0.0 \% \\
38.4 \% \\
0.0 \% \\
0.0 \% \\
0.0 \% \\
0.0 \% \\
0.0 \% \\
0.0 \% \\
7.2 \% \\
0.0 \% \\
0.0 \% \\
0.0 \%\end{array}$ & $\begin{array}{r}47,003 \\
34,684 \\
11,806 \\
50,684 \\
69,647 \\
15,167 \\
37,119 \\
68,121 \\
64,897 \\
53,581 \\
29,324 \\
8,956\end{array}$ & $\begin{array}{r}100.0 \% \\
61.6 \% \\
100.0 \% \\
100.0 \% \\
100.0 \% \\
100.0 \% \\
100.0 \% \\
100.0 \% \\
92.8 \% \\
100.0 \% \\
100.0 \% \\
100.0 \%\end{array}$ & $\begin{array}{r}47,003 \\
56,305 \\
11,806 \\
50,684 \\
69,647 \\
15,167 \\
37,119 \\
68,121 \\
69,932 \\
53,581 \\
29,324 \\
8,956\end{array}$ \\
\hline Total Helmand & & 26,656 & $5.1 \%$ & 490,989 & $94.9 \%$ & 517,645 \\
\hline Herat & $\begin{array}{l}\text { Adraskan } \\
\text { Anjil } \\
\text { Cheshtisharif } \\
\text { Farsi } \\
\text { Ghuryan }\end{array}$ & $\begin{array}{r}0 \\
0 \\
0 \\
0 \\
12,416\end{array}$ & $\begin{array}{r}0.0 \% \\
0.0 \% \\
0.0 \% \\
0.0 \% \\
34.4 \%\end{array}$ & $\begin{array}{r}22,893 \\
137,483 \\
9,785 \\
15,908 \\
23,678\end{array}$ & $\begin{array}{r}100.0 \% \\
100.0 \% \\
100.0 \% \\
100.0 \% \\
65.6 \%\end{array}$ & $\begin{array}{r}22,893 \\
137,483 \\
9,785 \\
15,908 \\
36,094\end{array}$ \\
\hline
\end{tabular}


Table A VI-6 (cont.). Official Census Population Estimates for Afghanistan by Province and District, 1978/79

\begin{tabular}{|c|c|c|c|c|c|c|}
\hline Province & Woliswali & Urban & $\%$ & Rural & $\%$ & Total \\
\hline Herat (cont.) & $\begin{array}{l}\text { Gozara } \\
\text { Karukh } \\
\text { Kohsan } \\
\text { Koshk } \\
\text { Markiz Herat } \\
\text { Obe } \\
\text { Pashtunzarghu } \\
\text { Shindand } \\
\text { Zendajan }\end{array}$ & $\begin{array}{r}0 \\
5,399 \\
0 \\
0 \\
140,323 \\
1,505 \\
0 \\
4,300 \\
0\end{array}$ & $\begin{array}{r}0.0 \% \\
16.8 \% \\
0.0 \% \\
0.0 \% \\
100.0 \% \\
4.3 \% \\
0.0 \% \\
5.6 \% \\
0.0 \%\end{array}$ & $\begin{array}{r}76,359 \\
26,740 \\
22,577 \\
48,929 \\
0 \\
33,486 \\
53,195 \\
72,480 \\
27,884\end{array}$ & $\begin{array}{r}100.0 \% \\
83.2 \% \\
100.0 \% \\
100.0 \% \\
0.0 \% \\
95.7 \% \\
100.0 \% \\
94.4 \% \\
100.0 \%\end{array}$ & $\begin{array}{r}76,359 \\
32,139 \\
22,577 \\
48,929 \\
140,323 \\
34,991 \\
53,195 \\
76,780 \\
27,884\end{array}$ \\
\hline Total Herat & & 163,943 & $22.3 \%$ & 571,397 & $77.7 \%$ & 735,340 \\
\hline Jowzjan & $\begin{array}{l}\text { Aqcha } \\
\text { Balkhab } \\
\text { Faizabad } \\
\text { Khamyab } \\
\text { Kohistanat } \\
\text { Mangajek } \\
\text { Mardyan } \\
\text { Qarqin } \\
\text { Sange Charak } \\
\text { Saripul } \\
\text { Sheberghan }\end{array}$ & $\begin{array}{r}8,906 \\
0 \\
0 \\
0 \\
0 \\
0 \\
0 \\
3,561 \\
7,718 \\
15,675 \\
18,960\end{array}$ & $\begin{array}{r}14.3 \% \\
0.0 \% \\
0.0 \% \\
0.0 \% \\
0.0 \% \\
0.0 \% \\
0.0 \% \\
25.4 \% \\
6.1 \% \\
12.3 \% \\
18.8 \%\end{array}$ & $\begin{array}{r}53,376 \\
27,871 \\
24,754 \\
7,344 \\
42,728 \\
28,127 \\
26,702 \\
10,459 \\
118,811 \\
111,765 \\
81,892\end{array}$ & $\begin{array}{r}85.7 \% \\
100.0 \% \\
100.0 \% \\
100.0 \% \\
100.0 \% \\
100.0 \% \\
100.0 \% \\
74.6 \% \\
93.9 \% \\
87.7 \% \\
81.2 \%\end{array}$ & $\begin{array}{r}62,282 \\
27,871 \\
24,754 \\
7,344 \\
42,728 \\
28,127 \\
26,702 \\
14,020 \\
126,529 \\
127,440 \\
100,852\end{array}$ \\
\hline Total Jowzjan & & 54,821 & $9.3 \%$ & 533,828 & $90.7 \%$ & 588,649 \\
\hline Kabul & $\begin{array}{l}\text { Bagrami } \\
\text { Charasyab } \\
\text { Deh Sabz } \\
\text { Istalif } \\
\text { Kabul City } \\
\text { Kalakan } \\
\text { Khake Jabar } \\
\text { Mir Bacha Kot } \\
\text { Paghman } \\
\text { Sarobi } \\
\text { Shakar Dara }\end{array}$ & $\begin{array}{r}0 \\
0 \\
0 \\
0 \\
913,164 \\
0 \\
0 \\
0 \\
0 \\
0 \\
0\end{array}$ & $\begin{array}{r}0.0 \% \\
0.0 \% \\
0.0 \% \\
0.0 \% \\
100.0 \% \\
0.0 \% \\
0.0 \% \\
0.0 \% \\
0.0 \% \\
0.0 \% \\
0.0 \%\end{array}$ & $\begin{array}{r}41,515 \\
55,008 \\
30,098 \\
29,655 \\
0 \\
22,736 \\
11,418 \\
50,856 \\
59,130 \\
33,370 \\
72,652\end{array}$ & $\begin{array}{r}100.0 \% \\
100.0 \% \\
100.0 \% \\
100.0 \% \\
0.0 \% \\
100.0 \% \\
100.0 \% \\
100.0 \% \\
100.0 \% \\
100.0 \% \\
100.0 \%\end{array}$ & $\begin{array}{r}41,515 \\
55,008 \\
30,098 \\
29,655 \\
913,164 \\
22,736 \\
11,418 \\
50,856 \\
59,130 \\
33,370 \\
72,652\end{array}$ \\
\hline Total Kabul & & 913,164 & $69.2 \%$ & 406,438 & $30.8 \%$ & $1,319,602$ \\
\hline Kandahar & $\begin{array}{l}\text { Arghandab } \\
\text { Arghistan } \\
\text { Daman } \\
\text { Dehla } \\
\text { Ghorak } \\
\text { Khakraiz } \\
\text { Markizkandaha } \\
\text { Maruf } \\
\text { Maywand } \\
\text { Panjwai } \\
\text { Rek } \\
\text { Shorabak } \\
\text { Spin Boldak }\end{array}$ & $\begin{array}{r}0 \\
0 \\
0 \\
0 \\
0 \\
0 \\
178,438 \\
0 \\
0 \\
0 \\
0 \\
0 \\
0\end{array}$ & $\begin{array}{r}0.0 \% \\
0.0 \% \\
0.0 \% \\
0.0 \% \\
0.0 \% \\
0.0 \% \\
64.3 \% \\
0.0 \% \\
0.0 \% \\
0.0 \% \\
0.0 \% \\
0.0 \% \\
0.0 \%\end{array}$ & $\begin{array}{r}43,047 \\
19,868 \\
17,005 \\
24,966 \\
5,514 \\
13,166 \\
99,070 \\
19,040 \\
38,559 \\
72,666 \\
1,103 \\
6,616 \\
28,146\end{array}$ & $\begin{array}{r}100.0 \% \\
100.0 \% \\
100.0 \% \\
100.0 \% \\
100.0 \% \\
100.0 \% \\
35.7 \% \\
100.0 \% \\
100.0 \% \\
100.0 \% \\
100.0 \% \\
100.0 \% \\
100.0 \%\end{array}$ & $\begin{array}{r}43,047 \\
19,868 \\
17,005 \\
24,966 \\
5,514 \\
13,166 \\
277,508 \\
19,040 \\
38,559 \\
72,666 \\
1,103 \\
6,616 \\
28,146\end{array}$ \\
\hline Total Kandahar & & 178,438 & $31.5 \%$ & 388,766 & $68.5 \%$ & 567,204 \\
\hline Kapisa & $\begin{array}{l}\text { Alasai } \\
\text { Kohi Band } \\
\text { Kohistan } \\
\text { Mahmood Raqi } \\
\text { Nejrab } \\
\text { Panjsher } \\
\text { Panjsher } 1 \\
\text { Panjsher } 2 \\
\text { Tagab }\end{array}$ & $\begin{array}{r}0 \\
0 \\
0 \\
1,215 \\
0 \\
0 \\
0 \\
0 \\
0\end{array}$ & $\begin{array}{l}0.0 \% \\
0.0 \% \\
0.0 \% \\
3.0 \% \\
0.0 \% \\
0.0 \% \\
0.0 \% \\
0.0 \% \\
0.0 \%\end{array}$ & $\begin{array}{l}19,292 \\
13,504 \\
69,000 \\
39,298 \\
65,802 \\
47,661 \\
32,741 \\
14,820 \\
42,442\end{array}$ & $\begin{array}{r}100.0 \% \\
100.0 \% \\
100.0 \% \\
97.0 \% \\
100.0 \% \\
100.0 \% \\
100.0 \% \\
100.0 \% \\
100.0 \%\end{array}$ & $\begin{array}{l}19,292 \\
13,504 \\
69,000 \\
40,513 \\
65,802 \\
47,661 \\
32,741 \\
14,820 \\
42,442\end{array}$ \\
\hline Total Kapisa & & 1,215 & $0.4 \%$ & 344,560 & $99.6 \%$ & 345,775 \\
\hline
\end{tabular}


Table A VI-6 (cont.). Official Census Population Estimates for Afghanistan by Province and District, 1978/79

\begin{tabular}{|c|c|c|c|c|c|c|}
\hline Province & Woliswali & Urban & $\%$ & Rural & $\%$ & Total \\
\hline Kunar & $\begin{array}{l}\text { Asadabad } \\
\text { Bar Kunar } \\
\text { Bargey Matal } \\
\text { Chapa Dara } \\
\text { Chowki } \\
\text { Dangam } \\
\text { Kamdesh } \\
\text { Khas Kunar } \\
\text { Marawar } \\
\text { Narang } \\
\text { Naray } \\
\text { Nourgal } \\
\text { Peche } \\
\text { Sir Kanay }\end{array}$ & $\begin{array}{r}2,097 \\
0 \\
0 \\
0 \\
0 \\
0 \\
0 \\
0 \\
0 \\
0 \\
0 \\
0 \\
0 \\
0\end{array}$ & $\begin{array}{l}7.2 \% \\
0.0 \% \\
0.0 \% \\
0.0 \% \\
0.0 \% \\
0.0 \% \\
0.0 \% \\
0.0 \% \\
0.0 \% \\
0.0 \% \\
0.0 \% \\
0.0 \% \\
0.0 \% \\
0.0 \%\end{array}$ & $\begin{array}{r}27,023 \\
25,504 \\
5,885 \\
21,683 \\
21,580 \\
8,870 \\
9,199 \\
18,586 \\
11,771 \\
15,698 \\
9,467 \\
19,386 \\
38,236 \\
15,147\end{array}$ & $\begin{array}{r}92.8 \% \\
100.0 \% \\
100.0 \% \\
100.0 \% \\
100.0 \% \\
100.0 \% \\
100.0 \% \\
100.0 \% \\
100.0 \% \\
100.0 \% \\
100.0 \% \\
100.0 \% \\
100.0 \% \\
100.0 \%\end{array}$ & $\begin{array}{r}29,120 \\
25,504 \\
5,885 \\
21,683 \\
21,580 \\
8,870 \\
9,199 \\
18,586 \\
11,771 \\
15,698 \\
9,467 \\
19,386 \\
38,236 \\
15,147\end{array}$ \\
\hline Total Kunar & & 2,097 & $0.8 \%$ & 248,035 & $99.2 \%$ & 250,132 \\
\hline Kunduz & $\begin{array}{l}\text { Aliabad } \\
\text { Archi } \\
\text { Char Dara } \\
\text { Hazrat Imam } \\
\text { Khanabad } \\
\text { Markiz Kunduz } \\
\text { Qalae Zal }\end{array}$ & $\begin{array}{r}0 \\
2,873 \\
0 \\
12,646 \\
26,827 \\
53,199 \\
11,621\end{array}$ & $\begin{array}{r}0.0 \% \\
4.9 \% \\
0.0 \% \\
10.0 \% \\
26.2 \% \\
33.7 \% \\
29.0 \%\end{array}$ & $\begin{array}{r}27,892 \\
55,760 \\
42,127 \\
113,811 \\
75,567 \\
104,662 \\
28,452\end{array}$ & $\begin{array}{r}100.0 \% \\
95.1 \% \\
100.0 \% \\
90.0 \% \\
73.8 \% \\
66.3 \% \\
71.0 \%\end{array}$ & $\begin{array}{r}27,892 \\
58,633 \\
42,127 \\
126,457 \\
102,394 \\
157,861 \\
40,073\end{array}$ \\
\hline Total Kunduz & & 107,166 & $19.3 \%$ & 448,271 & $80.7 \%$ & 555,437 \\
\hline Laghman & $\begin{array}{l}\text { Ali Sheng } \\
\text { Alinegar } \\
\text { Dowlat Shah } \\
\text { Mehterlam } \\
\text { Norestan } \\
\text { Qarghaie }\end{array}$ & $\begin{array}{r}0 \\
0 \\
0 \\
4,012 \\
0 \\
0\end{array}$ & $\begin{array}{l}0.0 \% \\
0.0 \% \\
0.0 \% \\
5.0 \% \\
0.0 \% \\
0.0 \%\end{array}$ & $\begin{array}{l}42,337 \\
50,177 \\
21,663 \\
76,222 \\
51,350 \\
64,990\end{array}$ & $\begin{array}{r}100.0 \% \\
100.0 \% \\
100.0 \% \\
95.0 \% \\
100.0 \% \\
100.0 \%\end{array}$ & $\begin{array}{l}42,337 \\
50,177 \\
21,663 \\
80,234 \\
51,350 \\
64,990\end{array}$ \\
\hline Total Laghman & & 4,012 & $1.3 \%$ & 306,739 & $98.7 \%$ & 310,751 \\
\hline Logar & $\begin{array}{l}\text { Azro } \\
\text { Baraki } \\
\text { Charkh } \\
\text { Kolangar } \\
\text { Mohammad Agha }\end{array}$ & $\begin{array}{r}0 \\
0 \\
0 \\
3,870 \\
0\end{array}$ & $\begin{array}{l}0.0 \% \\
0.0 \% \\
0.0 \% \\
5.0 \% \\
0.0 \%\end{array}$ & $\begin{array}{l}13,480 \\
44,186 \\
34,598 \\
73,521 \\
46,649\end{array}$ & $\begin{array}{r}100.0 \% \\
100.0 \% \\
100.0 \% \\
95.0 \% \\
100.0 \%\end{array}$ & $\begin{array}{l}13,480 \\
44,186 \\
34,598 \\
77,390 \\
46,649\end{array}$ \\
\hline Total Logar & & 3,870 & $1.8 \%$ & 212,434 & $98.2 \%$ & 216,303 \\
\hline Nangarhar & $\begin{array}{l}\text { Achin } \\
\text { Bati Kot } \\
\text { Chaparhar } \\
\text { Darae Noor } \\
\text { Deh Bala } \\
\text { Dorbaba } \\
\text { Goshta } \\
\text { Hesarak } \\
\text { Jalalabad } \\
\text { Kama } \\
\text { Khogiani } \\
\text { Kouz Kunar } \\
\text { Lalpur } \\
\text { Mohmand Dara } \\
\text { Nazian } \\
\text { Pachier } \\
\text { Rodat } \\
\text { Sher Zad } \\
\text { Shinwar } \\
\text { Sorkhroad }\end{array}$ & $\begin{array}{r}0 \\
0 \\
0 \\
0 \\
0 \\
0 \\
0 \\
0 \\
53,894 \\
0 \\
2,443 \\
0 \\
0 \\
0 \\
0 \\
0 \\
0 \\
0 \\
0 \\
0\end{array}$ & $\begin{array}{r}0.0 \% \\
0.0 \% \\
0.0 \% \\
0.0 \% \\
0.0 \% \\
0.0 \% \\
0.0 \% \\
0.0 \% \\
47.4 \% \\
0.0 \% \\
3.3 \% \\
0.0 \% \\
0.0 \% \\
0.0 \% \\
0.0 \% \\
0.0 \% \\
0.0 \% \\
0.0 \% \\
0.0 \% \\
0.0 \%\end{array}$ & $\begin{array}{r}50,304 \\
38,914 \\
30,372 \\
27,606 \\
23,728 \\
17,762 \\
9,991 \\
16,135 \\
59,806 \\
53,151 \\
71,589 \\
34,414 \\
10,440 \\
22,869 \\
5,551 \\
21,803 \\
60,813 \\
33,236 \\
24,423 \\
76,742 \\
\end{array}$ & $\begin{array}{r}100.0 \% \\
100.0 \% \\
100.0 \% \\
100.0 \% \\
100.0 \% \\
100.0 \% \\
100.0 \% \\
100.0 \% \\
52.6 \% \\
100.0 \% \\
96.7 \% \\
100.0 \% \\
100.0 \% \\
100.0 \% \\
100.0 \% \\
100.0 \% \\
100.0 \% \\
100.0 \% \\
100.0 \% \\
100.0 \%\end{array}$ & $\begin{array}{r}50,304 \\
38,914 \\
30,372 \\
27,606 \\
23,728 \\
17,762 \\
9,991 \\
16,135 \\
113,700 \\
53,151 \\
74,032 \\
34,414 \\
10,440 \\
22,869 \\
5,551 \\
21,803 \\
60,813 \\
33,236 \\
24,423 \\
76,742\end{array}$ \\
\hline Total Nangarhar & & 56,337 & $7.6 \%$ & 689,649 & $92.4 \%$ & 745,986 \\
\hline
\end{tabular}


Table A VI-6 (cont.). Official Census Population Estimates for Afghanistan by Province and District, 1978/79

\begin{tabular}{|c|c|c|c|c|c|c|}
\hline Province & Woliswali & Urban & $\%$ & Rural & $\%$ & Total \\
\hline Nimroz & $\begin{array}{l}\text { Aslechakhansr } \\
\text { Charborjak } \\
\text { Kang } \\
\text { Khash Raod } \\
\text { Zaranj }\end{array}$ & $\begin{array}{r}0 \\
0 \\
0 \\
0 \\
6,419\end{array}$ & $\begin{array}{r}0.0 \% \\
0.0 \% \\
0.0 \% \\
0.0 \% \\
22.0 \%\end{array}$ & $\begin{array}{l}19,864 \\
24,983 \\
16,655 \\
12,954 \\
22,759\end{array}$ & $\begin{array}{c}100.0 \% \\
100.0 \% \\
100.0 \% \\
100.0 \% \\
78.0 \%\end{array}$ & $\begin{array}{l}19,864 \\
24,983 \\
16,655 \\
12,954 \\
29,178\end{array}$ \\
\hline Total Nimroz & & 6,419 & $6.2 \%$ & 97,215 & $93.8 \%$ & 103,634 \\
\hline Paktia & $\begin{array}{l}\text { Bak } \\
\text { Chamkani } \\
\text { Dand Wa Patan } \\
\text { Gardez } \\
\text { Gurboz } \\
\text { Jadran } \\
\text { Jaji } \\
\text { Jaji Maidan } \\
\text { Jani Khil } \\
\text { Khost } \\
\text { Lajmangal } \\
\text { Mandozai } \\
\text { Mousa Khil } \\
\text { Nader Shahkot } \\
\text { Qalander } \\
\text { Saroti } \\
\text { Sayed Karam } \\
\text { Shamal } \\
\text { Shawak } \\
\text { Sperah } \\
\text { Tani } \\
\text { Trizai } \\
\text { Zormat }\end{array}$ & $\begin{array}{r}0 \\
0 \\
0 \\
9,567 \\
0 \\
0 \\
0 \\
0 \\
0 \\
1,866 \\
0 \\
0 \\
0 \\
0 \\
0 \\
0 \\
0 \\
0 \\
0 \\
0 \\
0 \\
0 \\
0\end{array}$ & $\begin{array}{r}0.0 \% \\
0.0 \% \\
0.0 \% \\
21.6 \% \\
0.0 \% \\
0.0 \% \\
0.0 \% \\
0.0 \% \\
0.0 \% \\
3.8 \% \\
0.0 \% \\
0.0 \% \\
0.0 \% \\
0.0 \% \\
0.0 \% \\
0.0 \% \\
0.0 \% \\
0.0 \% \\
0.0 \% \\
0.0 \% \\
0.0 \% \\
0.0 \% \\
0.0 \%\end{array}$ & $\begin{array}{r}6,819 \\
17,332 \\
10,081 \\
34,725 \\
9,376 \\
15,026 \\
30,812 \\
12,150 \\
12,517 \\
47,240 \\
16,369 \\
23,109 \\
18,932 \\
11,554 \\
4,150 \\
20,917 \\
52,435 \\
5,853 \\
5,016 \\
10,592 \\
23,109 \\
19,257 \\
65,219\end{array}$ & $\begin{array}{r}100.0 \% \\
100.0 \% \\
100.0 \% \\
78.4 \% \\
100.0 \% \\
100.0 \% \\
100.0 \% \\
100.0 \% \\
100.0 \% \\
96.2 \% \\
100.0 \% \\
100.0 \% \\
100.0 \% \\
100.0 \% \\
100.0 \% \\
100.0 \% \\
100.0 \% \\
100.0 \% \\
100.0 \% \\
100.0 \% \\
100.0 \% \\
100.0 \% \\
100.0 \%\end{array}$ & $\begin{array}{r}6,819 \\
17,332 \\
10,081 \\
44,292 \\
9,376 \\
15,026 \\
30,812 \\
12,150 \\
12,517 \\
49,106 \\
16,369 \\
23,109 \\
18,932 \\
11,554 \\
4,150 \\
20,917 \\
52,435 \\
5,853 \\
5,016 \\
10,592 \\
23,109 \\
19,257 \\
65,219\end{array}$ \\
\hline Total Paktia & & 11,433 & $2.4 \%$ & 472,590 & $97.6 \%$ & 484,023 \\
\hline Paktika & $\begin{array}{l}\text { Barmal } \\
\text { Dila } \\
\text { Gayan } \\
\text { Gomal } \\
\text { Katawaz } \\
\text { Mata Khan } \\
\text { Neka } \\
\text { Omna } \\
\text { Sar Rawza } \\
\text { Sarobi } \\
\text { Sharan } \\
\text { Urgoun } \\
\text { Walmamy } \\
\text { Wazakhwa } \\
\text { Zelook }\end{array}$ & $\begin{array}{r}0 \\
0 \\
0 \\
0 \\
0 \\
0 \\
0 \\
0 \\
0 \\
0 \\
1,388 \\
0 \\
0 \\
0 \\
0\end{array}$ & $\begin{array}{l}0.0 \% \\
0.0 \% \\
0.0 \% \\
0.0 \% \\
0.0 \% \\
0.0 \% \\
0.0 \% \\
0.0 \% \\
0.0 \% \\
0.0 \% \\
3.8 \% \\
0.0 \% \\
0.0 \% \\
0.0 \% \\
0.0 \%\end{array}$ & $\begin{array}{r}20,064 \\
14,491 \\
20,064 \\
4,459 \\
41,042 \\
14,364 \\
7,182 \\
6,990 \\
13,376 \\
7,182 \\
35,129 \\
31,808 \\
2,052 \\
14,491 \\
11,147\end{array}$ & $\begin{array}{r}100.0 \% \\
100.0 \% \\
100.0 \% \\
100.0 \% \\
100.0 \% \\
100.0 \% \\
100.0 \% \\
100.0 \% \\
100.0 \% \\
100.0 \% \\
96.2 \% \\
100.0 \% \\
100.0 \% \\
100.0 \% \\
100.0 \%\end{array}$ & $\begin{array}{r}20,064 \\
14,491 \\
20,064 \\
4,459 \\
41,042 \\
14,364 \\
7,182 \\
6,990 \\
13,376 \\
7,182 \\
36,517 \\
31,808 \\
2,052 \\
14,491 \\
11,147 \\
-0-0 \\
\end{array}$ \\
\hline Total Paktika & & 1,388 & $0.6 \%$ & 243,841 & $99.4 \%$ & 245,229 \\
\hline Parwan & $\begin{array}{l}\text { Bagram } \\
\text { Chari Kar } \\
\text { Ghorband } \\
\text { Jabul Saraj } \\
\text { Kohe Safi } \\
\text { Salang } \\
\text { Shekh Ali } \\
\text { Shinwar } \\
\text { Surkhi Parsa }\end{array}$ & $\begin{array}{r}0 \\
22,473 \\
0 \\
1,336 \\
0 \\
0 \\
0 \\
0 \\
0\end{array}$ & $\begin{array}{r}0.0 \% \\
20.8 \% \\
0.0 \% \\
1.8 \% \\
0.0 \% \\
0.0 \% \\
0.0 \% \\
0.0 \% \\
0.0 \%\end{array}$ & $\begin{array}{l}68,010 \\
85,572 \\
60,711 \\
72,872 \\
11,721 \\
15,434 \\
19,292 \\
20,257 \\
31,832\end{array}$ & $\begin{array}{r}100.0 \% \\
79.2 \% \\
100.0 \% \\
98.2 \% \\
100.0 \% \\
100.0 \% \\
100.0 \% \\
100.0 \% \\
100.0 \%\end{array}$ & $\begin{array}{r}68,010 \\
108,045 \\
60,711 \\
74,208 \\
11,721 \\
15,434 \\
19,292 \\
20,257 \\
31,832\end{array}$ \\
\hline Total Parwan & & 23,809 & $5.8 \%$ & 385,701 & $94.2 \%$ & 409,510 \\
\hline Samangan & $\begin{array}{l}\text { Darae Souf } \\
\text { Hazratesultan } \\
\text { Kaldar }\end{array}$ & $\begin{array}{l}0 \\
0 \\
0\end{array}$ & $\begin{array}{l}0.0 \% \\
0.0 \% \\
0.0 \%\end{array}$ & $\begin{array}{l}82,535 \\
23,690 \\
10,891\end{array}$ & $\begin{array}{l}100.0 \% \\
100.0 \% \\
100.0 \%\end{array}$ & $\begin{array}{l}82,535 \\
23,690 \\
10,891\end{array}$ \\
\hline
\end{tabular}


Table A VI-6 (cont.). Official Census Population Estimates for Afghanistan by Province and District, $1978 / 79$

\begin{tabular}{|c|c|c|c|c|c|c|}
\hline Province & Woliswali & Urban & $\%$ & Rural & $\%$ & Total \\
\hline Samangan (cont.) & $\begin{array}{l}\text { Khuim } \\
\text { Markizsamanga } \\
\text { Roye Doab }\end{array}$ & $\begin{array}{r}28,054 \\
4,924 \\
0\end{array}$ & $\begin{array}{r}55.7 \% \\
6.1 \% \\
0.0 \%\end{array}$ & $\begin{array}{l}22,313 \\
75,793 \\
24,384\end{array}$ & $\begin{array}{r}44.3 \% \\
93.9 \% \\
100.0 \%\end{array}$ & $\begin{array}{l}50,367 \\
80,717 \\
24,384\end{array}$ \\
\hline Total Samangan & & 32,978 & $12.1 \%$ & 239,606 & $87.9 \%$ & 272,584 \\
\hline Takhar & $\begin{array}{l}\text { Bangi } \\
\text { Cha Ab } \\
\text { Chal } \\
\text { Darqad } \\
\text { Eshkamesh } \\
\text { Farkhar } \\
\text { Kalafgan } \\
\text { Khwaja Ghar } \\
\text { Rustaq } \\
\text { Taloqan } \\
\text { Warsaj } \\
\text { Yangi Qala }\end{array}$ & $\begin{array}{r}0 \\
10,821 \\
0 \\
0 \\
0 \\
1,286 \\
0 \\
3,458 \\
6,585 \\
19,945 \\
0 \\
4,066\end{array}$ & $\begin{array}{r}0.0 \% \\
43.6 \% \\
0.0 \% \\
0.0 \% \\
0.0 \% \\
4.7 \% \\
0.0 \% \\
6.0 \% \\
6.8 \% \\
14.4 \% \\
0.0 \% \\
13.1 \%\end{array}$ & $\begin{array}{r}21,316 \\
13,997 \\
15,500 \\
13,036 \\
32,656 \\
26,069 \\
21,396 \\
54,173 \\
90,259 \\
118,565 \\
20,555 \\
26,969\end{array}$ & $\begin{array}{r}100.0 \% \\
56.4 \% \\
100.0 \% \\
100.0 \% \\
100.0 \% \\
95.3 \% \\
100.0 \% \\
94.0 \% \\
93.2 \% \\
85.6 \% \\
100.0 \% \\
86.9 \%\end{array}$ & $\begin{array}{r}21,316 \\
24,818 \\
15,500 \\
13,036 \\
32,656 \\
27,355 \\
21,396 \\
57,631 \\
96,844 \\
138,510 \\
20,555 \\
31,035\end{array}$ \\
\hline Total Takhar & & 46,161 & $9.2 \%$ & 454,491 & $90.8 \%$ & 500,652 \\
\hline Uruzgan & $\begin{array}{l}\text { Ajristan } \\
\text { Chorah } \\
\text { Dai Kundi } \\
\text { Deh Raod } \\
\text { Gezab } \\
\text { Kajran } \\
\text { Khas Uruzgan } \\
\text { Sharistan } \\
\text { Tirin Kot }\end{array}$ & $\begin{array}{r}0 \\
0 \\
0 \\
0 \\
1,284 \\
1,808 \\
0 \quad 0 \\
0 \\
0 \\
3,348\end{array}$ & $\begin{array}{l}0.0 \% \\
0.0 \% \\
0.0 \% \\
3.6 \% \\
4.8 \% \\
0.0 \% \\
0.0 \% \\
0.0 \% \\
7.7 \%\end{array}$ & $\begin{array}{r}35,000 \\
24,042 \\
106,900 \\
34,393 \\
35,860 \\
39,371 \\
39,465 \\
74,819 \\
40,128\end{array}$ & $\begin{array}{r}100.0 \% \\
100.0 \% \\
100.0 \% \\
96.4 \% \\
95.2 \% \\
100.0 \% \\
100.0 \% \\
100.0 \% \\
92.3 \%\end{array}$ & $\begin{array}{r}35,000 \\
24,042 \\
106,900 \\
35,677 \\
37,668 \\
39,371 \\
39,465 \\
74,819 \\
43,476\end{array}$ \\
\hline Total Uruzgan & & 6,440 & $1.5 \%$ & 429,978 & $98.5 \%$ & 436,418 \\
\hline Wardak & $\begin{array}{l}\text { Behsud } \\
\text { Chaki Wardak } \\
\text { Day Mirdad } \\
\text { Maidan } \\
\text { Markazebehsud } \\
\text { Sayed Abad }\end{array}$ & $\begin{array}{r}0 \\
0 \\
0 \\
2,056 \\
0 \\
0\end{array}$ & $\begin{array}{l}0.0 \% \\
0.0 \% \\
0.0 \% \\
2.6 \% \\
0.0 \% \\
0.0 \%\end{array}$ & $\begin{array}{l}25,701 \\
39,489 \\
15,816 \\
77,025 \\
73,150 \\
54,368\end{array}$ & $\begin{array}{r}100.0 \% \\
100.0 \% \\
100.0 \% \\
97.4 \% \\
100.0 \% \\
100.0 \%\end{array}$ & $\begin{array}{l}25,701 \\
39,489 \\
15,816 \\
79,081 \\
73,150 \\
54,368\end{array}$ \\
\hline Total Wardak & & 2,056 & $0.7 \%$ & 285,549 & $99.3 \%$ & 287,605 \\
\hline Zabul & $\begin{array}{l}\text { Arghandab } \\
\text { Atghar } \\
\text { Dai Chopan } \\
\text { Jaldak } \\
\text { Mizan } \\
\text { Qalat } \\
\text { Shahjoy } \\
\text { Shamaizai } \\
\text { Shinkai }\end{array}$ & $\begin{array}{r}0 \\
0 \\
0 \\
0 \\
0 \\
5,953 \\
0 \\
0 \\
0\end{array}$ & $\begin{array}{r}0.0 \% \\
0.0 \% \\
0.0 \% \\
0.0 \% \\
0.0 \% \\
30.5 \% \\
0.0 \% \\
0.0 \% \\
0.0 \%\end{array}$ & $\begin{array}{r}18,821 \\
5,006 \\
45,225 \\
9,884 \\
7,878 \\
13,565 \\
33,716 \\
25,763 \\
13,551\end{array}$ & $\begin{array}{r}100.0 \% \\
100.0 \% \\
100.0 \% \\
100.0 \% \\
100.0 \% \\
69.5 \% \\
100.0 \% \\
100.0 \% \\
100.0 \%\end{array}$ & $\begin{array}{r}18,821 \\
5,006 \\
45,225 \\
9,884 \\
7,878 \\
19,518 \\
33,716 \\
25,763 \\
13,551\end{array}$ \\
\hline Total Zabul & & 5,953 & $3.3 \%$ & 173,409 & $96.7 \%$ & 179,362 \\
\hline Total, Afghanistan & & $1,874,796$ & $14.5 \%$ & $11,091,505$ & $85.5 \%$ & $12,966,301$ \\
\hline
\end{tabular}

Note: These figures are taken from a draft report prepared by Dr. Richard English for the UNHCR and presented to the RRNA research team in June 1989. Certain minor districts are apparently missing from the series, for which reason the total population sums to slightly less than the usually quoted official figure of $13,051,358$. Absolute figures for rural and urban populations are calculated from percentages.

Source: Government of Afghanistan, $1978 / 79$ Census; cited from UNHCR. 
Table A VI-7. World Bank Series on Population and Rates of Growth

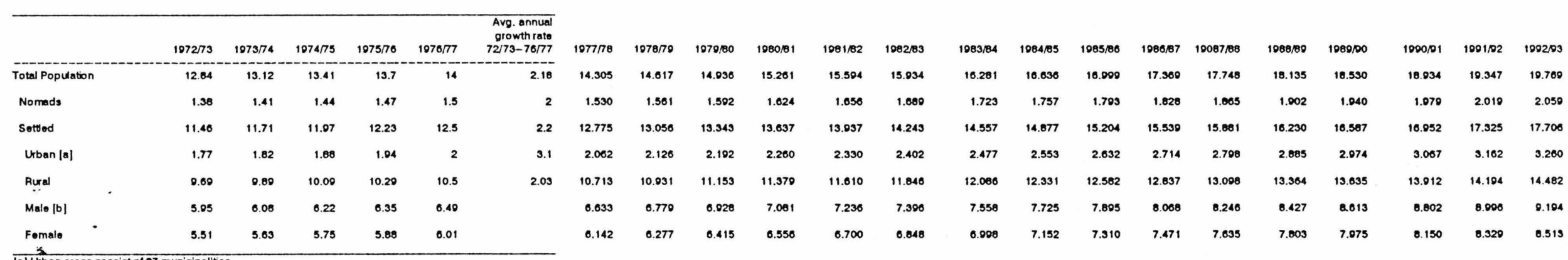

[a] Utban areas consist of 87 municipalitios

(b) Assuming a sox-rat
ADS which is too Nigh.

Souros: Atghan Demographic Survey, CSO, and World Bank Mission estimatess; dited
trom 'A Aghanistan: The Journey to Economic Development, Vol. II, March 1078,
World Bank. 
Table A VI-7. World Bank Series on Population and Rates of Growth

\begin{tabular}{lrrrrrr}
\hline & & & & & $\begin{array}{r}\text { Avg. annual } \\
\text { growth rate } \\
72 / 73-76 / 77\end{array}$ \\
\hline Total Population & $1972 / 73$ & $1973 / 74$ & $1974 / 75$ & $1975 / 76$ & $1976 / 77$ & 2.18 \\
Nomads & 12.84 & 13.12 & 13.41 & 13,7 & 14 & 2 \\
Settled & 1.38 & 1.41 & 1.44 & 1.47 & 1.5 & 2.2 \\
Urban [a] & 11.46 & 11.71 & 11.97 & 12.23 & 12.5 & 3.1 \\
Rural & 1.77 & 1.82 & 1.88 & 1.94 & 2 & 2.03 \\
Male [b] & 9.69 & 9.89 & 10.09 & 10.29 & 10.5 & 6.49 \\
Female & 5.95 & 6.08 & 6.22 & 6.35 & 6.01 & \\
\hline
\end{tabular}

[a] Urban areas consist of 87 municipalities.

[b] Assuming a sex-ratio of 108 males/100 females instead of $116 / 100$ given by ADS which is too high.

Source: Afghan Demographic Survey, CSO, and World Bank Mission estimates; cited from "Afghanistan: The Journey to Economic Development", Vol. II, March 1978,

World Bank. 
Table A VI-9. GOA Estimate of Total Population

in Eastern Provinces, 1979 and 1982

\begin{tabular}{|c|c|c|c|c|c|c|}
\hline Province & Rank & 1979 Census & $\begin{array}{r}\% \text { of } \\
\text { Natl Total }\end{array}$ & $1982 \mathrm{CSO}^{*}$ & $\begin{array}{r}\% \text { of } \\
\text { Nat'l Total }\end{array}$ & $\begin{array}{r}\% \text { Increa: } \\
1979-\{\end{array}$ \\
\hline Kabul & 1 & $1,864,000$ & 14.3 & $1,517,909$ & 11.0 & -18.6 \\
\hline Kapisa & 9 & -- & -- & 262,039 & 1.6 & - \\
\hline Parvan & 4 & 755,285 & 5.8 & 527,987 & 3.8 & -30.1 \\
\hline Vardak & 7 & -- & -- & 300,796 & 2.2 & - \\
\hline Lowgar & 11 & -- & -- & 226,234 & 1.6 & - \\
\hline Ghazni & 3 & 646,623 & 5.0 & 676,416 & 4.9 & 4.6 \\
\hline Paktia & 5 & 497,501 & 3.8 & 506,264 & 3.7 & 1.8 \\
\hline Nangarhar & 2 & 745,986 & 5.7 & 781,619 & 5.7 & 4.8 \\
\hline Laghman & 6 & 310,751 & 2.4 & 325,010 & 2.4 & 4.6 \\
\hline Konarha & 10 & 250,132 & 1.9 & 261,604 & 1.9 & 4.6 \\
\hline Bamian & 8 & 268,517 & 2.1 & 280,859 & 2.0 & 4.6 \\
\hline Totals & & $5,338,795$ & 41.0 & $5,666,737$ & 40.8 & 6.1 \\
\hline
\end{tabular}

* Central Statistical Office, Government of Afghanistan.

Orkand Report Note: It is not known how reliable these figures are, though the uniformity in percentages suggests some doctoring of the totals reported.

Sources: 1979 DRA Census: DRA Statistical Yearbook 1360 AH (1981-82); cited from the Orkand Report. 
Table A VI-10. GOA Estimate of Rural Population in Eastern Provinces, 1979 and 1982

\begin{tabular}{|c|c|c|c|c|c|c|}
\hline Province & 1979 Census & $\begin{array}{r}\% \text { of } \\
\text { Prov. Total }\end{array}$ & $\begin{array}{r}\% \text { of } \\
\text { Nat'l Total }\end{array}$ & 1982 CSO & $\begin{array}{r}\% \text { of } \\
\text { Nat'l Total }\end{array}$ & $\begin{array}{r}\% \text { Incr. } \\
1979-82\end{array}$ \\
\hline Kabul & 944,892 & 50.7 & 6.5 & 481,502 & 4.2 & $-49.0 \%$ \\
\hline Kapisa & -- & -- & -- & 260,777 & 2.3 & -- \\
\hline Parvan & 730,192 & 97.0 & 6.6 & 502,870 & 4.3 & $-31.1 \%$ \\
\hline Vardak & -- & -- & -- & 298,643 & 2.6 & -- \\
\hline Lowgar & -- & -- & -- & 220,975 & 1.9 & -- \\
\hline Ghazni & 616,198 & 95.3 & 5.6 & 644,431 & 5.6 & $4.6 \%$ \\
\hline Paktia & 486,086 & 97.8 & 4.4 & 494,263 & 4.3 & $1.7 \%$ \\
\hline Nangarhar & 689,602 & 92.5 & 6.2 & 721,200 & 6.2 & $4.6 \%$ \\
\hline Laghman & 306,764 & 98.7 & 2.8 & 320,819 & 2.8 & $4.6 \%$ \\
\hline Konarha & 248,043 & 99.2 & 2.2 & 259,408 & 2.2 & $4.6 \%$ \\
\hline Bamian & 261,162 & 97.3 & 2.4 & 273,127 & 2.3 & $4.6 \%$ \\
\hline Totals & $4,282,939$ & & 36.7 & $4,478,015$ & 38.7 & $4.6 \%$ \\
\hline
\end{tabular}

* Central Statistical Office, Government of Afghanistan

Orkand Report Note: It is not known how reliable these figures are, though the uniformity in percentages suggests some doctoring of the totals reported.

Sources: 1979 DRA Census: DRA Statistical Yearbook 1360 AH (1981-82); cited from the Orkand Report. 
Table A VI-11. GOA Estimate of Urban Population in Eastern Provinces, 1979 and 1982

\begin{tabular}{|c|c|c|c|c|c|c|}
\hline Province & 1979 Census & $\begin{array}{r}\% \text { of } \\
\text { Prov Total }\end{array}$ & $\begin{array}{r}\% \text { of } \\
\text { Natl Total }\end{array}$ & $1982 \mathrm{CSO}$ & $\begin{array}{r}\% \text { of } \\
\text { Natl Total }\end{array}$ & $\begin{array}{r}\% \text { Increase } \\
1979-82\end{array}$ \\
\hline Kabul & 919,108 & 49.3 & 46.5 & $1,036,407$ & 47.8 & $12.8 \%$ \\
\hline Kapisa & -- & -- & -- & 1,262 & 0.1 & -- \\
\hline Parvan & 25,093 & 3.0 & 1.3 & 25,117 & 1.2 & $0.1 \%$ \\
\hline Vardak & -- & -- & -- & 2,153 & 0.1 & -- \\
\hline Lowgar & -- & -- & -- & 5,259 & 0.2 & -- \\
\hline Ghazni & 30,425 & 4.7 & 1.5 & 31,985 & 1.5 & $5.1 \%$ \\
\hline Paktia & 11,415 & 2.2 & 0.6 & 12,001 & 0.5 & $5.1 \%$ \\
\hline Nangarhar & 56,384 & 7.5 & 2.9 & 60,419 & 2.8 & $7.2 \%$ \\
\hline Laghman & 3,987 & 1.3 & 0.2 & 4,191 & 0.2 & $5.1 \%$ \\
\hline Konarha & 2,089 & 0.8 & 0.1 & 2,196 & 0.1 & $5.1 \%$ \\
\hline Bamian & 7,355 & 2.7 & 0.4 & 7,732 & 0.4 & $5.1 \%$ \\
\hline Totals & $1,055,856$ & & 53.5 & $1,188,722$ & 54.9 & $12.6 \%$ \\
\hline
\end{tabular}

* Central Statistical Office, Government of Afghanistan

Orkand Report Note: It is not known how reliable these figures are, though the uniformity in percentages suggests some doctoring of the totals reported.

Sources: 1979 DRA Census: DRA Statistical Yearbook 1360 AH (1981-82); cited from the Orkand Report. 
Table A VI-12. GOA Estimate of Total Population in Northern Districts, 1979 and 1982

\begin{tabular}{|c|c|c|c|c|c|c|}
\hline Province & Rank & $\begin{array}{c}1979 \\
\text { Census }\end{array}$ & Nat'l Total $\%$ of & $1982 \mathrm{CSO}^{*}$ & $\begin{array}{c}\% \text { of } \\
\text { Nat'l Total }\end{array}$ & $\begin{array}{r}\% \text { Increase } \\
1979-82\end{array}$ \\
\hline \multicolumn{2}{|c|}{ Badakhshan } & 497,758 & 3.8 & 520,620 & 3.8 & $4.6 \%$ \\
\hline \multicolumn{2}{|c|}{ Takhar } & 519,752 & 4.0 & 543,818 & 4.0 & $4.6 \%$ \\
\hline & 493,882 & 3.8 & 516,921 & 3.8 & $4.7 \%$ \\
\hline \multicolumn{2}{|c|}{$\begin{array}{l}\text { Baghlan } \\
\text { Konduz }\end{array}$} & 555,437 & 4.3 & 582,600 & 4.2 & $4.9 \%$ \\
\hline \multicolumn{2}{|c|}{ Samangan } & 272,584 & 2.1 & 273,864 & 2.0 & $0.5 \%$ \\
\hline \multicolumn{2}{|l|}{ Balkh } & 569,255 & 4.4 & 609,590 & 4.4 & $7.1 \%$ \\
\hline \multicolumn{2}{|l|}{ Jowzjan } & 588,609 & 4.5 & 615,877 & 4.5 & $4.6 \%$ \\
\hline \multicolumn{2}{|l|}{ Faryab } & 582,705 & 4.5 & 609,703 & 4.4 & $4.6 \%$ \\
\hline \multirow[t]{2}{*}{ Badghisat } & & 233,613 & 1.8 & 244,346 & 1.8 & $4.6 \%$ \\
\hline & Total & $4,313,595$ & 33.2 & $4,517,339$ & 32.9 & $40.2 \%$ \\
\hline
\end{tabular}

* Central Statistical Office, Government of Afghanistan

Orkand Report Note: It is not known how reliable these figures are, though the uniformity in percentages suggests some doctoring of the total reported.

Source: 1979 DRA Census: DRA Statistical Yearbook 1360 AH (1981-82); cited from the Orkand Report. 
$1972 / 73$

$1973 / 74$

$1974 / 75$

$1975 / 76$

$1976 / 77$

\section{Activity rate $(\%)$}

of settled population (age $8+$ years)

\section{Economically active}

Total

40.6

Male

67.3

Female

Unemployed

Total

1.4

Male

Female

Population of working age (15-64 years)(millions)

$\begin{array}{ll}\text { Total } & 6.57 \\ \text { Settled } & 5.87 \\ \text { Male } & 3.09 \\ \text { Female } & 2.78\end{array}$

$\begin{array}{lll}6.57 & 6.72 & 6.86 \\ 5.87 & 6.00 & 6.13 \\ 3.09 & 3.16 & 3.23 \\ 2.78 & 2.84 & 2.90\end{array}$

6.86

6.13

7.01

6.26

3.30

2.96

Economically active population $(8+$ years)(millions)

Settled population
Total
Male
Female

[a] Adjusted ADS figures, reflects sex-ratio of 108 males/100 females.

Source: Afghan Demographic Survey and World Bank Mission estimates; cited from "Afghanistan: The Journey to Economic Development", Vol. II, March 1978, World Bank. 
Table A VI-14. World Bank Estimates of Labor Force

Composition Over Eight Years of Age, 1975/76

(in thousands, unless otherwise indicated)

\begin{tabular}{|c|c|c|c|c|c|c|}
\hline Sector & \multicolumn{3}{|c|}{ Settled Population only } & Nomads & $\begin{array}{c}\text { Total } \\
\text { Labor } \\
\text { Force }\end{array}$ & $\begin{array}{r}\text { As percent } \\
\text { of total } \\
\text { labor force }\end{array}$ \\
\hline Handicrafts & 115.6 & 237.5 & 353.1 & 490.5 & 843.6 & $17.9 \%$ \\
\hline Construction & 43.4 & 1.3 & 44.7 & & 44.7 & $0.9 \%$ \\
\hline Commerce & 201.5 & 1.3 & 202.8 & 54.5 & 257.3 & $5.5 \%$ \\
\hline Transport \& Communications & 56.5 & 0.1 & 56.6 & & 56.6 & $1.2 \%$ \\
\hline Unknown & 45.3 & 41.9 & 87.2 & & 87.2 & $1.9 \%$ \\
\hline Unemployed & 113.2 & 14.3 & 127.5 & & 127.5 & $2.7 \%$ \\
\hline Total & 3180.0 & 440.0 & 3620.0 & 1090.0 & 4710.0 & $100.0 \%$ \\
\hline
\end{tabular}

Source: Total labor force - Afghan Demographic Survey and World Bank Mission estimates.

Percent distribution of male and female labor force for 1972/73 - ADS, National Demographic and

Family Guidance Survey of the Settled Population of Afghanistan, 1975; cited from "Afghanistan:

The Journey to Economic Development", Vol. II, March 1978, World Bank. 
Table A VI-15. Labor Force in Afghanistan, 1969

\begin{tabular}{lrr}
\hline Occupation & Number & Percent \\
\hline Agriculture & $2,942,000$ & $79.09 \%$ \\
Misc. activities, rural areas & 250,000 & $6.72 \%$ \\
Industry, handicrafts & 231,000 & $6.21 \%$ \\
Trade & 106,000 & $2.85 \%$ \\
Construction, mining & 83,000 & $2.23 \%$ \\
Civil service & 60,000 & $1.61 \%$ \\
Transportation, communications & 30,000 & $0.81 \%$ \\
Education & 12,000 & $0.32 \%$ \\
Health & 6,000 & $0.16 \%$ \\
\multicolumn{1}{c}{$\quad$ Total } & $--1,720,000$ & $---1 .-$ \\
& & $100.00 \%$
\end{tabular}

Source: L. Dupree, "Population nevisw 1970: Afghanistan." (Washington: American Staff University Field, 1971.); citod from the Orkand Report. 
Table A VI-16. Pre-war Labor Force of

Major Industrial Enterprises, 1976/77

\begin{tabular}{llr}
\hline Name of Enterprise/Type of Production & Province & Number Employed \\
\hline Afghan Textile Company & Baghlan & 8,680 \\
Baghlan Beet Sugar Factory & Balkh & 539 \\
Balkh Ginning and Oil Extraction Mill & Balkh & 1,089 \\
Balkh Textile Factory & Balkh & 800 \\
Mazar-e Sharif Fertilizer Factory & Ghori & 4,499 \\
Cement Factory & Helmand & 678 \\
Helmand Cotton \& Vegetable Oil & Kabul & 443 \\
Afghan Mobil Carpentry & Kabul & 584 \\
Ahu Leather \& Shoe Factory & Kabul & 488 \\
Bagrami Textile Factory & Kabul & 3,130 \\
Hoechst/pharmaceuticals & Kabul & 315 \\
Jangalak/engineering Workshops & Kabul & 1,088 \\
Kabul Silo/flour and bread & Kabul & 1,307 \\
Kabul Textile & Kabul & 329 \\
Nawrozi Socks & Kabul & 69 \\
Prefabricated Concrete Plant & Kabul & 2,200 \\
Watran Plastics/plastic shoes & Kabul & 175 \\
Woolen Industries, Puli Chakri & Konduz & 723 \\
Spinzar Cotton Company & & 2,240
\end{tabular}

Source: GOA Central Statistical Office; cited from "Afghanistan: The Journey to Economic Development", Vol. II, March 1978, World Bank. 
Table A VI-17. Changes in Farm Size and Labor Force in Northern Provinces, Pre-war and 1983/84

\begin{tabular}{lrrrrr}
\hline & \multicolumn{2}{c}{ Farm Size (ha) } & \multicolumn{2}{c}{ No. of Laborers per Farm } \\
Province & Pre-War & $1983 / 84$ & $\%$ Change & Pre-War & $1983 / 84$ \\
\hline Badakhshan & 118 & 146 & $23.7 \%$ & 3.0 & 1.0 \\
Badghisat & 108 & 156 & $44.4 \%$ & 4.0 & 2.0 \\
Baghlan & 166 & 250 & $50.6 \%$ & 4.2 & 2.1 \\
Balkh & 158 & 158 & $0.0 \%$ & 5.1 & 2.0 \\
Faryab & 484 & 484 & $0.0 \%$ & 7.0 & 4.1 \\
Jowzjan & 138 & 216 & $56.5 \%$ & 4.0 & 2.0 \\
Konduz & 260 & 386 & $48.5 \%$ & 6.0 & 3.1 \\
Samangan & 236 & 338 & $43.2 \%$ & 7.0 & 3.2 \\
Takhar & 254 & 366 & $44.1 \%$ & 7.0 & 2.6 \\
& & & & & \\
\hline
\end{tabular}

Source: "Study on Agriculture in Afghanistan (1984)," by

Mohammad Ebrahim Rashid, Appendix Table Il; cited from the Orkand

Report. 
Table A VI-18. Socio-political Stratification in Afghanistan in 1973

\begin{tabular}{|c|c|c|c|}
\hline Class & Number & Members & Ethnicity \\
\hline Elite & 3,000 & $\begin{array}{l}\text { Top govt. officials; } \\
\text { military officers; } \\
\text { religious leaders; } \\
\text { wealthy businessmen; } \\
\text { local tribal and } \\
\text { ethnic leaders }\end{array}$ & $\begin{array}{l}\text { Mainly Pushtun and } \\
\text { Sunni; more minority } \\
\text { groups represented } \\
\text { after July } 1973 \text { coup }\end{array}$ \\
\hline Intelligentsia & 10,000 & $\begin{array}{l}\text { High govt. officials; } \\
\text { professionals; } \\
\text { teachers; students; } \\
\text { literati and artists; } \\
\text { mid-rank military and } \\
\text { police officers }\end{array}$ & $\begin{array}{l}\text { Mainly Pushtun, Tajik; } \\
\text { but increasing number } \\
\text { of minority group } \\
\text { individuals }\end{array}$ \\
\hline Urban Middle Class & 1 million & $\begin{array}{l}\text { Lower civil servants; } \\
\text { shopkeepers; artisans; } \\
\text { mid-range religious } \\
\text { leaders; lower level } \\
\text { military and police } \\
\text { officers }\end{array}$ & $\begin{array}{l}\text { Mainly minority groups } \\
\text { but Pushtun growing }\end{array}$ \\
\hline Lower Urban Classes & 50,000 & $\begin{array}{l}\text { Factory and } \\
\text { semi-skilled workers; } \\
\text { servants to foreigners } \\
\text { and upper class; lower } \\
\text { ranks in military and } \\
\text { police }\end{array}$ & $\begin{array}{l}\text { Mainly minority groups } \\
\text { but Pushtun growing }\end{array}$ \\
\hline Sedentary Farmers & 9.5 million & Peasant-farmers & All groups \\
\hline Non-sedentary Peoples & 3.5 million & $\begin{array}{l}\text { Semi-sedentary, semi- } \\
\text { nomadic, nomadic }\end{array}$ & $\begin{array}{l}\text { Mainly Pushtun, } \\
\text { Baluch, Turkoman; all } \\
\text { groups have some. }\end{array}$ \\
\hline
\end{tabular}

Source: L.W. Adamec, ed. "The Historical and Political Gazetteer of Afghanistan:

Marzar-i-Sharif and North-Central Afghanistan" (Graz, Austria: Akademische Druck, 1979), Vol. 4, p. 14; cited from the Orkand Report. 
Table A VI-19. Civil Administration Divisions, 1982

\begin{tabular}{|c|c|c|c|c|c|c|}
\hline Province & $\begin{array}{l}\text { Area } \\
\text { (Sq. Km.) }\end{array}$ & $\begin{array}{l}\text { Number of } \\
\text { Cities }\end{array}$ & & $\begin{array}{l}\text { er of } \\
\text { s }\end{array}$ & $\begin{array}{l}\text { Number of } \\
\text { sub-villages }\end{array}$ & $\begin{array}{r}\text { Total } \\
\text { Villages }\end{array}$ \\
\hline Badakhshan & 47,403 & & 2 & 1,352 & 244 & 1,596 \\
\hline Takhar & 12,376 & & 6 & 848 & 166 & 1,014 \\
\hline Baghlan & 17,109 & & 4 & 796 & 487 & 1,283 \\
\hline Konduz & 7,827 & & 5 & 504 & 139 & 643 \\
\hline Samangan & 15,465 & & 2 & 565 & 121 & 686 \\
\hline Balkh & 12,593 & & 4 & 806 & 141 & 947 \\
\hline Jowzjan & 25,553 & & 5 & 624 & 248 & 872 \\
\hline Faryab & 22,279 & & 4 & 762 & 164 & 926 \\
\hline Badghisat & 21,858 & & 1 & 764 & 63 & 827 \\
\hline North & 182,463 & & 33 & 7,021 & 1,773 & 8,794 \\
\hline Country & 652,225 & & 64 & 31,270 & 4,094 & 35,364 \\
\hline
\end{tabular}

Note: North is $30 \%$ of total land area, $51.7 \%$ of major cities, $22.5 \%$ of villages, $43.3 \%$ of sub-villages, $24.9 \%$ of all types of villages.

Source: "Economic and Social Indicators", March 1979-82, DRA Central Statistical Office, Part IV, no pagination; cited from the Orkand Report. 
Table A VI-20. Enrollment Growth in Education

\begin{tabular}{|c|c|c|c|c|c|c|c|c|c|c|c|}
\hline & $1964 / 65$ & $1965 / 66$ & 1966/67 & $1967 / 68$ & 1968/69 & $1969 / 70$ & 1970/71 & $1971 / 72$ & $1972 / 73$ & $1973 / 74$ & $1974 / 75$ \\
\hline \multicolumn{12}{|l|}{ Primary (grades $1-6$ ) } \\
\hline Male & 262,531 & 307,259 & 339,045 & 380,527 & 411,855 & 433,635 & 464,542 & 494,199 & 520,603 & 532,079 & 559,698 \\
\hline Female & 46,136 & 50,020 & 55,424 & 59,842 & 63,520 & 70,983 & 75,827 & 80,075 & 84,963 & 89,358 & 94,511 \\
\hline Total & 308,667 & 357,279 & 394,469 & 440,369 & 475,375 & 504,618 & 540,369 & 574,274 & 605,566 & 621,437 & 654,209 \\
\hline \multicolumn{12}{|c|}{ Middle Secondary (grades 7-9) } \\
\hline Male & 17,675 & 23,041 & 30,144 & 36,910 & 46,988 & 57,062 & 70,947 & 80,360 & 90,412 & 95,751 & 97,916 \\
\hline Female & 4,149 & 4,866 & 6,156 & 7,233 & 8,541 & 9,518 & 10,752 & 11,402 & 11,642 & 13,044 & 13,773 \\
\hline Total & 21,824 & 27,907 & 36,300 & 44,143 & 55,529 & 66,580 & 81,699 & 91,762 & 102,054 & 108,795 & 111,689 \\
\hline \multicolumn{12}{|c|}{ Upper Secondary General (grades 10-12) } \\
\hline Male & 3,458 & 4,725 & 6,018 & 8,107 & 10,724 & 13,952 & 21,926 & 30,751 & 39,759 & 45,619 & 49,686 \\
\hline Female & 1,070 & 1,354 & 1,696 & 2,147 & 2,353 & 2,997 & 3,984 & 4,642 & 5,175 & 6,044 & 6,022 \\
\hline Total & 4,528 & 6,079 & 7,714 & 10,254 & 13,077 & 16,949 & 25,910 & 35,393 & 44,934 & 51,663 & 55,708 \\
\hline \multicolumn{12}{|c|}{ Secondary Vocational (grades $7-12$ ) } \\
\hline Male & & & 4,164 & 4,043 & 4,466 & 5,149 & 4,468 & 4,052 & 3,904 & 4,178 & 3,907 \\
\hline Female & & & 370 & 443 & 452 & 476 & 486 & 475 & 506 & 551 & 580 \\
\hline Total & & & 4,534 & 4,486 & 4,918 & 5,625 & 4,954 & 4,527 & 4,410 & 4,729 & 4,487 \\
\hline \multicolumn{12}{|c|}{ Teacher Training (grades 10-17) } \\
\hline Male & & & 2,940 & 3,944 & 4,136 & 5,678 & 4,651 & 3,879 & 2,815 & 3,935 & 2,905 \\
\hline Female & & & 82 & 110 & 35 & 279 & 289 & 600 & 495 & 1,101 & 882 \\
\hline Total & & & 3,022 & 4,054 & 4,171 & 5,957 & 4,940 & 4,479 & 3,310 & 5,036 & 3,787 \\
\hline \multicolumn{12}{|l|}{ University (grades $13-18$ ) } \\
\hline Male & & & & & & & & & & 6,867 & 6,820 \\
\hline Female & & & & & & & & & & 781 & 754 \\
\hline Total & & & & & & & 5,952 & 7,183 & 7,440 & 7,648 & 7,574 \\
\hline \multicolumn{12}{|l|}{ Islamic Schools (boys) } \\
\hline Primary (grades 1-6) & 962 & 883 & 784 & 995 & 787 & 1,366 & 998 & 1,083 & 1,030 & 1,125 & 867 \\
\hline Secondary (grades $7-12$ ) & 665 & -- & 762 & 850 & 995 & 1,366 & 1,428 & 1,670 & 2,063 & 2,261 & 2,460 \\
\hline Total & 1,627 & -- & 1,546 & 1,845 & 1,782 & 2,732 & 2,426 & 2,753 & 3,093 & 3,386 & 3,327 \\
\hline
\end{tabular}

Source: GOA Ministry of Planning, Department of Education; and Kjell Nystrom, UNESCO Resident Specialist; cited from "Afghanistan: The Journey to

Economic Development", Vol. II, March 1978, World Bank. 


\begin{tabular}{|c|c|c|c|c|c|}
\hline & $1979 / 80$ & $1980 / 81$ & $1981 / 8 ?$ & \multicolumn{2}{|l|}{$\begin{array}{l}\text { UNDP Report } \\
\text { of April } 1988\end{array}$} \\
\hline $\begin{array}{l}\text { Number of hospitals } \\
\text { Number of hospital beds }-86 / 87 \\
\text { Number of doctors }\end{array}$ & 82 & 84 : & 83 & \multicolumn{2}{|l|}{$\begin{array}{l}5,100 \\
2,400\end{array}$} \\
\hline Basic health centers \& clinics & 250 & 286. & 261 & \multicolumn{2}{|c|}{$98[a]$} \\
\hline $\begin{array}{l}\text { Primary school enrollment ( } 86 / 87) \\
\text { Percent of primary school age population }\end{array}$ & & & & \multicolumn{2}{|l|}{$\begin{array}{r}615,378 \\
18.0 \%\end{array}$} \\
\hline Secondary school enrollment & & & , & \multicolumn{2}{|l|}{85,176} \\
\hline $\begin{array}{l}\text { Vocational school enrollment } \\
\text { Percent of overall secondary level enrollment }\end{array}$ & & & & \multicolumn{2}{|l|}{$\begin{array}{r}18,147 \\
18.0 \%\end{array}$} \\
\hline $\begin{array}{l}\text { Enrollment in Technicoms } \\
\text { (Soviet supported vocational schools) }\end{array}$ & & & & \multicolumn{2}{|l|}{3,569} \\
\hline Higher education enrollment & & & & \multicolumn{2}{|l|}{17,000} \\
\hline $\begin{array}{l}\text { Higher education students matriculated in 1986/87 } \\
\text { Number and Percent Enrolled in: }\end{array}$ & & & & \multicolumn{2}{|l|}{6,300} \\
\hline Faculty of agriculture & & & & 567 & $9.0 \%$ \\
\hline Polytechnic Institute & & & & 693 & $11.0 \%$ \\
\hline Medical colleges & & & & 882 & $14.0 \%$ \\
\hline Faculty of engineering & & & & 126 & $2.0 \%$ \\
\hline Natural sciences & & & & 517 & $8.2 \%$ \\
\hline Faculty of pharmacy & & & & 214 & $3.4 \%$ \\
\hline Veterinary sciences & & & & 164 & $2.6 \%$ \\
\hline Geology & & & & 176 & $2.8 \%$ \\
\hline Social sciences & & & & 2,961 & $47.0 \%$ \\
\hline $\begin{array}{l}\text { Number of graduates from colleges \& universities }(86 / 87) \\
\text { Number and Percent from: }\end{array}$ & & & & \multicolumn{2}{|l|}{2,252} \\
\hline Faculty of medecine & & & & 473 & $21.0 \%$ \\
\hline Polytechnic institute & & & & 203 & $9.0 \%$ \\
\hline Faculty of agriculture & & & & 158 & $7.0 \%$ \\
\hline Faculty of engineering & & & & 45 & $2.0 \%$ \\
\hline Faculty of natural sciences & & & & 135 & $6.0 \%$ \\
\hline Faculty of pharmacy & & & & 61 & $2.7 \%$ \\
\hline Faculty of veterinary sciences & & & & 41 & $1.8 \%$ \\
\hline Faculty of geology & & & & 32 & $1.4 \%$ \\
\hline Faculties of social sciences & & & & 1,106 & $49.1 \%$ \\
\hline $\begin{array}{l}\text { Life expectancy } \\
\text { Infant mortality }\end{array}$ & & & & $\begin{array}{c}41 \\
182-260 / 1000\end{array}$ & \\
\hline
\end{tabular}

[a] Does not include clinics.

Source: The data for 1979/80-1981/82 are from the DRA Statistical Yearbook, 1360 (March 1981 - March 1982); other data are from the UNDP Discussion Paper on Afghanistan of April 1988. 
Agricultural Population and Land Ownership in Selected Provinces 1978-1979

\begin{tabular}{|c|c|c|c|c|c|}
\hline & $\begin{array}{l}\text { Agricultural/wc } \\
\text { Population(a) } \\
(1978-1979)\end{array}$ & $\begin{array}{c}\text { Area Cultivated } \\
\text { (hectare)(b) } \\
(1971)\end{array}$ & $\begin{array}{l}\text { Land per } \\
\text { person (c) }\end{array}$ & $\begin{array}{l}\text { Number of } \\
\text { Landowners }(b, d) \\
\qquad(1971)\end{array}$ & $\begin{array}{c}\text { Average Size } \\
\text { Holding (ha) } \\
(1971)\end{array}$ \\
\hline \multicolumn{6}{|c|}{ Selected provinces } \\
\hline Kabul & 365,794 & 65,528 & 0.18 & 35,430 & 1.85 \\
\hline Logar & 191,191 & 62,017 & 0.32 & 24,840 & 2.50 \\
\hline Nangarhar & 620,684 & 59,785 & 0.10 & 87,760 & 0.68 \\
\hline Paktia (e) & 425,331 & 66,600 & 0.16 & 60,761 & 1.09 \\
\hline Paktika & 219,456 & 46,600 & 0.21 & 31,285 & 1.48 \\
\hline Konarha & 223,231 & 25,602 & 0.11 & 25,700 & 1.00 \\
\hline Kunduz & 403,443 & 286,072 & 0.70 & 32,050 & 8.92 \\
\hline Baghlan & 377,813 & 222,616 & 0.58 & 32,540 & 6.84 \\
\hline Kandahar & 349,889 & 152,549 & 0.44 & 48,430 & 3.15 \\
\hline Helmand & 441,890 & 157,852 & 0.36 & 27,450 & 7.75 \\
\hline Herat & 514,257 & 326,471 & 0.63 & 68,150 & 4.79 \\
\hline Ghazni & 554,547 & 155,200 & 0.28 & 53,184 & 2.91 \\
\hline Subtotal & $4,687,526$ & $1,626,892$ & 0.35 & 527,580 & 3.08 \\
\hline Other provinces & $5,294,828$ & $2,250,741$ & 0.48 & 658,530 & 3.83 \\
\hline Total & $9,982,354$ & $4,147,633$ & 0.40 & $1,186,110$ & 3.50 \\
\hline
\end{tabular}

(a) Rural population was reduced by 10 percent to produce these figures. Data on rural population are presented in Macroeconomic Database Development, Nathan Associates, Appendices, January 1990, Tables A V1-5 and A V1-6.

(b) Based on the summary results of the preliminary Agricultural Census of Afghanistan, 1971. A summary of these results is presented in Afghanistan: Current Economic Situation, World Bank, 1971.

(c) Land per person is based on population figures from 1978 to 1979 and area figures from 1978.

(d) It is assumed that the land ownership pattern given by the 1971 agricultural census remained unchanged upto 1978.

(e) The preliminary agricultural census does not provide data on the cultivated area and the number of landowners in Paktika province. Figures presented were derived from previous studies on Paktia and Ghazni provinces and should be regarded as preliminary. 
VII. INFRASTRUCTURE AND NATURAL RESOURCES

1. Dist. of Proposed Capital and Technical Assistance by Sectors

2. Ongoing and New Industrial Development Projects (East)

3. Ongoing and New Energy Projects (East)

4. Ongoing and New Industrial Development Projects (North)

5. Ongoing and New Energy Projects (North)

6. Ongoing and New Transport Projects (North)

7. Land Resources and Utilization, 1977

8. Ongoing and New Irrigation Projects (East)

9. Ongoing and New Irrigation Projects (North)

10. Selected Data on Infrastructure \& Resources

0. RETURN TO MAIN MENU 
Table A VII-1. Distribution of Proposed Capital and Technical Assistance by Main Economic Sectors

(millions of U.S. dollars, unless otherwise indicated)

\begin{tabular}{|c|c|c|c|c|c|c|}
\hline \multirow[b]{2}{*}{ Sector } & \multicolumn{4}{|c|}{ Capital Assistance } & \multicolumn{2}{|c|}{ Technical Assistance } \\
\hline & Domestic & External & Total & Percent & Total & Percent \\
\hline \multicolumn{7}{|l|}{ Agriculture, Rural Developement } \\
\hline 1. Agriculture & 14.0 & 65.8 & 79.8 & $8.7 \%$ & 16.0 & $25.2 \%$ \\
\hline 2. Irrigation & 39.1 & 114.9 & 154.0 & $16.8 \%$ & 2.3 & $3.6 \%$ \\
\hline 3. Rural Development & 23.4 & 51.8 & 75.2 & $8.2 \%$ & 3.5 & $5.5 \%$ \\
\hline Industry, Power and Mining & 33.7 & 111.5 & 145.2 & $15.9 \%$ & 6.0 & $9.4 \%$ \\
\hline 1. Industry & 16.2 & 63.9 & 80.1 & $8.7 \%$ & 2.0 & $3.1 \%$ \\
\hline 2. Power & 17.5 & 47.6 & 65.1 & $7.1 \%$ & 4.0 & $6.3 \%$ \\
\hline 3. Mining & -- & -- & -- & -- & -- & -- \\
\hline Transport \& Communications & 35.1 & 106.9 & 142.0 & $15.5 \%$ & 8.4 & $13.2 \%$ \\
\hline 1. Transport & 23.0 & 63.0 & 86.0 & $9.4 \%$ & 7.0 & $11.0 \%$ \\
\hline 2. Communications & 12.1 & 43.9 & 56.0 & $6.1 \%$ & 1.4 & $2.2 \%$ \\
\hline Social Services & 39.2 & 103.1 & 142.3 & $15.5 \%$ & 18.3 & $28.8 \%$ \\
\hline 1. Education & 13.9 & 40.1 & 54.0 & $5.9 \%$ & 7.0 & $11.0 \%$ \\
\hline 2. Health & 7.5 & 22.5 & 30.0 & $3.3 \%$ & 4.2 & $6.6 \%$ \\
\hline 3. Water Supply & 17.8 & 40.5 & 58.3 & $6.4 \%$ & 4.0 & $6.3 \%$ \\
\hline 4. Planning and Statistics & -- & -- & -- & -- & 3.1 & $4.9 \%$ \\
\hline Contingencies & 44.3 & 132.9 & 177.2 & $19.4 \%$ & 9.0 & $14.2 \%$ \\
\hline Total & 228.8 & 686.9 & 915.7 & $100.0 \%$ & 63.5 & $100.0 \%$ \\
\hline
\end{tabular}

Source: UNDP Discussion Paper on Afghanistan of April 1988. 
Table A VII-2. Ongoing and New Industrial

Development Projects in Eastern Provinces

\begin{tabular}{|c|c|c|c|c|c|}
\hline Province & Project Title & $\begin{array}{l}\text { Implementation } \\
\text { Period }\end{array}$ & $\begin{array}{l}\text { Cost } \\
(\$ \mathrm{~mm})\end{array}$ & Status & Remarks \\
\hline \multirow[t]{6}{*}{ Kabul } & $\begin{array}{l}\text { Expansion of } \\
\text { industrial area. }\end{array}$ & 1978 to 1985 & 2.2 & $\begin{array}{c}\text { Under } \\
\text { construction. }\end{array}$ & Indian assisted \\
\hline & $\begin{array}{l}\text { Alcoholic drinks } \\
\text { plant. }\end{array}$ & 1980 to 1985 & 3.1 & $\begin{array}{l}\text { Feasibility study } \\
\text { phase. }\end{array}$ & $\begin{array}{l}\text { Bulgaria } \\
\text { assisted. }\end{array}$ \\
\hline & $\begin{array}{l}\text { Brick making } \\
\text { plant. }\end{array}$ & 1981 to 1986 & 6.3 & $\begin{array}{c}\text { Feasibility study } \\
\text { phase. }\end{array}$ & $\begin{array}{l}\text { Bulgaria } \\
\text { assisted. }\end{array}$ \\
\hline & Mini-Steel Plant & 1983 to 1989 & 4.0 & $\begin{array}{l}\text { Feasibility study } \\
\text { phase. }\end{array}$ & Donor sought. \\
\hline & $\begin{array}{l}\text { Leather } \\
\text { manufacturing } \\
\text { complex. }\end{array}$ & 1980 to 1985 & 19.4 & $\begin{array}{l}\text { Feasibility study } \\
\text { phase. }\end{array}$ & $\begin{array}{l}\text { Bulgaria } \\
\text { assisted. }\end{array}$ \\
\hline & $\begin{array}{l}\text { Bakery } \\
\text { reconstruction }\end{array}$ & 1981 to 1985 & 4.3 & $\begin{array}{c}\text { Under } \\
\text { construction. }\end{array}$ & $\begin{array}{c}\text { Soviet } \\
\text { Assisted. } 90 \\
\text { tons bread/da! }\end{array}$ \\
\hline
\end{tabular}

Source: Report on the Socio-Economic Situation Development Strategy Assistance Needs, Vol. I, DRA, 1983, pp.121-132; cited from the Orkand Report. 
Table A VII-3. Ongoing and New Energy

Projects in Eastern Provinces

\begin{tabular}{|c|c|c|c|c|c|}
\hline Province & Project Title & $\begin{array}{l}\text { Implementation } \\
\text { Period }\end{array}$ & $\begin{array}{l}\text { Cost } \\
(\$ \mathrm{~mm})\end{array}$ & Status & Remarks \\
\hline Nangarhar & $\begin{array}{l}110 \mathrm{KV} \\
\text { Transmission Line } \\
\text { Naghlu to } \\
\text { Jalalabad. }\end{array}$ & 1978 to 1983 & 6.6 & $\begin{array}{c}\text { Under } \\
\text { construction. }\end{array}$ & 95 km. \\
\hline Kabul & $\begin{array}{l}\text { Distribution } \\
\text { Network Phase III }\end{array}$ & 1967 to 1984 & 5.9 & $\begin{array}{c}\text { Under } \\
\text { construction. }\end{array}$ & GDR funded \\
\hline Kabul & $\begin{array}{l}220 \mathrm{KV} \\
\text { Trans-Kabul to } \\
\text { USSR }\end{array}$ & 1981 to 1989 & 26.3 & Projected. & USSR fundec \\
\hline Kabul & $\begin{array}{l}60 \text { MW Distrib } \\
\text { Network, Fourth } \\
\text { Phase }\end{array}$ & 1979 to ? & 27.0 & $\begin{array}{l}\text { Feasibility in } \\
\text { progress. }\end{array}$ & GDR assistec \\
\hline Parvan & $\begin{array}{l}\text { Extension of } \\
\text { Kajaki } \\
\text { Hydro-Power } \\
\text { Station \& } 220 \mathrm{KV} \\
\text { Transmission } \\
\text { Line, First Stage. }\end{array}$ & 1981 to 1986 & 20.0 & Feasibility. & $\begin{array}{l}\text { Asian Devt. } \\
\text { Bank assist } \\
\text { suspended. }\end{array}$ \\
\hline
\end{tabular}

Source: Report on the Socio-Economic Situation Development Strategy Assistance Needs, Vol.

I, DRA, 1983, pp.133-138; cited from the Orkand Report. 
Table A VII-4. Ongoing and New Industrial Development Projects in Northern Provinces

\begin{tabular}{|c|c|c|c|c|c|}
\hline Province & Project Title & $\begin{array}{l}\text { Implementation } \\
\text { Period }\end{array}$ & $\begin{array}{l}\text { Cost } \\
(\$ \mathrm{~mm})\end{array}$ & Status & Remarks \\
\hline Balkh & $\begin{array}{l}\text { Gas Turbine No.4 } \\
\text { and Steam Engine } \\
\text { No. } 5\end{array}$ & 1978 to 1984 & 7.9 & $\begin{array}{c}\text { Under } \\
\text { construction. }\end{array}$ & $\begin{array}{c}\text { Soviet } \\
\text { assisted. } 72 \\
\text { MW capacity. }\end{array}$ \\
\hline & Fertilizer Project & 1977 to 1988 & 154.5 & $\begin{array}{l}\text { Feasibility } \\
\text { study phase. }\end{array}$ & $\begin{array}{c}\text { Soviet } \\
\text { assisted. } \\
335,000 \text { ton } \\
\text { per yer } \\
\text { capacity. }\end{array}$ \\
\hline & $\begin{array}{l}\text { Mazar-e Sharif } \\
\text { Silos }\end{array}$ & 1976 to 1982 & 3.4 & $\begin{array}{c}\text { Under } \\
\text { construction. }\end{array}$ & $\begin{array}{l}\text { Soviet } \\
\text { assisted. }\end{array}$ \\
\hline & $\begin{array}{l}\text { Mazar-e Sharif } \\
\text { Mills }\end{array}$ & 1976 to 1983 & 1.6 & $\begin{array}{l}\text { Under } \\
\text { construction. }\end{array}$ & $\begin{array}{c}\text { Soviet } \\
\text { assisted. } 60 \\
\text { tons of } \\
\text { wheat/day } \\
\text { capacity. }\end{array}$ \\
\hline & Bakery (in Mazar) & 1976 to 1983 & 2.0 & $\begin{array}{c}\text { Under } \\
\text { construction. }\end{array}$ & $\begin{array}{l}\text { Soviet } \\
\text { assisted. } 25 \\
\text { tons of } \\
\text { bread/day } \\
\text { capacity. }\end{array}$ \\
\hline Badghisat & Gin and Press & 1977 to 1987 & 2.0 & $\begin{array}{c}\text { Partly } \\
\text { implemented. }\end{array}$ & $\begin{array}{c}\text { Needs } \\
\text { funding. } \\
11,000 \text { tons } \\
\text { per year } \\
\text { capacity. }\end{array}$ \\
\hline \multirow[t]{2}{*}{ Baghlan } & Sugar Project & 1977 to 1985 & 46.7 & $\begin{array}{c}\text { Under } \\
\text { construction. }\end{array}$ & $\begin{array}{l}\text { UAE and } \\
\text { Kuwaiti } \\
\text { assistance. } \\
27,000 \text { tons } \\
\text { per year } \\
\text { capacity. }\end{array}$ \\
\hline & Pul-e Khomri Mills & 1976 to 1983 & 2.4 & $\begin{array}{c}\text { Under } \\
\text { construction. }\end{array}$ & $\begin{array}{l}\text { Soviet } \\
\text { assisted. }\end{array}$ \\
\hline
\end{tabular}

Source: Report on the Socio-Economic Situation Development Strategy Assistance Needs, Vol. I, DRA, 1983, pp.121-132; cited from the Orkand Report. 
Table A VII-5. Ongoing and New Energy

Projects in Northern Provinces

\begin{tabular}{|c|c|c|c|c|}
\hline Province & Project Title & $\begin{array}{l}\text { Implementation } \\
\text { Period }\end{array}$ & $\begin{array}{r}\text { Cost } \\
(\$ \mathrm{~mm})\end{array}$ & Remarks \\
\hline Badakshan & $\begin{array}{l}\text { Hydro-electric } \\
\text { power station }\end{array}$ & 1976 to 1983 & 1.0 Under construction & $\begin{array}{l}.75 \mathrm{MW} \\
\text { capacity }\end{array}$ \\
\hline Samangan & $\begin{array}{l}\text { Hydro-electric } \\
\text { power station }\end{array}$ & 1976 to 1983 & 1.5 Under construction & $\begin{array}{r}.50 \mathrm{MW} \\
\text { capacity }\end{array}$ \\
\hline \multirow[t]{2}{*}{ Balkh } & $\begin{array}{l}\text { Distribution network } \\
\text { system in Mazar }\end{array}$ & 1970 to 1983 & 3.8 Under construction & $\begin{array}{l}15.5 \mathrm{MW} \\
\text { capacity }\end{array}$ \\
\hline & $\begin{array}{l}\text { Extension of } 110 \mathrm{KV} \text { sub } \\
\text { stations \& transmission } \\
\text { lines in Mazar \& Balkh }\end{array}$ & 1982 to 1985 & 0.1 Plans in progress & $\begin{array}{r}\text { Soviet } \\
\text { assisted }\end{array}$ \\
\hline \multirow[t]{2}{*}{ Konduz } & $\begin{array}{l}110 \mathrm{KV} \text { transmission } \\
\text { line to USSR }\end{array}$ & 1982 to 1989 & 3.4 Proposed & $\begin{array}{r}\text { Soviet } \\
\text { assisted }\end{array}$ \\
\hline & $\begin{array}{l}\text { Electrical distribution } \\
\text { network }\end{array}$ & 1982 to 1984 & 1.6 Proposed & $\begin{array}{r}\text { Assistance } \\
\text { needed }\end{array}$ \\
\hline
\end{tabular}

Source: Report on the Socio-Economic Situation Development Strategy Assistance Needs, Vol.

I, DRA, 1983, pp.133-138; cited from the Orkand Report. 
Table A VII-6. Ongoing and New Transport

Projects in Northern Provinces

\begin{tabular}{|c|c|c|c|c|}
\hline Province & Project Title & $\begin{array}{l}\text { Implementation } \\
\text { Period }\end{array}$ & $\begin{array}{r}\text { Cost } \\
(\$ \mathrm{~mm})\end{array}$ & Remarks \\
\hline $\begin{array}{l}\text { Baghlan, } \\
\text { Balkh, \& } \\
\text { Samangan }\end{array}$ & $\begin{array}{l}\text { Workshops for } \\
\text { trans. equipment in } \\
\text { Pol-e-Khomri Hairatan } \\
\text { and Torghundi }\end{array}$ & 1980 to 1984 & 1.7 Under construction & $\begin{array}{r}\text { Soviet } \\
\text { assisted }\end{array}$ \\
\hline $\begin{array}{l}\text { Konduz \& } \\
\text { Faryab }\end{array}$ & $\begin{array}{l}\text { Expansion \& equipping } \\
\text { of domestic airports }\end{array}$ & 1967 to 1985 & In progress & $\begin{array}{l}\text { Locally } \\
\text { funded }\end{array}$ \\
\hline Badakshan & $\begin{array}{l}\text { Reconstruction of } \\
\text { airports }\end{array}$ & 1976 to 1986 & 5.2 Under construction & $\begin{array}{r}\text { Soviet } \\
\text { assisted }\end{array}$ \\
\hline \multirow[t]{2}{*}{ Balkh } & $\begin{array}{l}\text { Construction of oil } \\
\text { stores in Mazar }\end{array}$ & 1981 to 1983 & 5.8 Under construction & $\begin{array}{r}\text { Soviet } \\
\text { assisted }\end{array}$ \\
\hline & $\begin{array}{l}\text { Construction of oil } \\
\text { tanker transport base }\end{array}$ & 1980 to 1983 & 1.3 Under construction & $\begin{array}{r}\text { Soviet } \\
\text { assisted }\end{array}$ \\
\hline Badghisat & Airport reconstruction & 1982 to 1984 & - - Under construction & $\begin{array}{l}\text { Locally } \\
\text { funded }\end{array}$ \\
\hline
\end{tabular}

Source: Report on the Socio-Economic Situation Development Strategy Assistance Needs, Vol.

I, DRA, 1983, pp.145-147; cited from the Orkand Report. 
A. Categories of Land ('OOO ha)

\begin{tabular}{lclr} 
Type of Land & Area & Land Use & Area \\
\hline Arable & - & Uncultivated & Irrigated [a] \\
$\quad$ Uncultivated & 7,910 & Rainfed [a] & 2,900 \\
Cultivated & 3,900 & Tree crops & 1,424 \\
& 4,010 & Grazing & 372 \\
Permanent Crops & & Forest & 54,700 \\
Permanent Pasture & 372 & Summer grazing & 1,900 \\
Forest & 54,700 & Winter grazing & 22,460 \\
Mountains \& Desert & 1,900 & Non-agricultural & 16,210 \\
& 381 & & 381 \\
Total Land Area & & & 65,263 \\
\hline
\end{tabular}

[a] Of the irrigated land $26 \%$ is estimated to be fallowed annually while $40 \%$ of rainfed land is fallowed.

\begin{tabular}{|c|c|}
\hline \multicolumn{2}{|l|}{ B. Sources of Irrigation Water } \\
\hline Source & Area ('000 ha) \\
\hline Canals & 2,210 \\
\hline Springs & 190 \\
\hline Karezes & 170 \\
\hline Wells & 16 \\
\hline & 2,586 \\
\hline
\end{tabular}

C. Average Crop Area ('000 ha), 1971/72-76/77

$\begin{array}{lrrr} & \text { Irrigated } & \text { Rainfed } & \text { Total } \\ \text { Wheat } & 1,314 & 1,096 & 2,410 \\ \text { Maize } & 479 & 0 & 479 \\ \text { Rice } & 208 & 0 & 208 \\ \text { Barley } & 110 & 211 & 321 \\ \text { Seed cotton } & 93 & 0 & 93 \\ \text { Sugar beet/cane } & 7 & 0 & 7 \\ \text { Oil seeds } & 19 & 36 & 55 \\ \text { Vegetables } & 100 & 0 & 100 \\ \text { Fruits } & 138 & 0 & 138 \\ \text { Alfalfa, clover } & 60 & 0 & 60 \\ \text { Other } & 75 & 0 & 75 \\ & ----1,343 & --1,946\end{array}$


Table A VII-8. Ongoing and New Irrigation

Projects in Eastern Provinces

$(1983-1989)$

\begin{tabular}{|c|c|c|c|c|c|}
\hline Province & Project Title & $\begin{array}{l}\text { Implementation } \\
\text { Period }\end{array}$ & $\begin{array}{r}\text { Cost } \\
(\$ \mathrm{~mm})\end{array}$ & Status & Remarks \\
\hline Parvan & Parvan Irrigation & 1977 to 1984 & 16.3 & Mostly completed. & $\begin{array}{l}\text { PRC assistance } \\
\text { suspended. }\end{array}$ \\
\hline Ghazni & Sardeh Dam & 1961 to 1986 & 12.4 & $\begin{array}{l}\text { Dam \& canal } \\
\text { completed. Farm } \\
\text { development in } \\
\text { progress. }\end{array}$ & USSR assisted. \\
\hline Kabul & $\begin{array}{l}\text { Rehab of canals \& } \\
\text { water storage. }\end{array}$ & 1983 to 1992 & 6.5 & Under study. & Donor sought. \\
\hline Nangarhar & Kama Irrigation & 1984 to 1992 & 20.0 & Under study. & $\begin{array}{l}\text { ADB funding } \\
\text { sought. }\end{array}$ \\
\hline Parvan & $\begin{array}{l}\text { Panjsher Valley } \\
\text { rehabilitation of } \\
\text { dams, irrigation } \\
\text { systems. }\end{array}$ & 1985 to 1994 & 80.0 & Under study. & Lack of funds. \\
\hline
\end{tabular}

Source: Report on the Socio-Economic Situation Development Strategy Assistance Needs, Vol. I, DRA, 1983, pp.113-120; cited from the Orkand Report. 
Table A VII-9. Ongoing and New Irrigation Projects in Northern Provinces (1983-1989)

\begin{tabular}{|c|c|c|c|c|c|}
\hline Province & Project Title & $\begin{array}{l}\text { Implementation } \\
\text { Period }\end{array}$ & $\begin{array}{r}\text { Cost } \\
(\$ \mathrm{~mm})\end{array}$ & Status & Remarks \\
\hline \multirow[t]{3}{*}{ Baghlan } & $\begin{array}{l}\text { Gawarghan } \\
\text { Chardaran } \\
\text { Irrigation }\end{array}$ & 1972 to 1985 & 15.95 & $\begin{array}{l}\text { Under } \\
\text { construction. }\end{array}$ & $\begin{array}{l}\text { Part of } \\
\text { project in } \\
\text { use. }\end{array}$ \\
\hline & $\begin{array}{l}\text { Kilaghai } \\
\text { Irrigation }\end{array}$ & 1985 to 1993 & 96.00 & $\begin{array}{l}\text { Detailed } \\
\text { design } \\
\text { complete }\end{array}$ & $\begin{array}{c}\text { Soviet } \\
\text { assisted. }\end{array}$ \\
\hline & $\begin{array}{l}\text { Aliabad Pump } \\
\text { Irrigation }\end{array}$ & 1983 to 1985 & 5.80 & $\begin{array}{l}\text { Design } \\
\text { complete }\end{array}$ & \\
\hline \multirow[t]{2}{*}{ Takhar } & $\begin{array}{l}\text { Kowkcheh Sufla } \\
\text { Irrigation }\end{array}$ & 1976 to 1988 & 46.06 & $\begin{array}{c}\text { Feasibility } \\
\text { study complete }\end{array}$ & $\begin{array}{l}\text { Soviet } \\
\text { assisted. }\end{array}$ \\
\hline & Kowkcheh Sufla & 1983 to 1989 & 46.00 & $\begin{array}{l}\text { Ready to begin } \\
\text { construction }\end{array}$ & $\begin{array}{l}\text { Soviet } \\
\text { assisted. }\end{array}$ \\
\hline \multirow[t]{2}{*}{ Konduz } & $\begin{array}{l}\text { Khanabad } \\
\text { Irrigation } \\
\text { Phase I }\end{array}$ & 1976 to 1985 & 14.70 & $90 \%$ completed & $\begin{array}{l}\text { Barrage and } \\
\text { most lined } \\
\text { canals } \\
\text { completed. }\end{array}$ \\
\hline & $\begin{array}{l}\text { Khanabad } \\
\text { Irrigation } \\
\text { Phase II }\end{array}$ & 1983 to 1989 & 15.42 & $\begin{array}{c}\text { Feasibility } \\
\text { study accepted by } \\
\text { World Bank }\end{array}$ & $\begin{array}{l}\text { Requires } \\
\text { World Bank } \\
\text { resumption } \\
\text { of funding. }\end{array}$ \\
\hline \multirow[t]{2}{*}{ Jowzjan } & $\begin{array}{l}\text { Koshk Morghab } \\
\text { River Protection }\end{array}$ & 1981 to 1984 & 33.00 & $\begin{array}{l}\text { Design complete } \\
\text { ready to build }\end{array}$ & $\begin{array}{l}\text { Soviet } \\
\text { assisted. }\end{array}$ \\
\hline & $\begin{array}{l}\text { Qeysar Nurghab } \\
\text { Irrigation }\end{array}$ & 1983 to 1985 & 2.15 & $\begin{array}{l}\text { Feasibility } \\
\text { study needed }\end{array}$ & Needs funding \\
\hline \multirow[t]{3}{*}{ Balkh } & $\begin{array}{l}\text { Chashma Shafa } \\
\text { Irrigation }\end{array}$ & 1985 to 1988 & 18.00 & $\begin{array}{l}\text { Feasibility } \\
\text { established }\end{array}$ & $\begin{array}{c}\text { Soviet } \\
\text { assisted. }\end{array}$ \\
\hline & $\begin{array}{l}\text { Khoshtepa } \\
\text { Irrigation }\end{array}$ & 1984 to 1993 & 69.00 & $\begin{array}{l}\text { Design complete } \\
\text { ready to build }\end{array}$ & $\begin{array}{l}\text { Soviet } \\
\text { assisted. }\end{array}$ \\
\hline & $\begin{array}{l}\text { Rehabilitation of } \\
\text { Shahi Canal }\end{array}$ & 1983 to 1985 & 10.00 & $\begin{array}{c}\text { Feasibility } \\
\text { prepared by Sanyo } \\
\text { of Japan }\end{array}$ & Needs funding \\
\hline Faryab & $\begin{array}{l}\text { Feasibility Study } \\
\text { of Shabaktu Dam }\end{array}$ & 1983 to 1984 & 1.50 & $\begin{array}{c}\text { Preliminary } \\
\text { study complete }\end{array}$ & Needs funding \\
\hline $\begin{array}{l}\text { Northern } \\
\text { Provinces }\end{array}$ & $\begin{array}{l}\text { Rehabilitation of } \\
\text { Canals in } \\
\text { Northern Region }\end{array}$ & 1979 to 1986 & 5.38 & $\begin{array}{l}\text { Studies and } \\
\text { surveys completed }\end{array}$ & $\begin{array}{l}\text { Soviet } \\
\text { assisted. }\end{array}$ \\
\hline
\end{tabular}

Source: Report on the Socio-Economic Situation Development Strategy Assistance Needs, Vol. I, DRA, 183, pp.145-147; cited from the Orkand Report. 
Table A VII-10. Select Data on Afghan Infrastructure and Resources

\begin{tabular}{|c|c|c|c|c|}
\hline & $1979 / 80$ & $1980 / 81$ & $1981 / 82$ & $\begin{array}{l}\text { UNDP Report } \\
\text { of April } 1988\end{array}$ \\
\hline $\begin{array}{l}\text { Total length of roads ( } 00 \text { of } \mathrm{Kms} \text {.) } \\
\text { Of which: }\end{array}$ & 18,790 & 18,852 & 18,974 & 170 \\
\hline $\begin{array}{l}\text { Asphalted roads } \\
\text { (Percent of total) }\end{array}$ & $\begin{array}{r}2,784 \\
14.8 \%\end{array}$ & $\begin{array}{r}2,846 \\
15.1 \%\end{array}$ & $\begin{array}{r}2,858 \\
15.1 \%\end{array}$ & \\
\hline $\begin{array}{l}\text { Gravel roads } \\
\text { (Percent of total) }\end{array}$ & & & & $\begin{array}{r}43 \\
25.3 \%\end{array}$ \\
\hline $\begin{array}{l}\text { Earth roads } \\
\text { (Percent of total) }\end{array}$ & & & & $\begin{array}{r}100 \\
58.8 \%\end{array}$ \\
\hline $\begin{array}{l}\text { Paved roads } \\
\text { (Percent of total) }\end{array}$ & $\begin{array}{r}5,090 \\
27.1 \%\end{array}$ & $\begin{array}{r}5,120 \\
27.2 \%\end{array}$ & $\begin{array}{r}5,175 \\
27.3 \%\end{array}$ & $\begin{array}{r}27 \\
15.9 \%\end{array}$ \\
\hline $\begin{array}{l}\text { Iron ore deposits } \\
\text { (billions of tons) }\end{array}$ & & & & 1.7 \\
\hline $\begin{array}{l}\text { Copper } \\
\text { (millions of tons) }\end{array}$ & & & & 5 \\
\hline $\begin{array}{l}\text { Coal } \\
\text { (millions of tons) }\end{array}$ & & & & 400 \\
\hline $\begin{array}{l}\text { Petroleum reserves } \\
\text { (millions of tons) }\end{array}$ & & & & 20 \\
\hline $\begin{array}{l}\text { Total installed electrical capacity (MW) } \\
\text { Of which: } \\
\text { Hydro-electric } \\
\text { (percent of total) }\end{array}$ & & & & $\begin{array}{r}408 \\
256 \\
62.7 \%\end{array}$ \\
\hline $\begin{array}{l}\text { Gas power } \\
\text { (percent of total) }\end{array}$ & & $\therefore$ & & $\begin{array}{r}48 \\
11.8 \%\end{array}$ \\
\hline $\begin{array}{l}\text { Diesel generated } \\
\text { (percent of total) }\end{array}$ & & & & $\begin{array}{r}104 \\
25.5 \%\end{array}$ \\
\hline
\end{tabular}




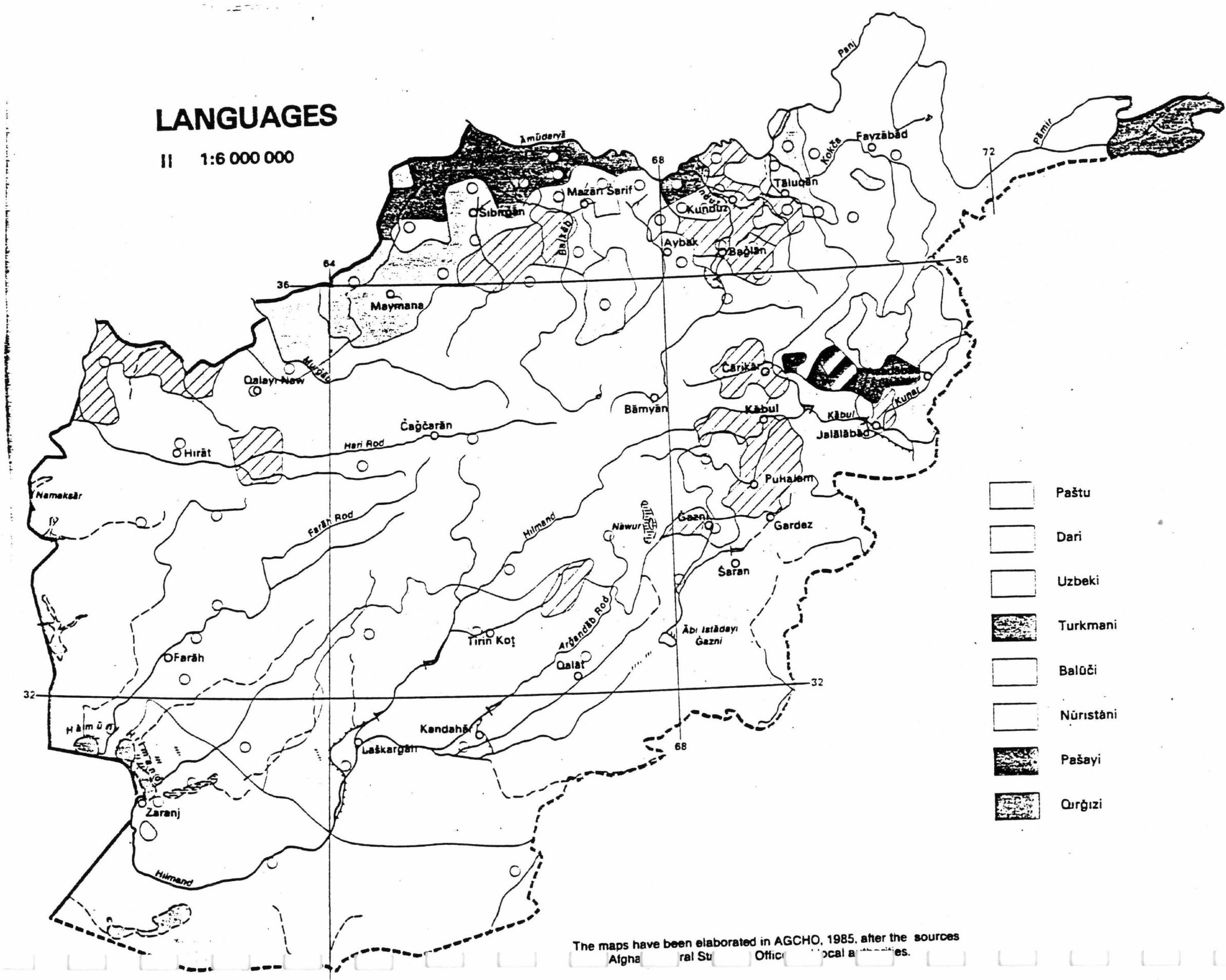




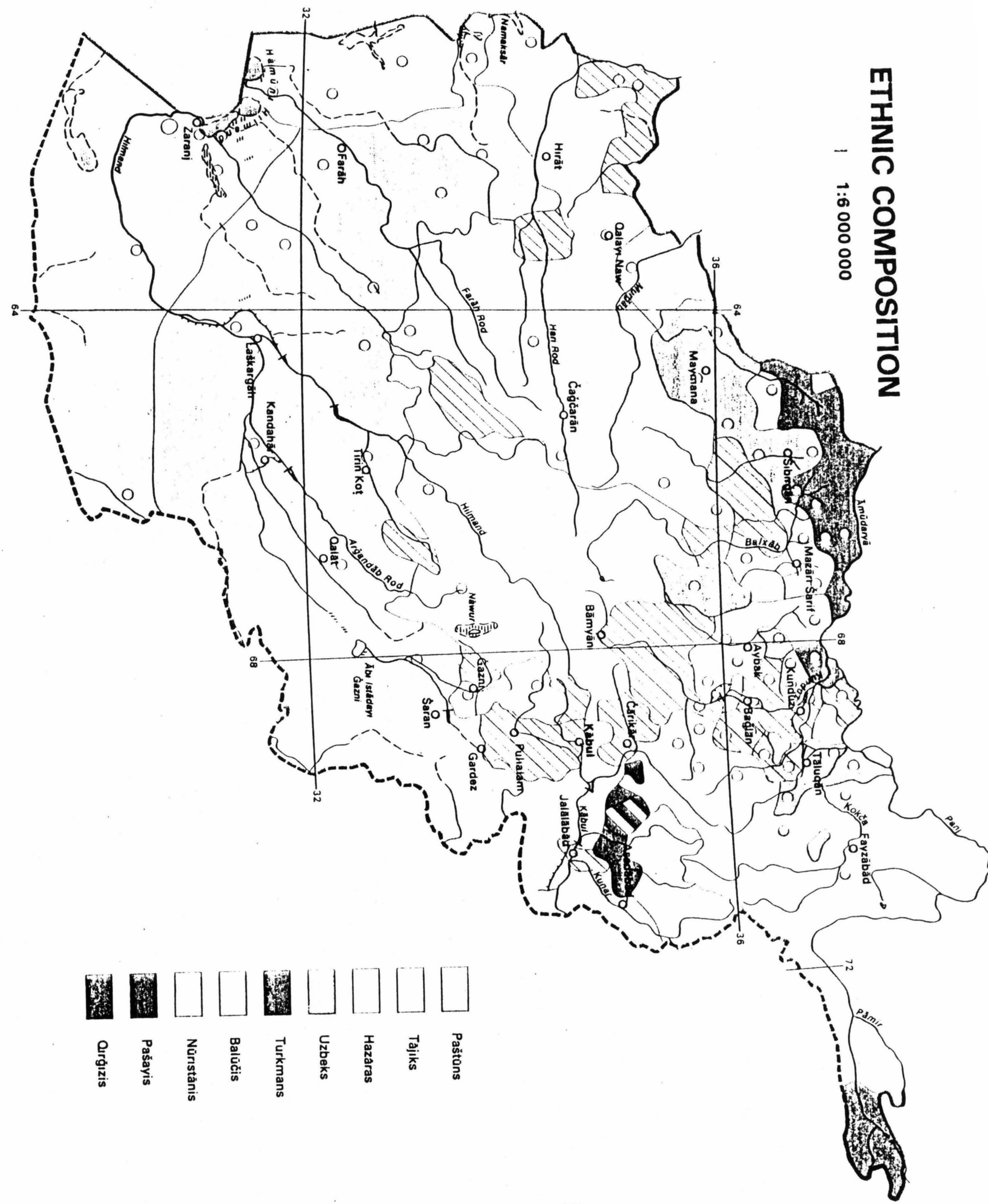




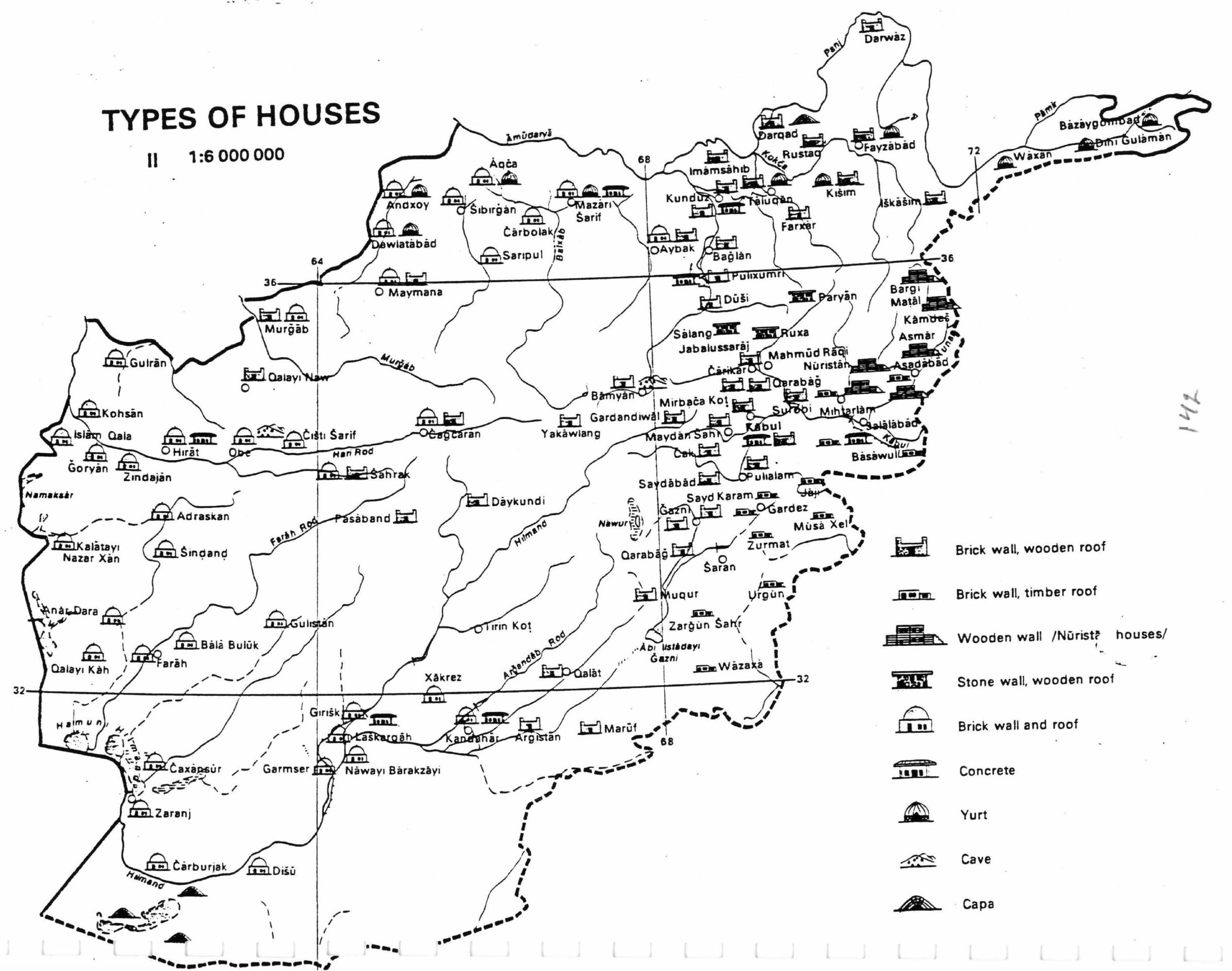




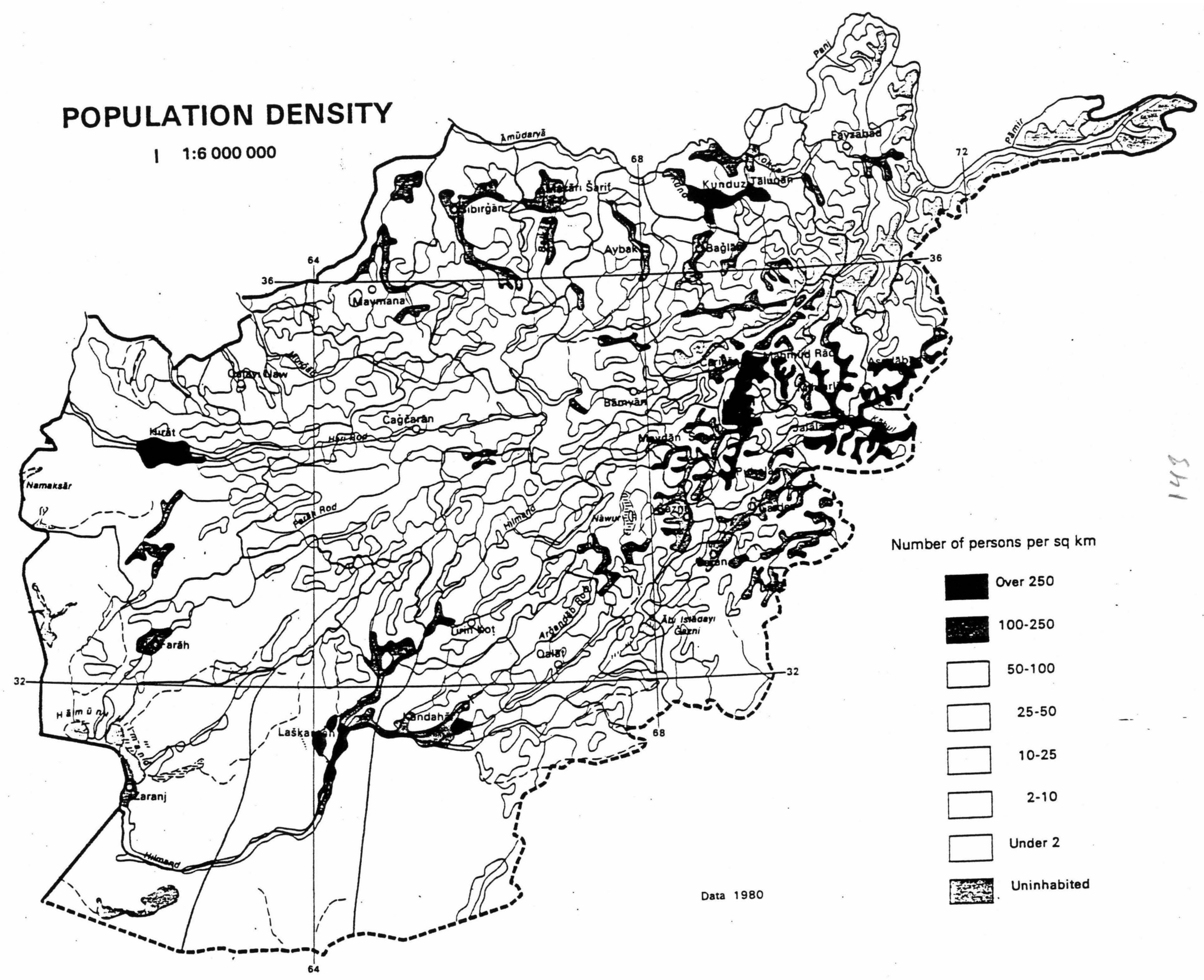




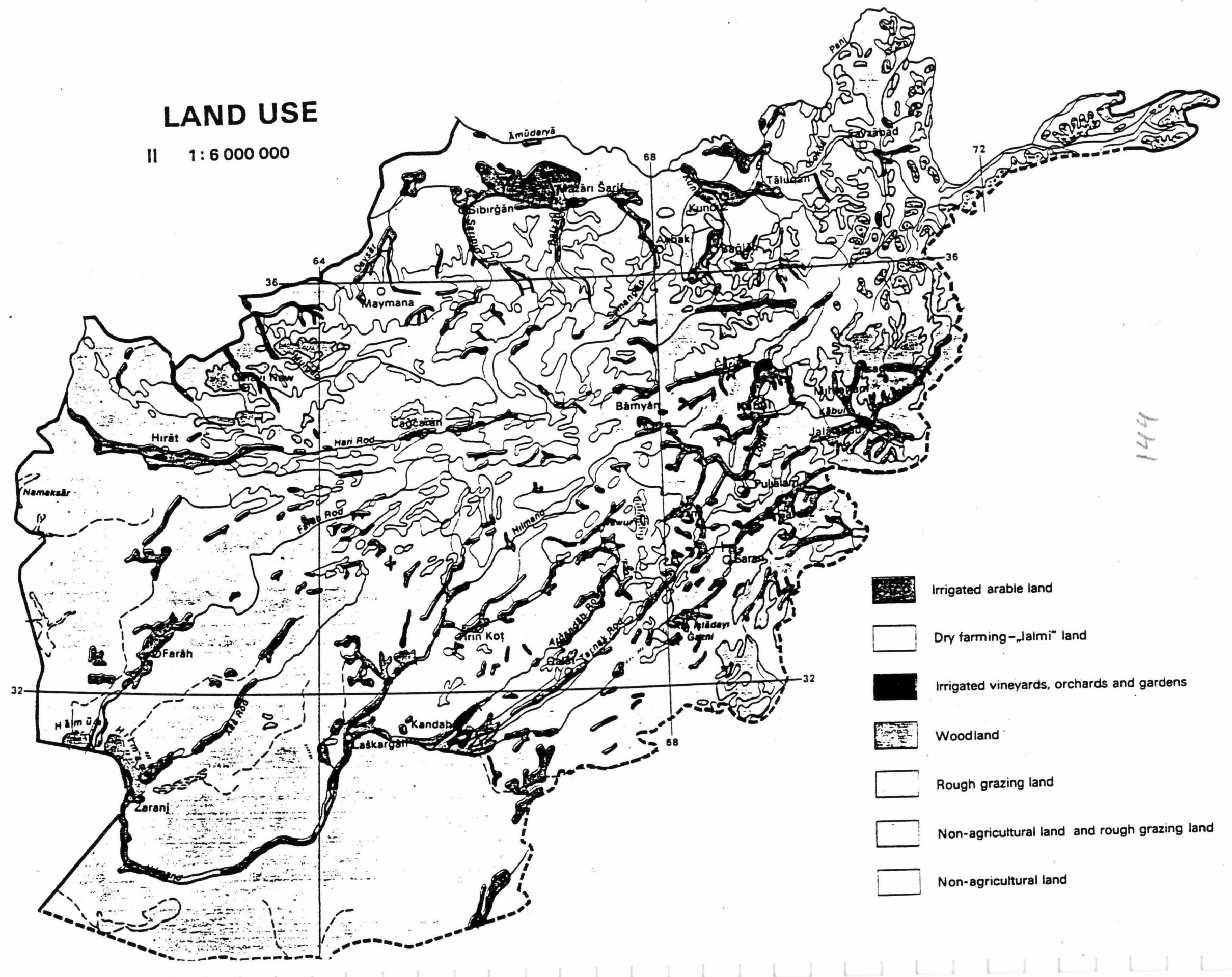




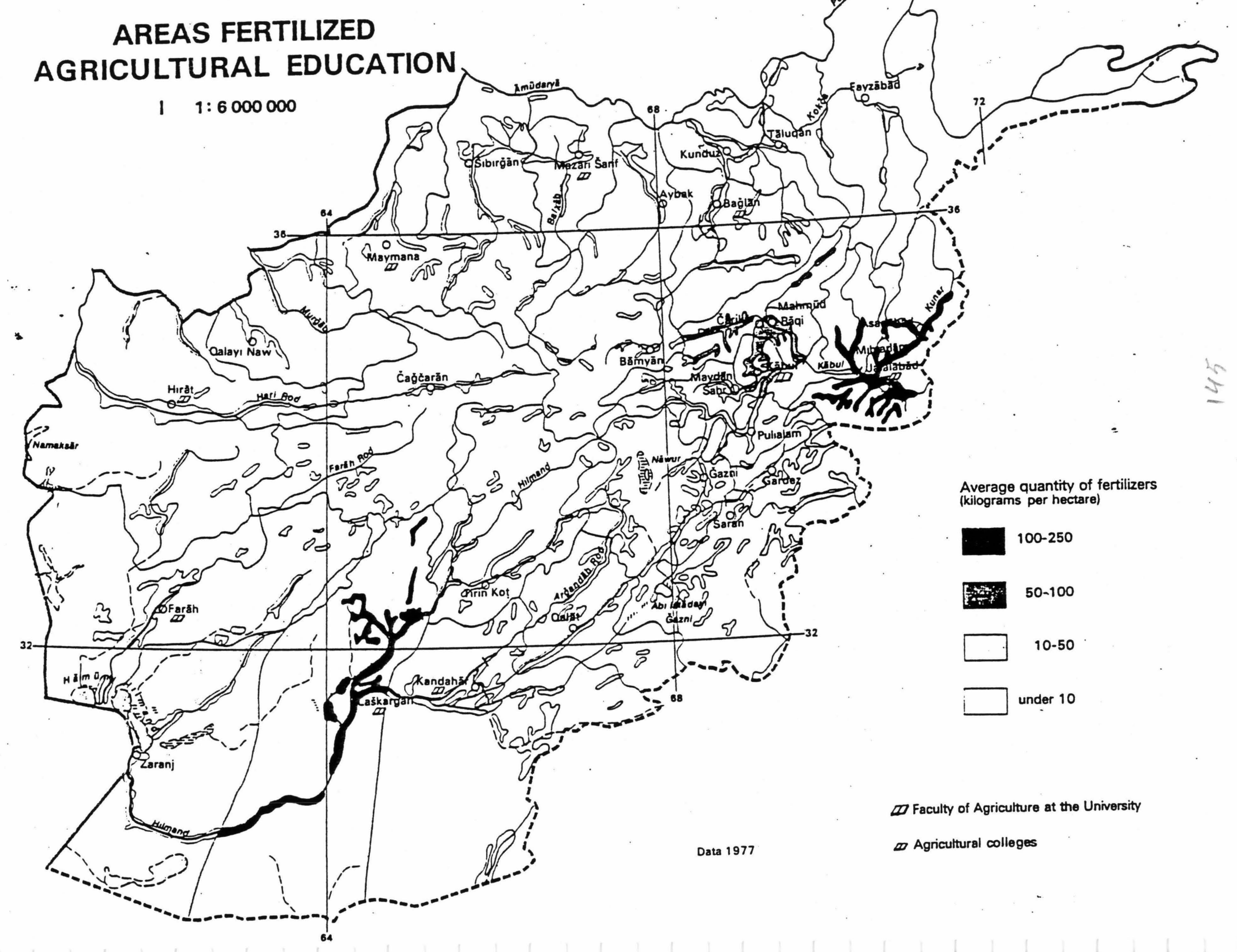




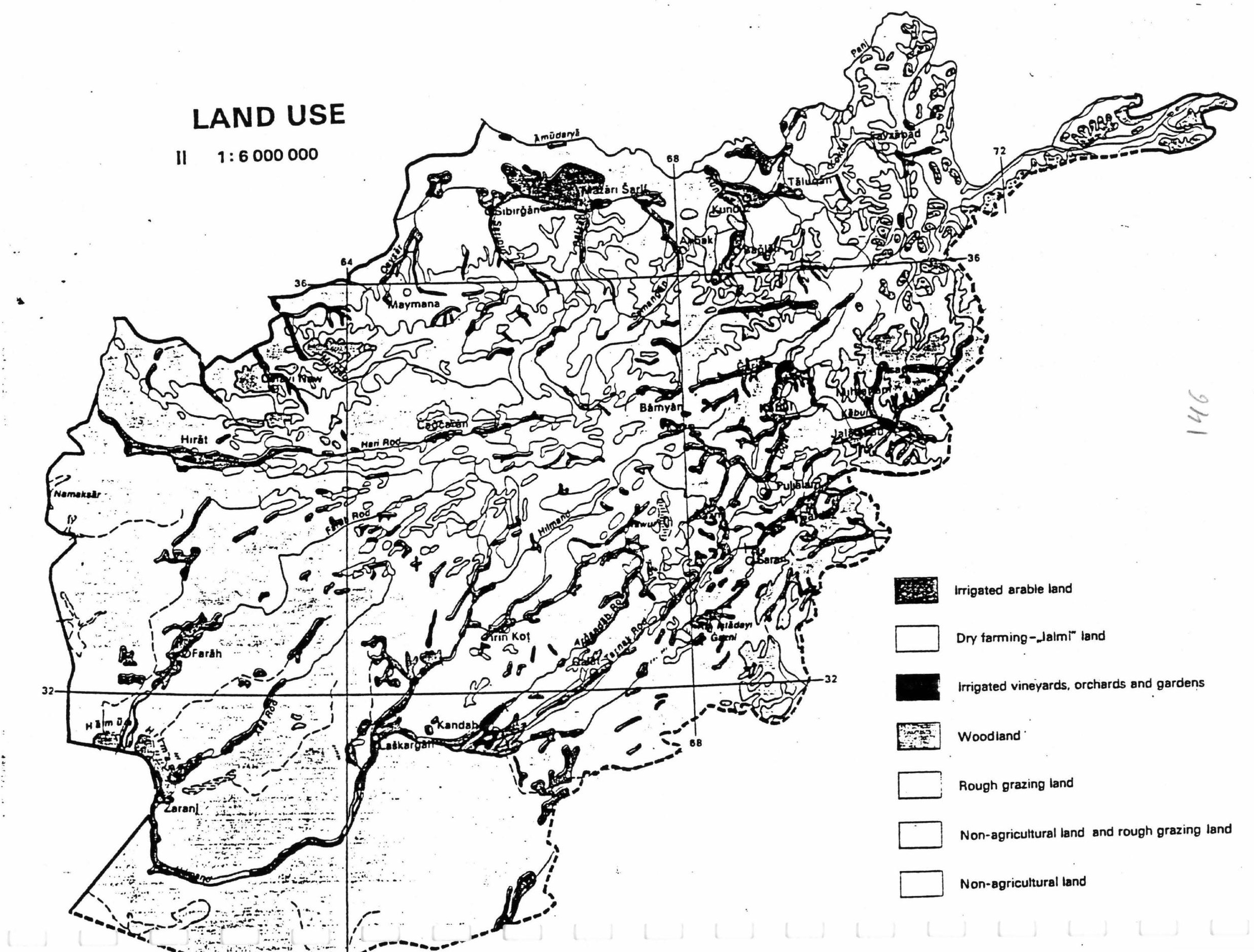




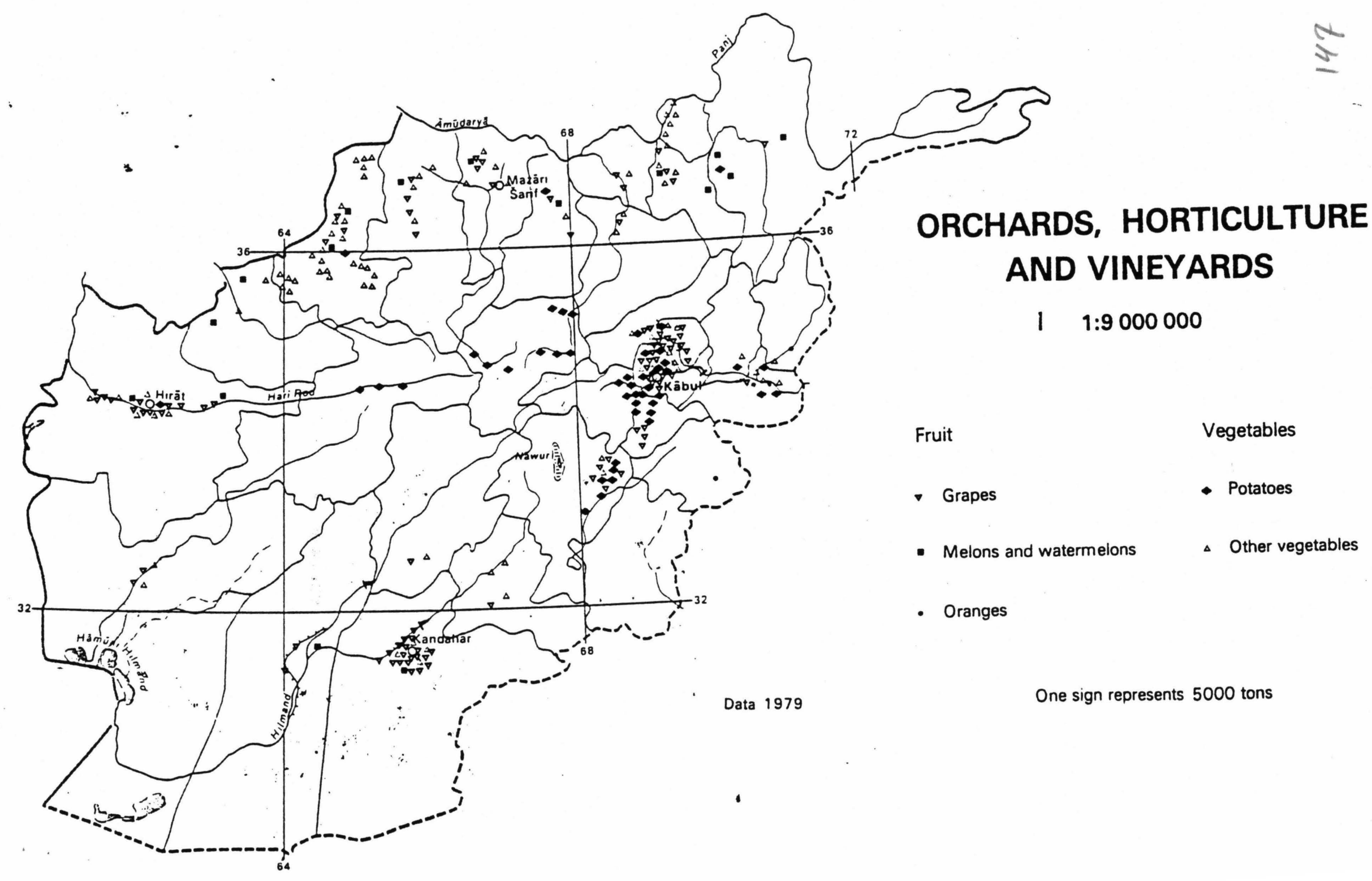




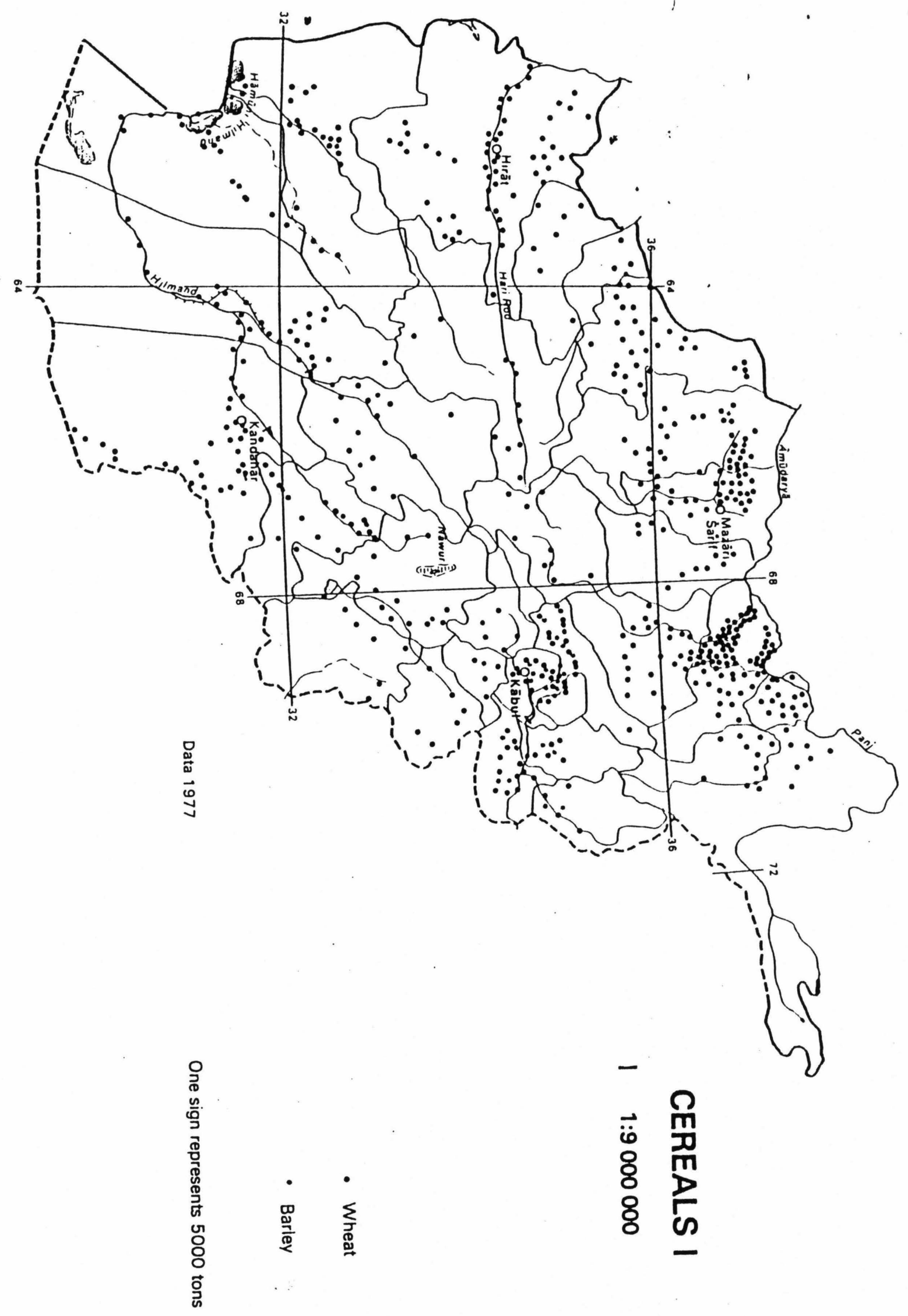




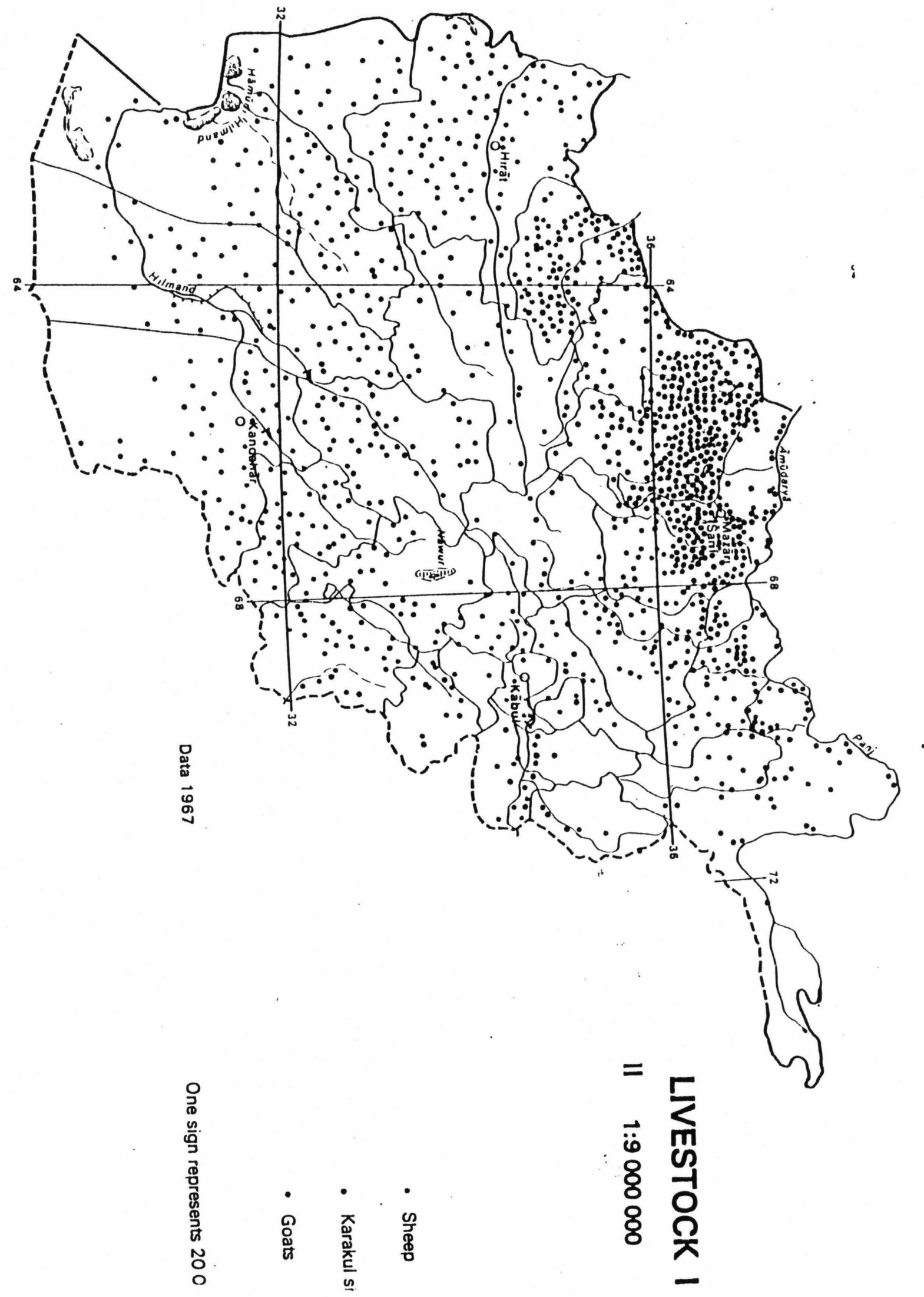




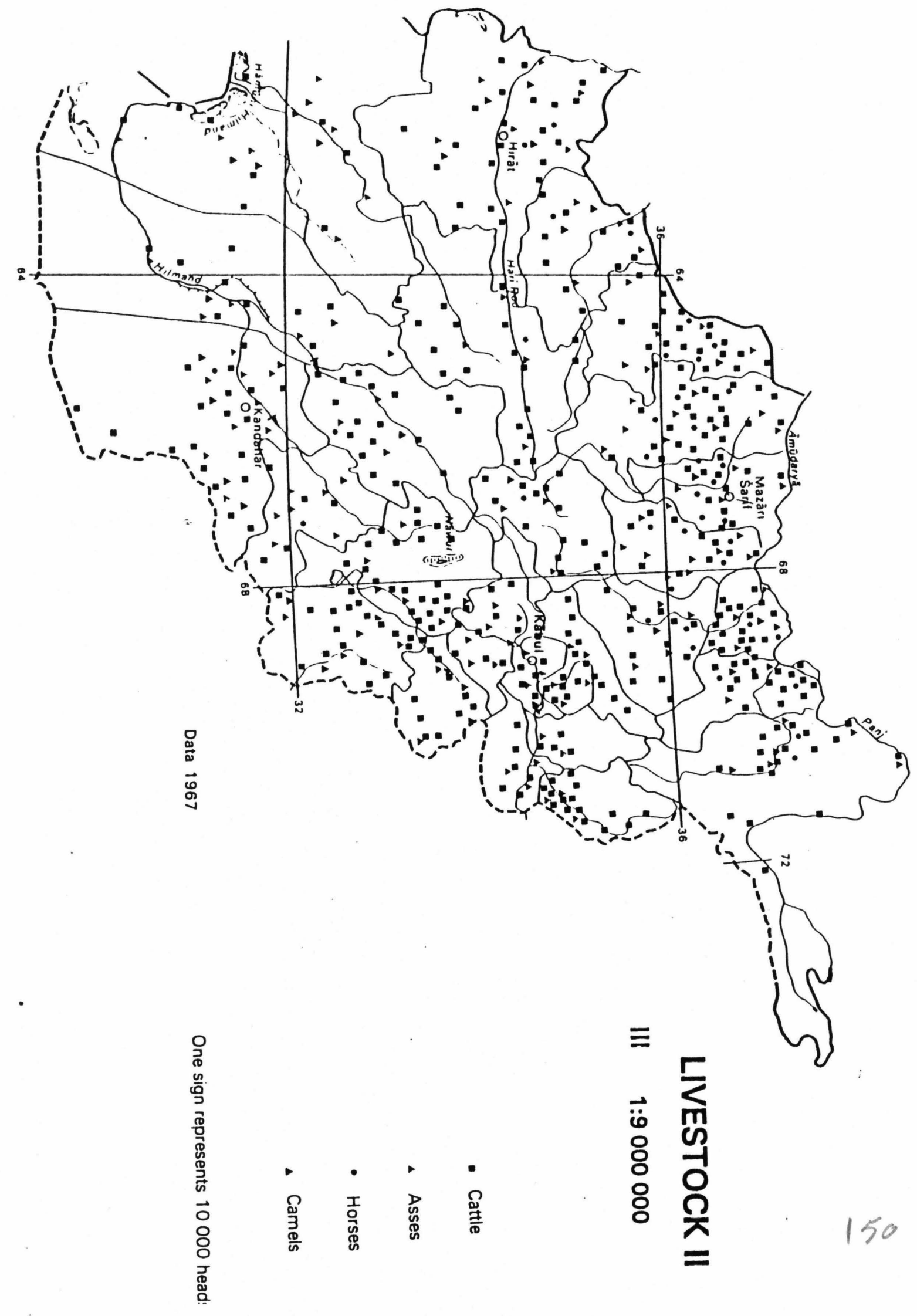

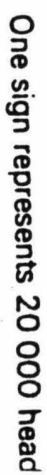




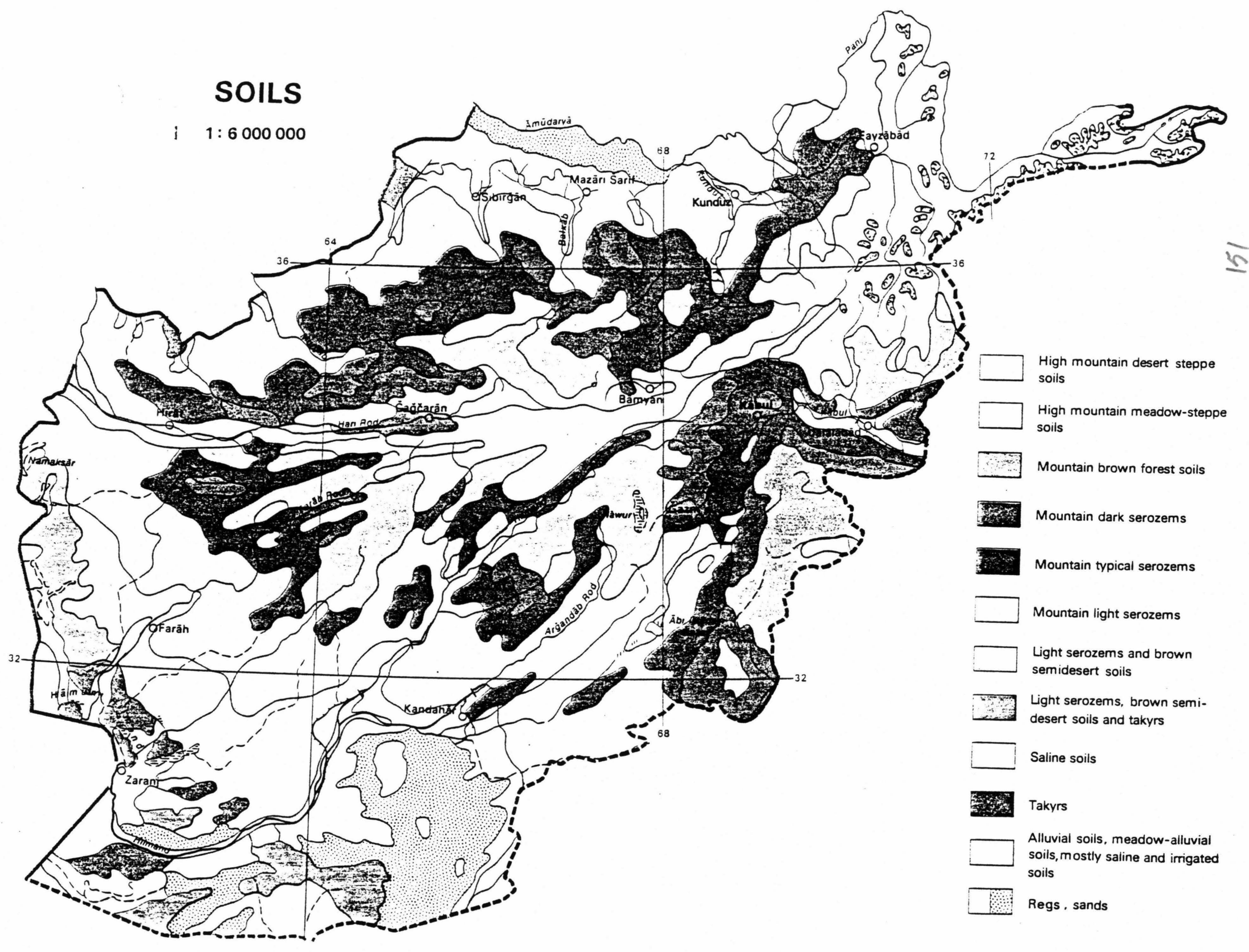




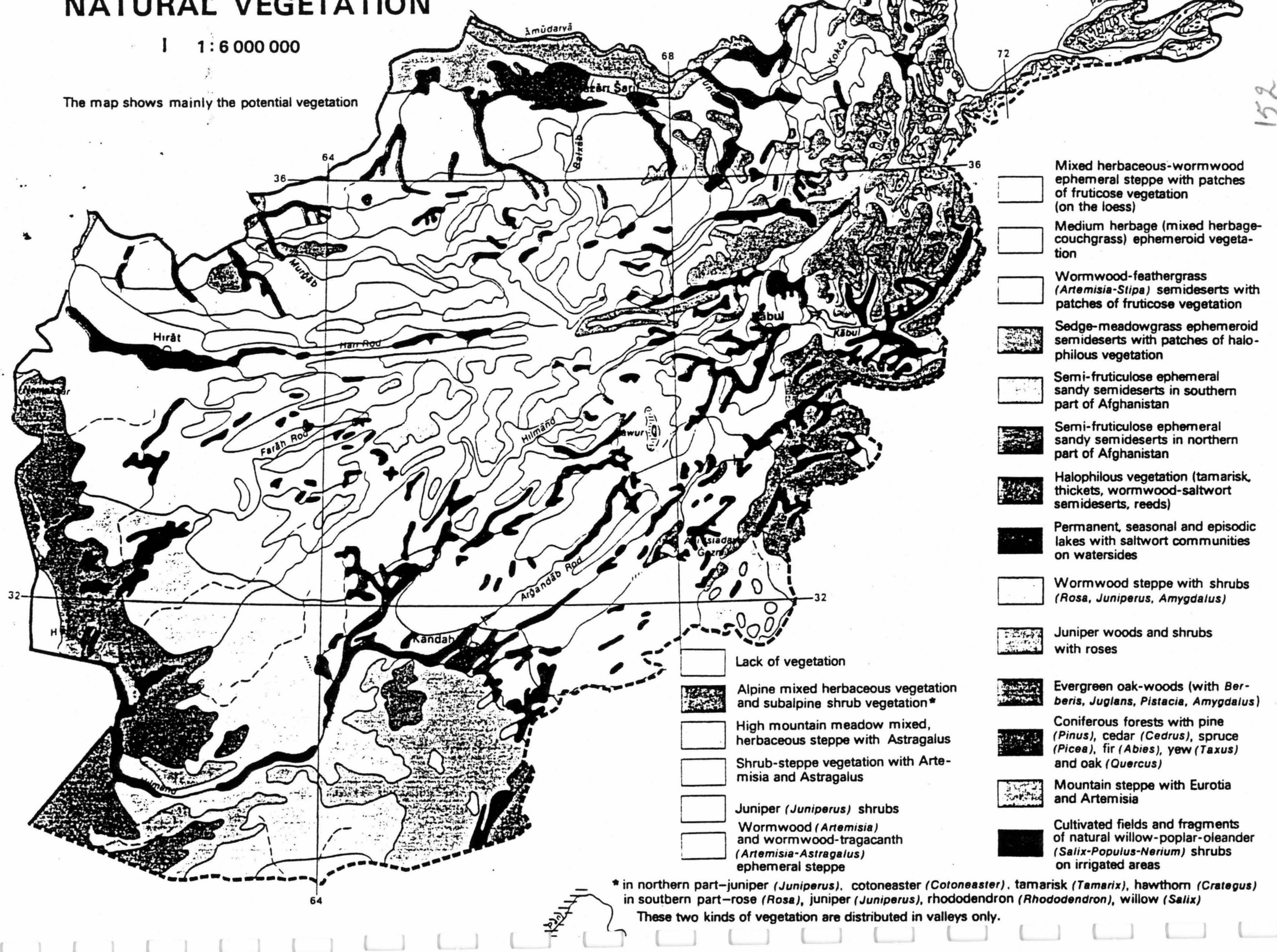




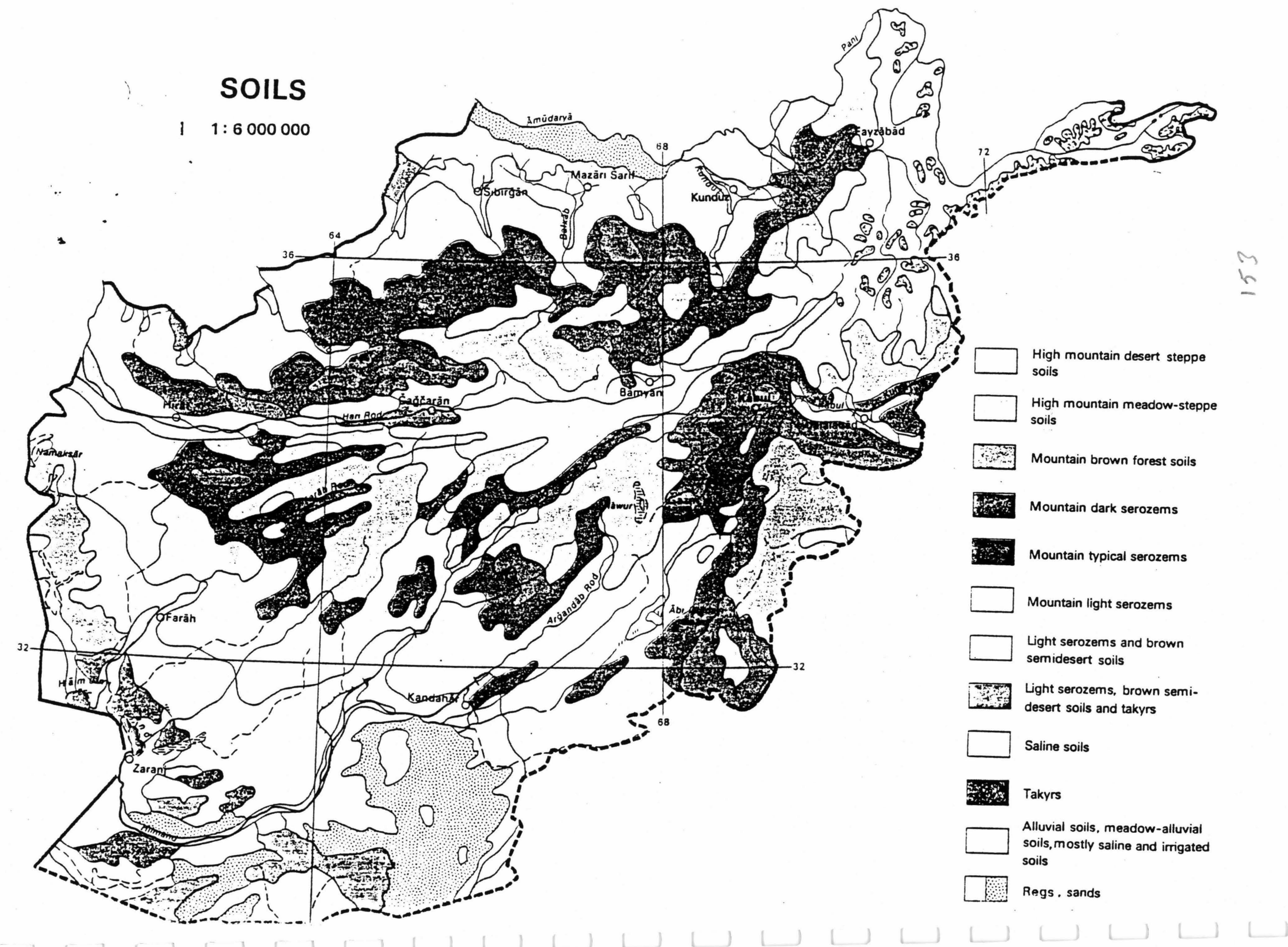




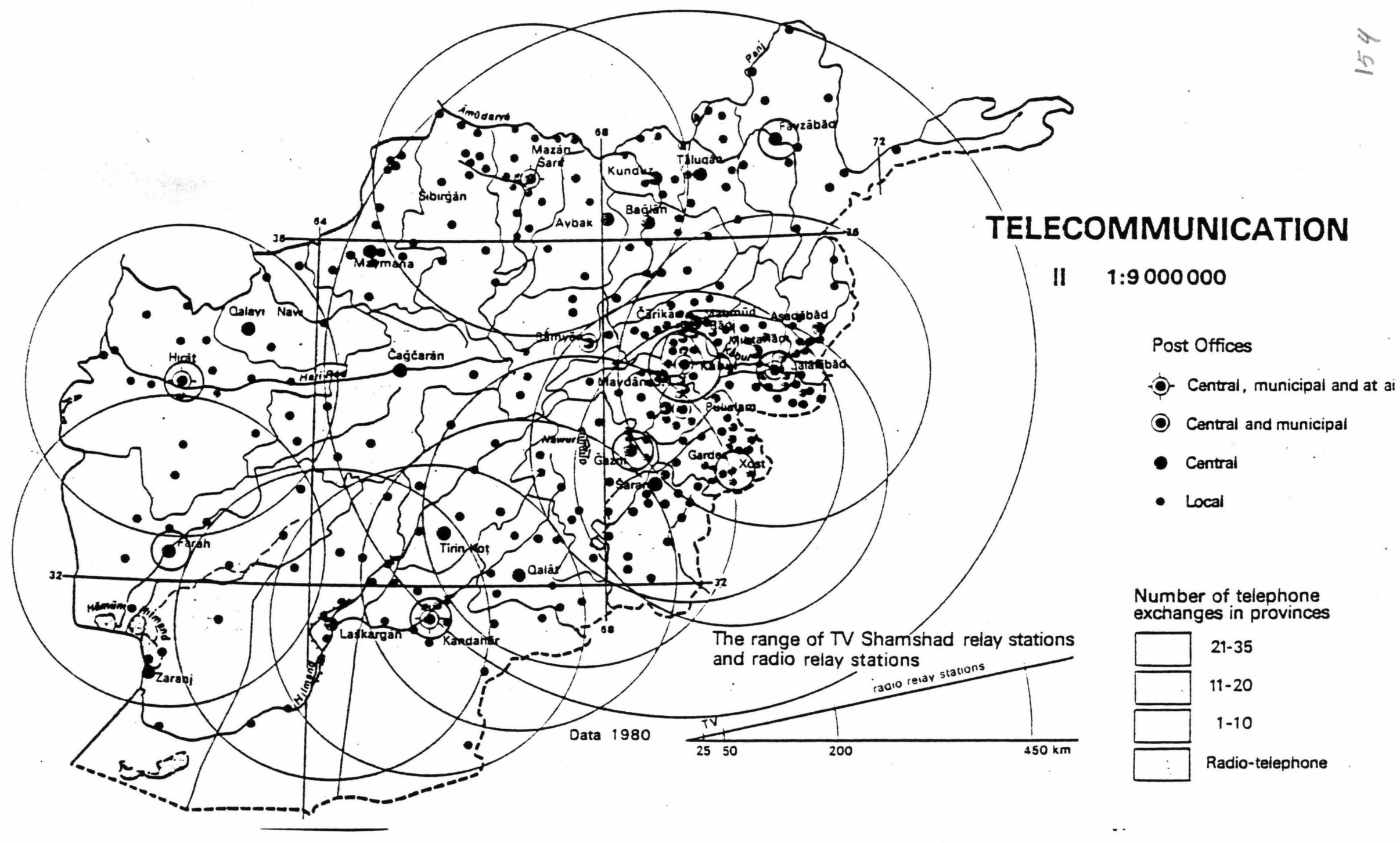




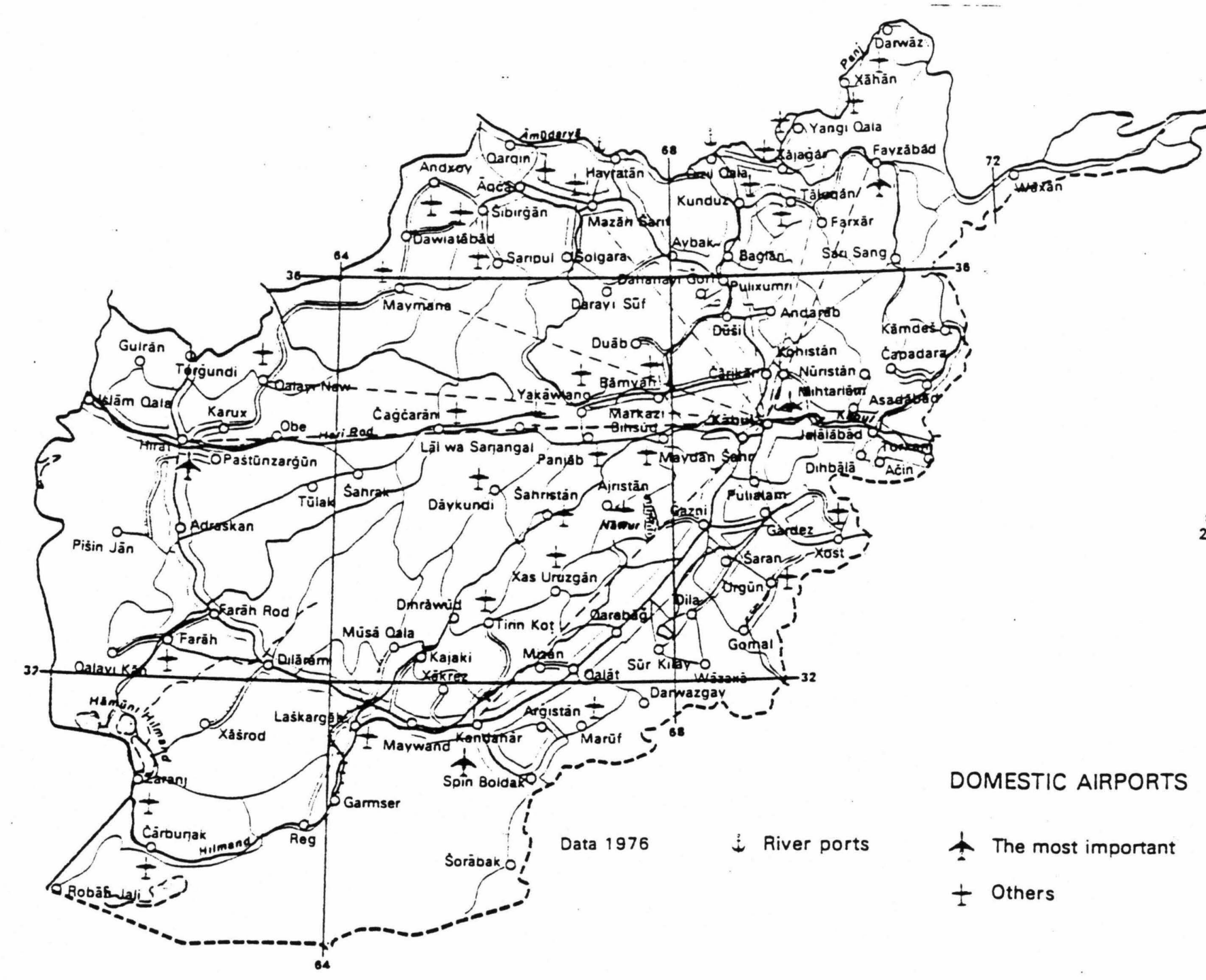

\section{TRANSPORT}

$1: 9000000$

ROAD TRAFFIC

Cars per day

$20 \quad 50 \quad 100250 \quad 500 \quad 1000$

Main roads

Connecting roads

Other roads

AIR TRAFFIC

Arrivals and departures (regular per year)

- - - Over 500

_. - Under 500 


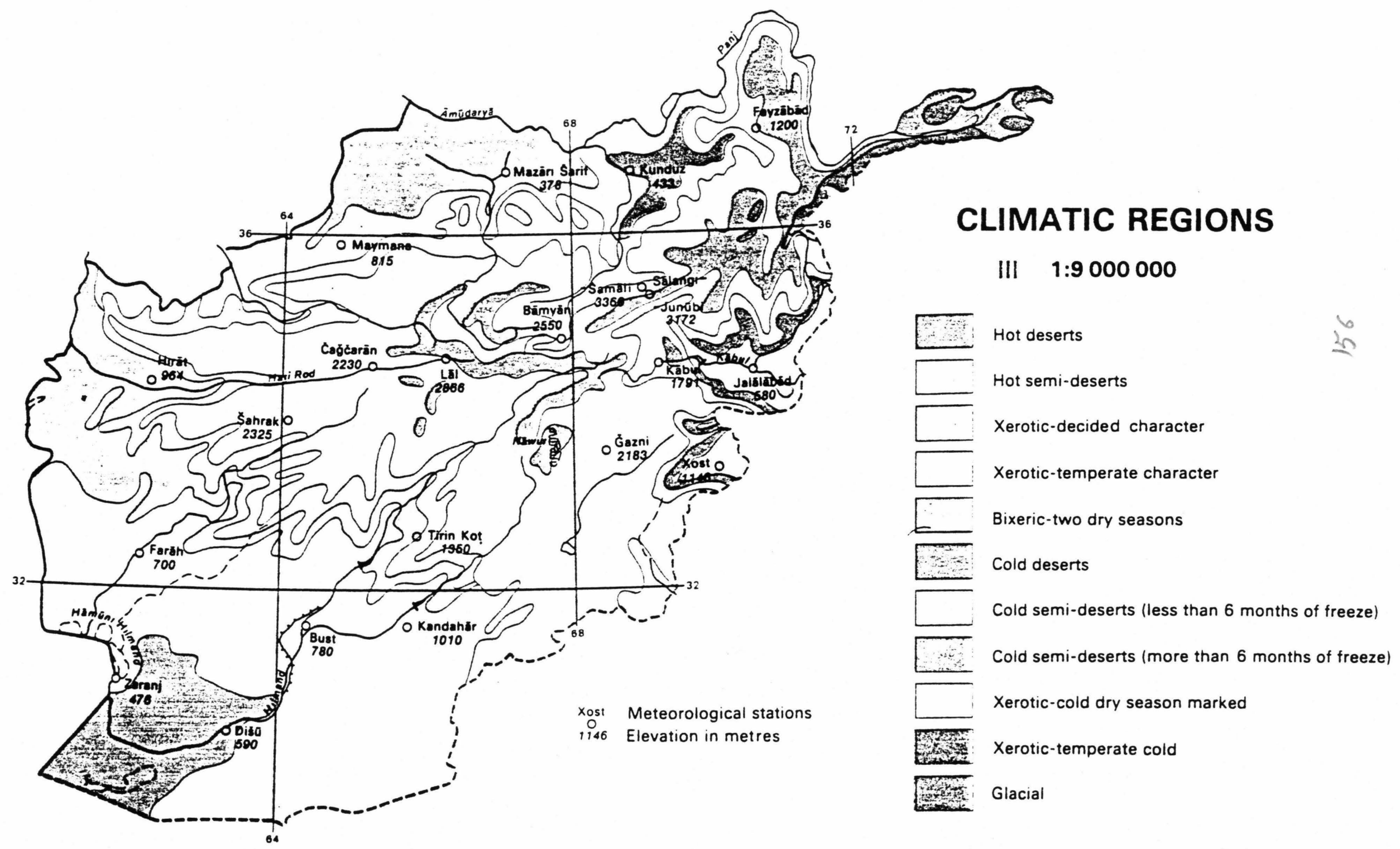




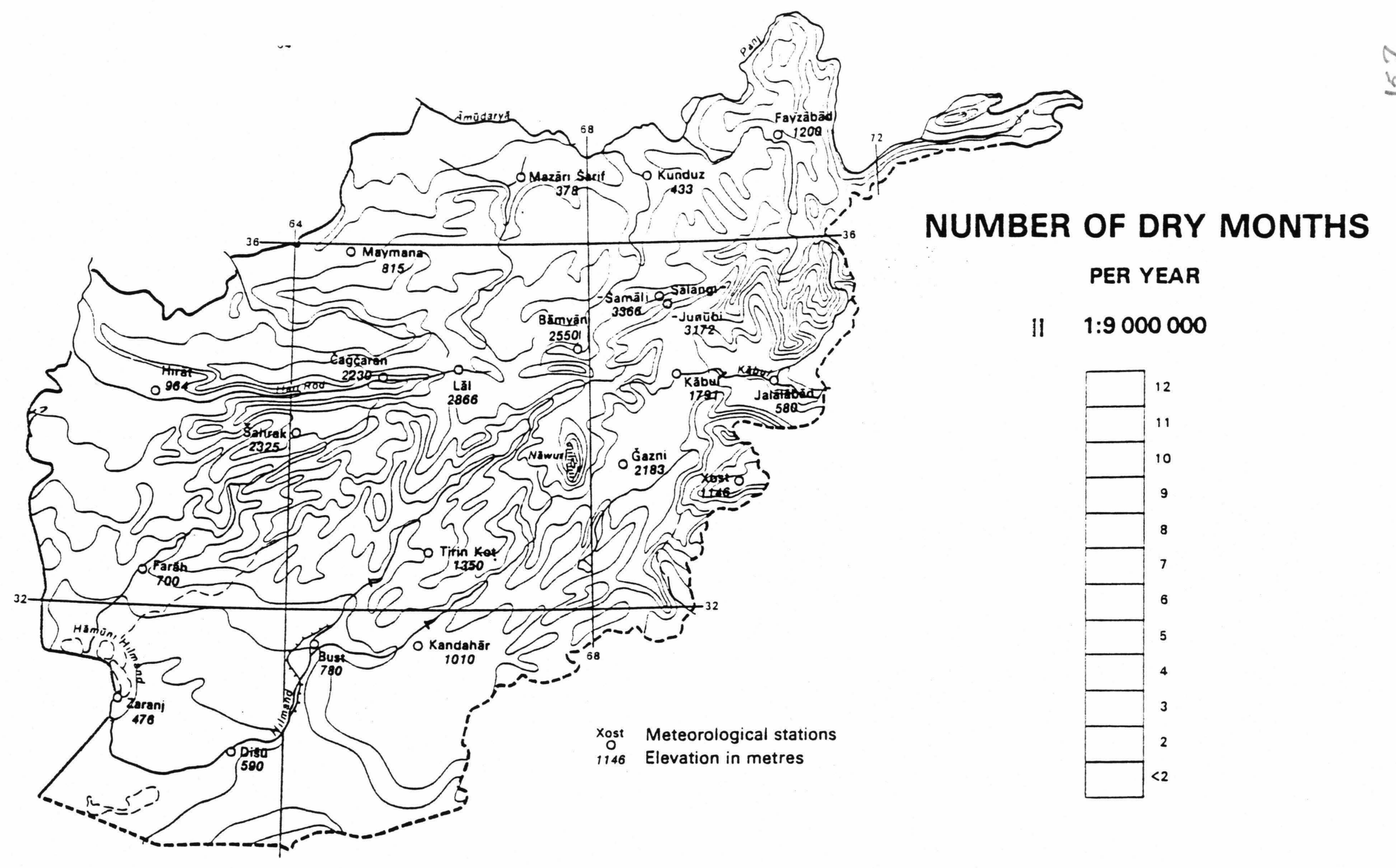




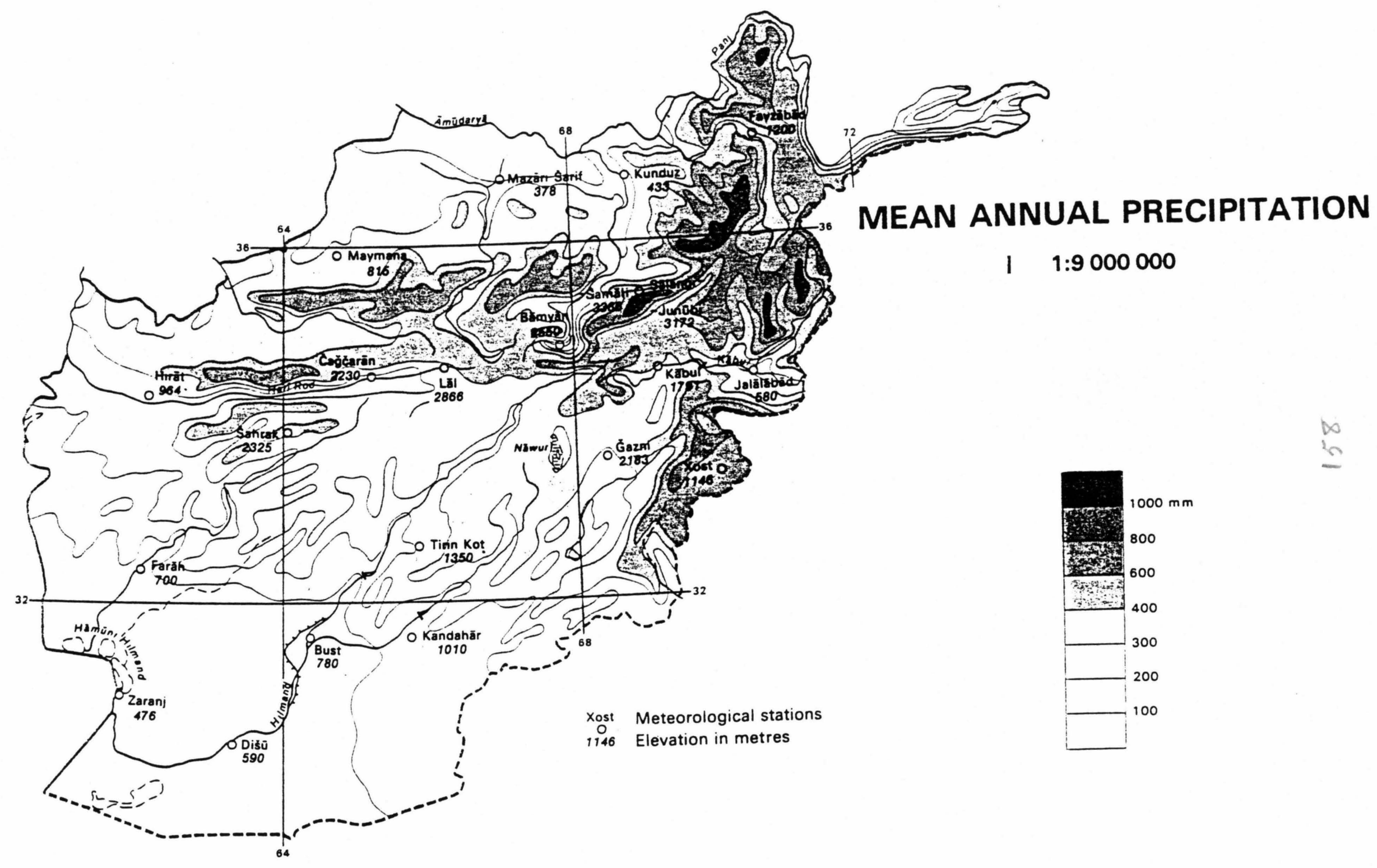




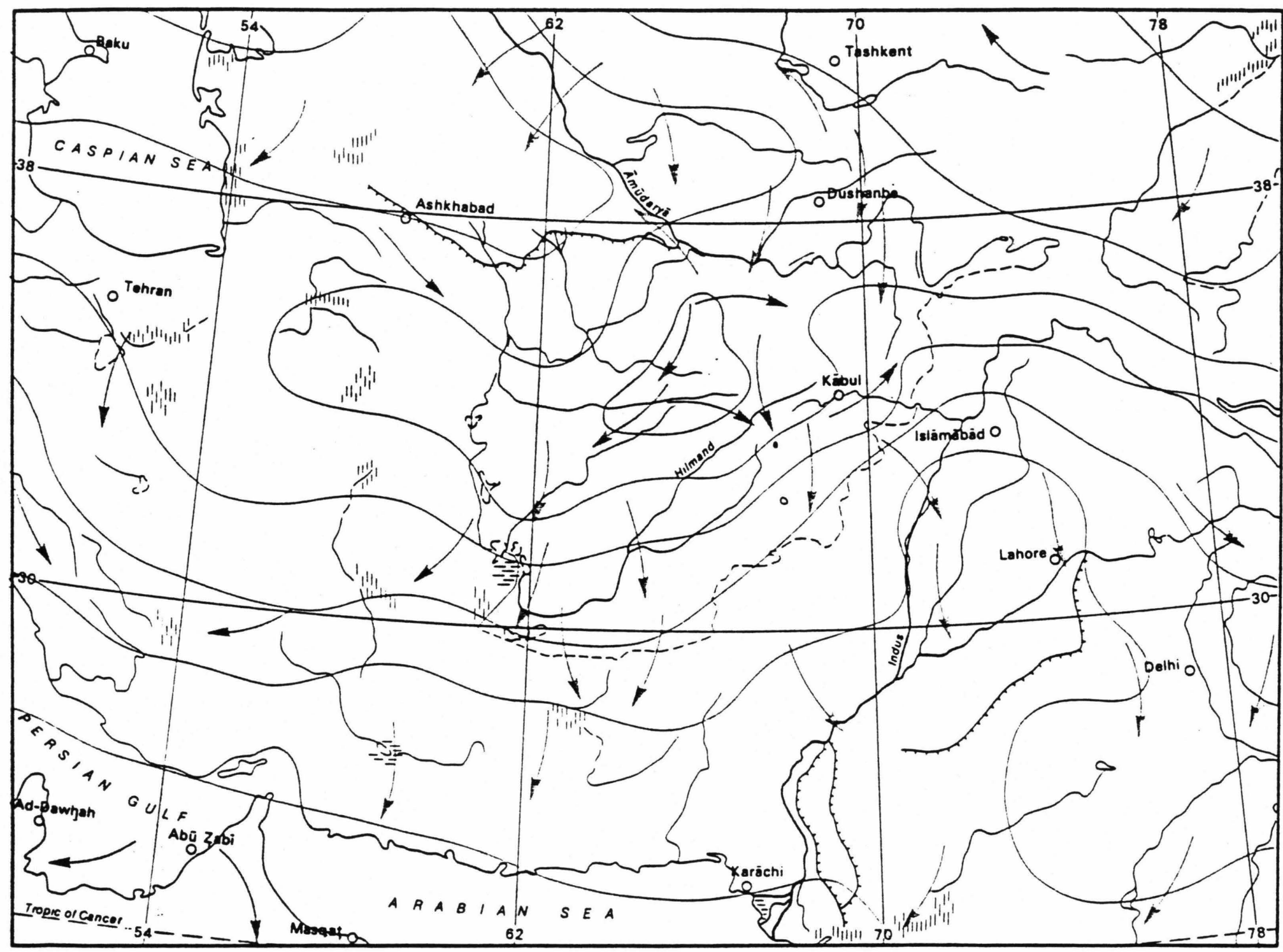

\section{WINTER PRESSURE} AND WINDS

\section{1:18000000}

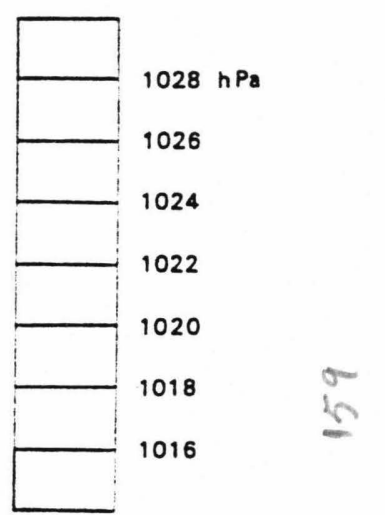




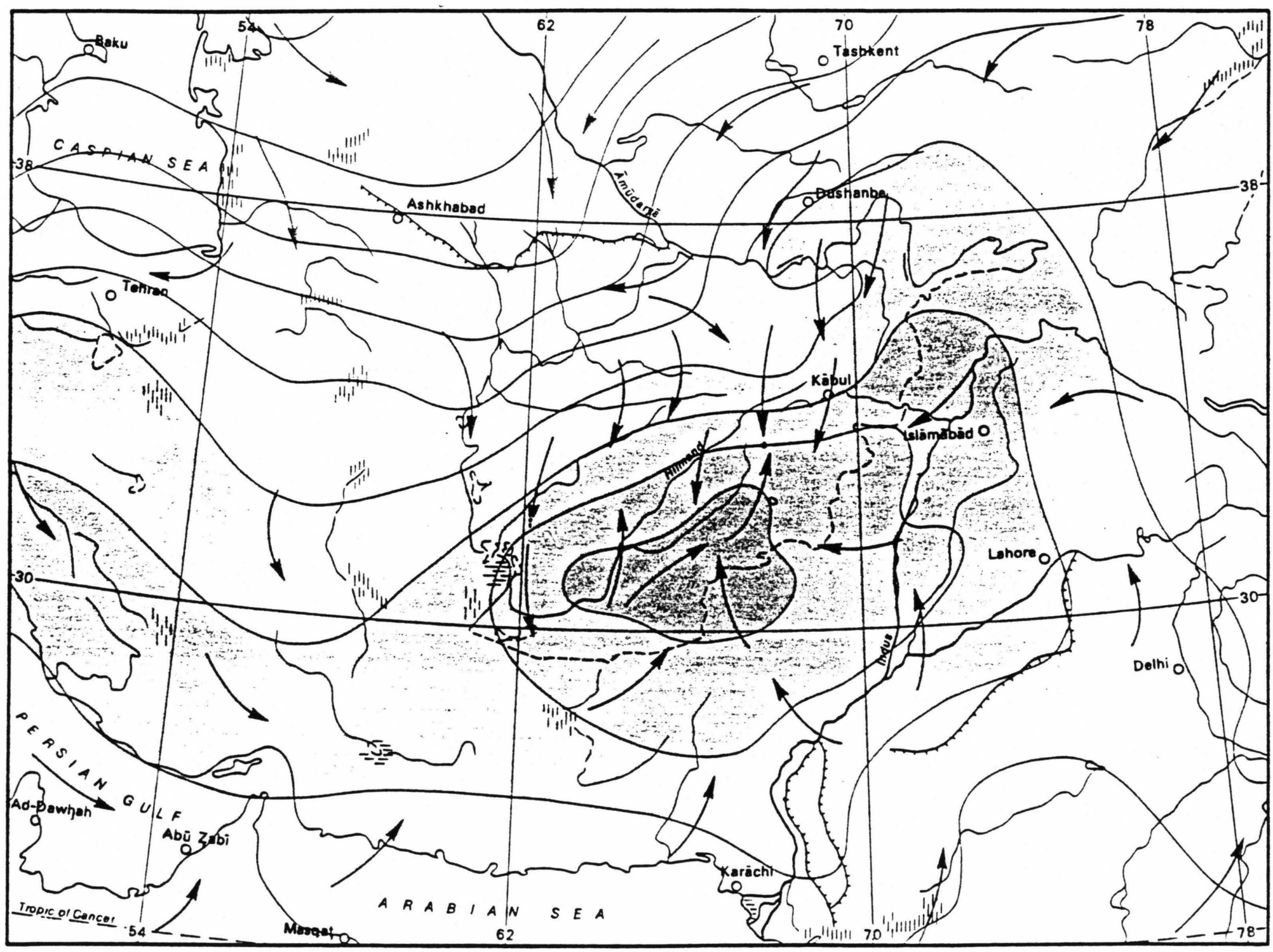

\section{SUMMER PRESSURE} AND WINDS

\section{1:18000000}

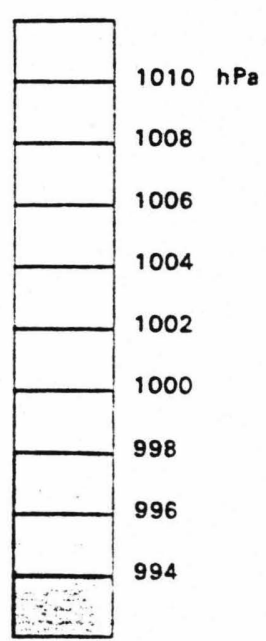

Dominant wind directions 


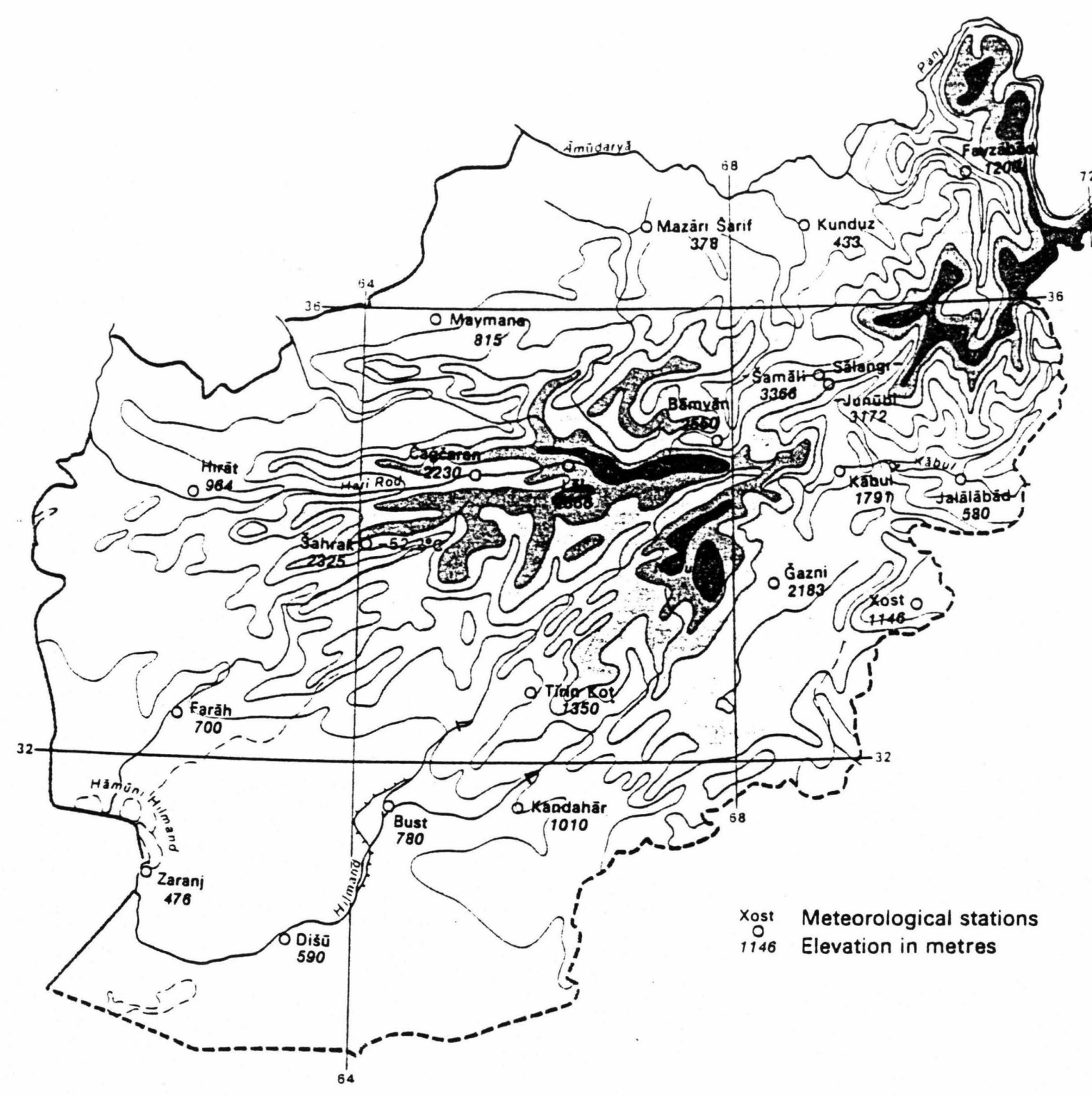

\section{TEMPERATURE OF THE COLDEST MONTH}

$1: 9000000$

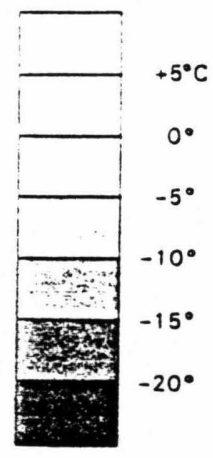

$-52.2^{\circ} \mathrm{C}$ Minimal temperature registered in Afghanistan 


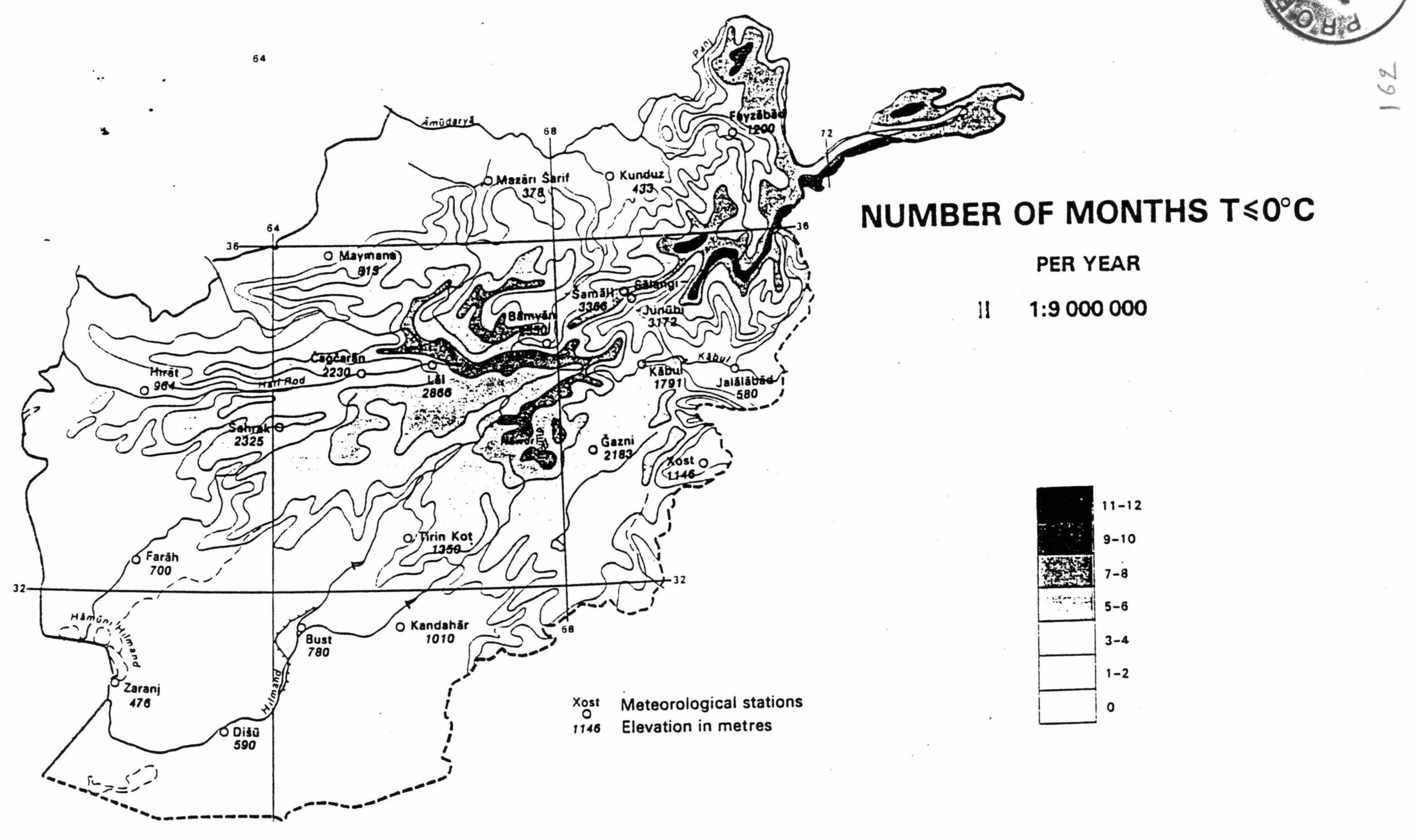




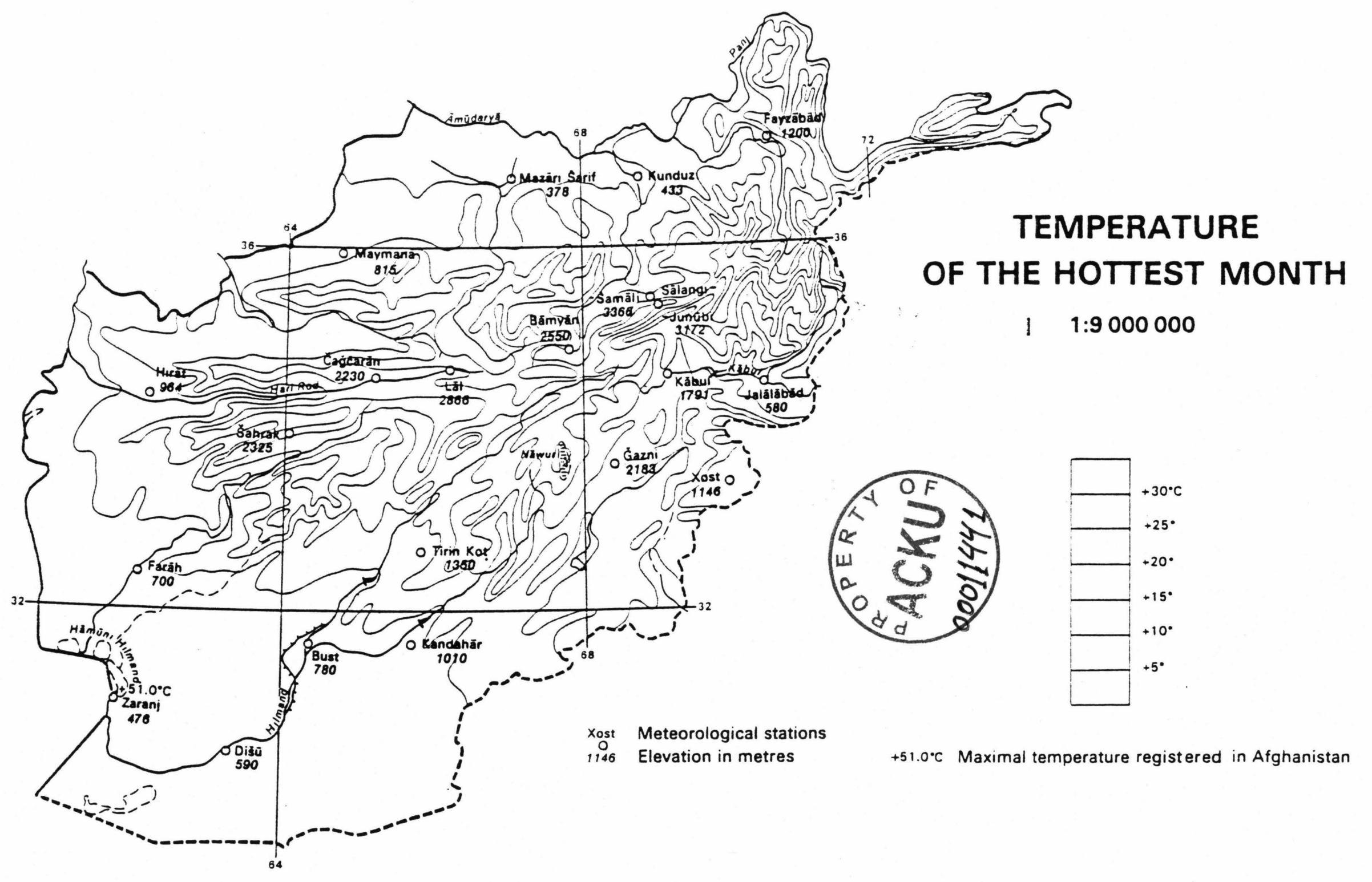




$$
\begin{gathered}
A R 16 \\
R \\
\hline 5731 \\
\hline U N A \\
\hline 11.441 \ldots
\end{gathered}
$$

